\title{
AN ORDER ESTIMATION BASED APPROACH TO IDENTIFY RESPONSE GENES FOR MICROARRAY TIME COURSE DATA
}

\author{
A Thesis \\ Presented to \\ The Faculty of Graduate Studies \\ of \\ The University of Guelph \\ by \\ ZHIHENG LU \\ In partial fulfilment of requirements \\ for the degree of \\ Doctor of Philosophy
}

September, 2008

(C) Zhiheng Lu, 2008 


$\begin{array}{ll}\begin{array}{l}\text { Library and } \\ \text { Archives Canada }\end{array} & \begin{array}{l}\text { Bibliothèque et } \\ \text { Archives Canada }\end{array} \\ \begin{array}{l}\text { Published Heritage } \\ \text { Branch }\end{array} & \begin{array}{l}\text { Direction du } \\ \text { Patrimoine de l'édition }\end{array} \\ \begin{array}{l}\text { 395 Wellington Street } \\ \text { Ottawa ON K1A 0N4 } \\ \text { Canada }\end{array} & \begin{array}{l}\text { 395, rue Wellington } \\ \text { Ottawa ON K1A 0N4 } \\ \text { Canada }\end{array}\end{array}$

Your file Votre référence ISBN: 978-0-494-47605-5 Our file Notre référence ISBN: 978-0-494-47605-5

NOTICE:

The author has granted a nonexclusive license allowing Library and Archives Canada to reproduce, publish, archive, preserve, conserve, communicate to the public by telecommunication or on the Internet, loan, distribute and sell theses worldwide, for commercial or noncommercial purposes, in microform, paper, electronic and/or any other formats.

The author retains copyright ownership and moral rights in this thesis. Neither the thesis nor substantial extracts from it may be printed or otherwise reproduced without the author's permission.
AVIS:

L'auteur a accordé une licence non exclusive permettant à la Bibliothèque et Archives Canada de reproduire, publier, archiver, sauvegarder, conserver, transmettre au public par télécommunication ou par l'Internet, prêter, distribuer et vendre des thèses partout dans le monde, à des fins commerciales ou autres, sur support microforme, papier, électronique et/ou autres formats.

L'auteur conserve la propriété du droit d'auteur et des droits moraux qui protège cette thèse. $\mathrm{Ni}$ la thèse ni des extraits substantiels de celle-ci ne doivent être imprimés ou autrement reproduits sans son autorisation.
In compliance with the Canadian Privacy Act some supporting forms may have been removed from this thesis.

While these forms may be included in the document page count, their removal does not represent any loss of content from the thesis.
Conformément à la loi canadienne sur la protection de la vie privée, quelques formulaires secondaires ont été enlevés de cette thèse.

Bien que ces formulaires aient inclus dans la pagination, il n'y aura aucun contenu manquant.

\section{Canada}


ABSTRACT

\section{AN ORDER ESTIMATION BASED APPROACH TO IDENTIFY RESPONSE GENES FOR MICROARRAY TIME COURSE DATA}

\section{Zhiheng Lu (Kevin)}

University of Guelph, 2008
Advisor:

Dr. B. Allen

Microarray time course experiments have been widely used to investigate temporal patterns of gene expression profiles. These expression profiles provide a unique opportunity to examine genome-wide signal processing and gene responses. A fundamental issue in microarray experimental design is that the treatment condition can only be controlled to the cell level rather than to the gene level. Given that some genes depend on other genes to detect changes in external conditions and that this kind of dependency is not fully deterministic and may vary across genes and treatment conditions, the expression of each gene is potentially affected by two confounding effects: the treatment effect and the gene context effect arising from the regulatory interaction structure among genes. This gene context effect is hard to isolate. Neither can it be simply ignored. Instead, this gene context information which is different under different treatment conditions is of primary biological interest and thus demands attention of statistical analysis. 
We introduce an approach which provides a way to deal with the confounding effects and takes into account the uncontrollable gene context effect. Our method is developed to estimate the number of hidden states which is also referred to as the order of a hidden Markov model (HMM) for each gene. The observed gene expressions are modeled by gamma distributions determined by the corresponding hidden state at each time point. Those genes showing evidence for more than one hidden state can be categorized as the signaling genes, or in a wider sense, as the response genes which are coordinated by a cell system in reaction to a specific external condition. These response genes can be used in the comparison of different treatment conditions, to investigate the gene context effect under different treatments. Our method also provides flexibility in adjusting type I error rates to find response genes at different response intensity levels. Both simulated data and real microarray time course data are analyzed to demonstrate our method. 


\section{Acknowledgements}

I sincerely thank my advisor Dr. Brian Allen for his insightful guidance, support and help during my study. I would like to thank my other advisory committee members, Dr. T. Desmond, Dr. R. Lu, Dr. G. Darlington and Dr. J. Horrocks for their treasured advice and help. Dr. A. Canty deserves special thanks for very extensive and constructive suggestions which have greatly improved this thesis. 


\section{Table of Contents}

List of Tables ............................................................. iv

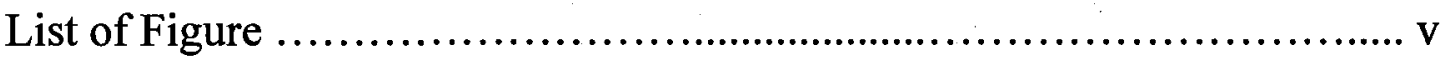

1 Introduction 1

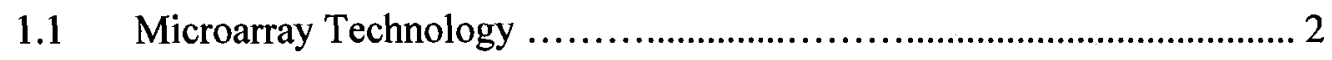

$1.2 \quad$ Microarray Time Series Experiments ............................................... 7

1.3 Microarray Data Pre-processing ................................................ 22

2 General Model 27

3 Empirical Bayes Estimation of Shape Parameter 34

4 Order Estimation $\quad 42$

4.1 Literature Review of Order Estimation for HMMs and Finite Mixture

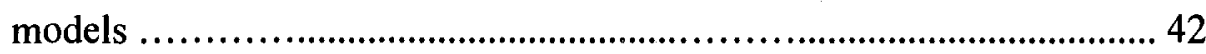

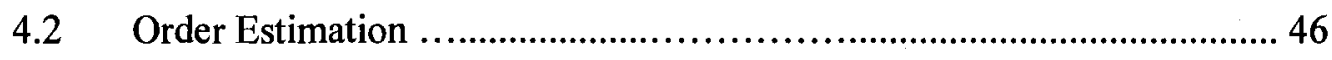

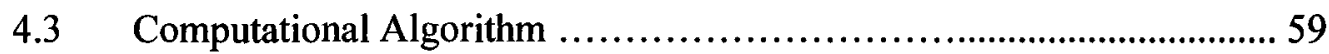

4.4 Choices of the Threshold Parameters .............................................. 64

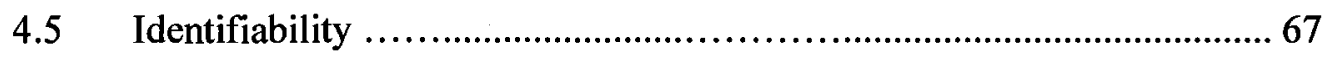

$\begin{array}{ll}5 \text { Simulation Study } & 70\end{array}$

6 Real Data Analysis $\quad 89$

7 Conclusions and Further Discussion $\quad 99$

$\begin{array}{ll}\text { References } & 104\end{array}$ 


\section{Table of Contents}

$\begin{array}{ll}\text { Appendix A } & 116\end{array}$

Appendix A.1, Response gene set for GDS1428 control group ......................... 116

Appendix A.2, Response gene set for GDS1428 treatment group ..................... 123

Appendix A.3, The common response gene set for GDS1428 treatment group and control group ....................................................................... 131

Appendix A.4, The response gene set only for GDS1428 control group .......... 136

Appendix A.5, The response gene set only for GDS1428 treatment group ...... 139

Appendix A.6, The common gene set of gene set A and B ......................... 144

Appendix A.7, The genes in gene set $A$ but not in gene set $B$.................... 145

Appendix A.8, The genes not in gene set $\mathrm{A}$ but in gene set B .................... 148

Appendix A.9, The common gene set of gene set $A$ and $C$...................... 155

Appendix A.10, The genes in gene set $A$ but not in gene set $C$......................... 156

Appendix A.11, The genes not in gene set $A$ but in gene set $C$.................... 159

Appendix B, Computation codes in R 163 


\section{List of Tables}

Table 5.1, The four situations of order estimation ........................................................... 72

Table 5.2, Type I error rate at three separation thresholds for various single gamma

distributions

Table 5.3, The specifications for the simulation of mixtures of two gamma distributions

Table 5.4, Simulation evaluations for the mixture models with 2 components .............. 83

Table 5.5, Simulation evaluations for the mixture models with 3 components ............... 84

Table 5.6, Simulation evaluations for HMMs with 2 hidden states ....................... 87

Table 6.1, The number of response gene for each treatment condition of GDS1428 ......93

Table 6.2 , The comparison of gene lists between the two studies ...................... 98 


\section{List of Figures}

Figure 1.1, A schematic of the role of RNA in gene expression and protein production 3

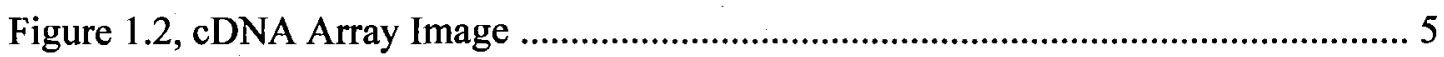

Figure 1.3, GeneChip Affymetrix Array Image ......................................................... 5

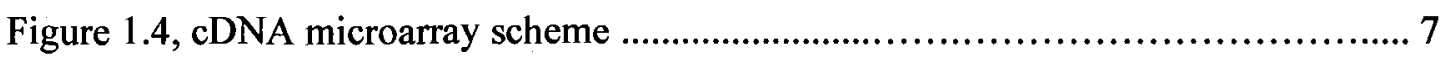

Figure 1.5, Gene expression profiles for 8 genes at time points $0,1.5,3,6,9,12$ and 24

hours

Figure 1.6, Microarray time course experiment under one specific treatment

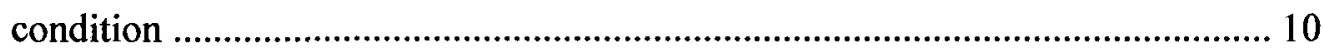

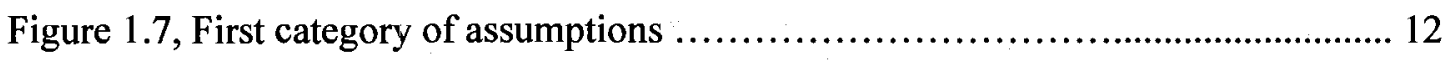

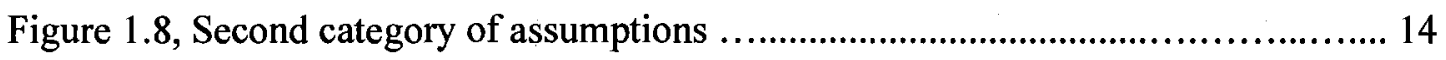

Figure 4.2.1, A gamma mixture with three component distributions .......................... 53

Figure 6.1, Plot of CV vs. probe (gene) index for treatment and control data for

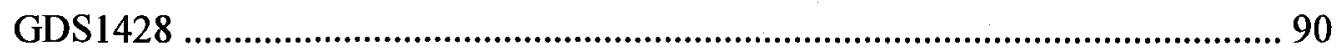

Figure 6.2, Plot of shape parameter vs. probe (gene) index for treatment and control data

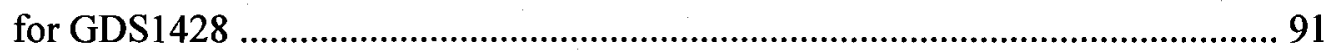




\section{Chapter 1}

\section{INTRODUCTION}

Microarray technology makes it possible for researchers to simultaneously assess the expression of thousands of genes (Schena et al., 1995; Lockhart et al., 1996; Richmond et al., 1997; Eisen et al., 1998). A huge amount of data that represents the expression patterns of potentially all of the genes in a cell under various treatments are generated by the scientific community and becomes available for statistical analysis. See Craig et al. (2003) for a more detailed review. In this chapter, we will first briefly introduce microarray technology in section 1.1 . We then provide a description of microarray time course experiments in section 1.2 and explain the motivation and the goal of our order estimation analysis. In section 1.3 , we briefly introduce microarray data pre-processing. 


\subsection{Microarray Technology}

According to the central dogma of molecular biology (Snustad and Simmons, 2003), many key biological functions of cells are performed by proteins. The production of proteins is controlled by genes, which are coded in DNA sequences and passed along generations of cells by the DNA replication process. Protein production from genes involves two stages, known as transcription and translation, as shown in Figure 1.1. A single strand of messenger RNA or mRNA is first copied from the DNA sequence of the coding gene. After transcription, mRNA is used as a template to assemble a chain of amino acids to produce the protein during the translation stage. Since most of the functions of cellular biological processes are related to the changes of mRNA levels for some genes, systematic investigation of mRNA abundance on a genome-wide scale is critical in understanding cell systems. Microarray technology makes it possible to examine gene expression for any number of genes simultaneously, and hence for the first time in history, the comprehensive measurement of a large system as complex as a cell becomes available. The understanding of microarray data, however, turns out to be much more challenging than expected. 


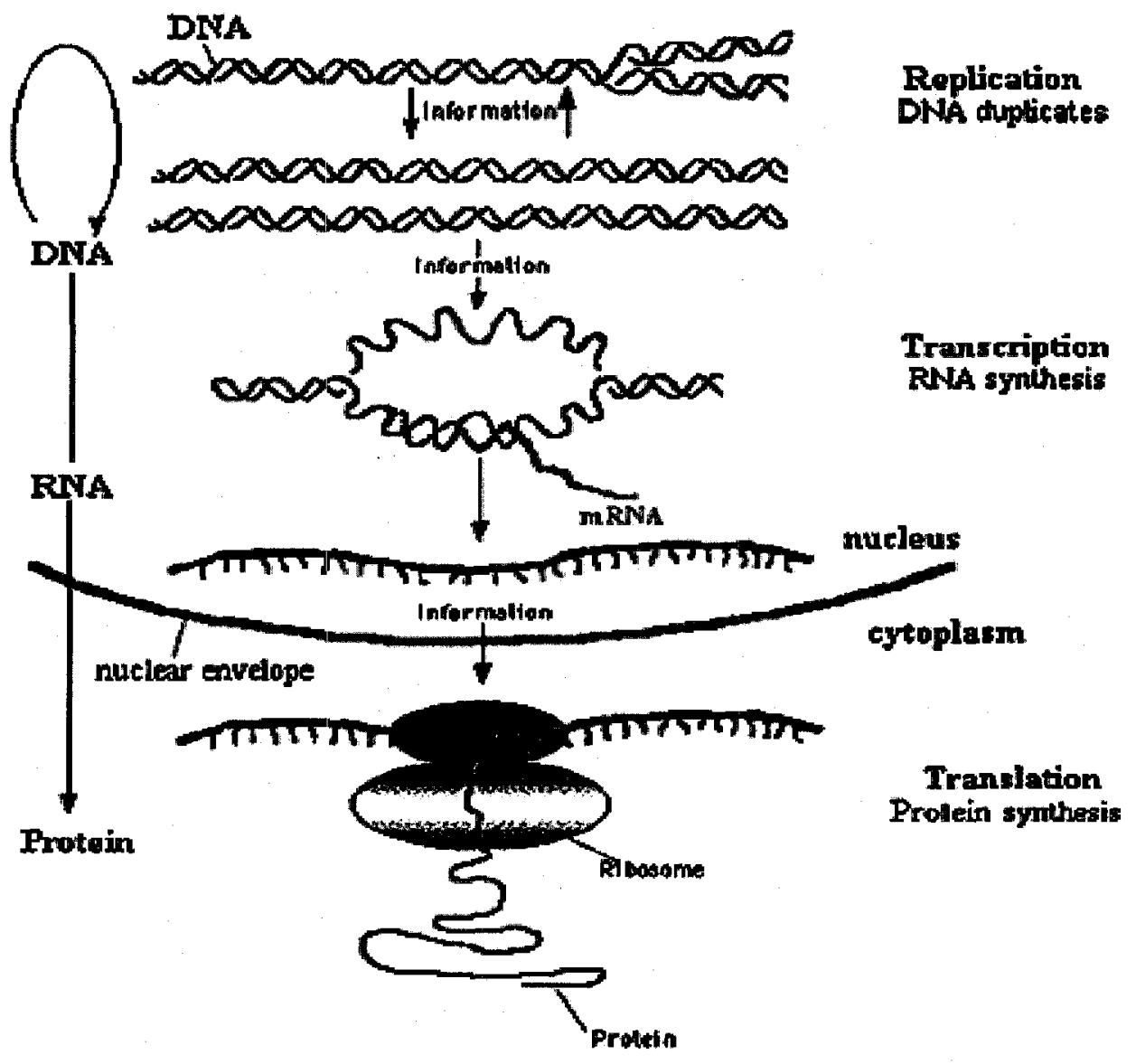

The Central Dogma of Molecular Biology

Figure 1.1, A schematic of the role of RNA in gene expression and protein production.

Graphics from http://www.accessexcellence.org.

There are two different microarray technologies, spotted arrays (also referred to as cDNA arrays) (Schena et al., 1995) and oligonucleotide arrays (Lockhart et al., 1996). The images for these two types of microarrays are shown in Figure 1.2 and Figure 1.3 respectively. To quantify gene expression levels in a cell or organism, microarray technology utilizes the natural affinity of single stranded DNA to bind with its 
complementary sequence (either DNA or RNA sequence). There are four bases in a DNA sequence: A (adenine), $\mathbb{T}$ (thymine), G (guanine) and C (cytosine). In an RNA sequence, the base $\mathrm{T}$ is replaced by $\mathrm{U}$ (uracil). According to molecular biology, base A pairs with base $T$ (or base $U$ in the case of RNA sequences) and base $C$ pairs with base $G$. A large number of known DNA sequences are first attached to the surface of an array chip or slide. These sequences are referred to as probes. The probes from the same sequence are attached to a small region referred to as a spot on a microarray slide surface. Then the genetic material (i.e. mRNA) is extracted from a cell or an organism. In some situations, mRNAs are further reverse-transcribed into cDNA. These sequences are referred to as targets. Then targets from a specific biological sample are labeled with a fluorescent dye and allowed to be hybridized to the probes. A laser scanner is used to measure the color intensity of the dye fluorescence for each spot on a slide. Higher fluorescence intensity indicates larger amounts of hybridized targets, which further indicates a higher expression level for the corresponding gene. The scanned slide images are shown as in Figure 1.2 and Figure 1.3. 

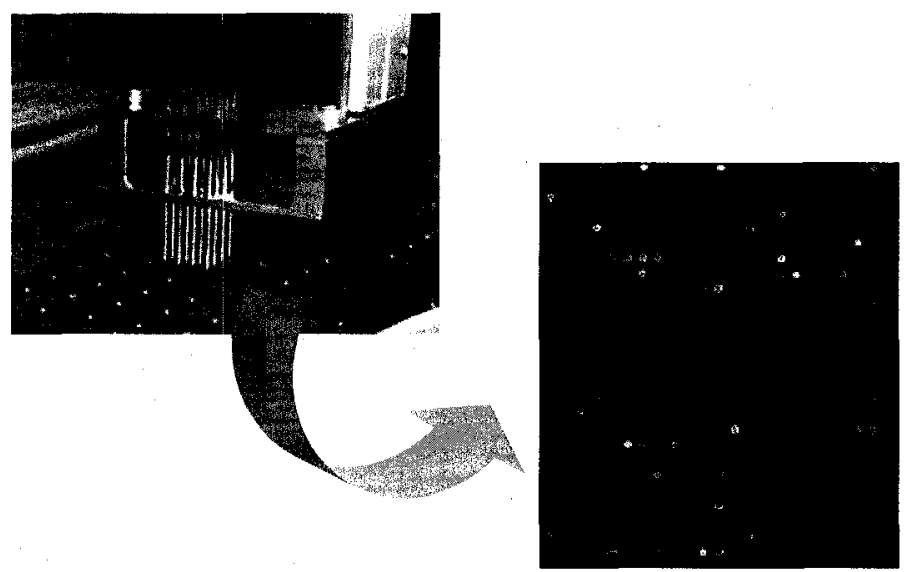

Figure 1.2, cDNA Array Image. Graphics adapted from presentation "Statistical Issues in the Design of Microarray Experiment", Jean Yee Hwa Yang, University of California, San Francisco, http://www.biostat.ucsf.edu/jean/.

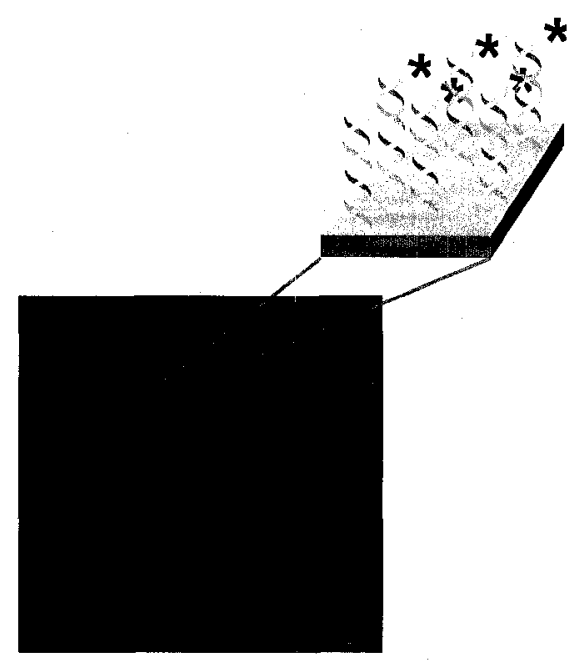

Figure 1.3, GeneChip Affymetrix Array Image. Graphics adapted from presentation "Statistical Issues in the Design of Microarray Experiment", Jean Yee Hwa Yang, University of California, San Francisco, http://www.biostat.ucsf.edu/jean/. 
Targets are labeled slightly differently between spotted arrays and oligonucleotide arrays. For spotted arrays, samples from two different treatment conditions are prepared. The targets are labeled with two fluorescent dyes: Cy3 (green) or Cy5 (red). Then these targets are mixed together and hybridized competitively to the probes. The images scanned for these spotted arrays have potentially two colors for each spot as shown in Figure 1.2. The major steps in a microarray experiment are illustrated in Figure 1.4. The oligonucleotide array is prepared in a similar way to cDNA arrays.

Oligonucleotide arrays use only one color for each spot. The targets are labeled with a single color and hybridized to the probes. For the most widely used Affymetrix GeneChip arrays, normally 11-20 pairs of probes, of length 25 bases, are attached to a GeneChip for each known sequence. In each pair, one is the probe with known DNA sequence, known as perfect match (PM). The other is a sequence with the middle base (the 13th base of the 25 bases) changed, known as mismatch (MM). The MMs are used to control for experimental variation and to measure nonspecific binding of targets from other genes. Since the data analyzed in chapter 6 are from Affymetrix oligonucleotide microarrays, our subsequent discussion will focus on this type of microarray, and we further focus our discussion on time course microarrays performed for the purpose to investigate the regulatory relationship among genes. 


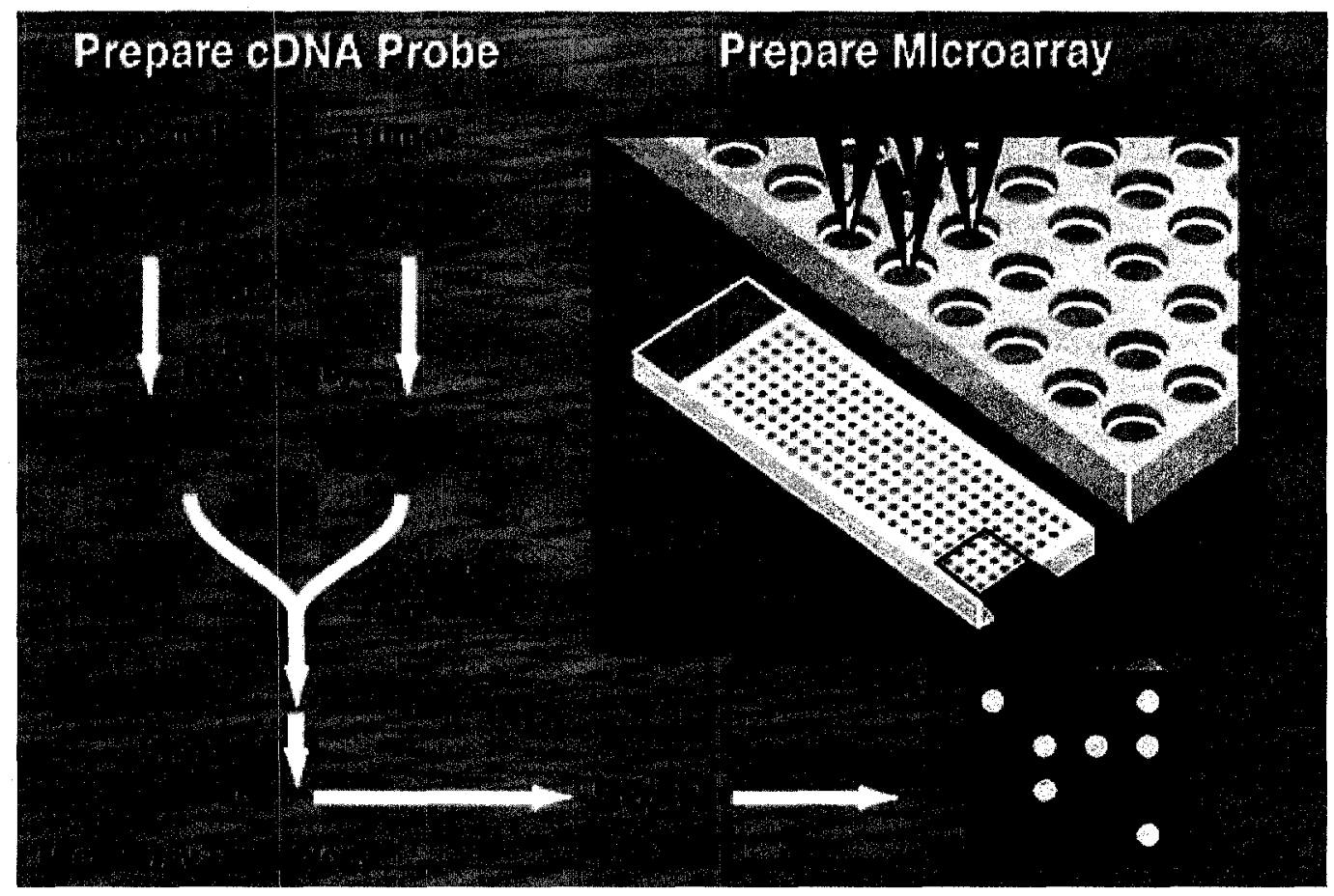

Figure 1.4, cDNA microarray scheme. Graphics from http://www.accessexcellence.org.

\subsection{Microarray Time Course Experiments}

Microarray time course experiments with genome-wide gene expression profiles provide a unique opportunity to study biological signal processing among genes at the transcription level. In response to different environmental conditions, a cell system alternates the transcription of different genes to coordinate its adaptation process based on programmed logic stored in its genome. These organized responses provide a unique signature of cellular response to a specific environmental condition at the transcription level and hence is of major biological interest. Since the signals sent from a specific gene may only be present for a short period of time, time course microarrays provide very 
important information to study cellular signaling processes. As an example, the temporal expression profiles for 8 genes in an experiment to examine the effect of infection of $A$. phagocytophilum on polymorphonuclear leukocytes (PMNs or netrophils) (Borjesson et al., 2005) are shown in Figure 1.5. This data set is analyzed in chapter 6.

There is increasing interest in microarray time course experiments and several statistical models have been proposed for time course data in recent years, among them the two-way ANOVA model (Park et al., 2003), B-spline model based time curve fitting (Bar-Joseph et al., 2003; Luan and Li, 2004), multivariate empirical Bayes method (Tai and Speed, 2006), and hidden Markov models (HMMs). Schliep et al. (2003, 2004, 2005) used partial supervised learning HMMs to cluster gene time course profiles. Their method uses a group of HMMs to represent clusters estimated from the temporal profiles of a known group of genes, then iteratively assigns unknown genes into these clusters and refines the clusters. The order (i.e. the number of hidden states) for the HMM in each cluster is either predetermined or can be inferred from the genes in a training set. Yuan and Kendziorski (2006) proposed another HMM in which the order is specified as a function of the number of treatment conditions. Existing HMM methods assume that the order is either already determined by some imposed criterion or is the same as those of known genes. This may not always be the case. The biological processes generating the observed expression for different genes are not known and may potentially provide important biological insight for cell systems. The order can not be assumed to be a known parameter; instead needs to be investigated. The estimated order under one particular treatment condition may provide important information about regulatory interactions among genes. 

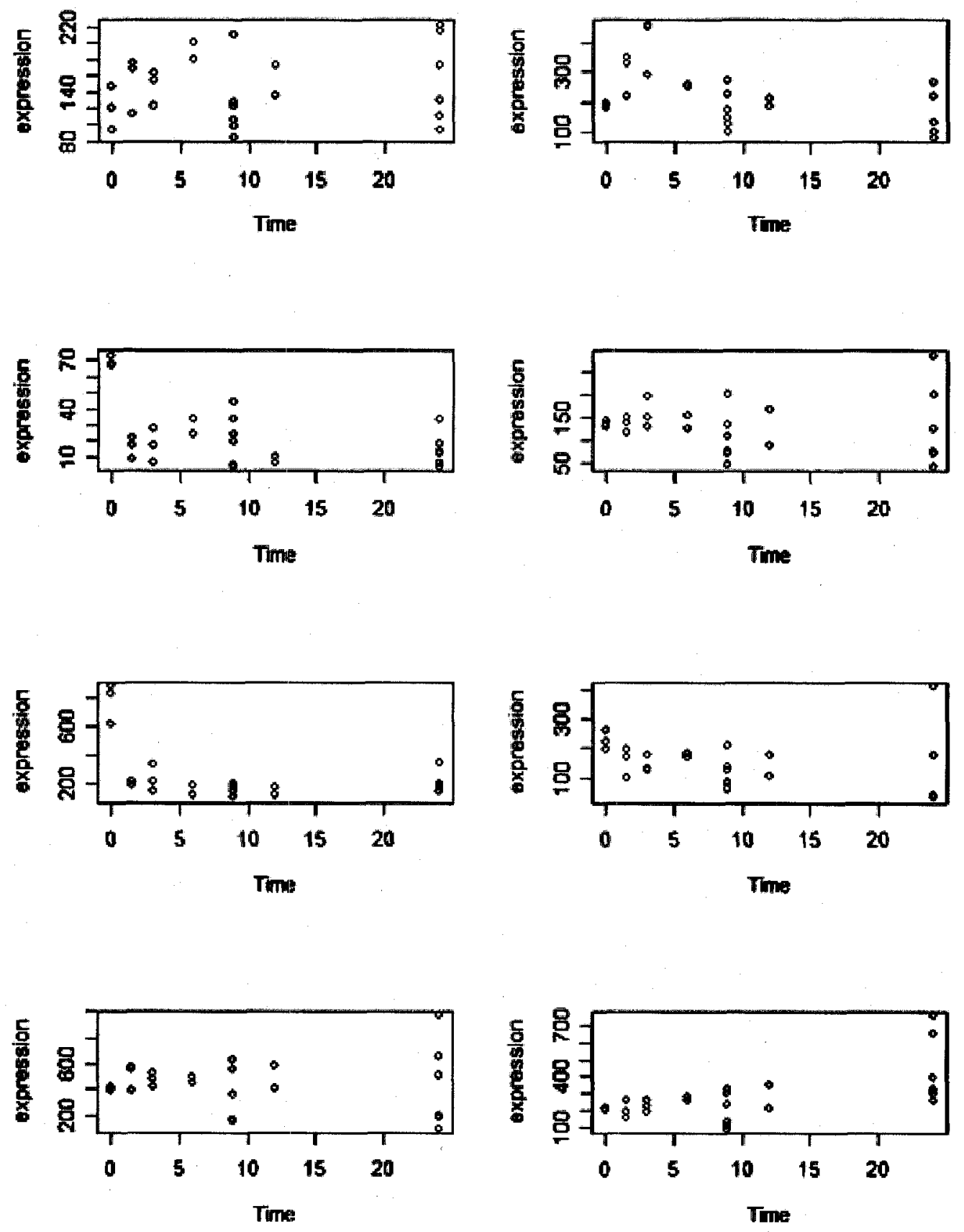

Figure 1.5, Gene expression profiles for 8 genes at time points $0,1.5,3,6,9,12$ and 24 hours. 
To investigate cell-wide gene expression profiles for a large number of genes, biologists usually measure gene expressions repeatedly over time. This kind of experimental setting is described in Figure 1.6. Our further discussion will focus on the situation where the same type of cells are prepared under different treatment conditions. In other situations, the treatment condition may be kept the same, while different types of cells are measured. We discuss the first situation here only since it is a typical microarray experimental setting.

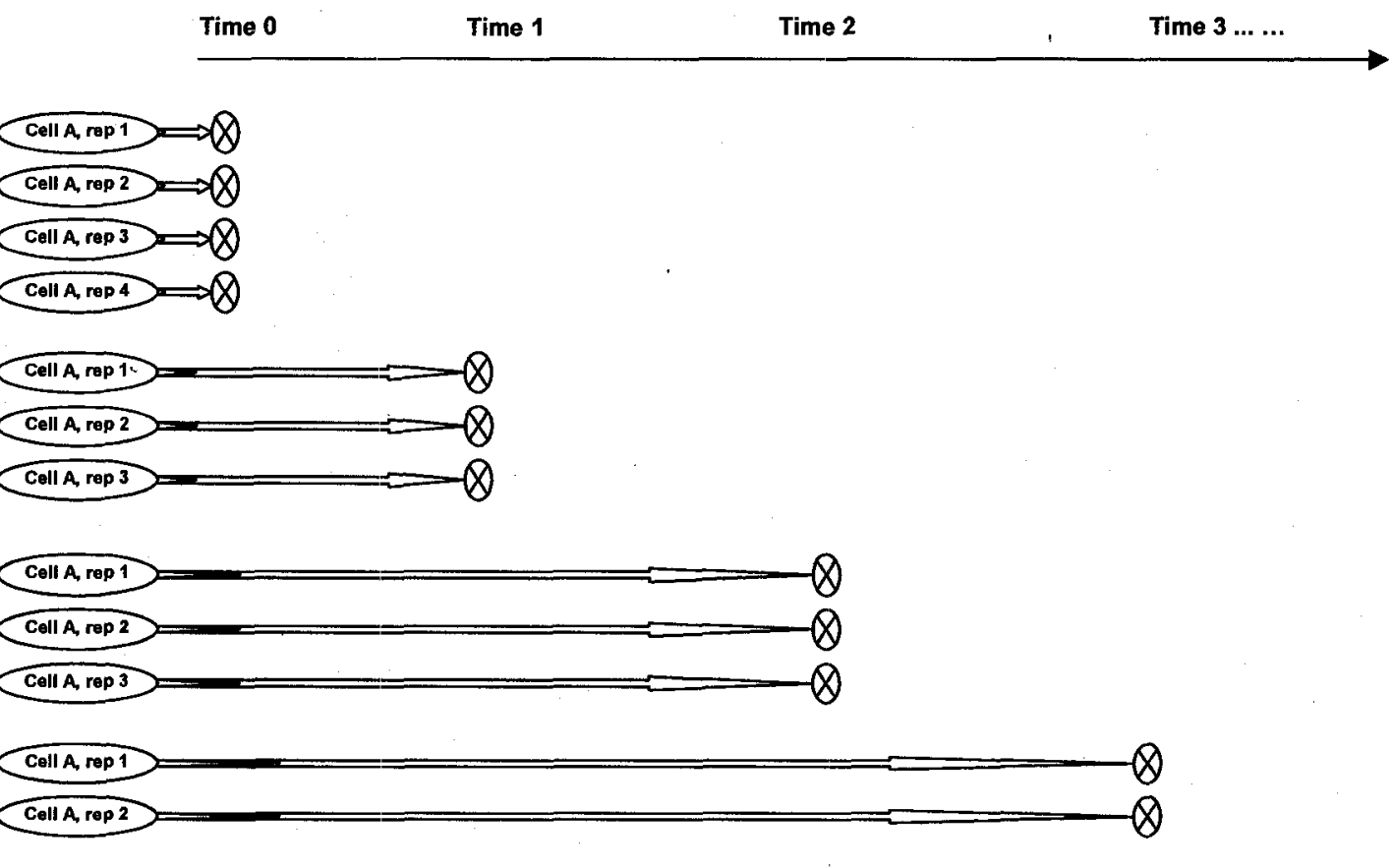

Cell is lysed and gene expressions are measured

Figure 1.6, Microarray time course experiment under one specific treatment condition.

As shown in Figure 1.6, individual cells from the same biological type under one specific treatment condition are lysed at a given time point. Their gene expression levels 
are measured for a given list of genes. Normally, the gene list is fixed throughout the whole time course experiment. This list is usually determined by the design of the microarray to cover most important genes in the genome. The list typically consists of a large number of genes. At a particular time point, the same microarray experiments are performed several times. The expression levels of the genes are recorded as replicates for a specific time point. The replicates here are the biological replicates because the replicates come from different cells as shown in Figure 1.6. The number of time points is normally chosen to be 5 to 10 . The number of replicates could be different across time points.

One important effort for experimentalists is to synchronize the cell so that at each time point, the cells undergo the same stage of the biological process. The biological process which generates a gene's expression profile is of primary interest and is also the essential motivation for the development of our analysis method. As commonly agreed, the interaction relationship among genes is usually represented by a gene regulatory network. The structure of this network, however, may change along with treatment conditions and over time. To illustrate the cause and effect structure, two simplified conceptual diagrams for a cell system consisting of only six genes are presented in Figure 1.7 and Figure 1.8. They are used to represent two treatment conditions as an example. 


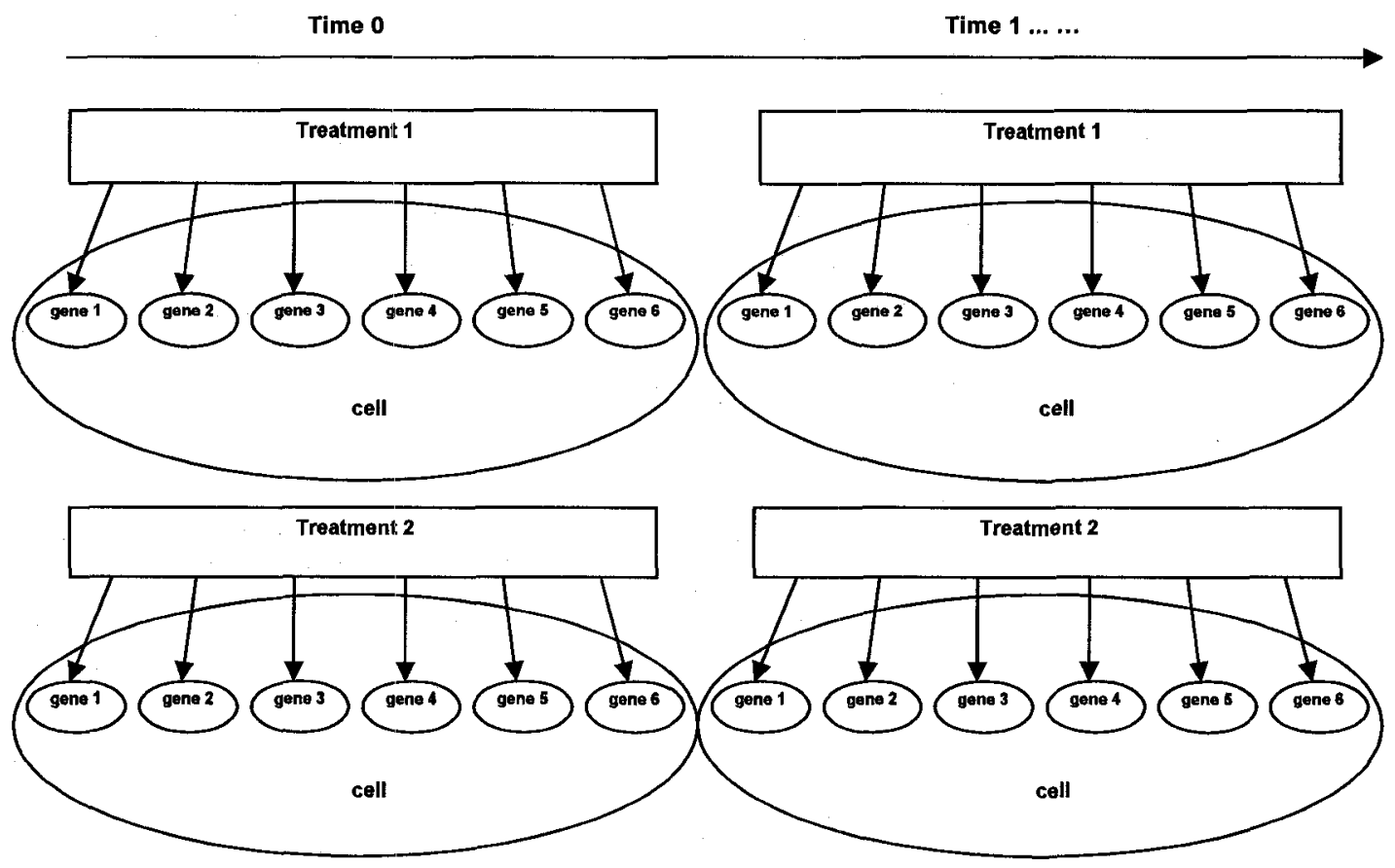

Figure 1.7, First category of assumptions: the effect of treatment condition is assumed to be able to reach every gene of a cell. The same treatment is assumed to have constant effect on each gene across time. Under the constant treatment effect over time, the change of the expressions is usually attributed to the time effect for a gene.

There are at least two different categories of assumptions about the underlying biological process which generates the gene expressions. The first one, as illustrated in Figure 1.7, is the most often used. It assumes that every gene can be affected directly by an external treatment condition controlled by a human experimentalist. The arrows in Figure 1.7 represent the effects exerted from the cause to the affected items. Different treatment conditions are assumed to be able to reach each gene directly in the same way. The influence from a treatment condition to a gene is commonly assumed to be the same across time. Under the same treatment, the change of expression of a gene is regarded 
sometimes as the result of a time effect. Unfortunately, this category of assumptions is not supported by existing biological evidence. The well observed regulatory interactions among genes make those analyses based on these assumptions problematic.

Instead, the gene regulatory network discovered so far strongly suggests the second category of assumptions, as shown in Figure 1.8. Under this category of assumptions, under one treatment condition, each gene can be influenced by the external condition of a cell directly, or by the effect of other genes or a mixture of both. For example, in Figure 1.8, at time point 0 under treatment condition 1, gene 1 is affected directly by treatment condition 1 . Gene 3 is not directly affected by treatment condition 1 since there is no arrow connecting treatment 1 to gene 3 . However, it is indirectly affected by treatment condition through the influence of gene 1 . Gene 2 receives both direct and indirect influences. The causal relationship among genes can also change with treatment conditions, and with time under the same treatment condition. As shown in Figure 1.8, the arrows and their connected genes may be different at time 0 between treatment 1 and treatment 2 . Under the same treatment condition, the arrows and their connected genes can be different between time point 0 and time point 1 . In other words, at the same time point, different treatment conditions may affect different genes. In case a fixed set of genes is used by cells to sense different treatment conditions, their activated genes could be different. Furthermore, these activated genes may be different from time to time. 


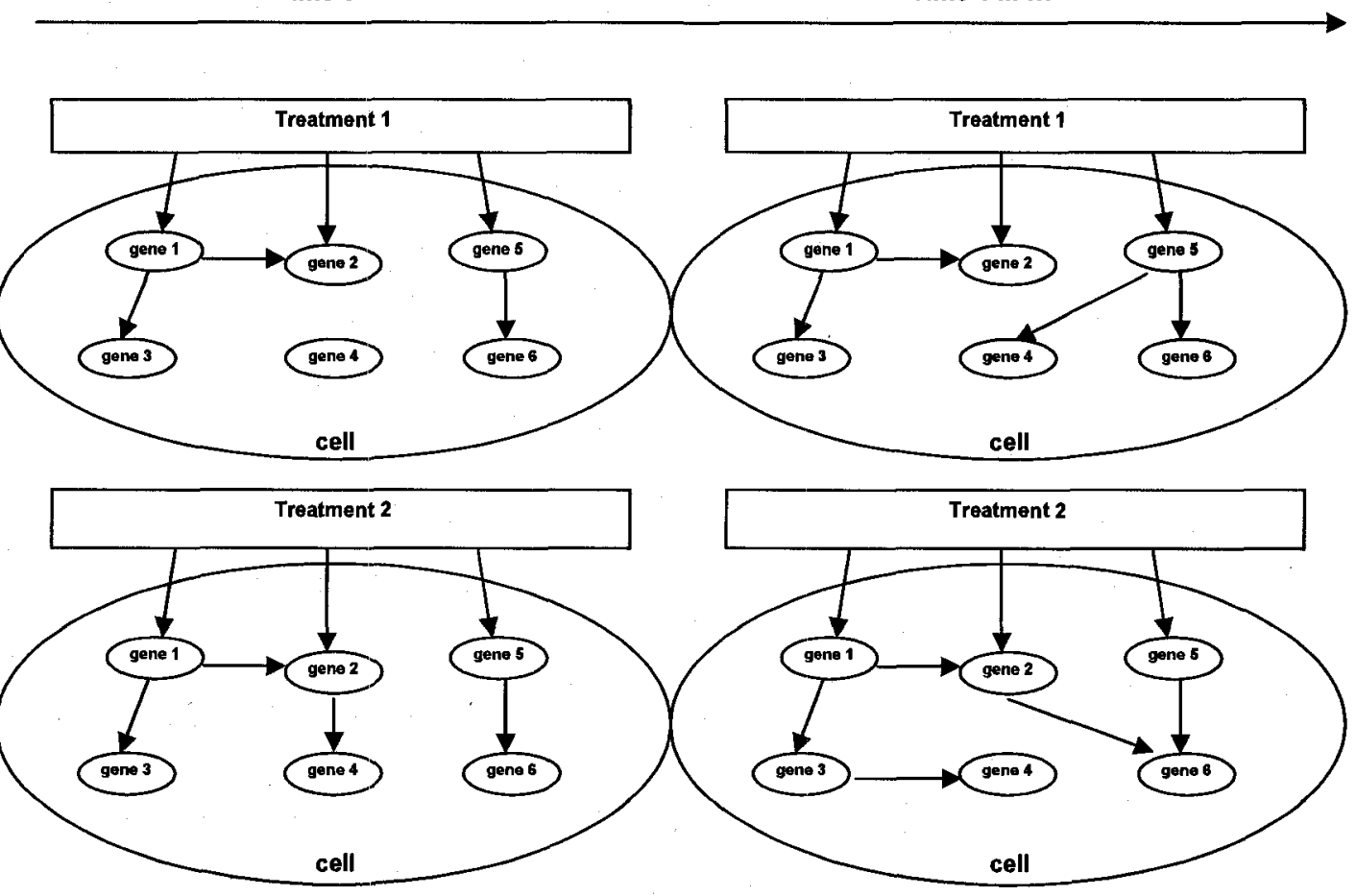

Figure 1.8, Second category of assumptions: the treatment condition is not able to reach all the genes in a cell. Different treatment conditions may affect different genes. The genes affected by a specific gene may be different from time to time.

The key difference between the above two types of assumptions is that it is acknowledged only in the second category of assumptions that a treatment condition can only be controlled to the cell level instead of to the gene level. In a real situation, which gene is affected directly by treatment condition is not known. Given a treatment, which gene activates another gene is not known either. Because this kind of knowledge is of primary biological interest, the analysis assuming the interaction is ignorable is inappropriate. Moreover, how a controllable experimental condition affects the regulatory 
relationship is not known and thus is not controllable. The fact that genes are not equally affected by one particular external condition of the cell, and that some genes depend on other genes for information about external conditions is well established in biology. In addition, the cascading of cellular signals also results in different times at which a treatment condition affects particular genes through other genes. Regulatory interactions, such as the turning on or turning off of the expression of a gene, are not fully deterministic and uncertainty can be reasonably assumed. Hence, the observed gene expression level is affected potentially by two confounding effects. The first effect is the controllable treatment condition. The second one is the uncontrollable gene regulatory effect. The effect produced from other genes that changes the expression of a specific gene is referred to as the gene context effect. For a particular gene, the gene context effect is potentially gene specific under the same treatment. It may also be different for the same gene under different treatments.

In Figure 1.8, the arrows coming from the treatment condition represent the controllable treatment effects. The arrows between the genes inside each cell represent the uncontrollable gene context effects. The gene context effect is not deterministic. In particular, the higher expression of one specific gene may not be due to a treatment condition. Instead, it may have resulted from random activation by one of its regulatory genes. The regulatory network among genes is not fully understood yet and is of primary research interest, making the first category of assumptions which ignores the effect of interaction among genes undesirable. 
An often-used approach of microarray analysis is aimed to compare the expression levels between different treatment conditions and to find those genes which are differentially expressed. For the same consideration, most time series microarray analysis is aimed to compare the expression profiles between treatments to see whether the temporal expression pattern is different or not. This kind of analysis is problematic because it is based on the first category of assumptions. To see the problem, suppose the activation (activation means a higher level expression) of gene 1 will lead to the activation of gene 3 as shown in Figure 1.8. Because the regulatory relationship is not deterministic, the probability that gene 3 is activated, given that gene 1 is activated, is less than 1 .

$$
P \text { (gene } 3 \text { activated } \mid \text { gene } 1 \text { activated })<1
$$

This means that there is a non-zero probability that gene 1 fails to activate gene 3 under a given treatment condition 1 . If gene 1 is always activated under treatment condition 1 , each realization of gene 1 can be used to characterize treatment condition 1 . However, each realization of gene 3 is not guaranteed to come from its activated state because the probability given gene 1 is activated is less than 1 . Therefore, the expression measured for a gene like gene 3 under treatment condition 1 can not be guaranteed to come from its activated state in each realization. For this kind of gene, the comparison of the expression levels between two treatments can not be used as a characterization of treatment condition 1 because its expressions may be generated when it is activated or inactivated under a specific treatment condition.

To further illustrate this, let us assume there are two expression states for gene 1 and gene 3. Also let us assume that the activated state is the state with higher expression 
levels and the inactivated state has lower expression levels for both genes. If the realized expressions for gene 1 under treatment 1 are always from the activated state (higher expressions), the realized expressions for gene 3 under treatment 1 can come from either the activated state or the inactivated state because the probability in (1.1) is less than 1 . In other words, in some situations gene 1 fails to activate gene 3 even though they are always under treatment condition 1 . The comparison of the expressions for gene 3 between the two treatment conditions is not meaningful because the replicates under each treatment condition are potentially a mixture from both activated and inactivated states. In a real situation, because we do not know a gene's position in the pathway for a treatment condition, statistical comparisons conducted to look for the differential expressions may simply generate misunderstanding.

For the same reason, the temporal expression curves or the state sequences estimated based on time course expression profiles should not be used in the comparison of treatments. Without knowing the position in a pathway for a particular gene, the comparison for the expression at each time point among treatments has the same problem as mentioned above. That is, the realization of expressions at specific time points may arise from either of the states.

From above we can see that whether the realizations of a gene's expression comes from more than one state depends on its position in a pathway or depends on how it relates to other genes. The probability of visiting more than one state could be different for different genes, and it may differ with treatment conditions. This is the reason why the effect is referred to as the gene context effect. The gene context effect thus can be 
thought of as a modifier of the effect from a specific treatment condition, making it different for different genes. Understanding the gene context effect will lead us to discover the regulatory relationships among genes and this is also the primary goal of our method.

To tackle the challenging problem caused by gene context effects, we propose a new approach for the statistical inference on microarray time course data. We propose to identify the response genes first. These response genes could be genes which are involved in sending out regulatory signals or performing certain biochemical functions or a mixture of both. As response genes, they all have a common characteristic: their expressions are generated from different states over time. We define response genes as those genes which have shown evidence that they have occupied different states of expression. The detailed definition of expression state will be discussed in the following chapters. Those genes influenced by the gene context effect may show expressions realized from different states. By extracting this kind of gene, we can obtain information about those genes which interact with each other under a specific treatment. This knowledge, accumulated across many different treatment conditions, can lead us to discover the gene regulatory network.

The different states (for example, activated state or inactivated state) have their own molecular biological meaning too. Based on current biological knowledge, the transcriptional responses are all performed by employing molecular association to targeted molecular complexes, that is, by binding to certain sites. In a general sense, binding means molecular binding, which is the association of two or more molecules or 
molecular complexes. Molecular binding is an attractive interaction between two molecules or molecular complexes (formed by association of two or more molecules). The molecules that can participate in molecular binding include proteins, nucleic acids, carbohydrates, lipids and other organic molecules of a cell. The formation of binding during gene interaction and cellular signaling processes suggests the following characteristics. First, the binding or other responses (e.g. no binding, binding or some different types of binding) are achieved by changing the expression levels. For example, the shift from a lower expression to a higher one may result in an activation signal sent to the genes it regulates. Note that a specific expression level itself such as a higher expression does not always correspond to activation for different genes. Second, the responses or the regulatory signals that can be communicated among genes are discrete in nature, and the number of different states or responses is limited. The status of binding or different types of binding is less likely to be continuous. Third, without accurate expression level detection and control mechanisms in cells, the binding or release of binding in a cell system can only be achieved probabilistically rather than deterministically. Of course, the certainty of a signal is increased if the expression level change is sufficiently large from its original expression intensity. However, the expression level change may not be necessarily large enough to meet a statistical significance level imposed by analysts.

The focus of our proposed method is to identify the set of response genes that change state under a treatment condition. We will show that state differentiation will lead us to find those genes that are used by a cell system in response to a specific treatment condition. In the following analysis, the temporal expression is conceptually represented 
by a hidden Markov model (HMM). The HMM is specified for each gene under each treatment condition separately. We postulate that there is a finite number of hidden states representing the different molecular binding status, such as binding, not binding or different kinds of binding. The status of binding can not be directly observed from microarray measures and therefore is represented by the hidden states of the model. To make the signal detectable for other genes or to alter an on-going biochemical process, the states need to be stochastically distinguishable. At each time point, the observed expressions are regarded as being generated from a distribution which depends on the corresponding state at that time point. Different genes under different treatment conditions may employ different hidden states to perform biological functions. These states are not directly comparable. Instead, the differentiation of the states provides the only basis for identification. The estimation of the number of hidden states, also referred to as the order of HMMs, provides the most important information for us to understand gene context effects.

There are two dependence structures in our hidden Markov model. First, at each time point, the gene's expression level depends only on the corresponding hidden state. This dependency is modeled by the so called emission distribution. Second, at each time point, the state is influenced only by its immediate previous state. This temporal dependency is modeled by the transition probabilities. For the reasons discussed above, we assume that the Markov chain of the hidden states has a finite and discrete parameter space. The underlying Markov process is also assumed to have a stationary probability when we perform the order estimation. The reasons of using a stationary probability will be discussed below. 
Those genes with more than one state consist of the subset of genes which are used by a cell in response to a particular treatment condition. For each treatment condition, our analysis provides a subset of response genes which show evidence of having different hidden states. The rest of the genes are in the non-response gene subset. Our method provides essential information to compare the response gene set (or the nonresponse gene set) across any number of treatment conditions without the need to align the time points. Those genes having high frequency to appear together may be involved in the same or closely related biological pathways.

Estimating the order is also the most straightforward way to find the response genes. Estimating the state sequence on the other hand requires that more parameters be estimated, making it less efficient for microarray analysis where a typical limited sample size is always the case. Hence, we suggest estimating the order instead of estimating the state sequence to find the response gene set. In addition, because the state sequences can not be used for the comparison between treatments, it does not provide any informational gain beyond the order in characterization of the gene context effect.

For each gene, we further assume that the observed expressions at a specific time point come from the same hidden state for a specific treatment condition. Usually one of the objectives of experimentalists is to keep all cells involved in time course microarrays synchronized so that the cells are reasonably regarded as undergoing the same stage of a biological process. With a limited number of replicates and time points, it is really 
difficult to find the number of hidden states in a non-synchronized situation. We focus on the synchronized situations hereafter and assume this condition is satisfied.

The limited sample size and the limited number of time points are the reasons why microarray experiments are used as a screening tool to narrow down a subset of genes which can be further investigated in more detail. Our proposed method is developed according to this purpose. The subset of response genes provides the genes for more detailed examination. In addition, our method is an estimating method. It therefore provides flexibility to identify response genes at different response intensity levels.

\subsection{Microarray data pre-processing}

In most situations, before a statistical analysis is performed for microarray data, the raw expressions need to be pre-processed to reduce the unwanted variation resulting from the multiple steps in a microarray experiment. The quantification of the fluorescence intensities consists of multiple steps such as locating the spot and measuring the spot color intensity using image analysis techniques (Schadt et al., 2000; Yang et al., 2000; Nguyen et al., 2002).

At the first step, each spot needs to be located. The intensity of either one color for oligonucleotide arrays or two colors for spotted arrays is measured. The background color intensity around the spot is also measured. The background color intensity is usually subtracted from the spot intensity to estimate the actual intensity for each spot. 
When there is more than one spot corresponding to the same probe, the intensity measures of these spots are averaged. These averaged intensity values are commonly used as the input data for the statistical analysis of gene expression.

The background color subtraction sometimes results in a negative intensity value, indicating the spot measured may have some quality issues. Two common practical ways to deal with this are either to discard the data for these spots or to set these values to an arbitrary constant slightly bigger than zero.

Another important consideration in microarray data pre-processing is to choose the sampling distribution to describe the data. There are two common choices for the intensity based on the well observed skewness of intensity data: log-normal distribution and gamma distribution (Kerr et al., 2000; Newton et al., 2001; Lee et al., 2000; Black and Doerge, 2001). For the log-normal distribution, a log transformation of the data is sometimes used. The $\log$ transformation is used quite often when inference is targeted on the fold change between two treatment conditions. The transformation techniques were developed with a focus on variance stabilization so that the statistical inference of fold changes could be expected to be more efficient. As discussed above, the approaches for conducting statistical tests on fold change are based on the first category of assumptions. These assumptions imply that the mean expression level can be used as a proper characterization of a treatment condition. Such simplification may not be valid when the gene context effect and treatment effect both have potential impacts on the expression intensity. 
Since our focus here is to model the expression distribution directly, we choose to follow the direction of Newton et al. (2001) and use the gamma distribution without any data transformation. The gamma distribution is used by many researchers in modeling microarray data (Chen et al., 1997; Ideker et al., 2000; Newton et al., 2001; Kendziorski et al., 2003). Its fitting is examined by Newton et al. (2004) using QQ plots of the expressions of genes with similar mean expressions. The result confirms that the gamma distribution is a good choice for microarray data.

An additional step to remove other potential sources of variation is referred to as normalization (Nadon and Shoemaker, 2002). In addition to background intensity, there are other variation sources, caused by differences in hybridization conditions, such as temperature or humidity, when the experiments are conducted at different times. Additionally, laser power differences during the scanning may also produce differences in the intensity readings. It is therefore considered a necessary step to normalize the data across arrays when different arrays are used in an experiment. Typical approaches for normalization focus on standardizing overall intensity by fitting a loess curve and looking at the residuals and the MA plots (an MA plot is a plot of log-ratio of two expression intensities versus the mean log-expression of the two) (Dudoit et al., 2002). An alternative approach is the quantile normalization method (Bolstad et al., 2002), which transforms each of the array-specific intensity distributions so that they all have the same quantiles. The data set we analyze in chapter 6 is pre-processed and normalized by GeneSpring package version 6.0. The expressions among probe pairs are first averaged and the mean expression is obtained for each probe set. The expressions for different 
probe sets are normalized for each array to produce the input data for our statistical analysis.

The pre-processed data from the study of Dorjesson et al. (2005) are used in the analysis in chapter 6 . The data pre-processing and normalization were performed using GeneSpring package 6.0 in the original study. The GeneSpring package from Silicon Genetics provides data pre-processing and analysis tools for both Affymetrix microarray and cDNA microarray data. The normalization procedures provided in GeneSpring are similar to those of Affymetrix MAS 5. For the data set analyzed in chapter 6, the averaged difference between a perfect match and mismatch for each probe set is normalized by a per-chip normalization using the distribution of all probe sets on each chip. Similar to the global scaling procedure of MAS 5, per-chip normalization centers the intensities of each chip to a constant to control for the chip-wide variations. In addition, the per-gene normalization is applied using the median intensity of each gene to control for differences in detection efficiency among spots.

Pre-processed microarray data are then inputted for further statistical analysis. As discussed above, because of the confounding of treatment effect and gene context effect, the usual statistical tests for differential expression are questionable. Instead, we propose to estimate the order to find the subset of response genes. In chapter 2 , we introduce our general model and notation. In chapter 3, the empirical Bayes estimation of gene specific coefficient of variation (CV) and the shape parameter of the gamma component distribution is discussed. In chapter 4 , the order is estimated for each gene. The simulation study of order estimation is discussed in chapter 5 . In chapter 6 , we analyze a 
real microarray time course data set GDS1428. This experiment investigates the effect of infection of A. phagocytophilum on Polymorphonuclear Leukocytes. We find that the order is both gene specific and treatment specific. The comparison of response gene set between the two treatment conditions is also described. In chapter 7, we include conclusions and further discussion. 


\section{Chapter 2}

\section{GENERAL MODEL}

Hidden Markov models are widely used in modeling various stochastic processes over time (Rabiner, 1989; MacDonald and Zucchini, 1997). The underlying biological process produces the observed gene expressions. This process is potentially gene specific based on the second category of assumptions introduced in previous chapter. The expression level is obviously continuous, while the underlying biological responses can be reasonably assumed to be finite and discrete in nature.

The hidden Markov model has two stochastic components. One is the hidden Markov chain, which has temporal dependency structure described by the transition probabilities. The other component is the observed expression levels which are assumed to depend on the hidden state occupied at the corresponding time point. The observed gene expressions at a time point are thought of as being generated from a gamma 
distribution whose mean parameter is determined by the hidden state at the corresponding time point. The gene specific shape parameter is assumed to be the same across time under one particular treatment condition. Letting the mean parameter depend on the corresponding hidden state is because the signaling or the response of a gene is usually achieved by alternating the expression levels based on available biological evidence. Note that a specific hidden state alone (whether it represents a high expression level or a lower expression level) does not indicate a definitive function. And the activation (or a higher expression level) has different functions for different genes. Thus the state itself alone does not provide useful information to characterize a gene's behavior. Instead, a response as defined as a change of states can be used in characterizing a treatment condition.

The shape parameter for a specific gene represents the biological variation across individual cells from the same cell type under a treatment condition. It is observed that some certain type of cells under a specific external condition can display higher variability so that the variation among observed gene expression replicates could be large. However, we have no evidence showing that this kind of variation changes dramatically over the time under the same treatment condition. Accordingly, the shape parameter is assumed to be the same across time for each gene. To account for potential gene to gene differences, we allow the shape parameter to differ among genes.

The number of hidden states for each gene (also referred to as the order of the hidden Markov chain) provides important information to characterize gene context effect under a treatment condition. The estimation of the order for microarray time course data, 
to our knowledge, has not been investigated so far. The number of hidden states can also provide information in understanding the nature of biological signals in a cell system. If the expression profile is believed to be generated from a single distribution, the gene is probably a gene that does not send out a signal or does not respond to an ongoing process. Those genes with more than one state are probably responding or signaling and thus can be used to investigate the interaction relationship among genes. The set of response genes are those genes which are used by a cell to organize its response to a specific treatment condition. These genes exchange signals with each other to perform certain biological functions so that the cell can adapt to its environment. Some of these response genes can be the same for different treatment conditions because a cell may employ a portion of a pathway repeatedly to respond to different conditions. This is also supported by biological evidence that certain essential biological functions are performed under different environmental conditions.

Furthermore, the whole set of response genes is the characterization of the response for a cell. The information about interactions among genes is contained in the response set in terms of co-occurrence of other genes. Thus the information regarding gene interaction is contained not only in the appearance of a single gene but also in cooccurrence with other genes in the response set. The signal or response of one gene is characterized also by the response of its regulated genes. In other words, the signal sent out by a gene should be judged by the response of its regulated genes instead of by a criterion from human observers. In this sense, the set of response genes as a whole is the signature of a cell's response toward an external condition. It is therefore important for us to report the whole set of response genes when a real data set is analyzed. 
The notation for a typical microarray time series experiment can be described as follows: there are $G$ genes measured in one microarray under a specific treatment condition. For each gene, there are $T$ time points measured. $T$ is usually the same for all genes under one condition. The replicates at different time points need not be equal, but for notational convenience, we will assume that for gene $g$, at a time point $t$, we have $R$ replicates. Our method does not require equal replicates. Let $\boldsymbol{Y}_{g t}=\left(y_{g t}, y_{g t 2}, \ldots \ldots, y_{g t R}\right)^{\mathrm{T}}$ denotes the observed expressions for gene $g$ at time point $t$ with $R$ replicates. The total number of observations is $n$. In case $R$ is equal for all time points, the total number of observations $n$ is equal to $R T$.

Let $Z_{g t}$ be the hidden state at time $t$ for gene $g$. The conditional distribution of $Y_{g t}$ given $Z_{g t}$ does not depend on $t$. The $\left\{Z_{g l}, Z_{g 2}, \ldots \ldots, Z_{g t}, \ldots, Z_{g T}\right\}$ denotes the sequence of hidden states in a discrete state space. The subscript $g$ indicates that the hidden states are gene specific, which can be defined differently for different genes. Since our HMM and its order are always estimated for each gene separately, we drop the subscript $g$ hereafter. The observed expressions at a specific time point can be thought of as being generated from the corresponding emission distribution determined by the hidden state. The expression replicates at one specific time point are generated from the corresponding distribution independently.

The following conditions are assumed to be satisfied for the HMM considered subsequently when the order estimation is our primary objective:

1. The hidden state $Z_{t}$ has stationary probabilities $\pi_{k}$, for $k=1,2, \ldots, K$. 
2. Conditioning on $Z_{t}$, the observed expression collection $\boldsymbol{Y}_{\boldsymbol{t}}$ at time point $t$ is independent of observations at other time points: $\boldsymbol{Y}_{1}, \boldsymbol{Y}_{2}, \ldots, \boldsymbol{Y}_{(t-1)}, \boldsymbol{Y}_{(t+1)}, \ldots \ldots, \boldsymbol{Y}_{T}$ and is also independent of the hidden states at other time points: $Z_{1}, Z_{2}, \ldots \ldots, Z_{(t-1)}, Z_{(t+1)}, \ldots \ldots, Z_{T}$. 3. The replicates at one specific time point $\boldsymbol{Y}_{t}=\left(y_{t 1}, y_{t 2}, \ldots, y_{t R}\right)^{\mathrm{T}}$ are independent of each other, given the corresponding hidden state $Z_{t}$.

In estimating the number of hidden states or the order of an HMM for each gene, the stationary probabilities for the hidden Markov chain are denoted by $\pi=\left(\pi_{1}, \pi_{2}, \ldots \ldots\right.$ $\left.\pi_{K}\right)^{\mathrm{T}}$, where $K$ is the order of hidden Markov model. This stationary distribution is commonly assumed in order estimation for HMMs by existing methods (Poskitt and Zhang, 2005; Lindgren, 1978; Leroux and Puterman, 1992; Ryden, 1995; MacKay, 2002). Poskitt and Zhang (2005) argued that the theoretical analysis based on the joint density of observed data and hidden states is extremely difficult, while there are substantial computational gains if inference is based on the marginal distribution of observations using the stationary probability. Here we follow this direction to assume a stationary distribution for the unobserved Markov chain and estimate the order based on the marginal distribution of observed data.

Under above assumptions, the observation $Y_{t r}$ can be thought of as coming from a mixture density function where the stationary probability corresponds to the mixing proportion of the finite mixture model:

$$
f_{K}\left(y_{t r} \mid \underline{\pi}, \underline{\mu}, \alpha\right)=\sum_{k=1}^{K} \pi_{k} h\left(y_{t r} \mid \mu_{k}, \alpha\right)
$$


where $h\left(y_{t r} \mid \mu_{k}, \alpha\right)$ is a family of gamma distributions defined in (2.2), $\underline{\pi}=\left(\pi_{1}, \ldots, \pi_{K}\right)^{T}$ and $\underline{\mu}=\left(\mu_{1}, \ldots, \mu_{K}\right)^{T}$ It is also referred to as the emission distribution in the context of HMM's. The gene specific shape parameter $\alpha$ will be estimated by an empirical Bayes approach in the next chapter. The value of $\mu$ in a one dimensional parameter space, is allowed to vary across time and is expected to be different for different genes. For the emission density or the mixture component $h\left(y_{i r} \mid \mu_{k}, \alpha\right)$, the mean parameter $\mu_{k}$ is determined by the corresponding hidden state $Z_{t}$ at time point $t$, while the shape parameter $\alpha$ is the same across all time points. Different genes may have a different shape parameter $\alpha$.

As a stationary probability is assumed in estimating the order of a HMM, our method does not require the equal time interval in the microarray time course data. The simulation data sets in chapter 5 are also generated from finite mixture models.

The gamma distribution used as the mixture component distribution for each gene at time point $t$ is parameterized as follows:

$$
h_{t}\left(y_{t} \mid \alpha, \mu_{k}\right)=\frac{1}{\Gamma(\alpha)}\left(\frac{\alpha}{\mu_{k}}\right)^{\alpha} y_{t}^{\alpha-1} \exp \left(\frac{-y_{t} \alpha}{\mu_{k}}\right)
$$

where $\mu_{k}$ is the mean at at specific time point $t$ corresponding to state $k$. Different hidden states have different $\mu_{k}$ values which depend on the corresponding hidden state $Z_{k} . \alpha$ is the shape parameter. With this setting, $E\left(y_{i}\right)=\mu_{k}, \operatorname{Var}\left(y_{i}\right)=\mu_{k}^{2} / \alpha$ and $C V=1 / \sqrt{\alpha}$. The $C V$ is the gene specific coefficient of variation. $\alpha=1 / C V^{2}$ can be thought of as a squared stability indicator. 
Another parameterization of the gamma distribution, which will be also used in the following chapters, is

$$
h_{t}\left(y_{t} \mid \alpha, \beta_{k}\right)=\frac{1}{\Gamma(\alpha) \beta_{k}^{\alpha}} y_{t}^{\alpha-1} \exp \left(\frac{-y_{t}}{\beta_{k}}\right)
$$

where $\beta_{k}$ is the scale parameter at a specific time point $t$. Different hidden states have different $\beta_{k}$ values which depend on the corresponding hidden state $Z_{t} . \alpha$ is the shape parameter. With this parameterization, $E\left(y_{t}\right)=\mu_{k}=\alpha \beta_{k}, \operatorname{Var}\left(y_{t}\right)=\alpha \beta_{k}^{2}, C V=1 / \sqrt{\alpha}$. The above two parameterizations of the gamma distribution are used for the empirical Bayes estimation of the shape parameter in chapter 3 and order estimation in chapter 4. 


\section{Chapter 3}

\section{EMPIRICAL BAYES ESTIMATION OF THE SHAPE}

\section{PARAMETER}

Empirical Bayes and Bayesian hierarchical models have become popular approaches in recent years for microarray analysis. The typical small sample size both for non-time course experiments and time course data is probably the reason. Trying to take advantage of the large number of genes measured, Bayesian models seem to provide a possible way to borrow information from other genes. Another reason to consider a Bayes approach is the well observed heterogeneous variances among genes with different expression levels. To use a common variance for all genes is obviously problematic, while gene specific variance estimation is very difficult with such a small sample size. Many suggest borrowing information across genes in estimating expression means or variances. This strategy is hard to justify because the mean or variance for different genes may differ dramatically without any evidence that they share any common pattern. Information borrowing requires the assumption that the genes are sharing a common pattern in terms of mean or variance. Without the support of experimental evidence that 
a common pattern is shared based on the mean or variance, the information borrowing approach only results in the introduction of irrelevant information.

There is no evidence indicating that the mean or variance shares a common pattern. However, for the ratio of the standard deviation over mean or the coefficient of variation $(\mathrm{CV})$, there does exist evidence that a similar $\mathrm{CV}$ is shared across different genes (Chen et al., 1997; Ideker et al., 2000; Baggerly et al., 2001; Li and Wong, 2001; Rocke and Durbin, 2001; Theihaber et al., 2001; Tsodikov et al., 2002; Newton et al., 2001; Baldi et al., 2001; Newton et al., 2004). This is also one of our considerations to use a gamma distribution to model the expressions because the gamma distribution takes into account that the variance increases as mean increases. The gamma model also has past success in modeling continuous abundance data as suggested by Dennis and Patil (1984). In addition, as discussed by Durbin et al. (2002), at higher expression levels, the standard deviation of expression intensity varies linearly with the mean, at low expression levels, log transformed data have a potential inflation effect on the variance. The log-normal distribution may have the same problem at low expression levels.

The original use of the gamma distribution in microarray data assumed the same shape parameter for all the genes in data set. This may be too strict and can be relaxed by using a hierarchical Bayesian model as proposed by Lo and Gottardo (2007). Instead of assuming the same shape parameter or CV for all genes, we allow the shape parameter to be different across genes. As proposed by Lo and Gottardo (2007), we postulate that the shape parameters of different genes are generated from a prior distribution. Because there is no evidence indicating that the sample variability (described by $\mathrm{CV}$ or shape parameter) 
changes over time for the same gene, the $\mathrm{CV}$ is assumed to be the same across time but allowed to be different for different genes.

The shape parameter $\alpha$ is directly related to the $\mathrm{CV}$ through $\alpha=1 / \mathrm{CV}^{2}$ or $C V=1 / \sqrt{\alpha}$. The $C V$ is a unitless variation measure. The shape parameter accordingly can be thought of as a characterization for the stability or variability among the replicates sampled at a specific time under a treatment condition. Since, there is no biological evidence indicating the variability changes over time under the same treatment, we choose to assume it is constant across time. The shape parameter can also be interpreted as the inverse of the measure of variability among replicates at a specific time point. The shape parameter thus can be estimated by combining the variability information across time. The realized variability measure can only be obtained when the sample has two or more replicates at a specific time point. We use the estimated sample mean and sample variance to estimate the variability and treat the estimates at different time points as multiple realizations from the same underlying $\mathrm{CV}$ or shape parameter. In detail, for gene $g$ at time point $t$, the squared sample mean $\left(m_{g t}^{2}\right)$ over sample variance $\left(S_{g t}^{2}\right)$ can be used as the realized inversed variability measure $\hat{\alpha}_{g t}=\frac{m_{g t}^{2}}{S_{g t}^{2}}$ at time point $t$ for a specific gene $g$.

Using non-time series data, Lo and Gottardo (2007) showed the log-normal prior provides a good fit for the shape parameter. Non-time course data can be thought of as a special case with only one time point. The log-normal prior for time course data works well. The shape parameter is modeled by a log-normal distribution with mean parameter 
$\tau$ and variance parameter $\sigma$, i.e. $\alpha_{g t} \sim \log$-normal $(\tau, \sigma)$, where the log-normal distribution is defined as follows,

$$
p\left(\alpha_{g} \mid \tau, \sigma\right)=\frac{1}{\alpha_{g} \sigma \sqrt{2 \pi}} \exp \left(-\frac{\left(\log \left(\alpha_{g}\right)-\tau\right)^{2}}{2 \sigma^{2}}\right)
$$

As suggested by Lo and Gottardo (2007), the method of moment estimators for the two parameters in the lognormal prior are used here,

$$
\begin{gathered}
\hat{\tau}=\frac{\sum_{g=1}^{G} \sum_{t=1}^{T} \log \left(\hat{\alpha}_{g t}\right)}{G T} \\
\hat{\sigma}^{2}=\frac{\sum_{g=1}^{G} \sum_{t=1}^{T}\left(\log \left(\hat{\alpha}_{g t}\right)-\hat{\tau}\right)^{2}}{G T}
\end{gathered}
$$

After the hyper-parameters $(\tau, \sigma)$ are estimated from the data, the posterior distribution can be used to estimate the shape parameter for each gene. The posterior distribution for the shape parameter is as follows for the situation with $K$ distinct hidden states over $T$ time points with $R$ replicates at each time point:

$$
\begin{aligned}
L_{k}\left(\alpha \mid y_{11}, \ldots, y_{T R}, \underline{\mu}, \tau, \sigma\right)= & \frac{p\left(y_{11}, \ldots, y_{T R}, \underline{\mu} \mid \alpha\right) p(\alpha \mid \tau, \sigma)}{\int_{\alpha} p\left(y_{11}, \ldots, y_{T R}, \underline{\mu} \mid \alpha\right) p(\alpha \mid \tau, \sigma) d \alpha} \\
& \propto p\left(y_{11}, \ldots, y_{T R}, \underline{\mu} \mid \alpha\right) p(\alpha \mid \tau, \sigma)
\end{aligned}
$$

where $p\left(y_{11}, \ldots, y_{T R}, \mu_{1}, \ldots, \mu_{K} \mid \alpha\right)$ is the joint density of $y_{11}, \ldots, y_{T R}$ given $\alpha$, and $\underline{\mu}=\left(\mu_{1}, \ldots, \mu_{K}\right)^{T}$ 
The posterior without normalization for $\alpha$ conditional on $y$ is shown in (3.5). The same number of replicates $R$ at each time point is assumed. The total number of observations $n=R T$.

$$
\begin{aligned}
L_{k}\left(\alpha \mid y_{11}, \ldots, y_{T R}, \underline{\mu}, \tau, \sigma\right) \propto \Gamma^{-n}(\alpha) \alpha^{n \alpha} & \prod_{t=1}^{T} \prod_{r=1}^{R}\left\{y_{t r}^{\alpha-1} \sum_{k=1}^{K} \pi_{k} \mu_{k}^{-\alpha} \exp \left(\frac{-y_{t r} \alpha}{\mu_{k}}\right)\right\} \\
& \frac{1}{\alpha \sigma \sqrt{2 \pi}} \exp \left(\frac{-(\log (\alpha)-\tau)^{2}}{2 \sigma^{2}}\right)
\end{aligned}
$$

This un-normalized posterior requires $K$, the number of hidden states, which is unknown at this stage. The estimation of the shape parameter can not be performed based on (3.5) directly. This problem can be circumvented if the hidden states are defined according to the time points. As discussed in the first chapter, time series microarray experiments can be reasonably assumed to be synchronized so that at each time point the same stage of a biological process can be assumed. In other words, the expression intensities at each time point can be thought of as being generated from the same hidden state. At each specific time point $t$, the expressions are generated independently from a different $\operatorname{gamma}\left(\mu_{t}, \alpha\right)$ distribution. Assuming the distributions across time have different mean parameters, the distribution for the observations with $R$ replicates at the time point $t$ is:

$$
f_{t R}\left(y_{t 1}, y_{t 2}, \ldots, y_{t R} \mid \mu_{t}, \alpha\right)=\prod_{r=1}^{R} h\left(y_{t r} \mid \mu_{t}, \alpha\right)
$$

where $h\left(y_{t r} \mid \mu_{t}, \alpha\right)$ is the gamma distribution characterized by $\mu_{t}$ as defined in (2.2). Because we now know which distribution generates the observations, the complexity of 
(3.5) can be reduced immediately. This way the estimation of the shape parameter does not require information about the order $K$.

For each gene, the expressions at different time points are independent of each other given that the corresponding state is known, or equivalently, given the corresponding mean parameter $\mu_{t}$. Hence the distribution for all expressions over time for one gene is as follows:

$$
f_{T R}\left(y_{11}, \ldots, y_{T R} \mid \mu_{1}, \ldots, \mu_{T}, \alpha\right)=\prod_{t=1}^{T} \prod_{r=1}^{R} h\left(y_{t r} \mid \mu_{t}, \alpha\right)
$$

where $h\left(y_{t r} \mid \mu_{t}, \alpha\right)$ is the gamma distribution characterized by $\mu_{t}$ as defined in (2.2).

Based on (3.7), the un-normalized posterior function can be formulated as follows:

$$
\begin{aligned}
& L_{T R}\left(\alpha \mid y_{11}, \ldots, y_{T R}, \mu_{1}, \ldots, \mu_{T}, \tau, \sigma\right) \\
& \propto\left[\prod_{t=1}^{T} \prod_{r=1}^{R} h\left(y_{t r} \mid \mu_{t}, \alpha\right)\right] \frac{1}{\alpha \sigma \sqrt{2 \pi}} \exp \left(\frac{-(\log (\alpha)-\tau)^{2}}{2 \sigma^{2}}\right) \\
& \propto \Gamma^{-n}(\alpha) \alpha^{n \alpha}\left[\prod_{t=1}^{T} \prod_{r=1}^{R} y_{t r}^{\alpha-1} \mu_{t}^{-\alpha} \exp \left(-\frac{\alpha y_{t r}}{\mu_{t}}\right)\right] \frac{1}{\alpha \sigma \sqrt{2 \pi}} \exp \left(\frac{-(\log (\alpha)-\tau)^{2}}{2 \sigma^{2}}\right)
\end{aligned}
$$

This way, the shape parameter of each gene can be estimated without knowing the true number of hidden states. Above (3.8) can be thought of as a saturated model with the number of mean parameters larger than the true number of hidden states. In this sense, the order estimation conducted later is to decide which mean parameters are estimating the same true mean. Because the shape parameter is related to the variance, with mean parameter estimated separately for each time point, the fitting could be improved and more accuracy is expected for the variance estimate. Moreover, the observed variability (i.e. the realized shape parameter value) can be obtained from a sample at a specific time 
point. Combining two samples together has the risk that they may come from different states. There is no risk if we do not combine two samples together when they actually come from the same population. On the other hand, combining any of the samples may introduce bias because whether those samples combined come from the same distribution or not is not known at this point. Estimating the shape parameter independent of the order and treating the distribution at different time points to be different can make the estimation of shape parameter more robust to potential error in the order estimation.

The sample mean, which is the MLE of the mean parameter for the gamma distribution, is used in (3.8) to estimate $\mu_{t}$. Since microarray analysis typically involves a large number of parameters to be estimated, it is a common to estimate some parameters based on the estimated value of other parameters. Under the condition that the distributions are all different in terms of mean across time, the information about the mean can be only obtained based from the data at the corresponding time point.

With all other parameters estimated, the shape parameters can be estimated. Since the estimation for the shape parameter needs to be performed for each time one at a time, to reduce computational burden, the mode of the un-normalized posterior is chosen for the estimator. Since there is no closed form solution for the mode of (3.8), we use numeric methods to find the mode for each gene. Of course, the mean, median or mode of the posterior can be considered in the estimation. We chose the mode here mainly due to computational considerations. 
The difference between a typical mixture model specification and above distribution in (3.7) is that the allocation identity for each observation is known in the latter situation. In other words, for each data point, we know exactly which state it belongs to when the state is defined by time point. Hence, it is no longer a mixture model. The estimation of the shape parameter is accordingly reduced to the situation where we have several samples taken over time and we estimate the shape parameter by combining the information of multiple samples together. 


\section{Chapter 4}

\section{ORDER ESTIMATION}

The problem of estimating the number of hidden states (referred to as the order estimation problem) has been widely discussed in both the finite mixture model and hidden Markov model literature. Although there are quite a few approaches proposed, this problem has not been satisfactorily solved yet. In this chapter, we first review current literature for order estimation in both finite mixture models and HMMs in section 4.1. We then discuss our proposed method with a focus on the microarray data, in section 4.2. Next we discuss related issues in subsequent sections. The computation algorithm is discussed in section 4.3 and the choice of threshold values is discussed in section 4.4 . The identifiability issue is briefly discussed in section 4.5 .

\subsection{Literature Review of Order Estimation for HMMs and Finite}

\section{Mixture Models}


The order of a hidden Markov model can be defined as the number of distinct hidden states. Or as suggested by Cappe et al. (2004), the order of a HMM is the minimum size of the hidden state space of an HMM that can generate the observations. Estimation of the parameters of an HMM when the true order is known has been extensively studied. The consistency and asymptotic normality of the maximum likelihood estimates were established by Leroux (1992) and Bickel et al. (1998) respectively. As discussed above, the stationary probability is commonly assumed in estimating the order for HMMs, making the order estimation of HMMs the same as that of finite mixture models.

The problem of estimating the order of HMMs or finite mixture models has not been fully solved yet. The Akaike information criterion (AIC) and Bayes information criterion (BIC) approaches were adapted as proposed estimation methods by Leroux and Puterman (1992), Hughes and Cuttorp (1994), Albert et al. (1994) and Wang and Puterman (1999). These applications of AIC or BIC criteria did not establish that statistical properties such as consistency held (MacDonald and Zucchini, 1997). One important approach for order estimation is penalized likelihood methods. Baras and Finesso (1992) developed a consistent estimator of the order when the observations are discrete. Leroux and Puterman (1992) and Ryden (1995) analyzed the problem using model selection techniques. Ryden (1995) established that information criteria such as AIC and BIC do not under-estimate the true number of states asymptotically. Csiszar and Shields (2000) investigated the asymptotical property for both over-estimation and underestimation. They established the consistency of BIC for order estimation. Following this 
path, MacKay (2002), and Poskitt and Zhang (2005) proposed two new estimators based on penalized maximum likelihood methods. They established the consistency property for penalized likelihood estimators. In addition to penalized maximum likelihood estimation of the order of HMMs, likelihood ratio testing is another option to find certain information about the order. Hansen (1992) and Hamilton (1996) provided initial studies of this problem. Hamilton (1996) also provided several tests of model misspecification. Heckman, Robb and Walker (1990) developed a test statistic based on the method of moments. Feng and McCulloch (1996) proposed the likelihood ratio statistic. Chen, Chen and Kalbfleisch (2001) also provided a modified likelihood ratio test for homogeneity in finite mixture models.

Poskitt and Zhang (2005) further argued that order estimation of HMMs can be based on the marginal distribution based on a stationary HMM. Order estimation of HMMs in this way is the same problem as the order estimation of finite mixture models. In finite mixture model situations, the order is defined as the number of unique mixture component distributions.

For finite mixture models, the number of distinct component distributions is also referred to as the order of the mixture model. Hereafter, the order is referred to as the order of both finite mixture models and the stationary HMMs. The problem of order estimation has not been solved in finite mixture models either (McLachlan and Peel, 2000). Roeder and Wasserman (1997) have shown that when a normal mixture model is used to estimate a density, the density estimate that uses BIC to select the number of components in the mixture is consistent. Other proponents for the use of AIC or BIC in 
this situation are discussed in Biernacki et al. (1998), Cwik and Koronacki (1997) and Solka et al. (1998). Richardson and Green (1997) also provided a Bayesian treatment of the order estimation problem.

As suggested by MacLachlan and Peel (2000), "it is therefore sensible in practice to approach the question of the number of components in a mixture model in terms of an assessment of the smallest number of components in the mixture compatible with the data." And the order can be defined to be the smallest value of $K$ in (2.1) such that all the components $h_{k}\left(y_{t r} \mid \mu_{k}, \alpha\right)$ are different and all the associated mixing proportions $\pi_{k}$ are nonzero. These two conditions require the components to be distinct. They are also important to maintain the model's identifiability as we will discuss below.

In this thesis, we develop our method based on the penalized maximum likelihood estimation approach. The difficulty of order estimation in stationary HMMs (or equivalently order estimation for finite mixture models) mainly comes from the monotonic increase of the likelihood as the order increases. The methods developed by Chen and Kalbfleisch (1996) and James et al. (2001) try to minimize the distance between a nonparametric curve based on the sample of data and the fitted model. Poskitt and Zhang (2005) proposed to use a penalized quasi-likelihood estimator and investigated its asymptotic properties. Here we adopt the penalized maximum likelihood method with two penalty terms proposed in Chen and Khalili (2005). The consistency of the penalized maximum likelihood estimators are established by Chen and Khalili (2005) and Poskitt and Zhang (2005). Our focus here is on the small sample situation as it is quite typical for microarray time course data. 
There are two penalty terms in our proposed penalized likelihood. The second penalty term was first proposed by Chen and Kalbfleisch (1996) and was also used by MacKay (2002) in estimating the order of HMMs. The first penalty term is based on the development of the least absolute shrinkage and selection operator (LASSO) function (Tibshirani, 1996) and the smoothly clipped absolute deviation (SCAD) function (Fan and $\mathrm{Li}, 2001)$. We extend this two-term penalized likelihood method and propose a measure of the distance between adjacent emission distributions for the first penalty term, which we shall refer to as separation probability. This way, we have a probabilistic measure of how different adjacent components are and therefore provide a way to compare the distance between multiple adjacent component distributions. Chen and Khalili (2005) developed the two-term penalized maximum likelihood method to estimate the number of mixture components when the variance of normal component distributions are known and equal to 1, while the mean is the only unknown parameter. Our method extends theirs to the situation where the mean of gamma mixture distributions is unknown, while the variances are different and also unknown.

\subsection{Order Estimation}

According to Chen and Khalili (2005), an upper bound for the order, denoted as $K_{u}$, needs to be specified at the starting point. It is required to be at least as larger as the true order. For microarray time course analysis, we propose to start with the upper bound $K_{u}$ as 5 . This is because an order that is too large requires that the means of the mixture 
components be spread out in a wide range which is probably not the case for microarray data. For a reasonable separation probability, starting with an upper bound of five is usually sufficient for microarray data. Because the biological evidence regarding possible different responses is mostly between binding and non-binding, it does not suggest a large number of states. Although starting with a higher upper bound with data having more time points and replicates is possible, an upper bound of 5 can be a reasonable starting point based on our analysis.

The log-likelihood function of the stationary hidden Markov model (or equivalently the mixture models) based on gamma mixture components with $K$ hidden states for gene $g$ is defined as follows, where $f_{K}\left(y_{t r} \mid \underline{\pi}, \underline{\mu}, \alpha\right)$ is defined as in (2.1),

$$
\begin{array}{r}
\underline{\pi}=\left(\pi_{1}, \ldots, \pi_{K}\right)^{T} \text { with } \sum_{1}^{K} \pi_{k}=1, \text { and } \underline{\mu}=\left(\mu_{1}, \ldots, \mu_{K}\right)^{T} . \\
l_{K}(K, \underline{\pi}, \underline{\mu}, \alpha)=\sum_{t=1}^{T} \sum_{r=1}^{R_{t}} \log \left(f_{K}\left(y_{t r} \mid \underline{\pi}, \underline{\mu}, \alpha\right)\right)
\end{array}
$$

To prevent two types of over-fitting, two penalty terms are included in the following penalized log-likelihood:

$$
\tilde{l}_{K}(K, \underline{\pi}, \underline{\mu}, \alpha)=l_{K}(K, \underline{\pi}, \underline{\mu}, \alpha)-\sum_{k=1}^{K-1} p\left(\eta_{k}\right)+C_{k} \sum_{k=1}^{K} \log \pi_{k}
$$

The function $p\left(\eta_{k}\right)$ is a non-decreasing function of $\eta_{k}$ on $(0,+\infty)$ and $p(0)=0$. It is twice differentiable for $\eta_{k}$ except for a finite number of points. The first penalty term is used to penalize the likelihood if the means of some mixture components are too 
close to each other. It is designed so that if any $\eta_{k}$ has a small fitted value without the first penalty term, its fitted value with the first penalty term has a positive chance to be zero. Note that the mean parameter is the only parameter depending on the hidden state at corresponding time point. The second penalty term, originally developed by Chen and Kalbfleisch (1996) is used to penalize the likelihood for small values of mixing proportions. $C_{k}$ is a constant. For a given upper bound $K_{u}$, the means of component distributions first need to be arranged in increasing order as $\mu_{(1)}<\mu_{(2)}<\mu_{(3)}, \ldots \ldots ., \mu_{\left(K_{u}\right)}$ and define the distance measure for adjacent means as:

$$
\eta_{k}=\mu_{(k+1)} / \mu_{(k)}-1
$$

for some $K_{u} \geq 2$.

The first penalty is calculated based on this distance between two adjacent means of mixture components using a penalty function called the hard penalty function. It is defined as follows. The $\lambda$ is the threshold for $\eta$.

$$
p(\eta)=\lambda^{2}-(|\eta|-\lambda)^{2} I(|\eta| \leq \lambda)
$$

Fan and $\mathrm{Li}(2001,2002)$ discussed other possible penalty functions and provided detailed comparisons of their performance. We choose to use the hard penalty term mainly for computational considerations since the algorithm needs to run for each gene one at a time in a microarray.

In the computational implementation, a simplified procedure can be used as proposed by Fan and $\mathrm{Li}(2001,2002)$. The procedure is: when the penalized value is 
smaller than the threshold, simply set the value to zero. This is based on the observation that once the penalized value falls under a threshold value, the penalized likelihood function will never produce a value larger than the threshold; when the penalized value is larger than a threshold, an approximation function can be used to derive the updated parameter values for different penalty functions. This simplified procedure actually unifies the implementation of different penalty functions. As defined above $\eta_{k}=\mu_{(k+1)} / \mu_{(k)}-1, \eta_{k}$ is the parameter used in the hard penalty term. When $\eta_{k}$ is smaller than a threshold value, it will be set to zero by our algorithm. This is equivalent to setting $\mu_{(k+1)}=\mu_{(k)}$, which leads to fewer distinct mean parameters, and accordingly the order estimated is reduced by 1 .

For a given threshold $\lambda$ in the hard penalty term, the penalized log-likelihood (4.2.2) is reduced by a constant $\lambda^{2}$ when $\eta>\lambda$. Because of the constant, the penalized MLE does not produce additional bias, compared to the original MLE. On the other hand, when $\eta \leq \lambda$, the first penalty term is smaller (and hence the likelihood is larger) and the penalty value becomes smaller as $\eta$ tends to zero.

Note that inference about $K$ is conducted indirectly. The likelihood function and the hard penalty function do not include $K$. Instead, the $K$ is indirectly inferred based on the information about all mean parameters. Because the order is defined as the number of distinct hidden states or equivalently the number of distinct mean parameters, those means with the same value will be counted only once in the final step for the estimated order. The hard penalty term does not directly work on the hidden states either. Instead, it looks at the distance or difference between two adjacent means. Once they move closer to 
each other than a threshold, the two corresponding states will be regarded as the same. And $K$ will be reduced by 1 . In this sense, our procedure is to merge those means once they are closer to each other below a specified threshold. Because this is an indirect approach, the likelihood (4.2.2) does not need to include hidden states or the order $K$, making the likelihood much simpler and thus more suitable for small sample situations such as microarray data.

Starting from the upper bound $K_{u}=5$, our algorithm first sets initial mean parameter values to be spread over the range of observed expressions. With each iteration, the estimates of mean parameters change their values to increase the likelihood. Our algorithm then sets those mean estimates equal when they are close to each other and sets the mixing proportions to zero when they are sufficiently small. Because the estimated order is the number of unique mean values, setting two means equal is equivalent to reducing the order by 1 . The condition $\sum_{1}^{K} \pi_{k}=1$ is also maintained in each iteration.

We further propose to use separation probability $p_{\eta}$ as an alternative distance measure between two adjacent means, in addition to $\eta$. Accordingly a separation threshold, $p_{\lambda}$, can be specified in the same way as choosing a $\lambda$ value for $\eta$. Expressing the distance of two adjacent means in a probability measure may help us to understand how strong a biological signal in terms of probability measure. On the other hand, these two distance measures have the same function in the penalty term. Therefore either one can be used. 
Our model setting allows the shape parameter to be common for all the gamma mixture components while the means differ across time. The distance between two adjacent means can be defined as in (4.2.3) which takes into account the possible different variances involved for different pair-wise distances. For ease of discussion we assume that the means of the mixture components are always arranged in ascending order hereafter. Because distances of all pairs of adjacent means need to be compared with a pre-determined threshold $\lambda$ value, these distances obtained for different pairs should be comparable. As we allow the variance of mixture components to be different, the variances of two adjacent mixture components can potentially affect the distance of means. Therefore the distance needs to be adjusted for the variance effect across different adjacent pairs. Hence, we define the distance between two adjacent means as the ratio of adjacent means minus 1 , or equivalently, as the difference between two adjacent means divided (or standardized) by the smaller mean. A threshold $\lambda$ value then can be specified for the standardized distance between all adjacent pairs.

The above standardized distance across different adjacent pairs can be expressed in our proposed separation probability measure. We will show that the standardized distance measure results in the same separation probability across different adjacent pairs for gamma distributions. We explain this with an example of three gamma mixture components. Suppose there are three mixture component distributions with common shape parameter $\alpha$ but different mean parameters in increasing order as $\mu_{(1)}<\mu_{(2)}<\mu_{(3)}$ with the corresponding cumulative distribution functions $F_{(1)}, F_{(2)}, F_{(3)}$. The separation probability between $F_{(1)}$ and $F_{(2)}$ is defined as $F_{(1)}\left(x_{0}\right)$, where $x_{0}$ is the value that maximizes $F_{(1)}-F_{(2)}$, with $x_{0} \in R^{+}$for the gamma distribution. As defined in 
this way, if the separation probability between the first and second mixture component is the same as the separation probability between the second and third distribution, the ratio of two adjacent means is a constant; that is $\frac{\mu_{(2)}}{\mu_{(1)}}=\frac{\mu_{(3)}}{\mu_{(2)}}$ and vise versa. The separation threshold has a one-to-one relationship with the threshold of the distance measure $\lambda$ for a given shape parameter. Note that the relationship may not be valid when the shape parameter is not the same across component distributions.

Defined this way, the separation probability can be interpreted biologically as follows. For a given separation probability $s$ between two states characterized by distributions $F_{(1)}, F_{(2)}$ for gene A, there is a probability of $1-s$, that the first state is mistakenly understood to be the second state by those regulated genes of gene A.

Here we prove that the separation probability between the first and second mixture component is the same as the separation probability between the second and third distribution if and only if the ratio of two adjacent mean parameters or the ratio of the two scale parameter is constant $\frac{\mu_{2}}{\mu_{1}}=\frac{\mu_{3}}{\mu_{2}}=\frac{\beta_{2}}{\beta_{1}}=\frac{\beta_{3}}{\beta_{2}}=$ Const., where $\mu_{1}, \mu_{2}$ and $\mu_{3}$ are the mean parameter for the gamma distribution functions $F_{(1)}, F_{(2)}$ and $F_{(3)}$ respectively and $\beta_{1}, \beta_{2}$ and $\beta_{3}$ are the scale parameters for the gamma distribution functions $F_{(1)}, F_{(2)}$ and $F_{(3)}$ respectively. 


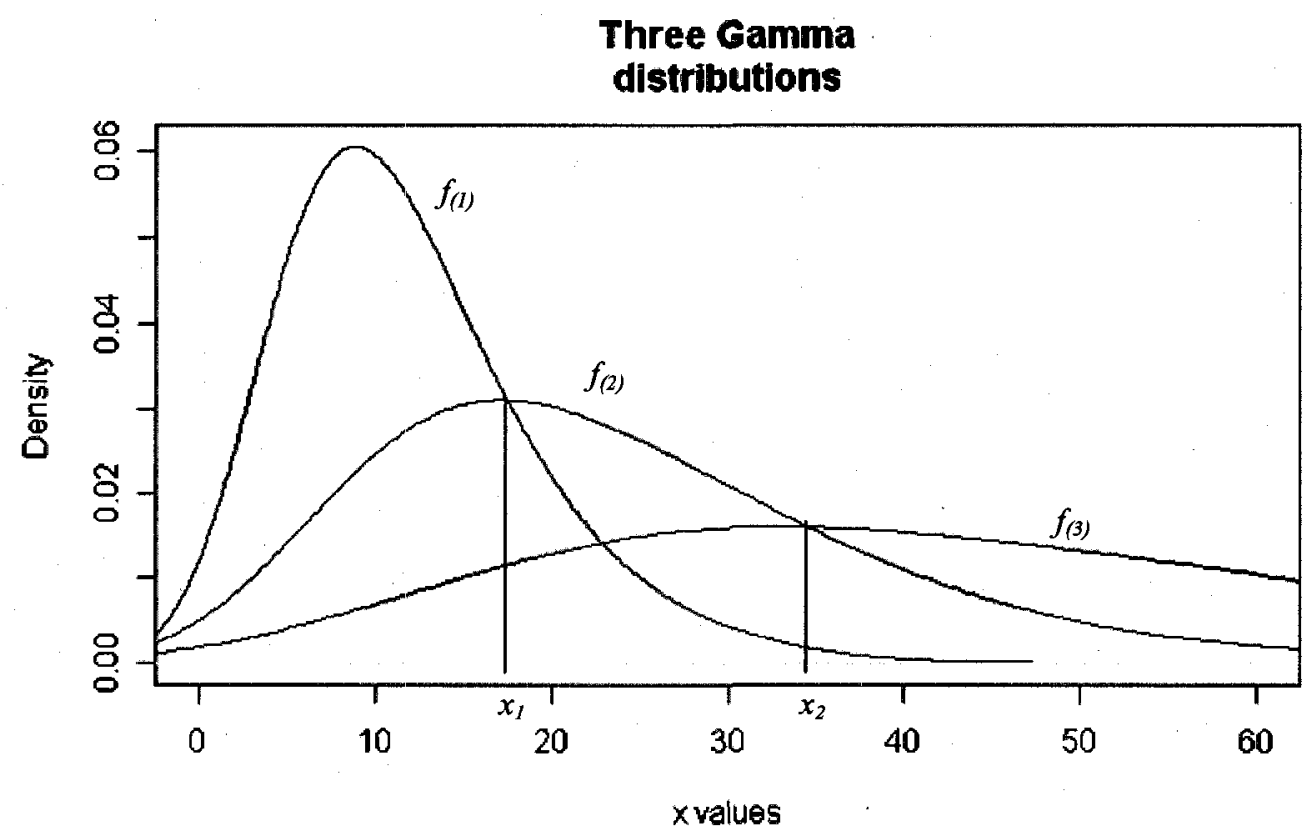

Figure 4.2.1. A gamma mixture of three component distributions, with probability density functions $f_{(1)}, f_{(2)}$ and $f_{(3)}$.

Figure 4.2.1 presents three cumulative distribution functions $F_{(1)}, F_{(2)}$ and $F_{(3)}$ with increasing mean values but the same shape parameter. Corresponding to the cumulative distribution functions $F_{(1)}, F_{(2)}$ and $F_{(3)}$ are the density functions $f_{(1)}, f_{(2)}$ and $f_{(3)}$. At $x_{1}, f_{(1)}\left(x_{1}\right)=f_{(2)}\left(x_{1}\right)$ and at $x_{2}, f_{(2)}\left(x_{2}\right)=f_{(3)}\left(x_{2}\right)$.

We first show that at $x_{1}, F_{(1)}-F_{(2)}$ is maximized. That is, $x_{1}$ is the point at which $F_{(1)}\left(x_{1}\right)$ defines the separation probability between $F_{(1)}$ and $F_{(2)}$. To find the point where the function $F_{(1)}-F_{(2)}$ is maximized, we need to take the derivative with respect to $x$ and let it equal to zero.

$$
\frac{d}{d x}\left(F_{(1)}(x)-F_{(2)}(x)\right)=0
$$


which is:

$$
\frac{d}{d x}\left(F_{(1)}(x)-F_{(2)}(x)\right)=\frac{d}{d x}\left(\int_{0}^{x} f_{(1)}(z) d z-\int_{0}^{x} f_{(2)}(z) d z\right)=\frac{d}{d x} \int_{0}^{x} f_{(1)}(z) d z-\frac{d}{d x} \int_{0}^{x} f_{(2)}(z) d z=0
$$

thus:

$$
f_{(1)}(x)=f_{(2)}(x)
$$

We denote this point as $x_{I}$ as shown in Figure 4.2.1. Therefore according to the definition of separation probability above, the separation probability between $F_{(1)}$ and $F_{(2)}$ is $F_{(1)}\left(x_{1}\right)$. Similarly, the separation probability between $F_{(2)}$ and $F_{(3)}$ is $F_{(2)}\left(x_{2}\right)$.

Next, we would like to show that if $\frac{\mu_{2}}{\mu_{1}}=\frac{\mu_{3}}{\mu_{2}}=\frac{\beta_{2}}{\beta_{1}}=\frac{\beta_{3}}{\beta_{2}}=a$, where $a$ is an arbitrary constant, then the separation probability between $F_{(1)}$ and $F_{(2)}$ and $F_{(2)}$ and $F_{(3)}$ is the same; that is, $F_{(1)}\left(x_{1}\right)=F_{(2)}\left(x_{2}\right)$. As proved above: $f_{(1)}\left(x_{1}\right)=f_{(2)}\left(x_{1}\right)$ and $f_{(2)}\left(x_{2}\right)=$

$f_{(3)}\left(x_{2}\right)$. And $\frac{\mu_{2}}{\mu_{1}}=\frac{\mu_{3}}{\mu_{2}}=\frac{\beta_{2}}{\beta_{1}}=\frac{\beta_{3}}{\beta_{2}}=a$ indicates $\beta_{2}=a \beta_{1}, \beta_{3}=a \beta_{2}=a^{2} \beta_{1}$.

Therefore we have

$$
\begin{aligned}
& \frac{1}{\Gamma(\alpha) \beta_{1}^{\alpha}} x_{1}^{\alpha-1} \exp \left(\frac{-x_{1}}{\beta_{1}}\right)=\frac{1}{\Gamma(\alpha)\left(a \beta_{1}\right)^{\alpha}} x_{1}^{\alpha-1} \exp \left(\frac{-x_{1}}{a \beta_{1}}\right) \\
& \frac{1}{\Gamma(\alpha)\left(a \beta_{1}\right)^{\alpha}} x_{2}^{\alpha-1} \exp \left(\frac{-x_{2}}{a \beta_{1}}\right)=\frac{1}{\Gamma(\alpha)\left(a^{2} \beta_{1}\right)^{\alpha}} x_{2}^{\alpha-1} \exp \left(\frac{-x_{2}}{a^{2} \beta_{1}}\right)
\end{aligned}
$$

Simplifying the above two equations, we then have 


$$
\begin{aligned}
& \beta_{1}^{-\alpha} \exp \left(\frac{-x_{1}}{\beta_{1}}\right)=\left(a \beta_{1}\right)^{-\alpha} \exp \left(\frac{-x_{1}}{a \beta_{1}}\right) \\
& \left(a \beta_{1}\right)^{-\alpha} \exp \left(\frac{-x_{2}}{a \beta_{1}}\right)=\left(a^{2} \beta_{1}\right)^{-\alpha} \exp \left(\frac{-x_{2}}{a^{2} \beta_{1}}\right)
\end{aligned}
$$

By dividing each side of (4.2.10) by the corresponding side of (4.2.11), the following can be obtained:

$$
\left(\frac{1}{a}\right)^{-\alpha} \exp \left(\frac{-x_{1}}{\beta_{1}}+\frac{x_{2}}{a \beta_{1}}\right)=\left(\frac{1}{a}\right)^{-\alpha} \exp \left(\frac{-x_{1}}{a \beta_{1}}+\frac{x_{2}}{a^{2} \beta_{1}}\right)
$$

Simplifying the above, we get $x_{2}=a x_{1}$.

For a gamma distribution, it is true that if $X \sim \operatorname{gamma}\left(\alpha, \beta_{1}\right)$, then $a X \sim \operatorname{gamma}\left(\alpha, a \beta_{1}\right)$ or equivalently, $a X \sim \operatorname{gamma}\left(\alpha, \beta_{2}\right)$ where $\beta_{2}=a \beta_{1}$. At $x_{1}$, the separation probability is defined as $F_{(l)}\left(x_{l}\right)=P_{(I)}\left(X<x_{l}\right)$. Then we have:

$$
F_{(1)}\left(x_{1}\right)=P_{(1)}\left(X<x_{1}\right)=P_{(1)}\left(a X<a x_{1}\right)=P_{(1)}\left(a X<x_{2}\right)=P_{(2)}\left(a X<x_{2}\right)=F_{(2)}\left(x_{2}\right)
$$

This means the separation probability between $F_{(1)}$ and $F_{(2)}$ and between $F_{(2)}$ and $F_{(3)}$ is the same.

Next, we prove that if $F_{(1)}\left(x_{1}\right)=F_{(2)}\left(x_{2}\right)=p$, where $p$ is a given arbitrary positive constant, then $\frac{\mu_{2}}{\mu_{1}}=\frac{\mu_{3}}{\mu_{2}}=\frac{\beta_{2}}{\beta_{1}}=\frac{\beta_{3}}{\beta_{2}}$.

For a given separation probability $p$, we have $F_{(1)}\left(x_{1}\right)=P_{(1)}\left(X<x_{1}\right)=F_{(2)}\left(x_{2}\right)$ $=P_{(2)}\left(Y<x_{2}\right)=p$. As the shape parameter $\alpha$ is the same for the three distributions, we can 
assume the density functions for $f_{(2)}, f_{(3)}$ have the form $f_{(2)}=\operatorname{gamma}(\alpha, h \beta)$ and $f_{(3)}=\operatorname{gamma}(\alpha, k \beta)$. We want to show $h=k / h$, i.e. $k=h^{2}$ if $F_{(1)}\left(x_{1}\right)=F_{(2)}\left(x_{2}\right)=p$ is true.

As proved above, $f_{(1)}\left(x_{1}\right)=f_{(2)}\left(x_{1}\right)$ and $f_{(2)}\left(x_{2}\right)=f_{(3)}\left(x_{2}\right)$. Then, the following two equations can be obtained:

$$
\begin{aligned}
& \frac{1}{\Gamma(\alpha) \beta^{\alpha}} x_{1}^{\alpha-1} \exp \left(\frac{-x_{1}}{\beta}\right)=\frac{1}{\Gamma(\alpha)(h \beta)^{\alpha}} x_{1}^{\alpha-1} \exp \left(\frac{-x_{1}}{h \beta}\right) \\
& \frac{1}{\Gamma(\alpha)(h \beta)^{\alpha}} x_{2}^{\alpha-1} \exp \left(\frac{-x_{2}}{h \beta}\right)=\frac{1}{\Gamma(\alpha)(k \beta)^{\alpha}} x_{2}^{\alpha-1} \exp \left(\frac{-x_{2}}{k \beta}\right)
\end{aligned}
$$

Simplifying the above two equations, we then have

$$
\begin{aligned}
& \beta^{-\alpha} \exp \left(\frac{-x_{1}}{\beta}\right)=(h \beta)^{-\alpha} \exp \left(\frac{-x_{1}}{(h \beta)}\right) \\
& (h \beta)^{-\alpha} \exp \left(\frac{-x_{2}}{h \beta}\right)=(k \beta)^{-\alpha} \exp \left(\frac{-x_{2}}{k \beta}\right)
\end{aligned}
$$

Dividing each side of (4.2. 16) by the corresponding side of (4.2.17), the following can be obtained:

$$
\left(\frac{1}{h}\right)^{-\alpha} \exp \left(\frac{-x_{1}}{\beta}+\frac{x_{2}}{h \beta}\right)=\left(\frac{h}{k}\right)^{-\alpha} \exp \left(\frac{-x_{1}}{h \beta}+\frac{x_{2}}{k \beta}\right)
$$

Based on the function we assumed above for $f_{(2)}$ and $f_{(3)}$, which is $f_{(2)}=\operatorname{gamma}(\alpha, h \beta)$ and $f_{(3)}=\operatorname{gamma}(\alpha, k \beta)$, we know that if $X \sim f_{(1)}(x): \operatorname{gamma}(\alpha, \beta)$, then let $Y=h X$, and $Y \sim f_{(2)}(y): \operatorname{gamma}(\alpha, h \beta)$. Because $P\left(Y<x_{2}\right)=P\left(h X<x_{2}\right)$ (according to 
the relationship between $X$ and $Y$ we just defined); while $P\left(h X<x_{2}\right)=P\left(X<\left(x_{2} / h\right)\right)$ (use the probability function of $X) ; P\left(Y<x_{2}\right)=P\left(X<\left(x_{2} / h\right)\right)=P\left(X<x_{1}\right)$ (use the condition we assumed at the beginning); and $P(X)=F_{(I)}(x)$ is a monotonic increasing function. Therefore $x_{2}=h x_{1}$. Then (4.2.19) becomes:

$$
\left(\frac{1}{h}\right)^{-\alpha} \exp \left(\frac{-x_{1}}{\beta}+\frac{h x_{1}}{h \beta}\right)=\left(\frac{h}{k}\right)^{-\alpha} \exp \left(\frac{-x_{1}}{h \beta}+\frac{h x_{1}}{k \beta}\right)
$$

And further we can obtain:

$\left(\frac{1}{h}\right)^{-\alpha}=\left(\frac{h}{k}\right)^{-\alpha} \exp \left(\frac{-x_{1}}{h \beta}+\frac{h x_{1}}{k \beta}\right)$, for arbitrary positive values of $\alpha, \beta, x_{1}$. The solution leads to $k=h^{2}$. That is, the ratio of two adjacent mean parameters or scale parameters is constant.

The relationship between the separation probability and the standardized distance between adjacent means, as we shown above, is closely related. Their function in the first penalty is the same. They provide a measure which makes the distances between different pairs of adjacent component distributions comparable. The threshold value, either the standardized distance threshold $\eta$ or the separation threshold $p_{\eta}$, can be chosen in a similar way. Actually in our model setting, they have a one-to-one relationship.

Our order estimation method is developed with two considerations in mind. The first is our primary goal to analyze microarray time course data. The typical limited sample size and limited time points make it very difficult to estimate the order precisely. These limitations are well understood by the biological community and hence microarray 
analysis is used as a screening tool to help to narrow down the candidate genes to a small subset of genes. These genes may need to be further examined using other experimental techniques. Our method is developed mainly to address this objective. We have designed a type I error rate based approach to specify the threshold value for the first penalty term so that the threshold can be determined by simulated data. Of course our simulation is based on the information of sample size, the shape parameter values and the desired type I error rate level for a specific microarray data set. Details will be discussed in section 4.4. The advantage of this approach is that it does not require pre-knowledge of the separation probability so that the microarray time course can be immediately analyzed. Another advantage is that we can control the accuracy to divide the genes into a response set with order as one and another set with order two or above. The disadvantage is that the accuracy of order estimation among those genes with order above 2 in the response set can not be controlled. However, we believe that identifying those response genes with order as two or above is sufficient to serve the screening function in microarray analysis. In addition, our method provides a way to characterize the type I error rate so that the accuracy can be measured and controlled. We suggest that further order estimation for the multiple order genes needs to be performed with more detailed experimental measures rather than the limited data from a microarray. With microarray data alone, with such a limited sample size and no pre-knowledge of separation probability, it is not realistic to expect the higher order can be quantified reliably.

The secondary goal of our method is to provide some methodological discussion for the situation where a sufficiently large sample is available. The large samples may be obtained by more detailed measurements of the response genes identified with our 
method. Given a sufficiently large sample size, the threshold for the first penalty can be obtained by two data driven methods as discussed by Chen and Khalili (2005). Note that the estimation of the exact order requires pre-knowledge of the threshold values for the hard penalty term. This knowledge certainly is not available for microarray data because microarray experiments are typically conducted before other experiments. Since microarray analysis is our primary goal in this thesis, we focus on the discussion of order estimation for small sample situations. We will only outline the key direction for exact order estimation for medium to large sample sizes without going into detail.

\subsection{Computational Algorithm}

The algorithm for the above computation is a version of the EM algorithm (Dempster et al., 1977) and can be summarized in the following steps. There is some revision of the $M$ step.

1. Specify initial values for the upper bound of order, mean parameters and stationary probabilities. Specify the separation threshold value for the first penalty term and the constant for the second penalty term.

2. E step: Calculate the conditional expectation as shown in (4.3.1) for (4.2.2) conditioning on the current estimated parameter values.

3. M step: Update the stationary probability by maximizing the conditional expectation function in (4.3.1). Update the mean parameters by maximizing (4.3.1). If the separation 
probability is less than the specified threshold, merge the two adjacent means so that they have the same estimated mean in all subsequent iterations. Go to step 2.

4. The iteration between step 2 and step 3 stops when the increase of the penalized likelihood is sufficiently small. Alternatively, a sufficiently large number of iterations can be used.

The initial values of the mean parameters and the initial values of the mixing proportions need to be first specified. Our simulation shows that these initial values are not critical as long as the means are not too close to one another or concentrate in a small range. An equal mixing proportion based on an upper bound of the order can be chosen at the start. We normally choose the initial means such that they spread out equally within the range of expression levels.

In detail, the EM algorithm maximizes (4.2.2) iteratively in two steps as follows. E step: Let $\Psi^{(m)}$ be the estimate of the parameters after the $m$ th iteration. The E step first calculates the conditional expectation of (4.2.2) given the observed data and the parameter estimates from the previous iteration. For a particular gene with a total of $n$ observations, using the standardized distance $\eta_{k}=\frac{\mu_{k+1}}{\mu_{k 1}}-1=\frac{\beta_{k+1}}{\beta_{k 1}}-1$ as an example, the conditional expectation is

$$
\begin{aligned}
Q\left(\Psi ; \Psi^{(m)}\right)=\sum_{i=1}^{n} \sum_{k=1}^{K} w_{i k}^{(m)} & \log \left\{f\left(y_{i} \mid \beta_{k}\right)\right\}-\sum_{k=1}^{K-1} p\left(\eta_{k}\right) \\
& +\sum_{i=1}^{n} \sum_{k=1}^{K}\left\{w_{i k}^{(m)}+\frac{C_{k}}{n}\right\} \log \pi_{k}
\end{aligned}
$$

where 


$$
w_{i k}^{(m)}=\frac{\pi_{k}^{(m)} f\left(y_{i} \mid \beta_{k}^{(m)}\right)}{\sum_{l=1}^{K} \pi_{l}^{(m)} f\left(y_{i} \mid \beta_{l}^{(m)}\right)}, k=1,2, \ldots \ldots, K
$$

is the conditional expectation of the probability of each observation belonging to a mixture component given the observed data and the current estimated parameters. The gamma distribution for each component distribution is parameterized as $\operatorname{gamma}\left(\alpha, \beta_{k}\right)$ for the corresponding $\operatorname{gamma}\left(\alpha, \mu_{k}\right)$, with $\mu_{k}=\alpha \beta_{k}$. The parameter $\alpha$ is treated as known for each gene based on the estimation performed in Chapter 3. (4.3.1) is the conditional expectation of (4.2.2). The values of mean parameters (or equivalently the values of scale parameters) determine the hidden states. The order can be obtained by counting the distinct mean or scale parameters once the iteration is completed. The same procedure is used in Chen and Khalili (2005) to estimate the number of mixture components.

M step: update the parameter values for $(m+1)$ iteration by maximizing $Q\left(\Psi \mid \Psi^{(m)}\right)$ in (4.3.1). First $\pi_{k}^{(m+1)}$ is updated by maximizing the expectation with respect to the mixing proportions $\pi_{k}$. The derived new estimates are:

$$
\pi_{k}^{(m+1)}=\frac{\sum_{i=1}^{n} w_{i k}^{(m)}+C_{K}}{n+K C_{K}}, k=1,2, \ldots \ldots, K
$$

(4.3.3) is the result of the following maximization steps:

1. Maximize $Q\left(\Psi \mid \Psi^{(m)}\right)$ in (4.3.1) with respect to $\pi_{k}$, which is the same as maximizing $\sum_{i=1}^{n} \sum_{k=1}^{K}\left\{w_{i k}^{(m)}+\frac{C_{k}}{n}\right\} \log \pi_{k}$ with respect to $\pi_{k}$. 
2. Under the constraint that $\sum_{k=1}^{K} \pi_{k}=1$, the Lagrange multiplier technique is used. Set

$$
\mathrm{A}=\sum_{i=1}^{n} \sum_{k=1}^{K}\left\{w_{i k}^{(m)}+\frac{C_{k}}{n}\right\} \log \pi_{k}+\delta\left(\sum_{k=1}^{K} \pi_{k}-1\right)
$$

where $\delta$ is a constant.

3. Let $\frac{\partial A}{\partial \pi_{k}}=0$ for $k=1,2, \ldots, K$. We will get $\mathrm{K}$ equations. As an example for $k$ th equation we have:

$$
\frac{\partial A}{\partial \pi_{k}}=\sum_{i=1}^{n}\left(w_{i k}^{(m)}+\frac{C_{K}}{n}\right) \frac{1}{\pi_{k}}+\delta=\left(n \pi_{k}+\frac{C_{K}}{n}\right) \frac{1}{\pi_{k}}+\delta=n+\frac{C_{K}}{\pi_{k}}+\delta=0
$$

so that we have:

$$
n \pi_{k}+C_{K}+\delta \pi_{k}=0
$$

4. Add above (4.3.6) for $k=1, \ldots, K$ together, we have

$$
n+K C_{K}+\delta=0
$$

therefore:

$$
\delta=-\left(n+K C_{K}\right)
$$

5. Using (4.3.8) in the second portion of equation (4.3.5), we then have:

$$
\sum_{i=1}^{n}\left(w_{i k}^{(m)}+\frac{C_{K}}{n}\right) \frac{1}{\pi_{k}}+\delta=\sum_{i=1}^{n}\left(w_{i k}^{(m)}+\frac{C_{K}}{n}\right) \frac{1}{\pi_{k}}-\left(n+K C_{K}\right)=0
$$

Rearranging (4.3.9), we obtain the updating equation (4.3.3)

Next, we need to update $\mu_{k}^{(m+1)}$ or $\beta_{k}^{(m+1)}, k=1, \ldots \ldots, K$ by maximizing the conditional expectation with respect to each scale parameter $\beta_{k}$ of the mixture 
component. The conditions imposed on the penalty function $p($.$) require that$ $p(0)=0$ and that it be a non-decreasing function on $(0,+\infty)$. Thus $p($.$) is not differentiable$ at $\eta_{k}=0$. The updated values can not be obtained using the derivatives. Fan and $\mathrm{Li}$ (2001) suggested using the following approximation of $p($.$) when the penalized values are$ bigger than the threshold.

$$
p^{*}\left(\eta_{k} ; \eta_{k}^{(m)}\right)=p\left(\eta_{k}^{(m)}\right)+\frac{p^{\prime}\left(\eta_{k}^{(m)}\right)}{2 \eta_{k}^{(m)}}\left(\eta^{2}-\eta_{k}^{(m)^{2}}\right)
$$

When the penalized values are less than the threshold, they suggest simply setting the value to zero which is equivalent to setting two adjacent means to the same value in our particular case. For the hard penalty term, when the penalized value is bigger than the threshold, above (4.3.10) becomes zero because the slope for a constant is zero.

Therefore, the update of $\mu_{k}^{(m+1)}$ or $\beta_{k}^{(m+1)}, k=1, \ldots \ldots, K$ can be obtained as in a normal EM algorithm. That is, find the update of $\beta_{k}^{(m+1)}, k=1, \ldots \ldots, K$ by maximizing following equation:

$$
\sum_{i=1}^{n} w_{i 1}^{(m)} \frac{\partial}{\partial \beta_{1}} \log \left\{f\left(y_{i} \mid \beta_{k}\right)\right\}=0
$$

The solution of the following equation produces the update values of $\beta_{k}^{(m+1)}, k=1, \ldots \ldots, K$ for the next iteration.

$$
\frac{\partial}{\partial \beta_{k}} \sum_{i=1}^{n} w_{i k}^{(m)} \log \left\{f\left(y_{i} \mid \beta_{k}\right)\right\}=-\frac{n \alpha}{\beta_{k}}+\frac{\sum_{i=1}^{n} y_{i}}{\beta_{k}^{2}}=0
$$

therefore: $\beta_{k}^{(m+1)}=\frac{\sum_{i=1}^{n} y_{i}}{n \alpha}$ or $\mu_{k}^{(m+1)}=\frac{\sum_{i=1}^{n} y_{i}}{n}$. 
The algorithm starts with an initial set of parameter values, then iterates between the $\mathrm{E}$ step and $\mathrm{M}$ step until the conditional expectation converges. After all iteration completed, the number of distinct values of the scale (or mean) parameters is obtained as the estimated order for a particular gene. This procedure runs on each gene one at a time. The threshold value of first penalty term is specified for each gene according to the shape parameter value and its sample size. The constant for the second penalty term is chosen for all genes of an experiment. The details of how to choose the threshold for the penalty terms is discussed in next section.

\subsection{Choices of the Threshold Parameters}

For the algorithm to run, the two threshold parameters need to be specified at the beginning. For $C_{K}$, both Chen et al. (2001) and Chen and Khalili (2005) reported that the choice of its value is not crucial, which is the case found in our computation. Here, we adopt the specification of MacKay (2002) to let $C_{K}=0.01 \frac{\log (n)}{\sqrt{n}}$. The sample size is the only information needed. Because normally all genes measured in a microarray experiment share the same sample size, the constant is specified once for all genes within an experiment.

As pointed out by Chen and Khalili (2005), the specification of the threshold for the first penalty term is theoretically difficult. Current development only provides some guidance on the choice of threshold to achieve consistency, which requires the threshold 
to shrink as the sample size increases. This guidance may be too general to help in real data analysis situations.

With the typical small sample size, the selection of the threshold becomes even more difficult. Based on this situation, we would like to propose a simulation-based approach specially designed for microarray data. We will discuss two possible ways to specify the penalty threshold for the first penalty term. The first one is developed specifically for microarray time course data analysis. The second one is for more general purposes and requires a larger sample size. As our focus is obviously the first one, we will only provide a brief discussion for the second one.

Microarray time course data has very limited sample size and even fewer time points. For these kinds of data, it is difficult to provide enough information for both order estimation and threshold specification. Although the number of genes is large, the separation threshold is gene specific and should not be determined by combining information from other genes. Besides the possible biological variation involved in an experiment, many other sources of technical variation may potentially affect the underlying separation of the expressions. On the other hand, it is generally understood that microarray analysis is designed to obtain a subset of genes for further investigation. Therefore, the primary goal of our analysis is to separate the set of genes with order one from those genes with order two or above. The set of genes with order two or above is the response set which is of major biological interest. For the response genes, whether their order is two or three or above is not critical, not only because the estimation has to be based on a very limited sample size and thus its accuracy is difficult to control, but also due to the fact the resulting response gene set needs to be verified by further detailed 
quantitative experimental measurements. Based on these considerations, we develop an approach suitable for microarray time course data analysis. We refer to it as the simulation based approach, which enables us to specify the threshold by simulation.

The focus for our method is to divide the genes into the set with order one from the set with multiple hidden states. The response genes are characterized by their change of state during a certain period of time. The identification of genes of order greater than one is sufficient for extracting the response gene set. For this purpose, as we have shown below, typical microarray time course data provide enough information. On the hand other, the goal of finding the exact number of hidden states may not be achievable using microarray data only. Even if we can obtain an estimate, how reliable it is by looking at only several biological replicates is still unknown.

The simulation based approach to specify a threshold as we introduce here also makes it possible to control for a desired type I error rate. When the shape parameter of a gene and the sample size are given, samples with a single underlying gamma distribution can be generated and our estimating method can be applied to find the order. In this way the falsely discovered multiple order samples can be identified and the accuracy of the order estimates can be obtained. We then can find a threshold value that has the corresponding type I error rate that we wish. The scale parameter value is not critical for maintaining a certain level of type I error rate based on our simulation. A few scale parameter values can be tried with a given shape parameter and sample size to evaluate the averaged number of falsely discovered samples. We will demonstrate this approach using the simulations in chapter 5 and using the real data in chapter 6 . 
In applications where the estimate of the exact number of hidden states is of interest, cross validation (Stone, 1974) and generalized cross validation (Craven and Wahba, 1979) are often used as data-driven approaches to choosing the threshold values. To use these cross-validation approaches, the data set needs to be randomly divided into two subsets, typically referred to as the training set and test set. There is no fully objective criterion developed for the threshold specification to date. These two data driven approaches still have some problems. The threshold found in this way is a random variable which may not satisfy the asymptotic properties required for the penalty terms. To ensure the validity of the asymptotic results, normally a restriction is imposed on the values found by cross-validation approaches (James et al., 2001). In addition, these approaches normally require intensive computation and make it too difficult to be applied on the large number of genes from microarray data.

All above computations are implemented using $\mathbf{R}$ language version 2.3.1 and later versions. The $\mathrm{R}$ code is attached in Appendix B.

\subsection{Identifiability}

To estimate the parameters for the model (4.2.2), we need to first make sure that the model parameters $\Psi$ are identifiable. According to McLachlan and Peel (2000), in general, a parametric family of densities $f\left(y_{i} \mid \Psi\right)$ is identifiable if distinct values of the parameters $\Psi$ determine distinct family of models 
$\left\{f\left(y_{i} \mid \Psi\right): \Psi \in \Omega\right\}$

where $\Omega$ is the parameter space. That is

$$
\begin{aligned}
& \text { if } \Psi \neq \Psi^{*}, \\
& \text { then } f\left(y_{i} \mid \Psi\right) \neq f\left(y_{i} \mid \Psi^{*}\right)
\end{aligned}
$$

For parametric distributions such as the normal distribution and gamma distribution, the parameters are identifiable in the single distribution situation. However, the identifiability becomes an issue in the mixture model situation when we do not know which component distribution generates an observation. The identifiability problem is discussed here mostly in a mixture model situation.

In mixture distribution situation, the identified parameters for the component distributions can be re-labeled using any permutation of the labels. The same group of parameters can be regarded as different when labeled differently. To avoid this problem, in the mixture distribution situation, one normally imposes an ordering restriction, as demonstrated by Aitkin and Rubin (1985), to order the parameters. After ordering, the parameters can be compared to see whether the condition of identifiability is satisfied. Two groups of parameters with the same values after ordering are always the same. The identifiability problem discussed below is for the situation where the lack of identifiability can not be resolved only by ordering the parameters.

Typically, lack of identifiability occurs in estimating parameters for mixture distributions, due to over fitting. That is, a larger than true order is used in estimating the parameters. In such a situation, one of following is expected to happen: 
1. One of the mixing proportions is actually estimating a zero proportion.

2. Two different component distributions are actually estimating the same distribution.

These two situations cause an identifiability problem in estimation of the mixture models. The parameters for a component whose proportion is zero can be estimated in many different ways. Similarly, for two estimated components which are actually estimating the same distribution, their mixing proportions can be estimated in many ways. The two penalty terms used in (4.2.2) are developed to prevent these two possible situations of over fitting. The first penalty term in (4.2.2) is designed to prevent two mixture components from being estimated for the same distribution. The second penalty term is designed to prevent any mixing proportion from being estimated to be too close to zero.

There is an important condition for the lack of identifiability. That is, lack of identifiability occurs only when we have limited information from the sampled data. Asymptotically, the above two possible estimation problems can be avoided. Lack of identifiability is a problem introduced by the limited information from a finite sample. Under such information limitation, there may be at least two different parameter sets providing the same likelihood measurement for a given set of observed data. 


\section{Chapter 5}

\section{SIMULATION STUDY}

The objective of the simulation study in this chapter is to evaluate the performance of our proposed method with a focus on our primary goal. The primary objective of our analysis and simulation is to demonstrate how the threshold for the first penalty term can be specified using simulated data. The simulations also provide evaluation of the performance of our method for microarray data. The secondary objective is to provide some evaluations for the situation where we know the separation threshold among mixture components, which is not the case for microarray experiments. Therefore these simulations are designed to reflect general goals instead of specifically for microarray data. Accordingly, the data are simulated directly from the conditions we used in the development of our model rather than for the microarray situation. We also include simulations based on hidden Markov models. 
We first use our simulation based approach to find a value for the separation threshold. This is consistent with our primary objective in this thesis. Our proposed order estimation combined with the simulation based approach for threshold specification makes it especially suitable for analyzing microarray time course data.

Because microarray experiments are used as screening tools, it is usually the case that we do not have any information about the separation threshold. Even if we do know the threshold information for some genes, with such small sample size, the order estimation can not be expected to be very reliable. This is the case as we will show using simulation with small samples. Therefore, the characterization of the exact order for a gene has to be conducted in two steps. First, narrow down the collection of genes to a subset of genes which potentially have two or more states. Second, perform more detailed measurements on those genes selected in the first stage, with sufficiently large sample size, using some other experiments. Here our focus is on the first step. We only include a brief discussion of estimating the exact order for situations with sufficiently large sample size in the simulation. Furthermore, in our opinion, to estimate the exact order using microarray data is not achievable and thus is not an objective of our method.

The detailed situation is illustrated in Table 5.1. The classification of genes into those with order one and those with order two or above can be achieved for both small samples and medium to large samples. The estimation of the exact order only performs well with medium to large samples. Hence, our simulation will be performed mainly for the estimation aimed to identify response genes with order of two or above. We also 
provide some simulations and a brief discussion for the situations where one is estimating the exact order for medium to large sample sizes.

Table 5.1, The four situations of order estimation.

\begin{tabular}{|c|c|c|}
\hline & $\begin{array}{c}\text { Separate order of one from } \\
\text { the order of two or above }\end{array}$ & Find the exact order \\
\hline Small sample size & Achievable & Not Achievable \\
\hline Medium to large sample & Achievable & Achievable \\
\hline
\end{tabular}

A common approach to deal with the typical small sample sizes in microarray literature is to use the Bayesian method to borrow information from other genes. This can not be used here because as we explained in the first chapter, the gene context effect is always gene specific and need to be investigated first. Even if a group of genes shares the same order, this does not guarantee their data are combinable. This is because the shared number of hidden states can result from completely different shape parameter and mean parameter values. Their underlying separation probability can be also very different. Supporting evidence found from biological experiments indicates that different genes have different basal expression levels, indicating that their smallest mean parameters are potentially different. In real situations, without knowing whether the shape parameter, mean parameters and the separation probability are similar or not, combining information from different genes is difficult to justify. 
For a given sample size and shape parameter, a separation threshold can be chosen to maintain a type I error rate level. The procedure is:

1. Find the sample size and shape parameter for a specific gene from the analysis of microarray time course data found in previous chapters.

2. Choose several mean parameter values within the expression range of the gene. The scale parameters can be chosen accordingly with a known shape parameter. These values, as we shall discover from our simulations, are not critical.

3. Simulate a large number of samples (we use 500 samples per simulation) of a single gamma distribution with the mean (or scale parameter), shape parameter and sample size as specified as in step 2 .

4. Run our order estimation algorithm with a threshold value and find how many samples are found to have order estimated as two or above. The proportion of falsely discovered high order samples (order equals two or above) is recorded for each simulation.

5. For a desired type I error rate value, find the corresponding separation threshold which leads to the desired type I error rate value. As an example, the threshold probability for a $3 \%$ type I error rate is in the range from $68 \%$ to $84 \%$ as shown in Table 5.2 .

The proportion of falsely discovered samples (i.e. the number of samples found to have more than one hidden state) is compared to a targeted level. We expect on average that the proportion of falsely discovered response genes is under a specified level if the same situation is repeated many times. 
As shown in Table 5.2, the type I error rate can be effectively controlled for a specific shape parameter and a range of mean parameter values. We generate 500 samples from a single gamma distribution with its shape and scale parameters as specified in each row of Table 5.2. The mean parameter (not shown) is the product of the shape and scale parameters. The sample size of each of the 500 samples is chosen as 20 to represent a typical microarray time course situation. At each specification of the shape and scale parameter, three separation threshold values are chosen as examples. Our algorithm then computes the order and finds the proportion of samples with more than one hidden state, as listed in Table 5.2. Since all of the samples are generated from a single underlying gamma distribution, the number recorded in the table divided by 500 provides the proportion of the falsely discovered samples whose order is higher than 1 . Using 3\% type I error rate as an example, we mark the corresponding separation threshold values with a star which have the type I error rate controlled at about $3 \%$ level.

For different genes, because the estimate of shape parameter is different, the simulation needs to be performed again in the same way. The type I error rate value can also be chosen at a different type I error rate level for different genes. Here we use the same type I error rate level across all genes for ease of illustration. The threshold values with a star in Table 5.2 are the separation threshold values that we used in the real data analysis in chapter 6 . 
Table 5.2. Type I error rate at three separation thresholds for various single gamma distributions.

\begin{tabular}{|c|c|c|c|}
\hline True Order & gamma (shape $\alpha$, scale $\beta$ parameter) & Separation Threshold & $\begin{array}{l}\text { False Discovered } \\
\text { proportion among } 500 \\
\text { samples }\end{array}$ \\
\hline 1 & $(60,10)$ & $73 \%$ & 0.016 \\
\hline 1 & $(60,10)$ & $* 72 \%$ & 0.026 \\
\hline 1 & $(60,10)$ & $71 \%$ & 0.044 \\
\hline 1 & $(60,15)$ & $73 \%$ & 0.008 \\
\hline 1 & $(60,15)$ & $* 72 \%$ & 0.02 \\
\hline 1 & $(60,15)$ & $71 \%$ & 0.028 \\
\hline 1 & $(60,30)$ & $73 \%$ & 0.012 \\
\hline 1 & $(60,30)$ & $* 72 \%$ & 0.016 \\
\hline 1 & $(60,30)$ & $71 \%$ & 0.03 \\
\hline 1 & $(40,10)$ & $73 \%$ & 0.004 \\
\hline 1 & $(40,10)$ & $* 72 \%$ & 0.028 \\
\hline 1 & $(40,10)$ & $71 \%$ & 0.05 \\
\hline 1 & $(40,15)$ & $73 \%$ & 0.012 \\
\hline 1 & $(40,15)$ & $* 72 \%$ & 0.026 \\
\hline 1 & $(40,15)$ & $71 \%$ & 0.048 \\
\hline 1 & $(40,30)$ & $73 \%$ & 0.01 \\
\hline 1 & $(40,30)$ & $* 72 \%$ & 0.018 \\
\hline 1 & $(40,30)$ & $71 \%$ & 0.03 \\
\hline 1 & $(20,10)$ & $74 \%$ & 0.01 \\
\hline 1 & $(20,10)$ & $* 73 \%$ & 0.022 \\
\hline
\end{tabular}




\begin{tabular}{|c|c|c|c|}
\hline 1 & $(20,10)$ & $72 \%$ & 0.04 \\
\hline 1 & $(20,15)$ & $74 \%$ & 0.01 \\
\hline 1 & $(20,15)$ & $* 73 \%$ & 0.022 \\
\hline 1 & $(20,15)$ & $72 \%$ & 0.04 \\
\hline 1 & $(20,30)$ & $74 \%$ & 0.016 \\
\hline 1 & $(20,30)$ & $* 73 \%$ & 0.034 \\
\hline 1 & $(20,30)$ & $72 \%$ & 0.048 \\
\hline 1 & $(15,10)$ & $75 \%$ & 0.006 \\
\hline 1 & $(15,10)$ & $* \overline{74 \%}$ & 0.02 \\
\hline 1 & $(15,10)$ & $73 \%$ & 0.038 \\
\hline 1 & $(15,15)$ & $75 \%$ & 0.002 \\
\hline 1 & $(15,15)$ & $* 74 \%$ & 0.01 \\
\hline 1 & $(15,15)$ & $73 \%$ & 0.018 \\
\hline 1 & $(15,30)$ & $75 \%$ & 0.004 \\
\hline 1 & $(15,30)$ & *74\% & 0.016 \\
\hline 1 & $(15,30)$ & $73 \%$ & 0.028 \\
\hline 1 & $(10,10)$ & $76 \%$ & 0.01 \\
\hline 1 & $(10,10)$ & *75\% & 0.028 \\
\hline 1 & $(10,10)$ & $74 \%$ & 0.038 \\
\hline 1 & $(10,15)$ & $76 \%$ & 0.01 \\
\hline 1 & $(10,15)$ & ${ }^{*} 75 \%$ & 0.02 \\
\hline 1 & $(10,15)$ & $74 \%$ & 0.036 \\
\hline 1 & $(10,30)$ & $76 \%$ & 0.012 \\
\hline 1 & $(10,30)$ & ${ }^{*} 75 \%$ & 0.018 \\
\hline 1 & $(10,30)$ & $74 \%$ & 0.024 \\
\hline
\end{tabular}




\begin{tabular}{|c|c|c|c|}
\hline 1 & $(8,10)$ & $76 \%$ & 0.01 \\
\hline 1 & $(8,10)$ & $* 75 \%$ & 0.024 \\
\hline 1 & $(8,10)$ & $74 \%$ & 0.048 \\
\hline 1 & $(8,15)$ & $76 \%$ & 0.01 \\
\hline 1 & $(8,15)$ & ${ }^{*} 75 \%$ & 0.03 \\
\hline 1 & $(8,15)$ & $74 \%$ & 0.048 \\
\hline 1 & $(8,30)$ & $76 \%$ & 0.016 \\
\hline 1 & $(8,30)$ & $* 75 \%$ & 0.032 \\
\hline 1 & $(8,30)$ & $74 \%$ & 0.06 \\
\hline 1 & $(5,10)$ & $78 \%$ & 0.01 \\
\hline 1 & $(5,10)$ & $* 77 \%$ & 0.026 \\
\hline 1 & $(5,10)$ & $76 \%$ & 0.036 \\
\hline 1 & $(5,15)$ & $78 \%$ & 0.01 \\
\hline 1 & $(5,15)$ & $* 77 \%$ & 0.016 \\
\hline 1 & $(5,15)$ & $76 \%$ & 0.032 \\
\hline 1 & $(5,30)$ & $78 \%$ & 0.012 \\
\hline 1 & $(5,30)$ & $* 77 \%$ & 0.022 \\
\hline 1 & $(5,30)$ & $76 \%$ & 0.046 \\
\hline 1 & $(3,10)$ & $80 \%$ & 0.014 \\
\hline 1 & $(3,10)$ & $* 79 \%$ & 0.028 \\
\hline 1 & $(3,10)$ & $78 \%$ & 0.038 \\
\hline 1 & $(3,15)$ & $80 \%$ & 0.022 \\
\hline 1 & $(3,15)$ & $* 79 \%$ & 0.028 \\
\hline 1 & $(3,15)$ & $78 \%$ & 0.048 \\
\hline 1 & $(3,30)$ & $80 \%$ & 0.014 \\
\hline
\end{tabular}




\begin{tabular}{|l|l|l|l|}
\hline 1 & $(3,30)$ & $* 79 \%$ & 0.02 \\
\hline 1 & $(3,30)$ & $78 \%$ & 0.058 \\
\hline 1 & $(2,10)$ & $82 \%$ & 0.016 \\
\hline 1 & $(2,10)$ & $* 81 \%$ & 0.022 \\
\hline 1 & $(2,10)$ & $80 \%$ & 0.046 \\
\hline 1 & $(2,15)$ & $82 \%$ & 0.02 \\
\hline 1 & $(2,15)$ & $* 81 \%$ & 0.04 \\
\hline 1 & $(2,15)$ & $80 \%$ & 0.064 \\
\hline 1 & $(2,30)$ & $82 \%$ & 0.016 \\
\hline 1 & $(2,30)$ & $* 81 \%$ & 0.02 \\
\hline 1 & $(2,30)$ & $80 \%$ & 0.038 \\
\hline 1 & $(1,10)$ & $87 \%$ & 0.014 \\
\hline 1 & $(1,10)$ & $* 86 \%$ & 0.024 \\
\hline 1 & $(1,10)$ & $85 \%$ & 0.036 \\
\hline 1 & $(1,15)$ & $87 \%$ & 0.012 \\
\hline 1 & $(1,15)$ & $* 86 \%$ & 0.02 \\
\hline 1 & $(1,15)$ & $85 \%$ & 0.042 \\
\hline 1 & $(1,30)$ & $87 \%$ & 0.026 \\
\hline 1 & $(1,30)$ & $886 \%$ & 0.032 \\
\hline 1 & $(1,30)$ & 0.052 \\
\hline
\end{tabular}

The type I error rate of our method is an important tool to control the performance of the procedure for distinguishing genes with only one state from those genes with multiple states. Further characterization of the number of states for the multiple state 
genes, can be investigated in more detail by employing other biological techniques. The above simulations demonstrate that the separation threshold can be determined to effectively control the type I error rate for a specific sample size. The type I error rate in the above table is also an indicator of the accuracy that our proposed method can achieve.

As shown in Table 5.2, for a given shape parameter, a corresponding separation threshold level can be found to keep the type I error rate at a desired level for various values of the scale parameter or mean parameter. This confirms that for a given shape parameter, the separation threshold can be used to consistently control the type I error rate for various values of the scale parameter or mean parameter. On the other hand, as the shape parameter varies, the separation probability needs to be adjusted to maintain a specified type I error rate. A larger separation threshold value is needed for a smaller shape parameter. In Table 5.2, a series of separation probability thresholds are obtained for different shape parameter values for the analysis of real microarray time course data in chapter 6 . We expect to keep the type I error rate near or under $3 \%$ for all genes with different shape parameters in the real data analysis in the next chapter.

Here we also provide some evaluations for the situation where the exact number of states is of interest. In such cases, our order estimation procedure can be used to find out the order of a hidden Markov model or a finite mixture model. As we discussed above, the separation probability for microarray data is unknown. The mixing proportion is also unknown. Simulations are unable to be performed for real microarray data. Instead, simulations are performed for general situations where sufficiently large samples are 
available and separation probability is known. The data are also assumed to come from stationary HMMs or a finite mixture models and homogeneous HMMs.

We use a small sample size of 20 and a medium sample size of 300 to show that exact order estimation requires a sufficiently large sample size, given a known separation threshold. The difficulty of exact order estimation comes from not only the lack of sophisticated theoretical tools but also the lack of information contained in a given sample. We briefly present simulations in Table 5.4 where the true order is 2 and in Table 5.5 where the true order is 3 with both sample size of 20 and 300 . Each run in Table 5.4 and Table 5.5 consists of 500 samples. We selected the separation probability in the range from $72 \%$ to $99 \%$, as examples.

Table 5.3 explains how the data are generated for Table 5.4 and Table 5.5. The first three columns specify the sample size, the true order and the separation probability for the mixtures. The distance between two adjacent gamma distributions is defined by the separation probability in the third column. The mixing proportion is specified in the fourth column. The parameters of the gamma mixture components are listed in the fifth and sixth columns. 
Table 5.3. The specifications for the simulation of mixtures of two gamma distributions. The sample size, true order, separation threshold, mixing proportion and the parameters of each gamma distribution with 500 samples are listed in each row. The estimated order is given in Table 5.4 and Table 5.5.

\begin{tabular}{|l|l|l|l|l|l|}
\hline Sample size & $\begin{array}{l}\text { True } \\
\text { order }\end{array}$ & $\begin{array}{l}\text { Separation } \\
\text { prob. }\end{array}$ & $\begin{array}{l}\text { Mixing } \\
\text { proportion }\end{array}$ & $\begin{array}{l}\text { First gamma } \\
\text { Dist. (shape } \\
\alpha, \text { scale } \beta)\end{array}$ & $\begin{array}{l}\text { Second } \\
\text { gamma Dist. } \\
\text { shape } \alpha, \\
\text { scale } \beta)\end{array}$ \\
\hline 20 & 2 & $72 \%$ & $0.4,0.6$ & $(40,15)$ & $(40,17.85)$ \\
\hline 20 & 2 & $82 \%$ & $0.4,0.6$ & $(40,15)$ & $(40,19.8)$ \\
\hline 20 & 2 & $92 \%$ & $0.4,0.6$ & $(40,15)$ & $(40,23.1)$ \\
\hline 20 & 2 & $73 \%$ & $0.4,0.6$ & $(20,15)$ & $(20,19.2)$ \\
\hline 20 & 2 & $83 \%$ & $0.4,0.6$ & $(20,15)$ & $(20,22.35)$ \\
\hline 20 & 2 & $93 \%$ & $0.4,0.6$ & $(20,15)$ & $(20,28.2)$ \\
\hline 20 & 2 & $76 \%$ & $0.4,0.6$ & $(10,15)$ & $(10,22.05)$ \\
\hline 20 & 2 & $86 \%$ & $0.4,0.6$ & $(10,15)$ & $(10,28.05)$ \\
\hline 20 & 2 & $96 \%$ & $0.4,0.6$ & $(10,15)$ & $(10,43.05)$ \\
\hline 20 & 2 & $79 \%$ & $0.4,0.6$ & $(5,15)$ & $(5,27.15)$ \\
\hline 20 & 2 & $89 \%$ & $0.4,0.6$ & $(5,15)$ & $(5,39.75)$ \\
\hline 20 & 2 & $99 \%$ & $0.4,0.6$ & $(5,15)$ & $(5,112.05)$ \\
\hline 300 & 2 & $72 \%$ & $0.4,0.6$ & $(40,15)$ & $(40,17.85)$ \\
\hline 300 & 2 & $82 \%$ & $0.4,0.6$ & $(40,15)$ & $(40,19.8)$ \\
\hline 300 & 2 & $92 \%$ & $0.4,0.6$ & $(40,15)$ & $(40,23.1)$ \\
\hline 300 & 2 & $73 \%$ & $0.4,0.6$ & $(20,15)$ & $(20,19.2)$ \\
\hline 300 & 2 & $83 \%$ & $0.4,0.6$ & $(20,15)$ & $(20,22.35)$ \\
\hline 300 & 2 & $93 \%$ & $0.4,0.6$ & $(20,15)$ & $(20,28.2)$ \\
\hline 300 & 2 & $76 \%$ & $0.4,0.6$ & $(10,15)$ & $(10,22.05)$ \\
\hline 300 & 2 & $86 \%$ & $0.4,0.6$ & $(10,15)$ & $(10,28.05)$ \\
\hline 300 & 2 & $96 \%$ & $0.4,0.6$ & $(10,15)$ & $(10,43.05)$ \\
\hline 300 & 2 & $79 \%$ & $0.4,0.6$ & $(5,15)$ & $(5,27.15)$ \\
\hline 300 & 2 & $89 \%$ & $0.4,0.6$ & $(5,15)$ & $(5,39.75)$ \\
\hline 300 & 2 & $99 \%$ & $0.4,0.6$ & $(5,15)$ & $(5,112.05)$ \\
\hline & & & & \\
\hline
\end{tabular}

The data in Table 5.4 and Table 5.5 are generated according to the listed specifications and the following steps:

1. Use the multinomial distribution with the specified mixing proportions to generate the number of observations for each gamma component. 
2. Generate the observations for each component distribution with the specified shape and scale parameters and with the number of observations decided by step 1 .

3. Mix the data points together.

This data generation procedure is used to generate gamma mixtures with order 2 and 3 for the evaluations in Table 5.4 and Table 5.5.

In Table 5.4 and Table 5.5, the data are generated from mixture models (or equivalently from a HMM with a stationary probability) specified as in each row of the table. The estimated order is reported as a proportion of 500 samples. Assume that the underlying separation probability is known. A separation threshold value is chosen as an example. For illustration purposes, we select a value slightly smaller than the true underlying separation probability to account for sample variation. The probability threshold used in Table 5.4 and Table 5.5 is among many possible values that can be specified. When the threshold is specified bigger than the true threshold, the correct estimation proportion could be very low and close to zero (data not shown). The threshold value is picked as an example. There are many possible threshold values which could be chosen in Table 5.4 and Table 5.5. The values used serve only as an example. 
Table 5.4. Simulation evaluations for the mixture models with 2 components.

\begin{tabular}{|c|c|c|c|c|c|c|c|c|c|}
\hline $\begin{array}{l}\text { Sample } \\
\text { size }\end{array}$ & $\begin{array}{l}\text { True } \\
\text { order }\end{array}$ & $\begin{array}{c}\text { Sep. } \\
\text { Threshold }\end{array}$ & $\begin{array}{l}\text { Sep. } \\
\text { prob. }\end{array}$ & $\begin{array}{l}\text { Mixing } \\
\text { proportion }\end{array}$ & $\begin{array}{c}\text { First } \\
\text { gamma } \\
\text { Dist. } \\
\text { (shape } \alpha \text {, } \\
\text { scale } \beta \text { ) }\end{array}$ & $\begin{array}{c}\text { Second } \\
\text { gamma Dist. } \\
\text { (shape } \alpha \\
\text { scale } \beta \text { ) }\end{array}$ & $\begin{array}{c}\begin{array}{c}\text { Among } \\
500 \\
\text { repeats, } \\
\text { the } \\
\text { proportion } \\
\text { of order }<2\end{array} \\
\end{array}$ & $\begin{array}{l}\text { Among } 500 \\
\text { repeats, } \\
\text { the } \\
\text { proportion } \\
\text { of order }=2\end{array}$ & $\begin{array}{c}\text { Among } 500 \\
\text { repeats, } \\
\text { the } \\
\text { proportion } \\
\text { of order }>2\end{array}$ \\
\hline 20 & 2 & $60 \%$ & $72 \%$ & $0.4,0.6$ & $(40,15)$ & $(40,17.85)$ & 0.276 & 0.578 & 0.146 \\
\hline 20 & 2 & $62 \%$ & $82 \%$ & $0.4,0.6$ & $(40,15)$ & $(40,19.8)$ & 0.074 & 0.682 & 0.244 \\
\hline 20 & 2 & $68 \%$ & $92 \%$ & $0.4,0.6$ & $(40,15)$ & $(40,23.1)$ & 0.032 & 0.60 & 0.368 \\
\hline 20 & 2 & $63 \%$ & $73 \%$ & $0.4,0.6$ & $(20,15)$ & $(20,19.2)$ & 0.304 & 0.574 & 0.122 \\
\hline 20 & 2 & $63 \%$ & $83 \%$ & $0.4,0.6$ & $(20,15)$ & $\begin{array}{c}(20, \\
22.35)\end{array}$ & 0.088 & 0.666 & 0.246 \\
\hline 20 & 2 & $63 \%$ & $93 \%$ & $0.4,0.6$ & $(20,15)$ & $(20,28.2)$ & 0.004 & 0.53 & 0.466 \\
\hline 20 & 2 & $66 \%$ & $76 \%$ & $0.4,0.6$ & $(10,15)$ & $(10,22.05)$ & 0.332 & 0.558 & 0.11 \\
\hline 20 & 2 & $66 \%$ & $86 \%$ & $0.4,0.6$ & $(10,15)$ & $(10,28.05)$ & 0.072 & 0.64 & 0.288 \\
\hline 20 & 2 & $66 \%$ & $96 \%$ & $0.4,0.6$ & $(10,15)$ & $(10,43.05)$ & 0 & 0.552 & 0.448 \\
\hline 20 & 2 & $69 \%$ & $79 \%$ & $0.4,0.6$ & $(5,15)$ & $(5,27.15)$ & 0.3 & 0.568 & 0.132 \\
\hline 20 & 2 & $69 \%$ & $89 \%$ & $0.4,0.6$ & $(5,15)$ & $(5,39.75)$ & 0.038 & 0.676 & 0.286 \\
\hline 20 & 2 & $69 \%$ & $99 \%$ & $0.4,0.6$ & $(5,15)$ & $(5,112.05)$ & 0 & 0.534 & 0.466 \\
\hline 300 & 2 & $66 \%$ & $72 \%$ & $0.4,0.6$ & $(40,15)$ & $(40,17.85)$ & 0.15 & 0.79 & 0.06 \\
\hline 300 & 2 & $70 \%$ & $82 \%$ & $0.4,0.6$ & $(40,15)$ & $(40,19.8)$ & 0.082 & 0.74 & 0.178 \\
\hline 300 & 2 & $76 \%$ & $92 \%$ & $0.4,0.6$ & $(40,15)$ & $(40,23.1)$ & 0.086 & 0.822 & 0.092 \\
\hline 300 & 2 & $66 \%$ & $73 \%$ & $0.4,0.6$ & $(20,15)$ & $(20,19.2)$ & 0.114 & 0.722 & 0.164 \\
\hline 300 & 2 & $71 \%$ & $83 \%$ & $0.4,0.6$ & $(20,15)$ & $\begin{array}{c}(20, \\
22.35)\end{array}$ & 0.106 & 0.712 & 0.182 \\
\hline 300 & 2 & $76 \%$ & $93 \%$ & $0.4,0.6$ & $(20,15)$ & $(20,28.2)$ & 0.016 & 0.796 & 0.188 \\
\hline 300 & 2 & $70 \%$ & $76 \%$ & $0.4,0.6$ & $(10,15)$ & $(10,22.05)$ & 0.108 & 0.726 & 0.166 \\
\hline 300 & 2 & $74 \%$ & $86 \%$ & $0.4,0.6$ & $(10,15)$ & $(10,28.05)$ & 0.168 & 0.636 & 0.196 \\
\hline 300 & 2 & $79 \%$ & $96 \%$ & $0.4,0.6$ & $(10,15)$ & $(10,43.05)$ & 0 & 0.814 & 0.186 \\
\hline 300 & 2 & $74 \%$ & $79 \%$ & $0.4,0.6$ & $(5,15)$ & $(5,27.15)$ & 0.098 & 0.664 & 0.238 \\
\hline
\end{tabular}




\begin{tabular}{|c|c|c|c|c|c|c|c|c|c|}
\hline 300 & 2 & $77 \%$ & $89 \%$ & $0.4,0.6$ & $(5,15)$ & $(5,39.75)$ & 0.198 & 0.562 & 0.24 \\
\hline 300 & 2 & $82 \%$ & $99 \%$ & $0.4,0.6$ & $(5,15)$ & $(5,112.05)$ & 0 & 0.82 & 0.18 \\
\hline
\end{tabular}

Similar to above simulation, data was also generated from a mixture when the true order is 3. The result of the estimation is shown in Table 5.5.

Table 5.5. Simulation evaluations for the mixture models with 3 components.

\begin{tabular}{|c|c|c|c|c|c|c|c|c|c|c|}
\hline 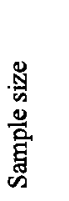 & 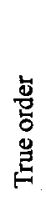 & 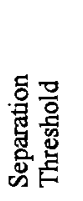 & 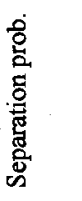 & 总总 & 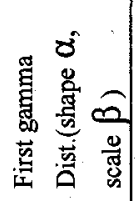 & 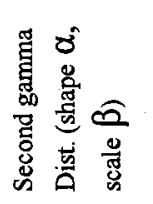 & 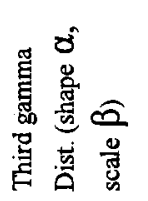 & 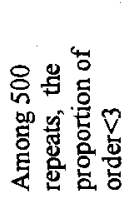 & 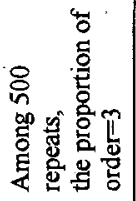 & 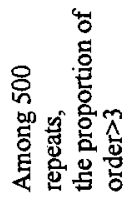 \\
\hline 20 & 3 & $56 \%$ & $72 \%$ & $\begin{array}{c}0.2,0.3 \\
0.5\end{array}$ & $(40,15)$ & $(40,17.85)$ & $\begin{array}{c}(40 \\
21.24)\end{array}$ & 0.55 & 0.33 & 0.12 \\
\hline 20 & 3 & $60 \%$ & $82 \%$ & $\begin{array}{c}0.2,0.3 \\
0.5\end{array}$ & $(40,15)$ & $(40,19.8)$ & $\begin{array}{c}(40, \\
26.14)\end{array}$ & 0.376 & 0.48 & 0.144 \\
\hline 20 & 3 & $68 \%$ & $92 \%$ & $\begin{array}{c}0.2,0.3 \\
0.5\end{array}$ & $(40,15)$ & $(40,23.1)$ & $\begin{array}{c}(40, \\
35.57)\end{array}$ & 0.276 & 0.632 & 0.092 \\
\hline 20 & 3 & $58 \%$ & $73 \%$ & $\begin{array}{c}0.2,0.3 \\
0.5\end{array}$ & $(20,15)$ & $(20,19.2)$ & $\begin{array}{c}(20 \\
24.58)\end{array}$ & 0.57 & 0.356 & 0.074 \\
\hline 20 & 3 & $61 \%$ & $83 \%$ & $\begin{array}{c}0.2,0.3 \\
0.5\end{array}$ & $(20,15)$ & $\begin{array}{c}(20, \\
22.35)\end{array}$ & $(20,33.3)$ & 0.37 & 0.506 & 0.124 \\
\hline 20 & 3 & $66 \%$ & $93 \%$ & $\begin{array}{c}0.2,0.3 \\
0.5\end{array}$ & $(20,15)$ & $(20,28.2)$ & $\begin{array}{c}(20, \\
53.02)\end{array}$ & 0.06 & 0.65 & 0.29 \\
\hline 20 & 3 & $58 \%$ & $76 \%$ & $\begin{array}{c}0.2,0.3 \\
0.5\end{array}$ & $(10,15)$ & $(10,22.05)$ & $\begin{array}{c}(10, \\
32.41)\end{array}$ & 0.538 & 0.33 & 0.132 \\
\hline 20 & 3 & $60 \%$ & $86 \%$ & $\begin{array}{c}0.2,0.3 \\
0.5\end{array}$ & $(10,15)$ & $(10,28.05)$ & $\begin{array}{c}(10, \\
52.45)\end{array}$ & 0.32 & 0.462 & 0.218 \\
\hline 20 & 3 & 0.66 & $96 \%$ & $\begin{array}{c}0.2,0.3 \\
0.5\end{array}$ & $(10,15)$ & $(10,43.05)$ & $\begin{array}{c}(10, \\
123.55)\end{array}$ & 0.022 & 0.52 & 0.458 \\
\hline 20 & 3 & $58 \%$ & $79 \%$ & $\begin{array}{c}0.2,0.3 \\
0.5\end{array}$ & $(5,15)$ & $(5,27.15)$ & $(5,49.14)$ & 0.45 & 0.328 & 0.222 \\
\hline 20 & 3 & $60 \%$ & $89 \%$ & $\begin{array}{c}0.2,0.3 \\
0.5\end{array}$ & $(5,15)$ & $(5,39.75)$ & $\begin{array}{c}(5 \\
105.34)\end{array}$ & 0.148 & 0.51 & 0.342 \\
\hline 20 & 3 & $68 \%$ & $99 \%$ & $\begin{array}{c}0.2,0.3 \\
0.5\end{array}$ & $(5,15)$ & $(5,112.05)$ & $\begin{array}{c}(5, \\
837.01)\end{array}$ & 0.006 & 0.402 & 0.592 \\
\hline 300 & 3 & $66 \%$ & $72 \%$ & $\begin{array}{c}0.2,0.3 \\
0.5\end{array}$ & $(40,15)$ & $(40,17.85)$ & $\begin{array}{c}(40, \\
21.24)\end{array}$ & 0.414 & 0.496 & 0.09 \\
\hline 300 & 3 & $70 \%$ & $82 \%$ & $\begin{array}{c}0.2,0.3 \\
0.5\end{array}$ & $(40,15)$ & $(40,19.8)$ & $\begin{array}{c}(40, \\
26.14)\end{array}$ & 0.058 & 0.62 & 0.322 \\
\hline 300 & 3 & $76 \%$ & $92 \%$ & $\begin{array}{c}0.2,0.3 \\
0.5\end{array}$ & $(40,15)$ & $(40,23.1)$ & $\begin{array}{c}(40, \\
35.57)\end{array}$ & 0 & 0.744 & 0.256 \\
\hline
\end{tabular}




\begin{tabular}{|c|c|c|c|c|c|c|c|c|c|c|}
\hline 300 & 3 & $66 \%$ & $73 \%$ & $\begin{array}{c}0.2,0.3, \\
0.5\end{array}$ & $(20,15)$ & $(20,19.2)$ & $\begin{array}{c}(20, \\
24.58)\end{array}$ & 0.27 & 0.532 & 0.20 \\
\hline 300 & 3 & $71 \%$ & $83 \%$ & $\begin{array}{c}0.2,0.3, \\
0.5\end{array}$ & $(20,15)$ & $\begin{array}{c}(20, \\
22.35)\end{array}$ & $(20,33.3)$ & 0.14 & 0.712 & 0.148 \\
\hline 300 & 3 & $80 \%$ & $93 \%$ & $\begin{array}{c}0.2,0.3, \\
0.5\end{array}$ & $(20,15)$ & $(20,28.2)$ & $\begin{array}{c}(20, \\
53.02)\end{array}$ & 0 & 0.892 & 0.108 \\
\hline 300 & 3 & $68 \%$ & $76 \%$ & $\begin{array}{c}0.2,0.3, \\
0.5\end{array}$ & $(10,15)$ & $(10,22.05)$ & $\begin{array}{c}(10, \\
32.41)\end{array}$ & 0.228 & 0.616 & 0.156 \\
\hline 300 & 3 & $76 \%$ & $86 \%$ & $\begin{array}{c}0.2,0.3, \\
0.5\end{array}$ & $(10,15)$ & $(10,28.05)$ & $\begin{array}{c}(10, \\
52.45)\end{array}$ & 0.03 & 0.80 & 0.17 \\
\hline 300 & 3 & $82 \%$ & $96 \%$ & $\begin{array}{c}0.2,0.3, \\
0.5\end{array}$ & $(10,15)$ & $(10,43.05)$ & $\begin{array}{c}(10, \\
123.55)\end{array}$ & 0 & 0.932 & 0.068 \\
\hline 300 & 3 & $72 \%$ & $79 \%$ & $\begin{array}{c}0.2,0.3, \\
0.5\end{array}$ & $(5,15)$ & $(5,27.15)$ & $(5,49.14)$ & 0.214 & 0.666 & 0.12 \\
\hline 300 & 3 & $80 \%$ & $89 \%$ & $\begin{array}{c}0.2,0.3, \\
0.5\end{array}$ & $(5,15)$ & $(5,39.75)$ & $\begin{array}{c}(5, \\
105.34)\end{array}$ & 0.02 & 0.84 & 0.14 \\
\hline 300 & 3 & $82 \%$ & $99 \%$ & $\begin{array}{c}0.2,0.3, \\
0.5\end{array}$ & $(5,15)$ & $(5,112.05)$ & $\begin{array}{c}(5, \\
837.01)\end{array}$ & 0 & 0.858 & 0.142 \\
\hline
\end{tabular}

In both Table 5.4 and Table 5.5, the percentage of correct estimates ranges from $32 \%$ to $93 \%$ and the proportion of correct estimates improves as the sample size increases. For sample size of 20 , the order is poorly estimated, especially when the true order is high. With a sample size of 300 , a threshold specified slightly less than the true separation probability may produce a good percentage of correct estimates. If the threshold specified is very different from the true separation probability, the correctly estimated percentage can be much lower, even close to zero. It is obviously important to know the threshold value even for large samples. Moreover, the above simulations also indicate that for a small sample size, even with a correctly specified threshold there may still be limited capability to obtain a high accuracy in estimating the exact order. Again, small sample sizes impose serious limitations on the performance of order estimation. Based on the small sample sizes that occur with microarray data, the information for discovering the underlying order is too limited. This is the reason that we suggest 
identifying the response genes with order above 1 for microarray data rather than estimating the exact order.

In the simulations above, the data were generated from the mixture density (2.1). To evaluate the performance of our method when the observations are generated from hidden Markov models, we generate data from a homogeneous hidden Markov model with two states. As shown in Table 5.6, we evaluated the HMMs with two gamma emission distributions. These two emission distributions are specified in the same way as in Table 5.4. The stationary distribution is $(0.4,0.6)$ for the two states corresponding to the two gamma emission distributions in Table 5.6. A homogeneous Markov chain is assumed, with transition matrix: $A=\left[\begin{array}{ll}0.7 & 0.3 \\ 0.2 & 0.8\end{array}\right]$. The total number of time points is 5 .

For sample size of 20 or 300 , we generated 4 or 60 runs of observations respectively with five observations in each run. This data generation procedure is repeated 500 times to get the 500 samples for each row of Table 5.6. Our algorithm was then run on the 500 samples to estimate the order for each row, as shown in Table 5.6. The order estimation proportions are recorded in Table 5.6. Comparing the result between Table 5.4 and Table 5.6, the performance of our algorithm is similar for data generated from finite mixture models and homogeneous hidden Markov models. Our simulation results also provide certain support to the suggestion proposed by Poskitt and Zhang (2005). 
Table 5.6. Simulation evaluations for hidden Markov models with 2 hidden states. The sample size, true order, separation threshold, the true separation probability between the two emission distribution and the parameters of the two emission distributions are specified in following table.

\begin{tabular}{|c|c|c|c|c|c|c|c|c|c|}
\hline $\begin{array}{l}\text { Sample } \\
\text { size }\end{array}$ & $\begin{array}{l}\text { True } \\
\text { order }\end{array}$ & $\begin{array}{c}\text { Sep. } \\
\text { Threshold }\end{array}$ & $\begin{array}{l}\text { Sep. } \\
\text { prob. }\end{array}$ & $\begin{array}{l}\text { Initial } \\
\text { Transition } \\
\text { Dist. }\end{array}$ & $\begin{array}{c}\text { First } \\
\text { gamma } \\
\text { Dist. } \\
\text { (shape } \alpha, \\
\text { scale } \beta \text { ) }\end{array}$ & $\begin{array}{l}\text { Second } \\
\text { gamma Dist. } \\
\text { (shape } \alpha, \\
\text { scale } \beta \text { ) }\end{array}$ & $\begin{array}{c}\text { Among } \\
500 \\
\text { repeats, } \\
\text { the } \\
\text { proportion } \\
\text { of order }<2 \\
\end{array}$ & $\begin{array}{l}\text { Among } 500 \\
\text { repeats, } \\
\text { the } \\
\text { proportion } \\
\text { of order }=2\end{array}$ & $\begin{array}{l}\text { Among } 500 \\
\text { repeats, } \\
\text { the } \\
\text { proportion } \\
\text { of order }>2\end{array}$ \\
\hline 20 & 2 & $60 \%$ & $72 \%$ & $0.4,0.6$ & $(40,15)$ & $(40,17.85)$ & 0.282 & 0.546 & 0.172 \\
\hline 20 & 2 & $62 \%$ & $82 \%$ & $0.4,0.6$ & $(40,15)$ & $(40,19.8)$ & 0.098 & 0.566 & 0.336 \\
\hline 20 & 2 & $68 \%$ & $92 \%$ & $0.4,0.6$ & $(40,15)$ & $(40,23.1)$ & 0.06 & 0.564 & 0.376 \\
\hline 20 & 2 & $63 \%$ & $73 \%$ & $0.4,0.6$ & $(20,15)$ & $(20,19.2)$ & 0.29 & 0.506 & 0.204 \\
\hline 20 & 2 & $63 \%$ & $\begin{array}{c}83 \% \\
.\end{array}$ & $0.4,0.6$ & $(20,15)$ & $\begin{array}{c}(20, \\
22.35)\end{array}$ & 0.13 & 0.64 & 0.23 \\
\hline 20 & 2 & $63 \%$ & $93 \%$ & $0.4,0.6$ & $(20,15)$ & $(20,28.2)$ & 0.014 & 0.572 & 0.414 \\
\hline 20 & 2 & $66 \%$ & $76 \%$ & $0.4,0.6$ & $(10,15)$ & $(10,22.05)$ & 0.35 & 0.53 & 0.12 \\
\hline 20 & 2 & $66 \%$ & $86 \%$ & $0.4,0.6$ & $(10,15)$ & $(10,28.05)$ & 0.096 & 0.622 & 0.282 \\
\hline 20 & 2 & $66 \%$ & $96 \%$ & $0.4,0.6$ & $(10,15)$ & $(10,43.05)$ & 0.004 & 0.51 & 0.486 \\
\hline 20 & 2 & $69 \%$ & $79 \%$ & $0.4,0.6$ & $(5,15)$ & $(5,27.15)$ & 0.37 & 0.50 & 0.13 \\
\hline 20 & 2 & $69 \%$ & $89 \%$ & $0.4,0.6$ & $(5,15)$ & $(5,39.75)$ & 0.084 & 0.57 & 0.346 \\
\hline 20 & 2 & $69 \%$ & $99 \%$ & $0.4,0.6$ & $(5,15)$ & $(5,112.05)$ & 0 & 0.41 & 0.59 \\
\hline 300 & 2 & $66 \%$ & $72 \%$ & $0.4,0.6$ & $(40,15)$ & $(40,17.85)$ & 0.166 & 0.756 & 0.078 \\
\hline 300 & 2 & $70 \%$ & $82 \%$ & $0.4,0.6$ & $(40,15)$ & $(40,19.8)$ & 0.122 & 0.68 & 0.198 \\
\hline 300 & 2 & $76 \%$ & $92 \%$ & $0.4,0.6$ & $(40,15)$ & $(40,23.1)$ & 0.082 & 0.73 & 0.188 \\
\hline 300 & 2 & $66 \%$ & $73 \%$ & $0.4,0.6$ & $(20,15)$ & $(20,19.2)$ & 0.13 & 0.686 & 0.184 \\
\hline 300 & 2 & $71 \%$ & $83 \%$ & $0.4,0.6$ & $(20,15)$ & $\begin{array}{c}(20, \\
22.35)\end{array}$ & 0.124 & 0.68 & 0.196 \\
\hline 300 & 2 & $76 \%$ & $93 \%$ & $0.4,0.6$ & $(20,15)$ & $(20,28.2)$ & 0.004 & 0.718 & 0.278 \\
\hline 300 & 2 & $70 \%$ & $76 \%$ & $0.4,0.6$ & $(10,15)$ & $(10,22.05)$ & 0.1 & 0.716 & 0.184 \\
\hline
\end{tabular}




\begin{tabular}{|c|c|c|c|c|c|c|c|c|c|}
\hline 300 & 2 & $74 \%$ & $86 \%$ & $0.4,0.6$ & $(10,15)$ & $(10,28.05)$ & 0.194 & 0.584 & 0.222 \\
\hline 300 & 2 & $79 \%$ & $96 \%$ & $0.4,0.6$ & $(10,15)$ & $(10,43.05)$ & 0 & 0.706 & 0.294 \\
\hline 300 & 2 & $74 \%$ & $79 \%$ & $0.4,0.6$ & $(5,15)$ & $(5,27.15)$ & 0.2 & 0.62 & 0.18 \\
\hline 300 & 2 & $77 \%$ & $89 \%$ & $0.4,0.6$ & $(5,15)$ & $(5,39.75)$ & 0.162 & 0.582 & 0.256 \\
\hline 300 & 2 & $82 \%$ & $99 \%$ & $0.4,0.6$ & $(5,15)$ & $(5,112.05)$ & 0 & 0.766 & 0.234 \\
\hline
\end{tabular}




\section{Chapter 6}

\section{ANALYSIS OF POLYMORPHONUCLEAR LEUKOCYTE MICROARRAY TIME SERIES DATA}

In this chapter, the methods presented in chapter 3 and 4 are applied to a microarray time course data set which is available on the NCBI GEO web site (http://www.ncbi.nlm.nih.gov/geo/). This data set is published on GEO with the data set ID of GDS1428 (Borjesson et al., 2005). The experiment uses Affymetrix oligonucleotide arrays. It is designed to investigate the effect of infection of A. phagocytophilum on polymorphonuclear leukocytes (PMNs or netrophils). We choose to analyze two of the treatment conditions from this experiment: PMNs treated with A.phagocytophilum, and PMNs treated by the control condition (i.e. without any infection). There are seven time points with 2 to 6 replicates at each time point, under each treatment condition. The intensity values analyzed are the normalized values computed using GeneSpring version 6.0 software. For further details, see Borjesson et al. (2005). 
The data are analyzed using the methods of chapter 3 and 4, for each treatment condition separately. We use gamma distributions to model the expression intensities at each time point for each gene. For the GDS1428 data set, the normalized intensity measurements after pre-processing are used as the input data for all subsequent analysis. As described in chapter 3, empirical Bayesian analysis is first performed to find the gene specific shape parameter. This shape parameter and the CV are computed for each gene. These values are assumed to be the same across time points for one specific gene. The histograms for the estimated CV and shape parameter values are plotted in Figure 6.1 and Figure 6.2.
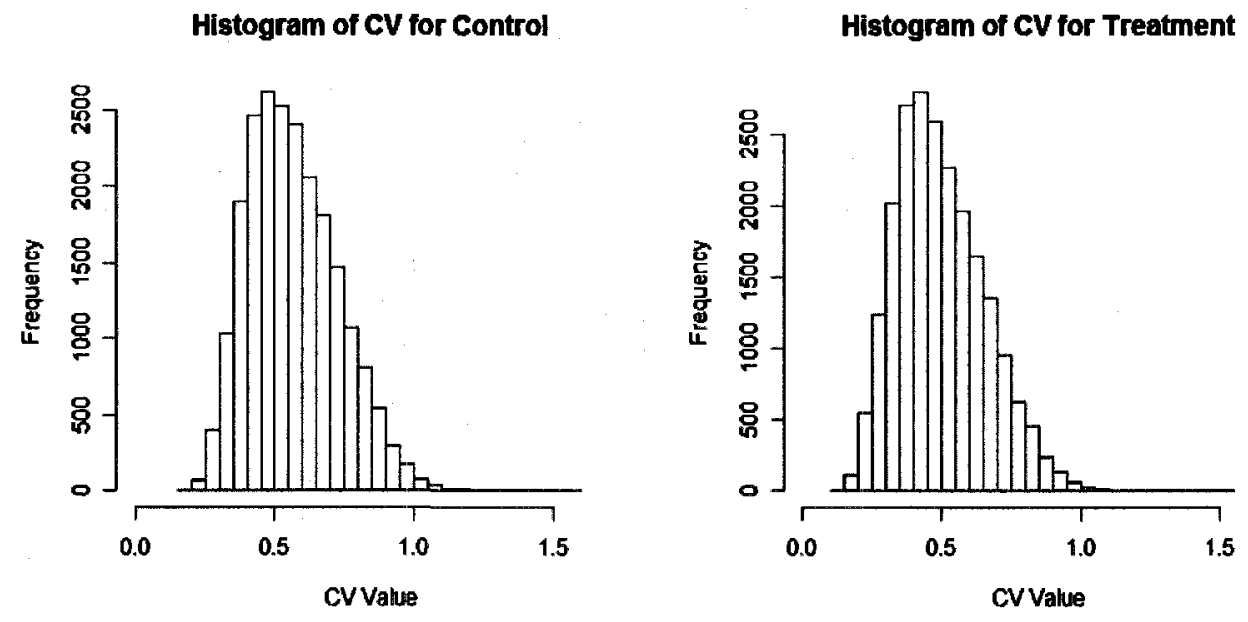

Figure 6.1 Histogram of CV values for control and treatment condition of GDS1428 $(n=21775)$. 

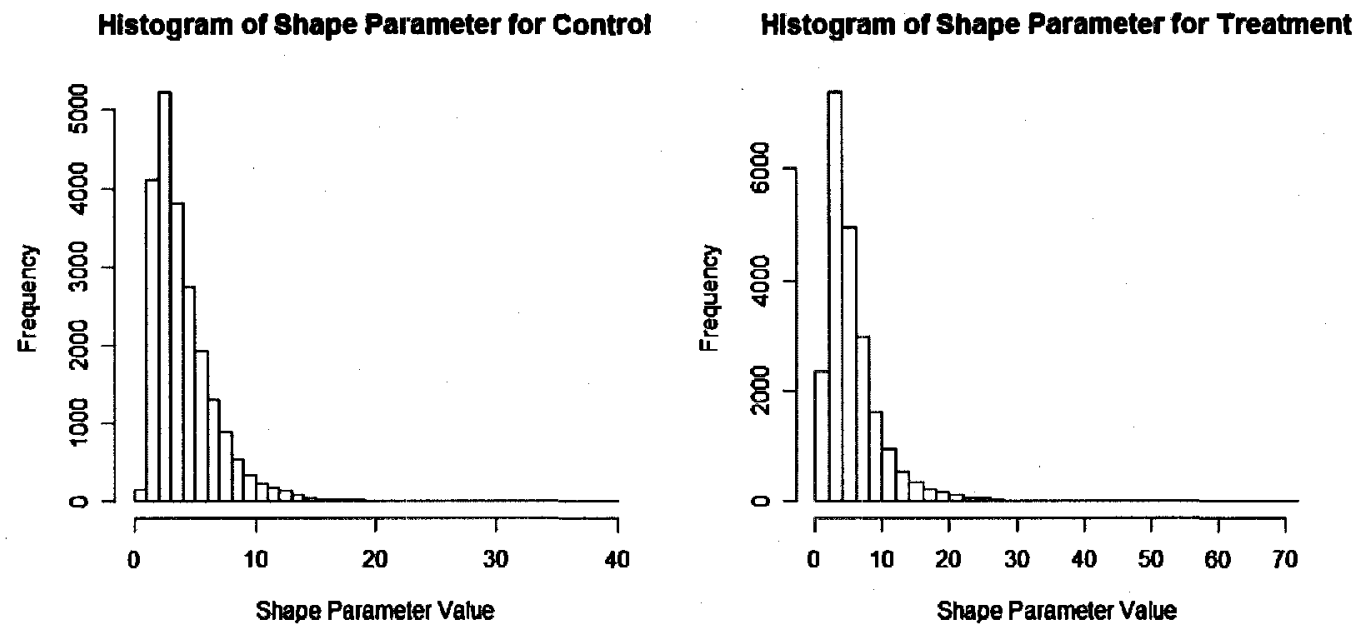

Figure 6.2 Histogram of shape parameter values for control and treatment condition of GDS1428 $(n=21775)$.

As we discussed in chapter 3, the shape parameters of all genes under the same treatment condition are generated from a log-normal prior distribution. If we think of the $\mathrm{CV}$ as a unitless measurement of variation, the shape parameter of the gamma distribution can be thought of as the squared stability measurement for a specific gene. The estimated hyper-parameters using (3.2) and (3.3) for the two treatment conditions are: $\hat{\tau}: 2.182803$ and $\hat{\sigma}: 1.955049$ for the GDS1428 control group; and $\hat{\tau}: 2.295019$ and $\hat{\sigma}: 1.840149$ for the GDS1428 treatment group. These histograms suggest that the assumption made here and by Lo and Gottardo (2007) that the shape parameter follows a lognormal distribution is a reasonable claim. The shape parameter is estimated based on the posterior distribution (3.7) for each gene.

With the shape parameter estimated and treated as known in later stage analysis, the order is estimated by the penalized maximum likelihood method as discussed in 
chapter 4 . The initial upper bound for order is set to 5, with corresponding starting mixing proportions set to 0.2 for each of the five components. The separation threshold values chosen are those identified in chapter 5 , as the values with stars shown in table 5.2. These separation threshold values are used to control the type I error rate at about $3 \%$, corresponding to different shape and scale parameter values. Choosing a $3 \%$ type I error rate level here is only for illustration purposes. From the analysis of several other microarray time course data sets (results not shown), when the threshold value is above 0.9 , there is usually no gene showing more than one state. This suggests that genes do not use very high separation probabilities to increase signal certainty. Perhaps the biological cost is prohibitively high for statistically significant separation. Instead, implementing some check points across cell growth stages may be more cost effective.

For the GDS1428 data set, we found 1644 response genes which responded under the control treatment condition, and 1902 genes which responded under the treatment condition. As shown in Table 6.1, the total number of genes measured in both the control and treatment microarray is 13996 . The number of genes which did not respond under the

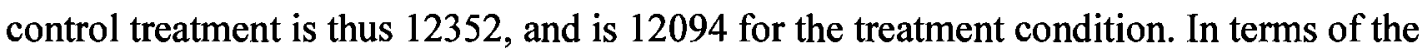
percentage of response genes, $11.75 \%$ and $13.59 \%$ of genes respond under the control and treatment conditions, respectively. After the 3\% type I error rate adjustment, the expected response gene percentages are $11.40 \%$ and $13.18 \%$ for control and treatment groups, respectively. The total number of probes are 21775 for both control and treatment conditions. According to the Affymetrix gene chip design, normally more than one probe is used for each gene, making the number of probes bigger than the number of genes. Our discovered response genes are annotated first based on their probe ID. 
Table 6.1. The number of response genes for each treatment condition for the GDS1428 data.

\begin{tabular}{|c|c|c|}
\hline & GDS1428 Control & GDS1428 Treatment \\
\hline Number of Response Genes & 1644 & 1902 \\
\hline $\begin{array}{c}\text { Number of non-Response } \\
\text { Genes }\end{array}$ & 12352 & 12094 \\
\hline Total Number of Probes & 21775 & 21775 \\
\hline Total Number of Genes & 13996 & 13996 \\
\hline
\end{tabular}

In Table 6.1, the annotation platform of hgu133A from Affymetrix is used. The probe ID is first used to identify the response genes. Then based on Affymetrix's annotation database, the corresponding gene ID is identified. Since there are typically several probe ID's matching one gene ID, the number of probes is bigger than the number of gene ID. We choose to report gene ID in Table 6.1 and in the tables of Appendix A for ease of biological interpretation. The full list of the response genes under one treatment condition is the summary of cellular response under one external condition. The information maintained in each response set is not only about the appearance of a single gene but also about the co-appearance with other genes in that set. Thus, the full response set as a whole provides a signature for the cellular response.

The sets of response genes are presented in Table A.1 and Table A.2 of Appendix A. There are 894 genes which are responded under both the control and treatment conditions. This common set of genes is listed in Table A.3 of Appendix A. The genes that responded only for the control group are listed in Table A.4, and the genes which 
responded only under the treatment condition are listed in Table A.5 of Appendix A. It is biologically reasonable that certain genes are commonly used by a cell to respond to different external conditions. This situation is similar to those house keeping genes which are activated across different treatment conditions.

The comparison of the response set of genes under different treatment conditions can provide us with more information regarding regulatory relationships among the genes. And as the number of gene sets extracted from experiments increases, the relationship inferred can be more reliable. We identify the commonly activated gene set for the GDS1428 treatment and control group. When many treatment conditions are compared, if we find one gene's appearance has a high probability to co-appear with another gene, they are probably involved in the same or a closely related regulatory pathway. As an example, as shown in Table A.3, among the common response gene set for both control and treatment conditions, we find genes: NFKB1, NFKB2, NFKBIE, EIF2AK3. Current biological knowledge indicates that NFKB1 and NFKB2 may both respond under various treatment conditions. This is confirmed by our result. NFKB1 and NFKB2 encode for the nuclear factor dimmer protein that regulates the transcription of genes involved in immune and inflammatory responses, stress remediation, cell growth and apoptosis. In normal situations, the protein encoded by the NFKB1 and NFKB2 genes are associated with the inhibitor (IKB) and only presented in cytoplasm. In response to certain conditions, IKB will be degraded and will release NFKB1 and NFKB2 proteins into the nucleus and induce a series of gene activations. 
There is a complicated mechanism that controls the release of NFKB proteins and different activation processes when they enter the nucleus. The IKK (IKB kinase) is one kinase that contributes to the degradation and the release of IKB from NFKB proteins. We found that NFKB1 and NFKB2 genes responded under both the control and treatment conditions. In addition, NFKBIE (or IKBE) also responds under both conditions, which means the proteins of NFKB1 or NFKB2 are inhibited by IKBE and kept in the cytoplasm under both conditions. On the other hand, the gene IKBKAP responds only in the control group. This gene encodes a scaffold protein which assists to form IKKs into an active kinase complex, which in turn may lead to the release of NFKB proteins into the nucleus.

The gene EIF2AK3 responds under both the control and treatment conditions. This gene is related to the reaction of impaired protein folding in the endoplasmic reticulum (i.e. ER stress). ER stress is one of several mechanisms involving NFKB mediated regulation. Phosphorylation of another translation related protein by EIF2AK3 results in general reduction of translation, which allows the cell more time to correct the impaired protein folding problem caused by ER stress. In the mean time, the phosphorylation also contributes to the activation of NFKB protein by a mechanism that involves release, but not degradation of IKB. Both response sets have the co-occurrence of NFKB1, NFKB2 and EIF2AK3, indicating that they probably work together in activation of the pathway for ER stress. For more information, please see Jiang et al. (2003) and Liang et al. (2006). 
However, the common response set of 894 genes we found for both control and treatment conditions does not necessarily indicate a deterministic causal relationship between any pair of the genes in this set. Genes in this set may belong to several parallel pathways within which the casual relationship is found to be more significant. Moreover, even within the same pathway, the regulatory relationship is still governed by probabilistic rules. If two genes always appear together under many experimental conditions, the probability of the causal relationship among them is much higher than that under a few experimental conditions. Hence, the statistical inference of the causal relationship among genes can be dramatically improved as the number of experiments analyzed increases.

To illustrate the difference between our approach and the direct expression comparion approaches, we compare our response gene lists with the differentially regulated gene list identified in the original study (Borjesson et al., 2005). Those genes identified by Borjesson et al. are defined as differentially regulated genes, as listed in Table 1 of their complementary material (Borjesson et al., 2005). Their approach compares the gene expression levels between the treatment and the control group. Those genes with at least 1.5 fold changes over the control group are defined as differentially regulated genes (including both up-regulated genes and down-regulated genes). The gene expression comparisons were conducted between treatment and control at the time points 1.5 hours, 3 hours, 6 hours, 9 hours, 12 hours and 24 hours. To make the comparison of the gene lists from both studies possible, the overall differentially expressed gene list from their study is used. This gene list is referred to as gene set A hereafter. Gene set A consists of those genes with 1.5 or higher fold changes at one or more time points 
between treatment and control group. The total number of these genes in gene set A is 810 , which is the number of unique gene IDs with at least one corresponding probe set found to meet the criterion. Because the response gene lists in our study are defined differently from theirs, we use two of the most relevant gene sets in the comparison. The first one is the response gene set found under the treatment condition, which is referred to as gene set B hereafter. Gene set B has 1902 response genes. The second gene list consists of those genes found to respond only under the treatment condition, which is referred to as gene set $\mathrm{C}$. Gene set $\mathrm{C}$ has 1008 genes. The difference between gene set $\mathrm{B}$ and $\mathrm{C}$ is that those genes found to respond under the control condition are excluded from gene set $\mathrm{C}$.

The comparison is shown in Table 6.2. There are 232 genes found in both sets $\mathrm{A}$ and B. 578 genes are in gene set A but not B. There are 1670 genes identified only in gene set $\mathrm{B}$. For gene set $\mathrm{A}$ and $\mathrm{C}$, there are 80 genes in common. 730 genes appear only in gene set $A$ and 928 genes are identified only in gene set $C$. 
Table 6.2. The comparison of gene lists identified by our proposed method and the method described in the original study (Borjesson et al., 2005). Gene Set A contains the differentially regulated gene set based on the comparison between treatment and control condition as defined in the original study. Gene Set B is the response gene set under treatment condition. Gene Set $\mathrm{C}$ contains the genes which respond only under treatment condition. Gene Set B and C are found using our proposed method.

\begin{tabular}{|c|c|c|c|}
\hline \multirow{2}{*}{} & \multicolumn{3}{|c|}{$\begin{array}{r}\text { Gene Set A: Differentially Regulated Genes Between } \\
\text { Treatment and Control, total 810 genes }\end{array}$} \\
\cline { 2 - 4 } & $\begin{array}{c}\text { Number of genes } \\
\text { in both set }\end{array}$ & $\begin{array}{c}\text { Number of genes } \\
\text { in Set A only }\end{array}$ & $\begin{array}{c}\text { Number of genes } \\
\text { in Set B/C only }\end{array}$ \\
\hline $\begin{array}{c}\text { Gene Set B: Response } \\
\text { Genes Under Treatment, } \\
\text { total 1902 genes }\end{array}$ & 232 & 578 & 1670 \\
\hline $\begin{array}{c}\text { Gene Set C: Response Gene } \\
\text { Under Treatment Only, total } \\
1008 \text { genes }\end{array}$ & 80 & 730 & 928 \\
\hline
\end{tabular}

As shown in the above comparison, the two approaches are quite different in terms of their identified gene list. This is probably due to the difference in analysis methods used and the definition of the targeted genes. Detailed gene lists for the above comparisons are included in Table A. 6 to Table A.11 of Appendix A. 


\section{Chapter 7}

\section{CONCLUSIONS AND FURTHER DISCUSSION}

We introduce a method based on order estimation to deal with the confounding problem in microarray time course data. Because the treatment effect is confounded with the gene context effect, the commonly used statistical comparisons and tests are inappropriate. The fact that a treatment condition can only be administrated to the cell level and that it affects different genes in a gene specific way requires more attention to the analysis. The influence of a treatment condition on a gene potentially depends on other genes in a way which is unknown and could not be simply ignored. In such a situation, the ANOVA models and similar linear models incorporating a correlation structure are not sufficient. Results obtained from this kind of analysis, especially those aimed at identifying genes with differential expressions, are incorrect. Because the regulatory relationship is not deterministic, the expressions of a specific gene under the same treatment condition do not necessary come from the same underlying state each 
time. The comparison of means in such a situation can not provide conclusive information on whether the gene behaves the same or not. For the same reason, the comparison of the state sequence between two treatment conditions can not provide information about whether the gene behaves the same or not either. Since the comparison for non-time course experiments is only a special form of time course experiments with a single time point, the most direct informative statistic under this situation is the order, which provides the essential information about the differentiation of states instead of a particular state. The differentiation in expressions rather than the expression itself can be used to characterize the behavior of a gene for a treatment condition.

The method we proposed here is developed to first extract the transcription level response. The response genes are used by a cell either to communicate a signal to other genes or to perform certain biological functions. The identified response genes are expected to be those genes coordinated by the cell to react to a specific external condition. A cell response is much more complicated than a single gene response. This kind of response thus can be characterized by the full set of response genes.

The response genes are identified separately under each experimental condition. We developed the method to estimate the order when the mean and shape parameters are all unknown. Our penalized maximum likelihood estimation coupled with the simulation based approach to find the threshold provides a way to estimate the order, particularly suitable for microarray time course data. The sample size is typically small and there is no previous quantification for the separation probability. 
To apply our method, certain conditions are required to ensure proper inferences. Our approach requires the following conditions to be satisfied:

1. The time course experiments are synchronized.

2. There are replicates at each time point.

3. There are at least three time points measured.

The number of time points is very important because more time points provide better chance to detect response genes, if the different states do exist. The time intervals are not required to be equal, based on the stationary Markov process assumed for order estimation. The above conditions are satisfied for most microarray time course experiments.

To deal with the limited sample size of microarray data, we propose to target the separation between genes with order one and the genes with order two or above. Our method is designed specially for microarray time course data and takes into account the typical small sample size. One of the reasons that the order estimation is a difficult problem is that it is related to the sample size and the information available for the separation of components at a given sample size. This perspective has not been discussed much by the existing literature, which focuses on the asymptotic properties in the large sample situation.

Another issue of order estimation for HMMs is the assumption used for the transition probabilities. Interestingly, although existing frequentist methods always adopt the stationary probability assumption, there is no formal justification in the literature. Poskitt and Zhang (2005) provided some reasons for computational gain. Perhaps the 
order estimation for HMMs requires more clear definition. Before the order of a HMM is determined, there is no way to tell whether the underlying process is a homogeneous or heterogeneous. A different order may also change a homogenous process to a heterogeneous one. In other words, a sufficiently large order will always produce a homogeneous process. Without knowing the order, the states can not be defined. Without the definition of the states, we do not even have a criterion to judge whether a process as heterogeneous or homogeneous. The order estimation therefore should not be influenced by such a subjective decision. Hence a stationary probability can be reasonably assumed for the order estimation.

The identified response gene set as a characterization for a particular treatment condition has two fold meaning. First, each response gene plays a certain role in the response to a treatment condition. Second, the regulatory interaction with other genes is maintained in the response set by the gene's appearance together with other genes in the set. This co-appearance of genes in a response set provides information concerning the regulatory relationships among genes.

There are several possible further developments. For each gene, the shape parameter is assumed to be the same across the time points. This can be examined by performing statistical tests to find out whether it is the case for every gene. The shape parameter then can be estimated according to the test results. Theoretically it is possible to let the number of hidden states depend on both the shape and mean parameters. However, this modeling setting still needs supporting biological evidence, especially on whether the shape parameter changes its value across time. 
For hidden Markov models and finite mixture models, our order estimation method relies on the specification of the threshold. Finding a proper threshold value is critical and could be another possible further development. As gene expression data is accumulated, the knowledge about the separation probability for different genes may become available and can be used in further analysis of new time course data. However, as we begin our proposed analysis, this kind of information is very difficult to find. Thus our proposed simulation based approach provides a way to start. There is certainly much room for further improvement. 


\section{References}

Albert, P. S., McFarland, H. F., Smith, M. E., and Frank, J. A. (1994). Time series for modeling counts from a relapsing-remitting disease: application to modeling disease activity in multiple sclerosis. Statistics in Medicine 13, 453-466.

Aitkin, M. and Rubin, D. B. (1985). Estimation and hypothesis testing in finite mixture models. Journal of the Royal Statistical Society, Series B 47, 67-75.

Baggerly, K. A., Coombes, K. R., Hess, K. R., Stivers, D. N., Abruzzo, L. V., and Zhang, W. (2001). Identifying differentially expressed genes in cDNA microarray experiments. Journal of Computational Biology 8, 639-659.

Baldi, P. and Long, A. D. (2001). A Bayesian framework for the analysis of microarray expression data: regularized t-test and statistical inference of gene changes.

Bioinformatics 17, 509-519.

Bar-Joseph, Z., Gerber, G., Simon, I., Gifford, D., and Jaakkola, T. (2003). Comparing the continuous representation of time-series expression profiles to identify differentially expressed genes. Proceedings of the National Academy of Sciences 100, 10146-10151. 
Baras, J. S. and Finesso, L. (1992). Consistent estimation of the order of hidden Markov chains. Stochastic Theory and Adaptive Control: Proceedings of a Workshop held in Lawrence, Kansas, September 26-28, 1991. Berlin: Springer-Verlag.

Bickel, P. J., Ritov, Y., and Ryden, T. (1998). Asymptotic normality of the maximumlikelihood estimator for general hidden Markov models. Annals of Statistics 26, 16141635.

Biernacki, C., Celeux, G., and Govaert, G. (1998). Assessing a mixture model for clustering with the integrated classification likelihood. Technical Report No. 3521. Rhone-Aples: INRIA.

Black, M. A. and Doerge, R. W. (2001). Calculation of the minimum number of replicates spots required for detection of significant gene expression fold change in microarray experiments. In Proceeding of the Conference on Applied Statistics in Agriculture 144-158.

Bolstad, B., Irizarry, R., Astrand, M., and Speed, T. (2002). A comparison of normalization methods for high density oligonucleotide array data based on variance and bias. Technical report, UC Berkeley.

Borjesson, D. L., Kobayashi, S. D., Whitney, A. R., Voyich, J. M., Argue, C. M., and DeLeo, F. R. (2005). Insights into pathogen immune evasion mechanisms: anaplasma 
phagocytophilum fails to induce an apoptosis differentiation program in human neutrophils. The Journal of Immunology 174, 6364-6372.

Calvano, S. E., Xiao, W., Richards, D. R., Felciano, R. M., Baker, H. V., Cho, R. J., Chen, R. O., Brownstein, B. H., Cobb, J. P., Tschoeke, S. K., Miller-Graziano, C., Moldawer, L. L., Mindrinos, M. N., Davis, R. W., Tompkins, R. G., and Lowry, S. F. (2005). A network-based analysis of systemic inflammation in humans. Nature 437, 1032-1037.

Cappe, O., Moulines, E., and Ryden, T. (2005). Inference in Hidden Markov Models. USA: Springer.

Chen, J. and Kalbfeisch, J. D. (1996). Penalized minimum-distance estimates in finite mixture models. The Canadian Journal of Statistics 24, 167-175.

Chen, Y., Dougherty, E. R., and Bittener, M. L. (1997). Ratio-based decisions and the quantitative analysis of cDNA microarray images. Journal of Biomedical Optics 2, 364374.

Chen, H., Chen, J., and Kalbfleisch, J. D. (2001). A modified likelihood ratio test for homogeneity in finite mixture models. Journal of the Royal Statistical Society, Series B 63, 19-29.

Chen, J. and Khalili, A. (2005). Order selection in finite mixture models. Technical report. Available at http://www.math.uwaterloo.ca/ jhchen/publ.html. 
Craig, B. A, Black, M. A.; and Doerge, R. W. (2003). Gene expression data: the technology and statistical analysis. Journal of Agricultural, Biological and Environmental Statistics 8, 1-28.

Craven, P. and Wahba, G. (1979). Smoothing noisy data with spline functions: estimating the correct degree of smoothing by the methods of generalized cross-validation, Numerische Mathematika 31, 377-403.

Csiszar, I. and Shields, P. C. (2000). The consistency of the BIC Markov order estimator. Annals of Statistics 28, 1601-1619.

Cwik, J. and Koronacki, J. (1997). A combined adaptive-mixtures estimator of multivariate probability densities. Computational Statistics and Data Analysis 26, 199218.

Dempster, A. P., Laird, N. M., and Rubin, D. B. (1977). Maximum likelihood from incomplete data via the EM algorithm (with discussion). Journal of the Royal Statistical Society, Series B 39, 1-38.

Dennis, B. and Patil, G. P. (1984). The gamma distribution and weighted multimodal gamma distributions as models of population abundance. Mathematical Biosciences 68 , 187-212. 
Dudoit, S., Fridlyand, J., and Speed, T. P. (2002). Comparison of discrimination methods for the classification of tumors using gene expression data. Journal of the American Statistical Association 97, 77-87.

Dudoit, S., Yang, Y. H., Callow, M. J., and Speed, T. P. (2000). Statistical methods for identifying differentially expressed genes in replicated cDNA microarray experiments. Technical Report 578, Statistics Department, University of California at Berkeley.

Durbin, B. P., Hardin, J. S., Hawkins, D. M., and Rocke, D. M. (2002). A variancestabilizing transformation for gene-expression microarray data. Bioinformatics 18, S105-S110.

Eisen, M., Spellman, P., Brown, P., and Botstein, D. (1998). Cluster analysis of genomewide expression patterns. Proceeding of the National Academy of Sciences 95, 1486314868.

Fan, J. and Li, R. (2001). Variable selection via non-concave penalized likelihood and its oracle properties. Journal of the Acoustical Society of America 96, 1348-1360.

Fan, J. and Li, R. (2002). Variable selection for Cox's proportional hazards model and frailty model. Annual of Statistics 30, 74-99.

Feng, Z. D. and McCulloch, C. E. (1996). Using bootstrap likelihood ratio in finite mixture models. Journal of the Royal Statistical Society, Series B 58, 609-617. 
Hansen, B. (1992). The likelihood ratio test under nonstandard conditions: testing the Markov switching model of GNP. Journal of Applied Econometrics 7,127-157, Erratum, (1996). 11, 195-198.

Hamilton, J. (1996). Specification testing in Markov switching time series models.

Journal

of Econometrics 70, 127-157.

Heckman, J. J., Robb, R. and Walker, J. R. (1990). Testing the mixture of exponentials hypothesis and estimating the mixing distribution by the method of moments. Journal of American Statistical Association 85, 582-589.

Hughes, J. P. and Guttorp, P. (1994). A class of stochastic models for relating synoptic atmospheric patterns to regional hydrologic phenomena. Water Resources Research 30,1535-1546.

Ideker, T., Thorsson, V., Siegel, A. F., and Hood, L. E. (2000). Testing for differentiallyexpressed genes by maximum-likelihood analysis of microarray data. Journal of Computational Biology 7, 805-817.

James, L. F., Priebe, C. E., and Marchette, D. J. (2001). Consistent estimation of mixture complexity. Annals of Statistics 29, 1281-1296. 
Jiang, H. Y., Wek, S. A., McGrath, B. C., Scheuner, D., Kaufman, R., Cavener, D. R., and Wek, R. C. (2003). Phosphorylation of the alpha subunit of eukaryotic initiation factor 2 is required for activation of NFKB in response to diverse cellular stresses. Molecular and Cellular Biology 23, 5651-5663.

Kendziorski, C. M., Newton, M. A., Lan, H., and Gould, M. N. (2003). On parametric empirical Bayes methods for comparing multiple groups using replicated gene expression profiles. Statistics in Medicine 22, 3899-3914.

Kerr, M. K., Martin, M., and Churchill, G. A. (2000). Analysis of variance for gene expression microarray data. Journal of Computational Biology 7, 819-837.

Lee, M. T., Kuo, F. C., Whitmore, G. A., and Sklar, J. (2000). Importance of replication in microarray gene expression studies: statistical methods and evidence from repetitive cDNA hybridization. Proceedings of the National Academy of Science 97, 9834-9839.

Leroux, B.G. (1992). Consistent estimation of a mixing distribution. Annals of Statistics 20, $1350-1360$.

Leroux, B. G. and Puterman, M. L. (1992). Maximum-penalized likelihood estimation for independent and Markov-dependent mixture models. Biometrics 48, 545-558.

Li, C. and Wong, W. H. (2001). Model-based analysis of oligonucleotide arrays: expression 
index computation and outlier detection. Proceedings of the National Academy of Science 98, 31-36.

Liang, G., Audas, T. E., Li, Y. Cockram, G. P., Dean, J. D., Martyn, A. C., Kokame, K., and Lu, R. (2006). Luman/CREB3 induces transcription of the endoplasmic reticulum (ER) stress response protein herp through an ER stress response element. Molecular and Cellular Biology 26, 7999-8010.

Lo, K. and Gottardo, K. (2007). Flexible empirical Bayes models for differential gene expression. Bioinformatics 23, 328-335.

Lockhart, D. J., Dong, H. L., Dyrne, M. C., Follettie, M. T., Gallo, M. V., Chee, M. S., Mittman, M., Wang, C. W., Kobayashi, M., Horton, H., and Brown, E. L. (1996). Expression monitoring by hybridization to high density oligonucleotide arrays. Nature Biotechnology 14, 1675-1680.

Luan, Y. and Li, H. (2004). Model-based methods for identifying periodically expressed genes based on time course microarray gene expression data. Bioinformatics 20, 332-339.

MacDonald, I.L. and Zucchini, W. (1997). Hidden Markov Models and Other Models for Discrete-Valued Time Series. London: Chapman \& Hall.

MacKay, R. J. (2002). Estimating the order of a hidden Markov model. The Canadian Journal of Statistics 30, 573-589. 
McLachlan, G., and Peel, D. (2000). Finite Mixture Models. New York, Chichester, Weinheim, Brisbane, Singapore, Toronto: John Wiley \& Sons, Inc.

Nadon, R. and Shoemaker, J. (2002), Statistical issues with microarrays: processing and analysis. Trends in Genetics 18, 265-271.

Newton, M. A., Kendziorski, C. M., Richmond, C. S., Blattner, F. R., and Tsui, K. W. (2001). On differential variability of expression ratios: improving statistical inference about gene expression changes from microarray data. Journal of Computational Biology 8, 37-52.

Newton, M. A., Noueiry, A., Sarkar, D., and Ahlquist, P. (2004). Detecting differential gene expression with a semi-parametric hierarchical mixture method. Biostatistics 5, 155-176.

Nguyen, D., Bulak, A., Naisyin, W., and Carroll, R. (2002). DNA microarray experiments: biological and technological aspects. Biometrics 58, 701-717.

Park, T., Yi, S. G., Lee, S., Lee, S. Y., Yoo, D. H., Ahn, J. I., and Lee, Y. S. (2003). Statistical tests for identifying differentially expressed genes in time-course microarray experiments. Bioinformatics 19, 694-703. 
Poskitt, D. S. and Zhang, J. (2005). Estimating components in finite mixtures and hidden Markov models. Australian and New Zealand Journal of Statistics 47, 269-286.

Rabiner, L. R. (1989). A Tutorial on Hidden Markov Models and Selected Applications in Speech Recognition. Proceedings of the IEEE 77, 257-286.

Richardson, S. and Green, P. J. (1997). On Bayesian analysis of mixtures with an unknown number of components. Journal of the Royal Statistical Society, Series B 59, 731-792.

Richmond, C. S., Glasner, J. D. Mau, R., Jin, H., and Blattner, F. R. (1997). Genomewide expression profiling in Escherichia coli K-12. Nucleic Acids Research 27, 38213835 .

Rocke, D. M. and Durbin, B. (2001). A model for measurement error for gene expression arrays. Journal of Computational Biology 8, 557-570.

Roeder, K. and Wasserman, L. (1997). Practical density estimation using mixtures of normals. Journal of the American Statistical Association 92, 894-902.

Ryden, T. (1995). Estimating the order of hidden Markov models. Statistics 26, 345-354.

Schadt, E. E., Li, C., Su, C., and Wang, W. H. (2000). Analyzing high-density oligonucleotide gene expression array data. Journal of Cellular Biochemistry 80, 192-202. 
Schena, M., Shalon, D., Davis, R. W., and Brown, P. O., (1995). Quantitative monitoring of gene expression patterns with a complementary DNA microarray. Science 270, $467-$ 470.

Schliep, A., Schonhuth, A., and Steinhoff, C. (2003). Using hidden Markov models to analyze gene expression time course data. Bioinformatics 19, i255-i263.

Schliep, A., Steinhoff, C., and Schonhuth, A. (2004). Robust inference of groups in gene expression time-course using mixtures of HMMs. Bioinfomatics 20, i283-i289.

Schliep, A., Costa, I. G., Steinhoff, C., and Schonhuth, A. (2005). Analyzing gene expression time-courses. IEEE/ACM Transactions on Computational Biology and Bioinformatics 2, 179-193.

Snustad, D. P. and Simmons, M. J. (2003), Principles of Genetics (3rd edition). John Wiley \& Son, Inc.

Solka, J. L., Wegman, E. J., Priebe, C. E., Poston, W. L., and Rogers, W. (1998). Mixture structure analysis using the Akaike criterion and the bootstrap. Statistics and Computing $8,177-188$.

Stone, M. (1974). Cross-validatory choice and assessment of statistical predictions (With discussion). Journal of the Royal Statistical Society, Series B 36, 111-147. 
Tai, Y. C. and Speed, T. (2006). A multivariate empirical Bayes statistic for replicated microarray time course data. Annals of Statistics 34, 2387-2412.

Theilhaber, J., Bushnell, S., Jackson, A., and Fuchs, R. (2001). Bayesian estimation of fold-changes in the analysis of gene expression: the pfold algorithm. Journal of Computational Biology 8, 585-614.

Tibshirani, R. (1996). Regression shrinkage and selection via the lasso. Journal of the Royal Statistical Society, Series B 58, 267-288.

Tsodikov, A., Szabo, A., and Jones, D. (2002). Adjustments and tests for differential expression with microarray data. Bioinformatics 18, 251-260.

Yang, Y. H., Buckley, M. J., Dudoit, S., and Speed, T. P. (2000). Comparison of methods for image analysis on cDNA microarray data. Technical Report 584, Statistics Department, University of California at Berkeley.

Yuan, M. and Kendziorski, C. (2006). Hidden Markov models for microarray time course data in multiple biological conditions. Journal of the American Statistical Association $101,1323-1332$.

Wang, P. and Puterman, M. L. (1999). Markov Poisson regression models for discrete time series. Journal of Applied Statistics 26, 855-869. 


\section{Appendix A. Response Gene Tables.}

Table A.1. Response gene set for GDS1428 control group. Total 1644 response genes.

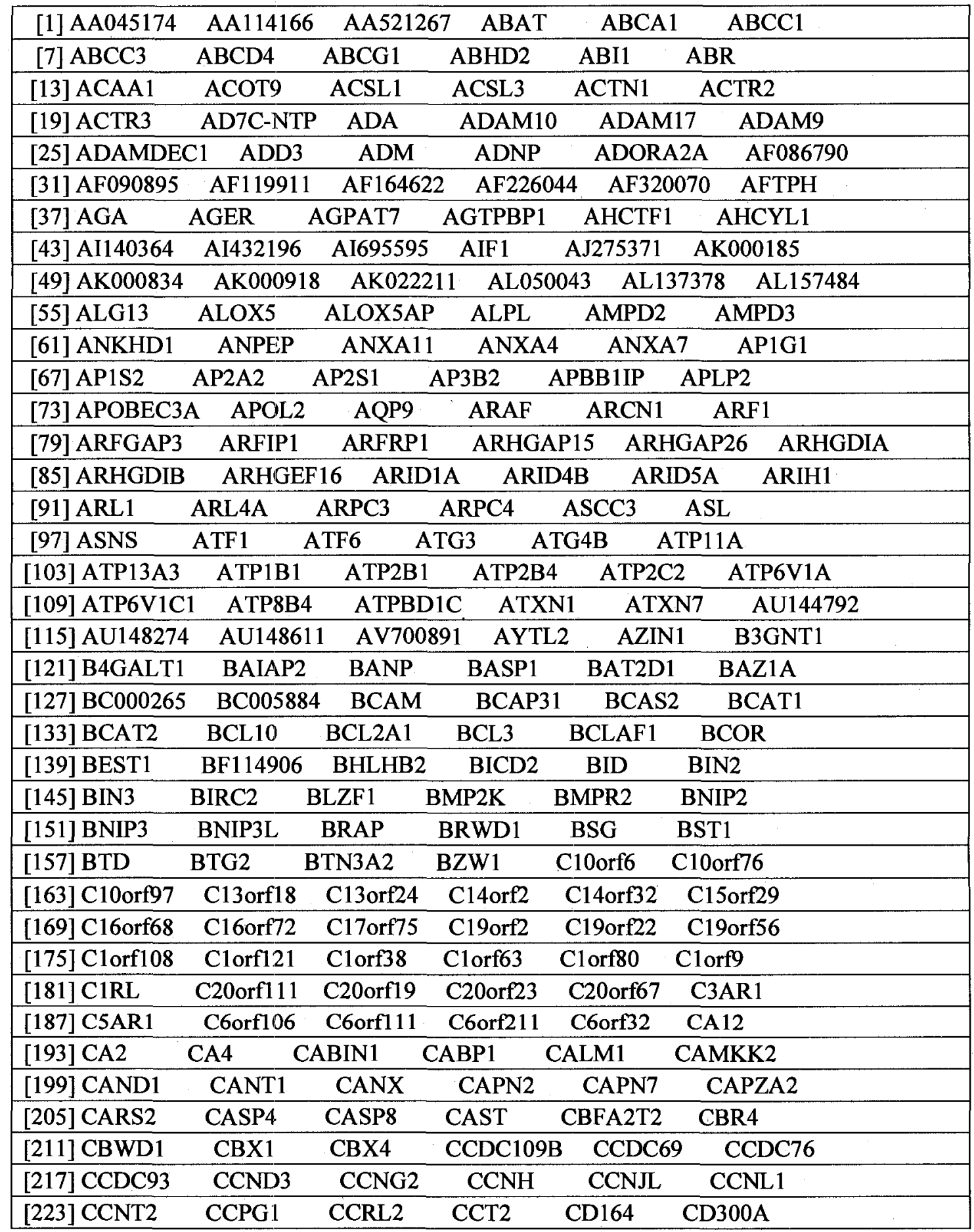




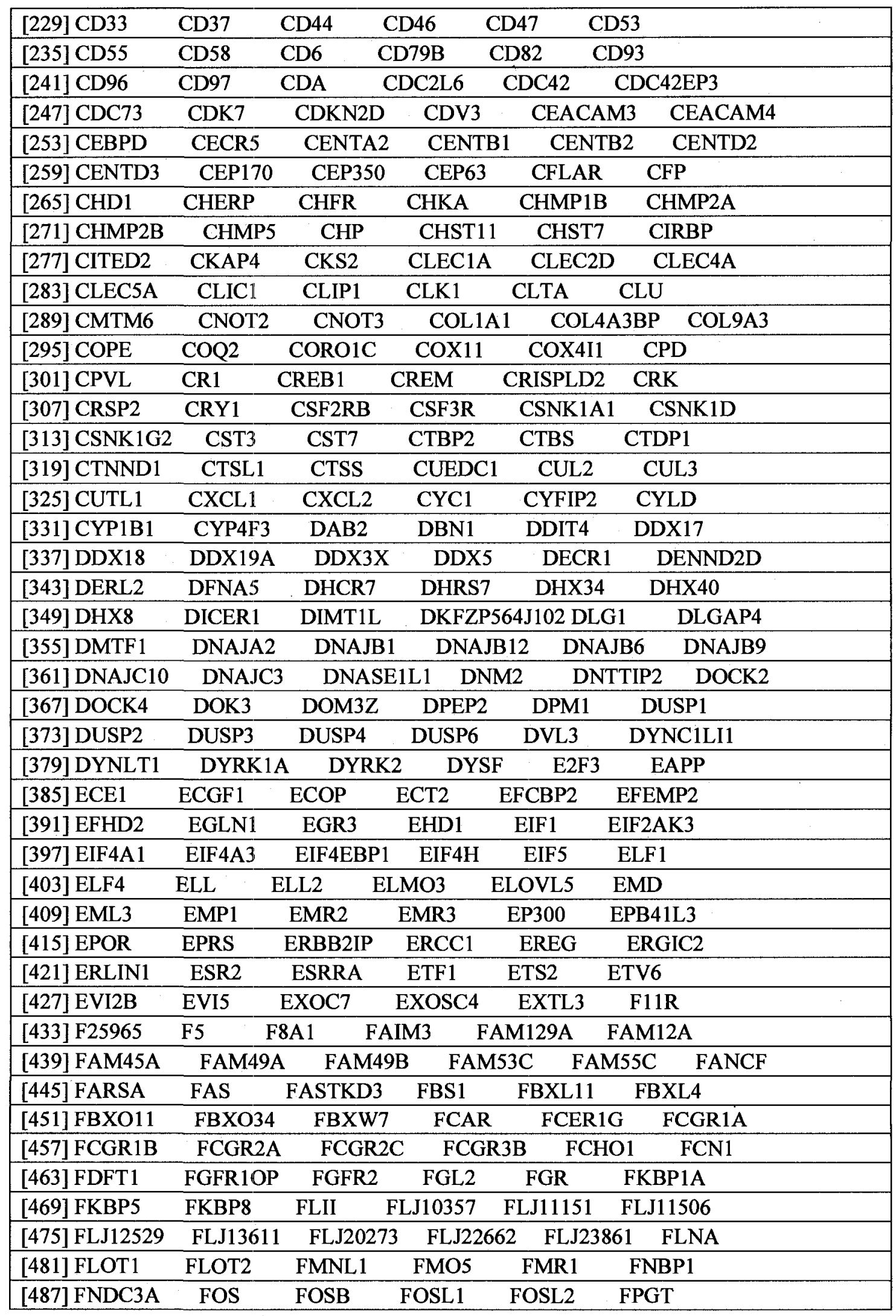




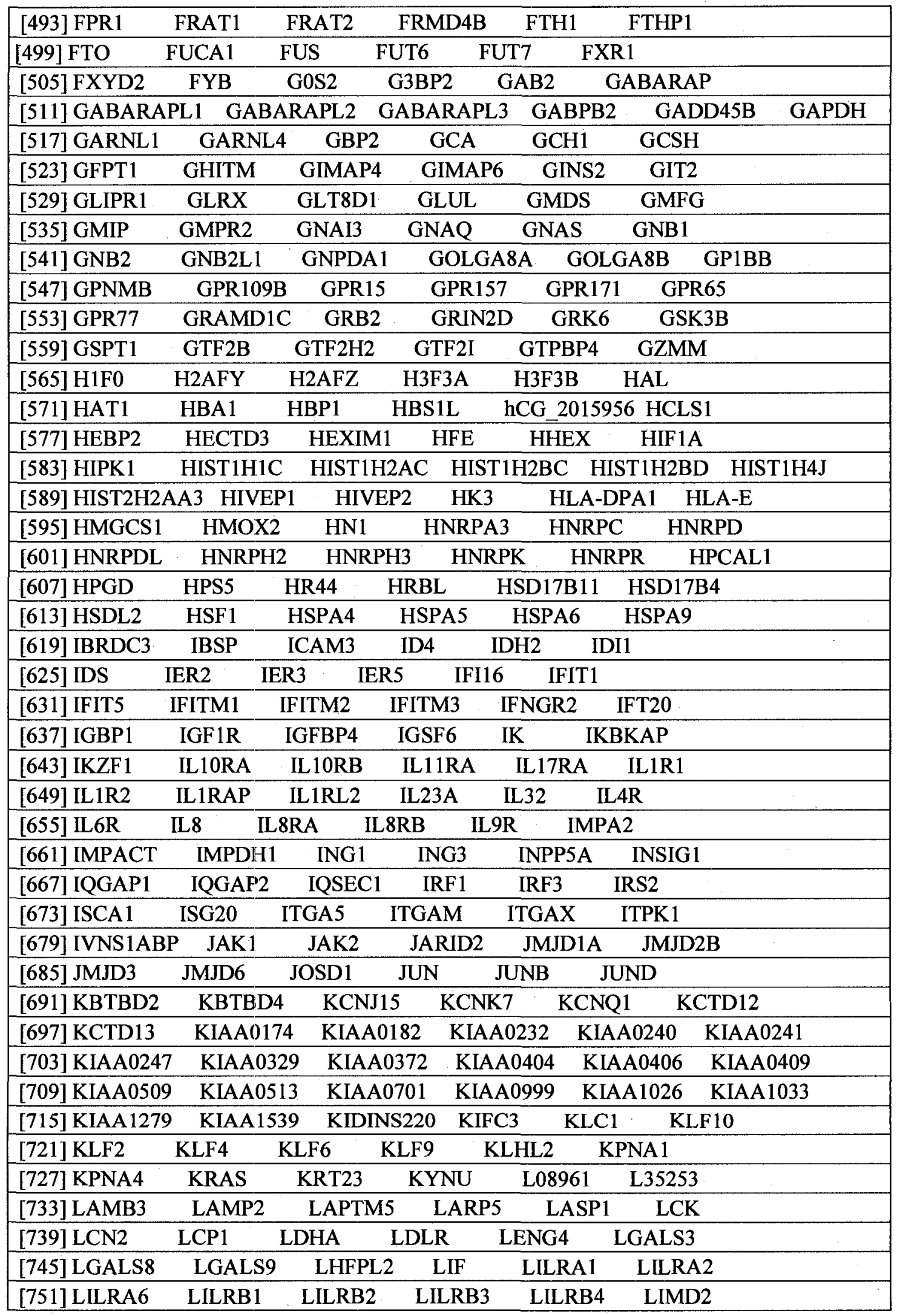




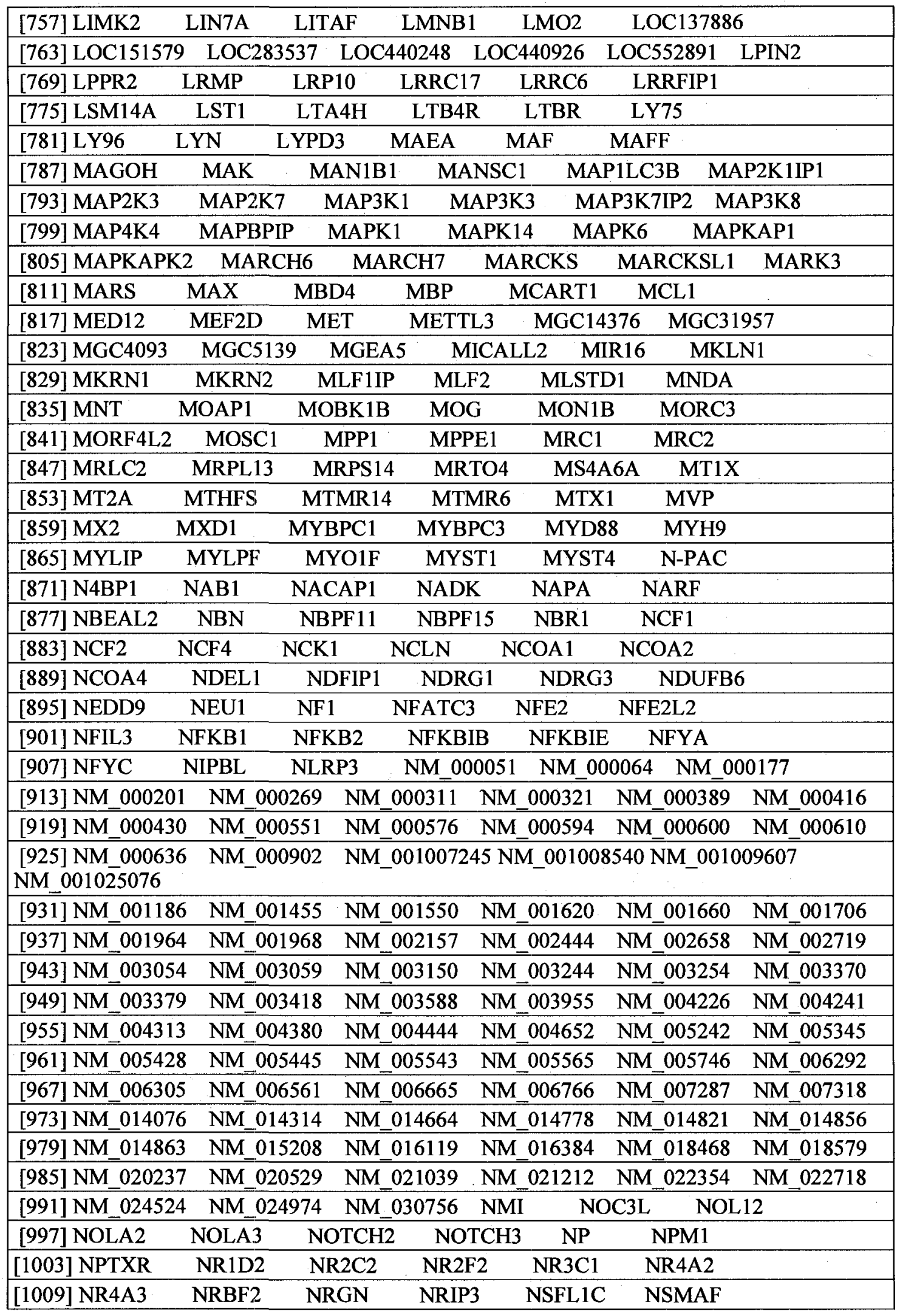




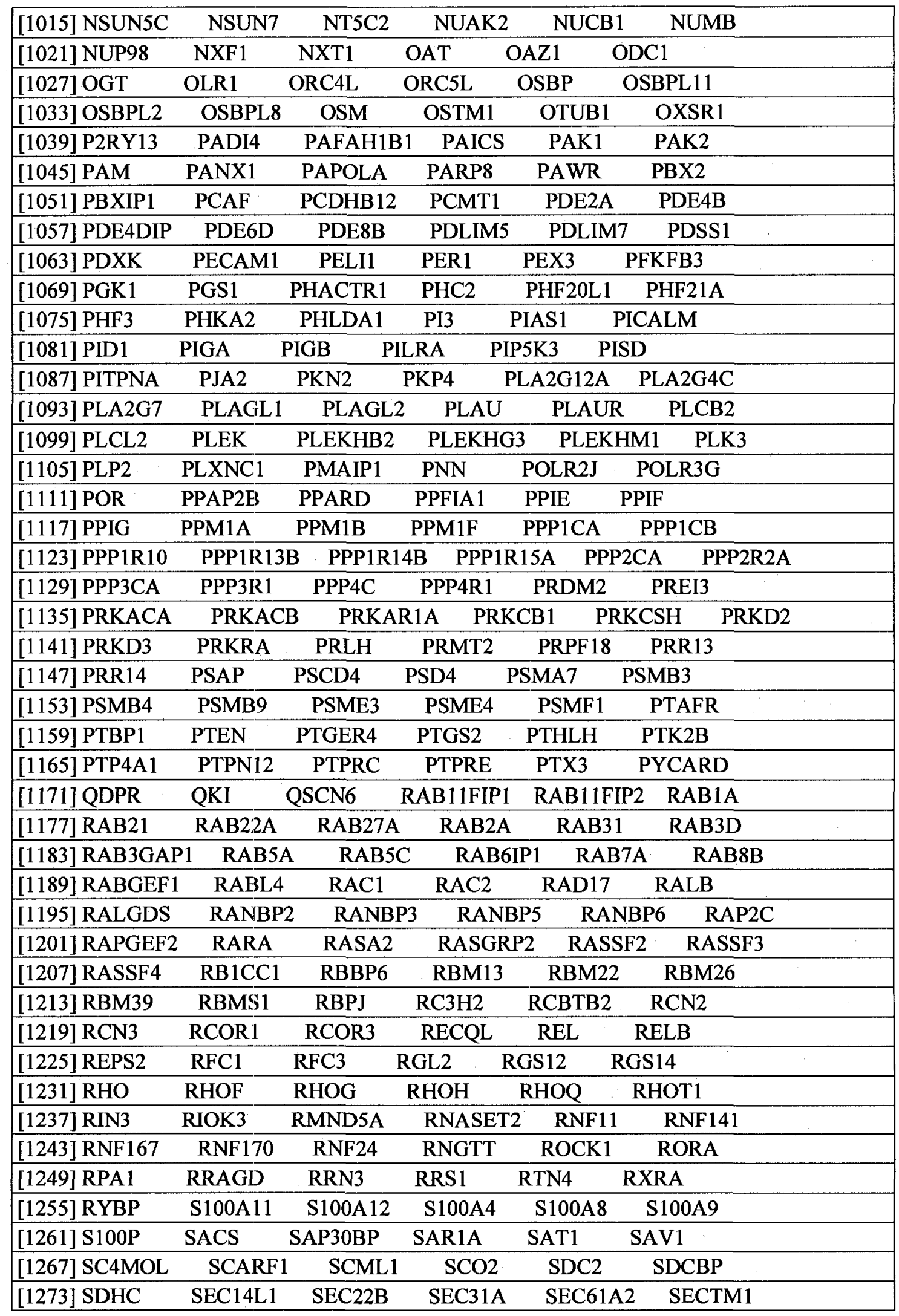




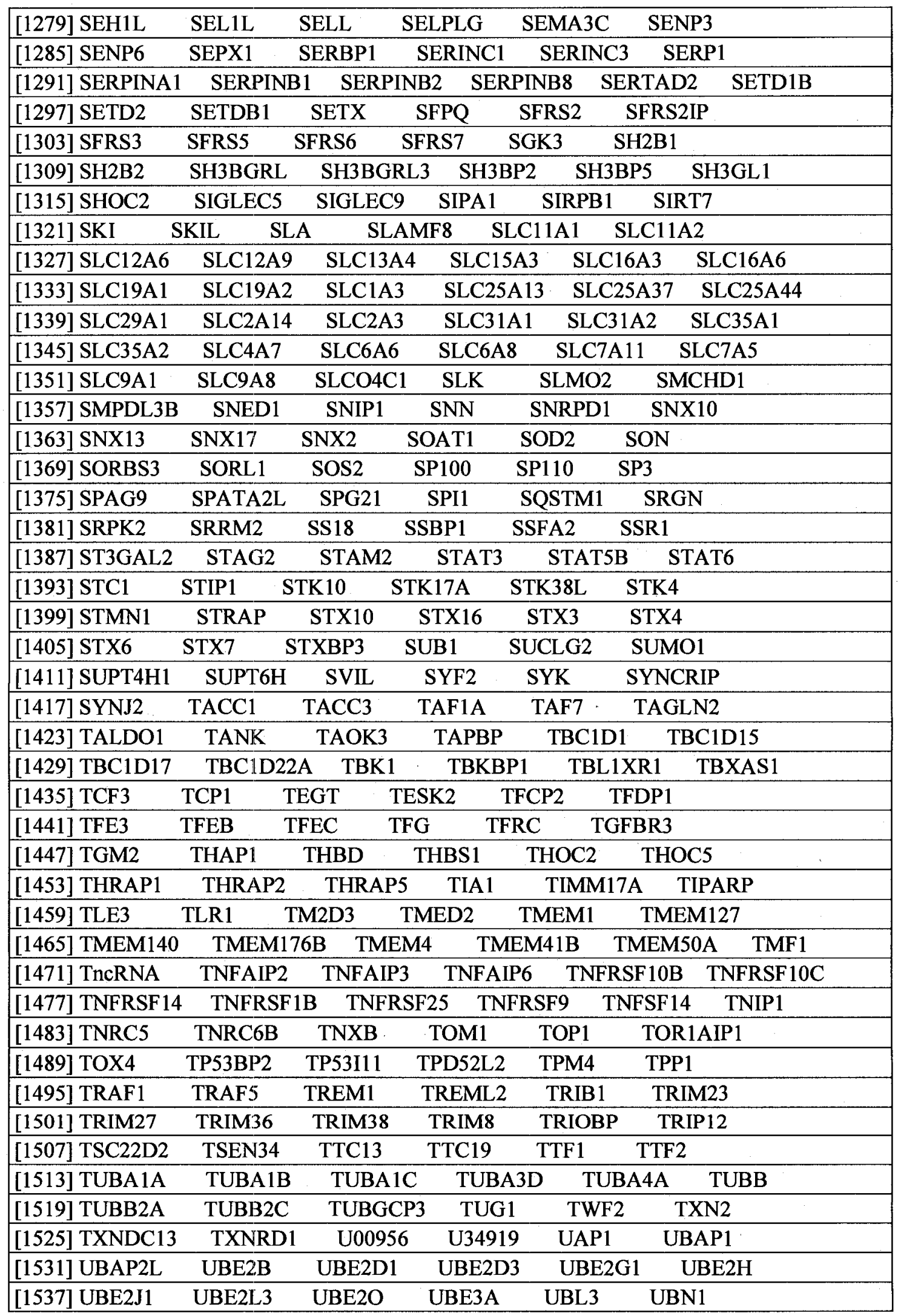




\begin{tabular}{|c|c|c|c|c|c|}
\hline [1543] UBR2 & UBTD1 & UBTF & UBXD2 & ULK1 & UNC119 \\
\hline [1549] UNC50 & UPF 1 & UPF3A & UQCRC2 & UROS & USH2A \\
\hline [1555] USP10 & USP15 & USP3 & USP32 & USP & USP36 \\
\hline [1561] USP8 & UTX & VAMP2 & VAMP3 & $\mathrm{VCL}$ & VCPIP1 \\
\hline [1567] VDR & VEGFA & VIL2 & VIM & S13C & VPS24 \\
\hline [1573] VPS26A & VPS37B & VRK1 & W88821 & WAC & WDR1 \\
\hline [1579] WDR26 & WDR47 & WDR8 & WIPF1 & WTAP & XBP1 \\
\hline$[1585]$ XM 09458 & \multicolumn{2}{|c|}{$81 \quad \mathrm{XM} \quad 370635$} & 374529 XM & $378250 \quad \mathrm{XP}$ & \multirow{2}{*}{$\frac{\text { XPO6 }}{\text { YKT6 }}$} \\
\hline [1591] XR_000228 & $8 \mathrm{XRCC} 4$ & XRCC5 & YIPF3 & YIPF4 & \\
\hline [1597] YPEL5 & YTHDC2 & YTHDF3 & YWHAB & YWHAE & YWHAZ \\
\hline [1603] ZBTB1 & ZBTB43 & ZC3H11A & $\mathrm{ZC} 3 \mathrm{H} 12 \mathrm{~A}$ & ZCCHC6 & ZDHHC18 \\
\hline [1609] ZEB1 & ZFAND3 & ZFAND6 & ZFP36 & ZFP36L2 & ZFX \\
\hline [1615] ZFYVE26 & $\mathrm{ZHX} 2$ & ZMIZ1 & ZMYM1 & ZMYM2 & ZNF12 \\
\hline [1621] ZNF148 & ZNF165 & ZNF180 & ZNF224 & ZNF227 & ZNF24 \\
\hline [1627] ZNF250 & ZNF267 & ZNF292 & ZNF350 & ZNF394 & ZNF467 \\
\hline [1633] ZNF508 & ZNF518 & ZNF573 & ZNF588 & ZNF668 & ZNF7 \\
\hline [1639] ZNF710 & ZNF750 & ZNF804A & ZUBR1 & $\mathrm{ZYX}$ & ZZEF1 \\
\hline
\end{tabular}


Table A.2 Response gene set for GDS1428 treatment group. Total 1902 response genes.

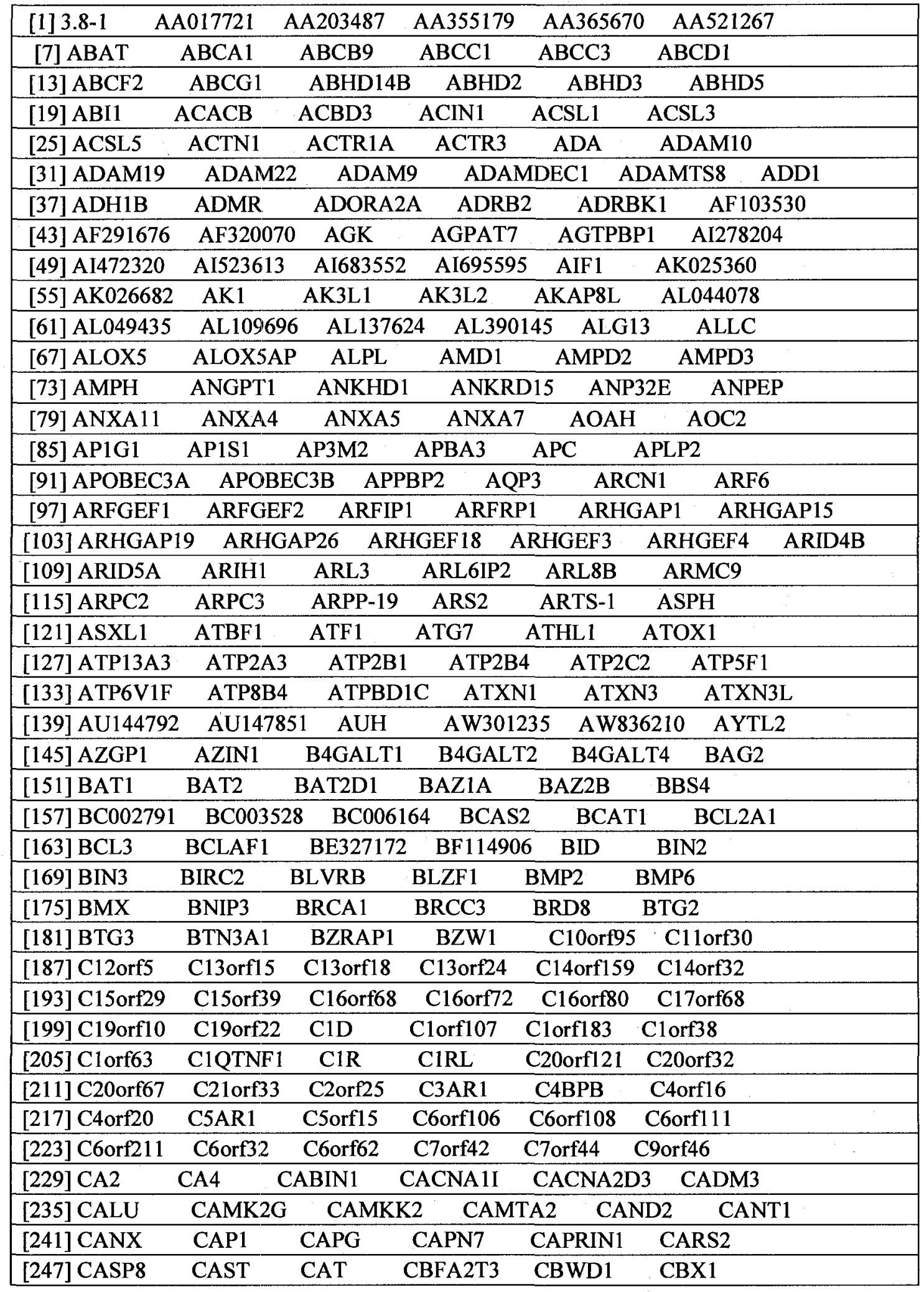




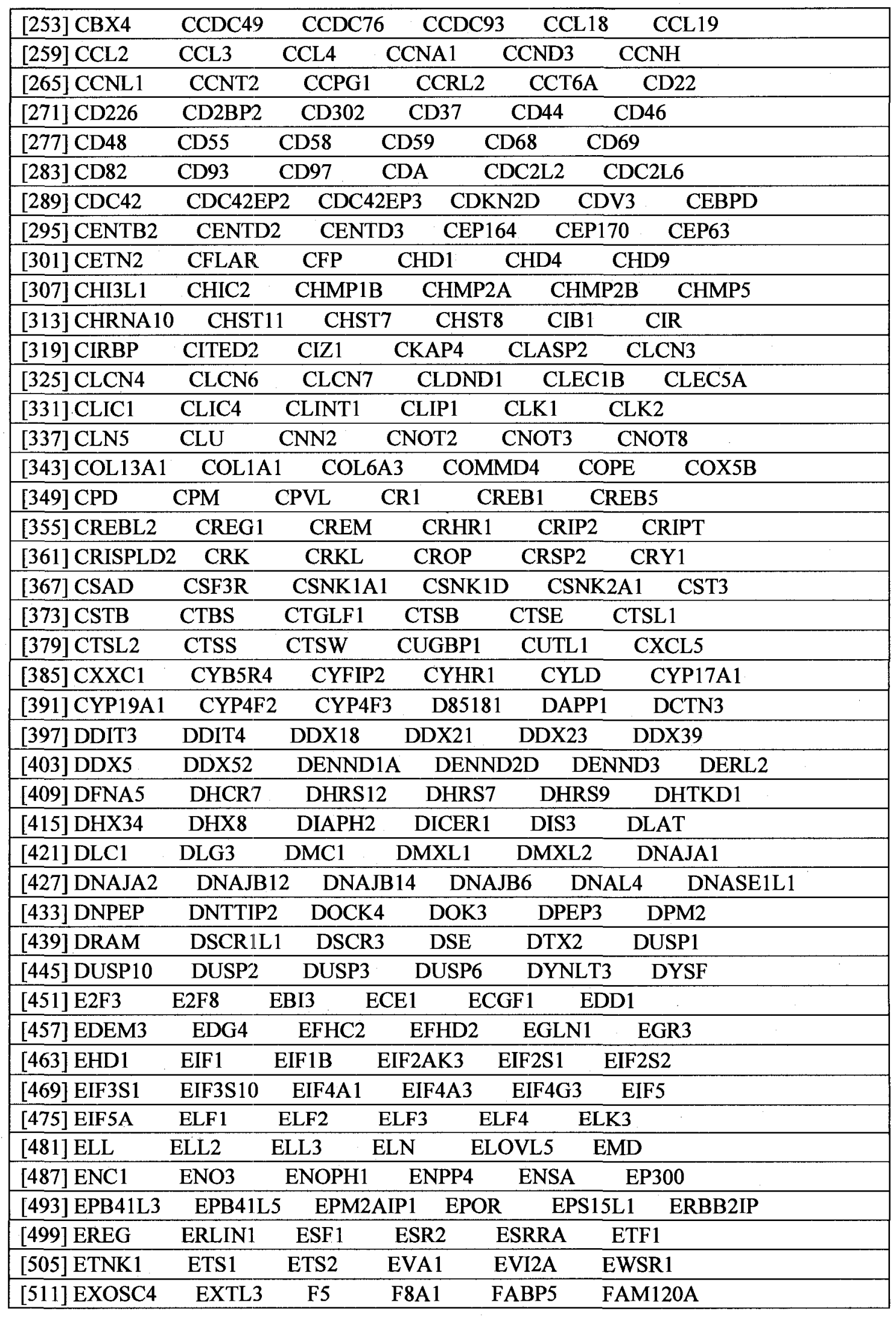




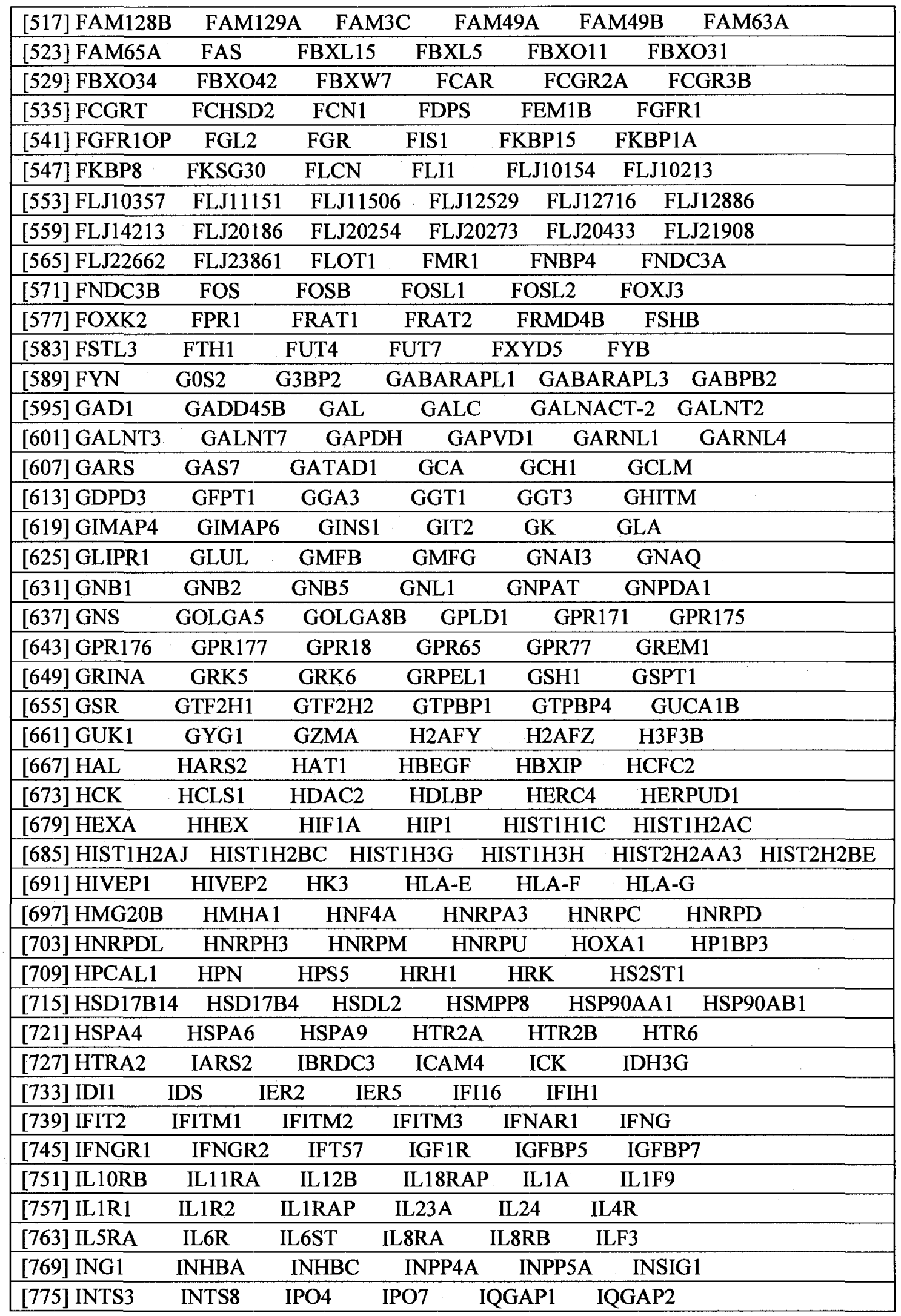




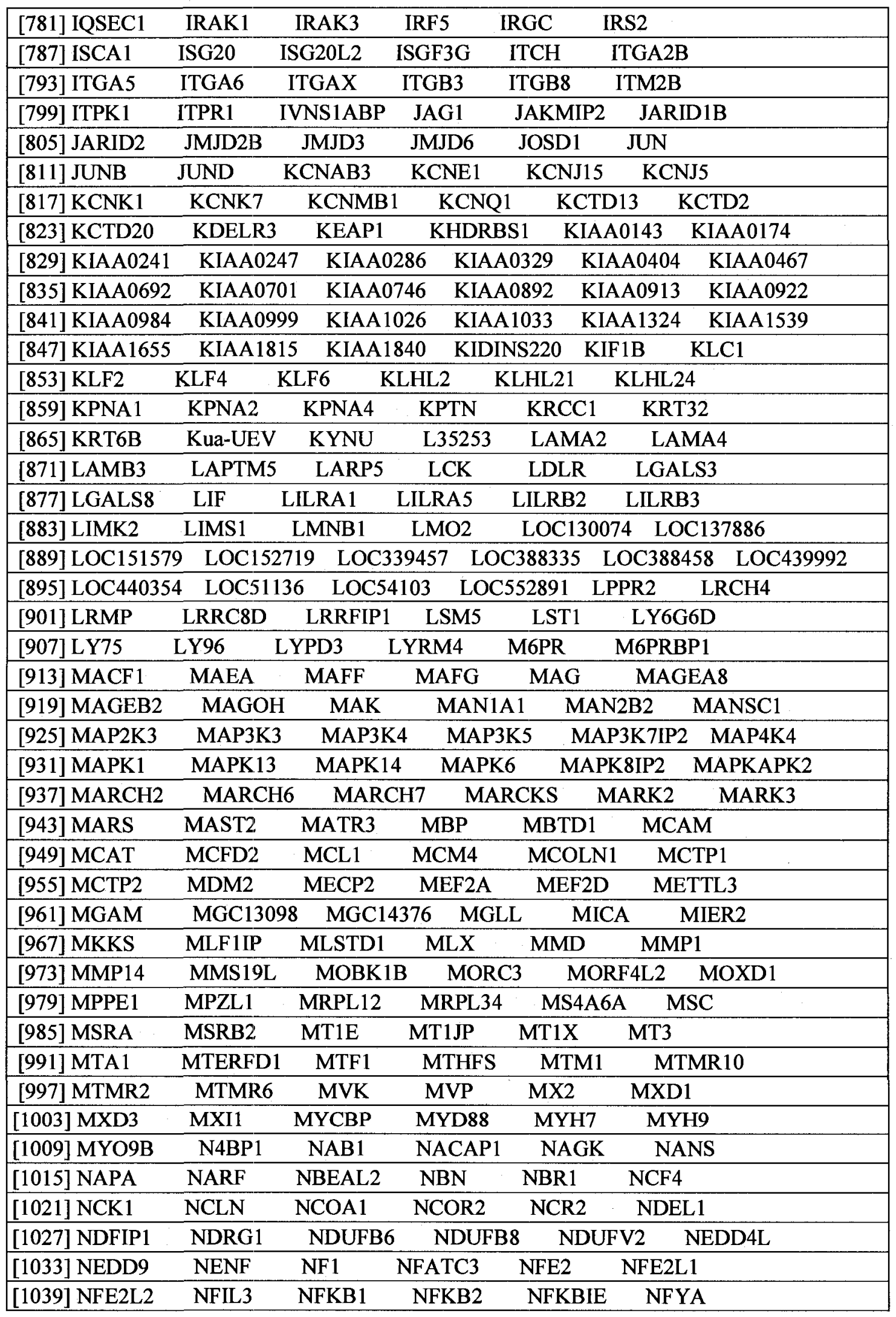




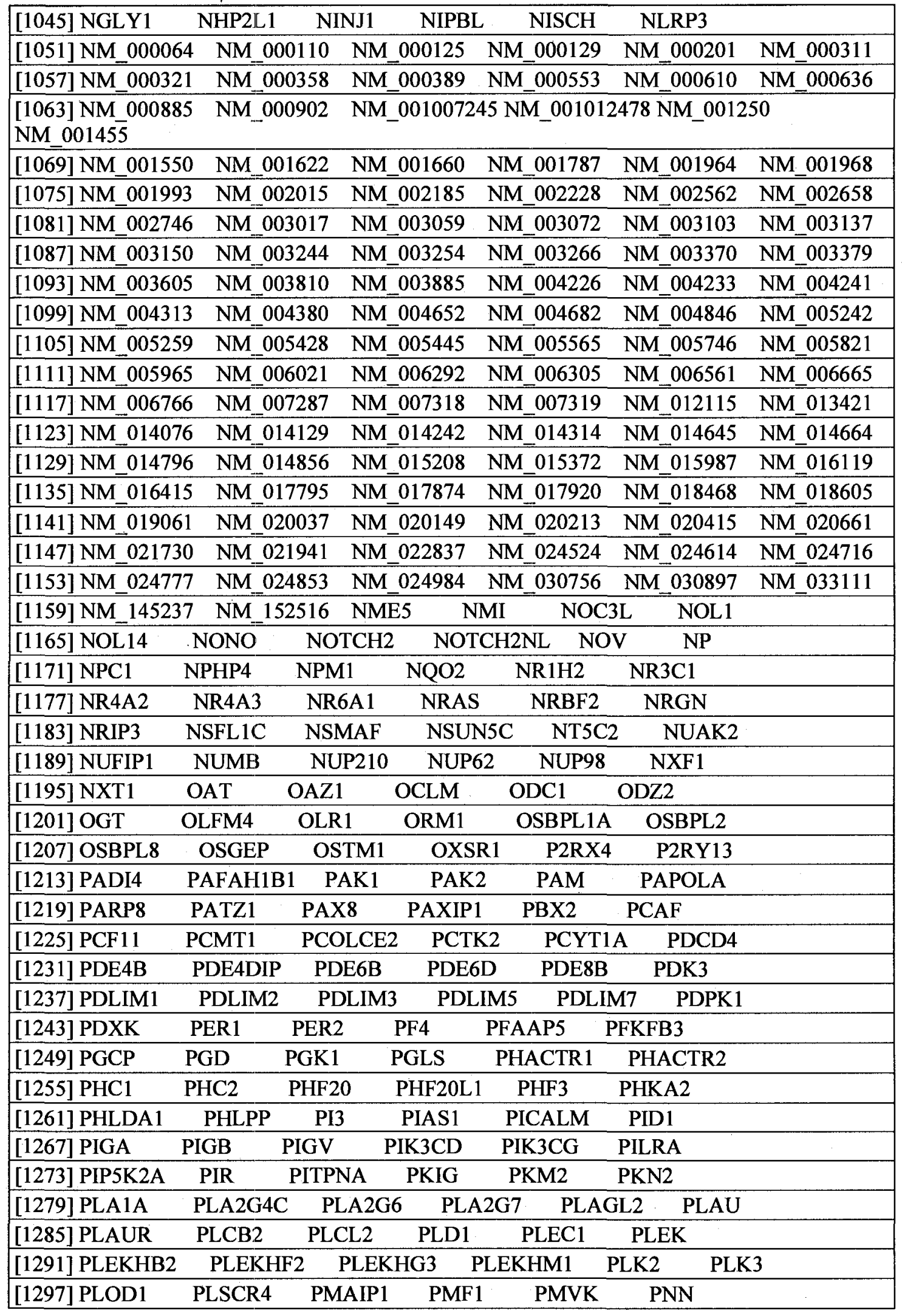




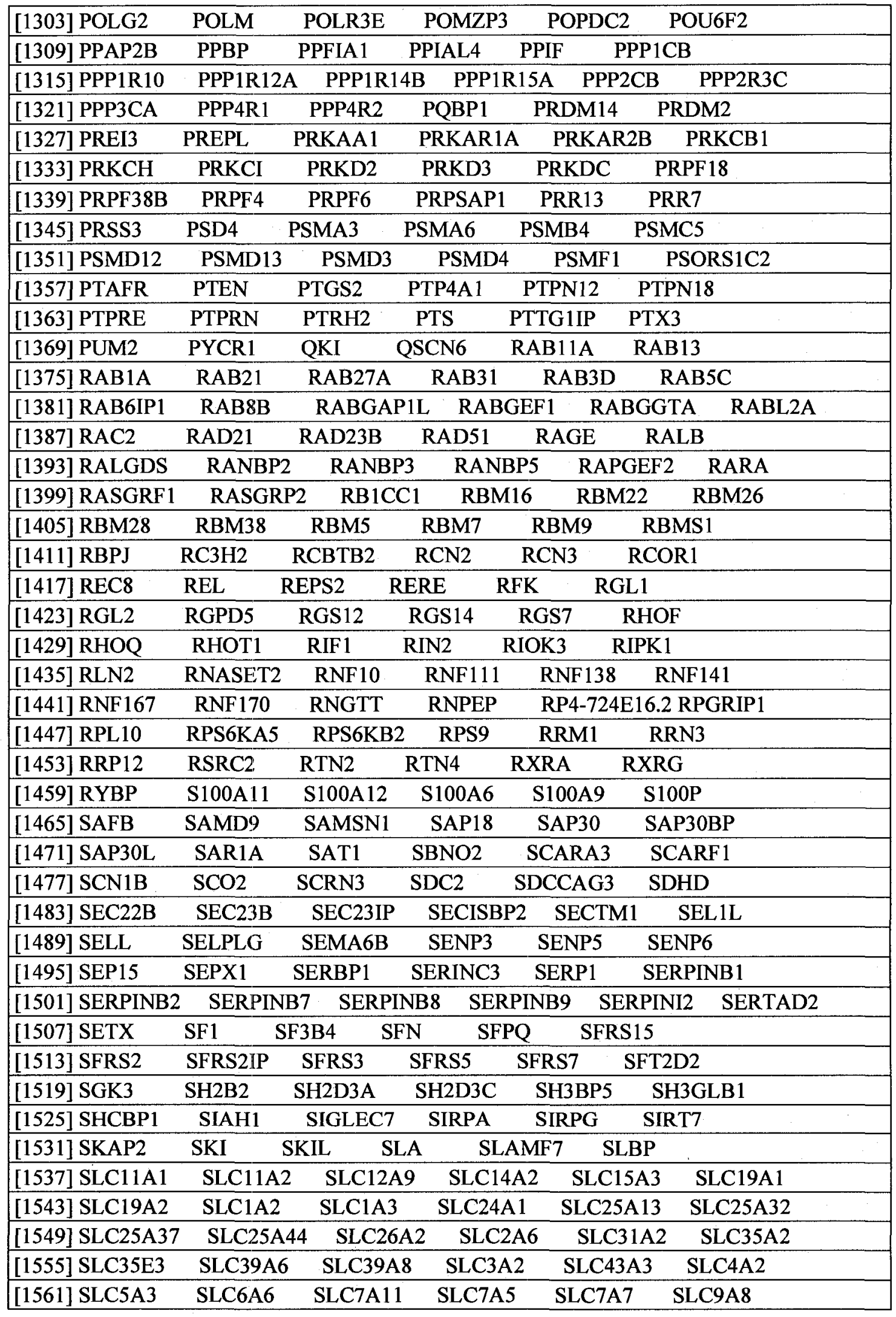




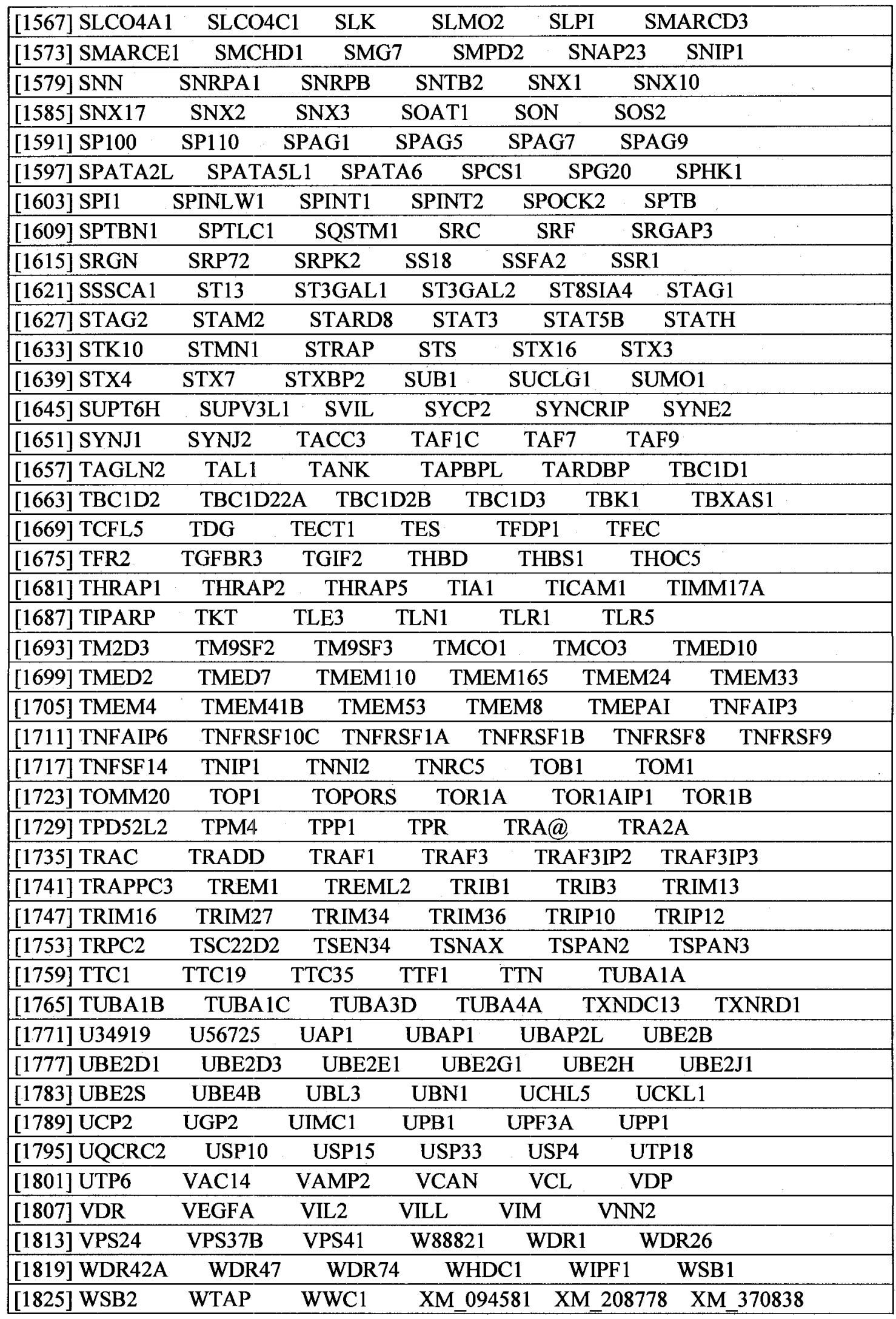




\begin{tabular}{|llllllllll|}
\hline$[1831]$ & XM_372632 & XM_374529 & XM_378250 & XM_496132 & XM_496217 & XM_497663 \\
\hline$[1837]$ & XM_498825 & XM_ & X98877 & XM_499165 & XPO1 & XPO6 & YAF2 \\
\hline$[1843]$ & YIPF4 & YIPF6 & YPEL5 & YRDC & YTHDC1 & YWHAZ \\
\hline$[1849]$ YY1 & ZBED1 & ZBTB1 & ZBTB17 & ZBTB3 & ZBTB43 \\
\hline$[1855]$ ZC3H12A & ZC3H7A & ZCCHC10 & ZCCHC14 & ZCCHC6 & ZDHHC17 \\
\hline$[1861]$ ZEB1 & ZEB2 & ZFAND5 & ZFP36 & ZFP36L2 & ZFX \\
\hline$[1867]$ ZFYVE26 & ZH2C2 & ZHX2 & ZMAT3 & ZMYM1 & ZMYND10 \\
\hline$[1873]$ ZNF124 & ZNF155 & ZNF165 & ZNF177 & ZNF202 & ZNF221 \\
\hline$[1879]$ & ZNF225 & ZNF238 & ZNF24 & ZNF250 & ZNF254 & ZNF259 \\
\hline$[1885]$ ZNF267 & ZNF277P & ZNF331 & ZNF350 & ZNF394 & ZNF500 \\
\hline$[1891]$ ZNF508 & ZNF552 & ZNF586 & ZNF589 & ZNF652 & ZNF668 \\
\hline$[1897]$ ZNF675 & ZNF692 & ZNF783 & ZNF84 & ZYX & ZZEF1 \\
\hline
\end{tabular}


Table A.3 The common response gene set for GDS1428 treatment group and control group. Total 894 genes.

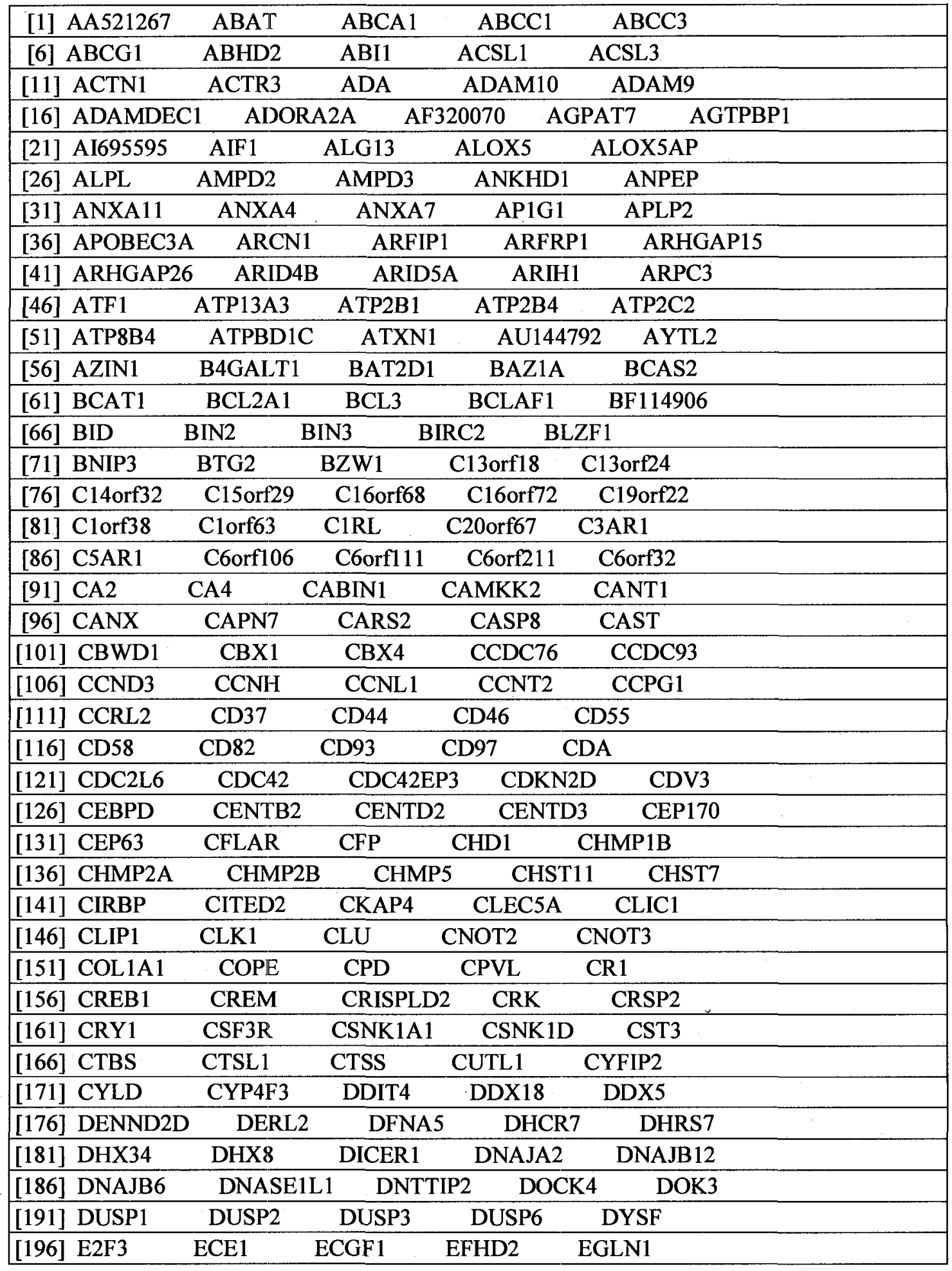




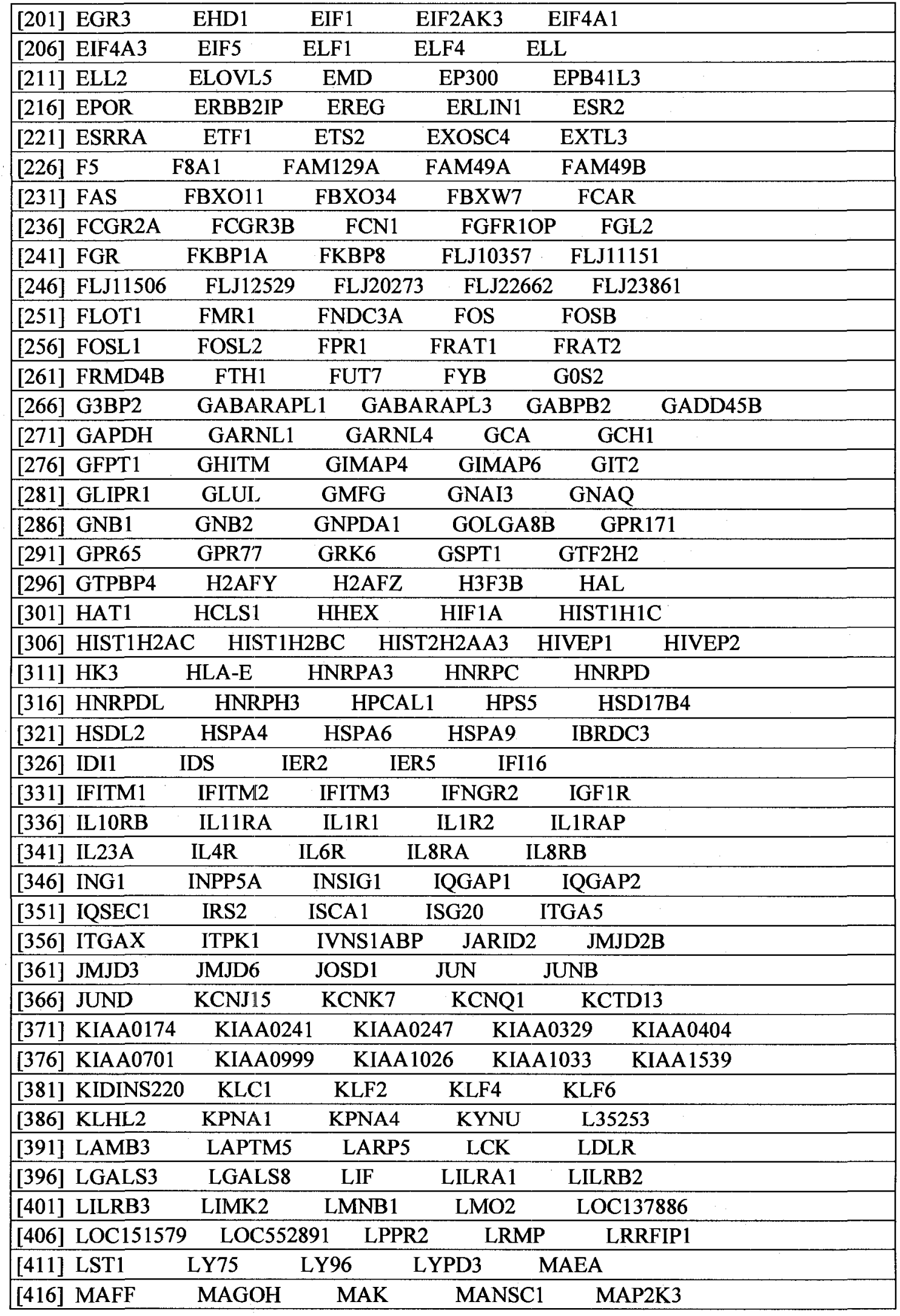




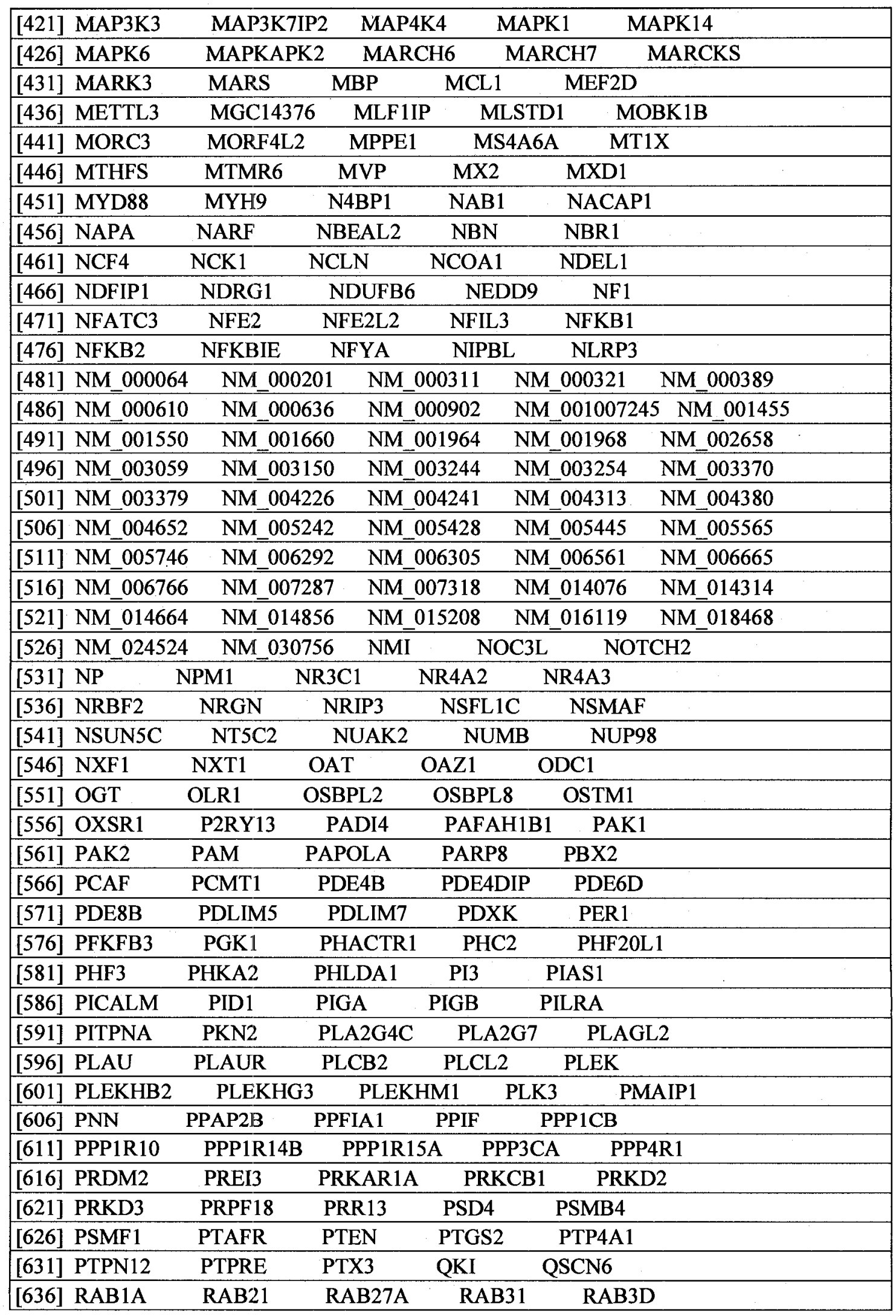




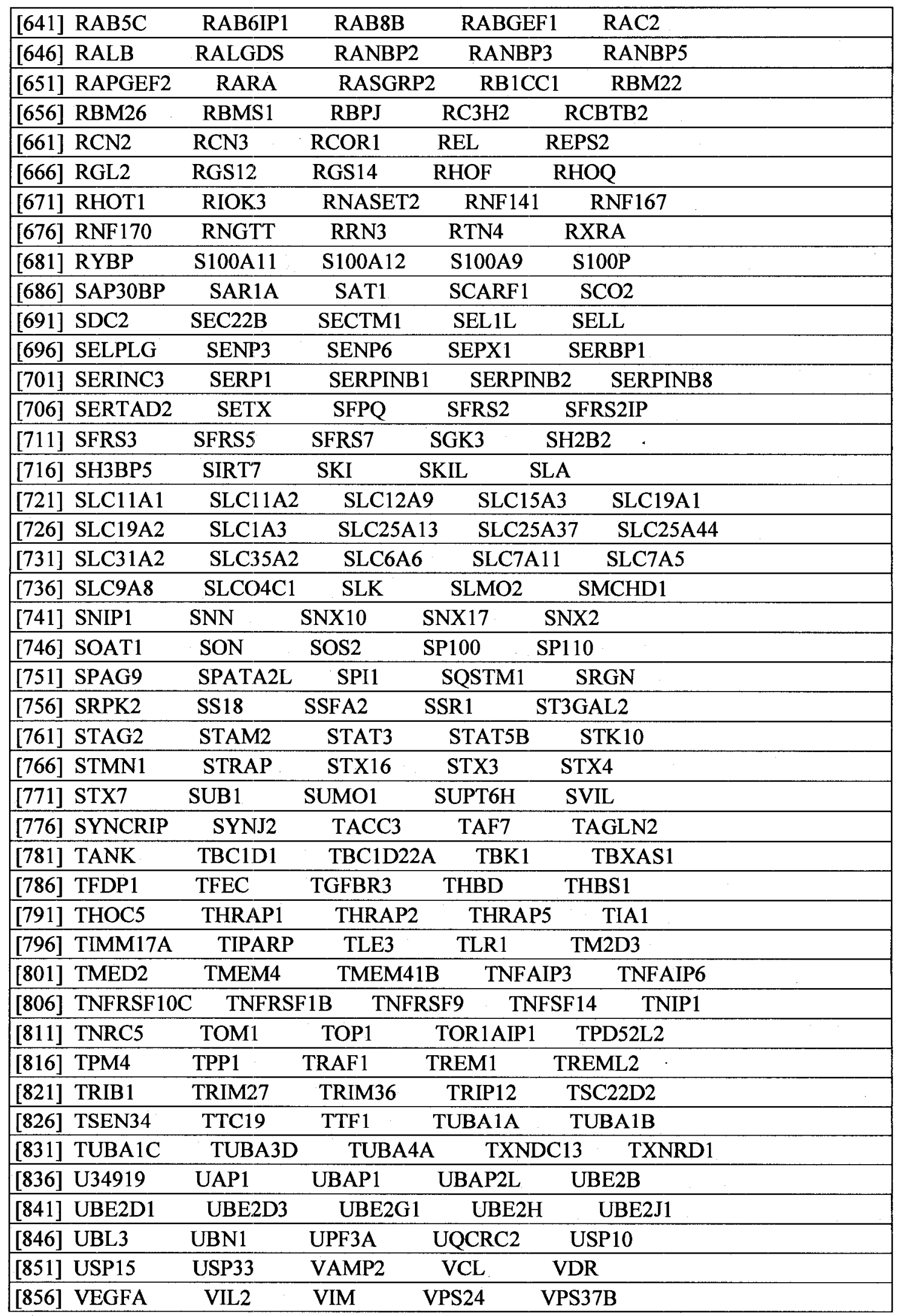




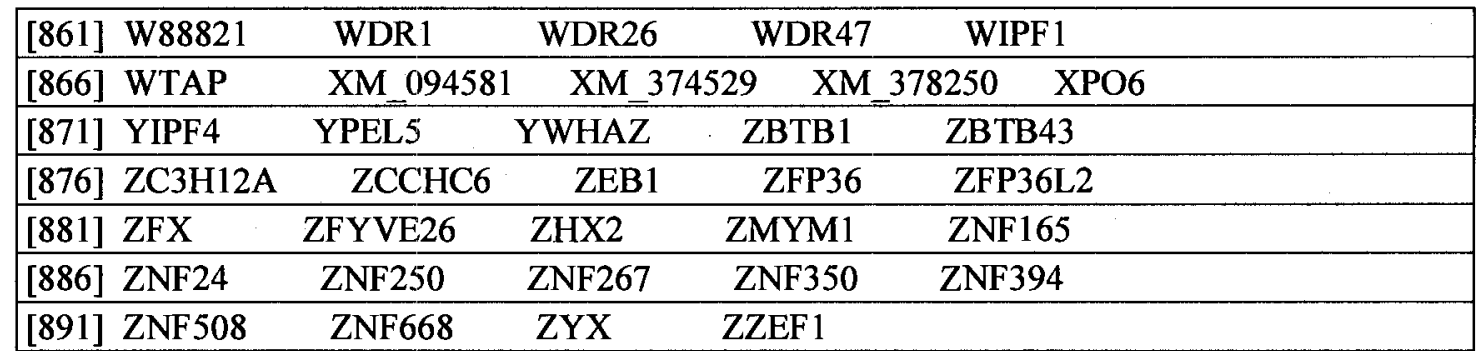


Table A.4. The response gene set only for GDS1428 control group. Total 750 genes.

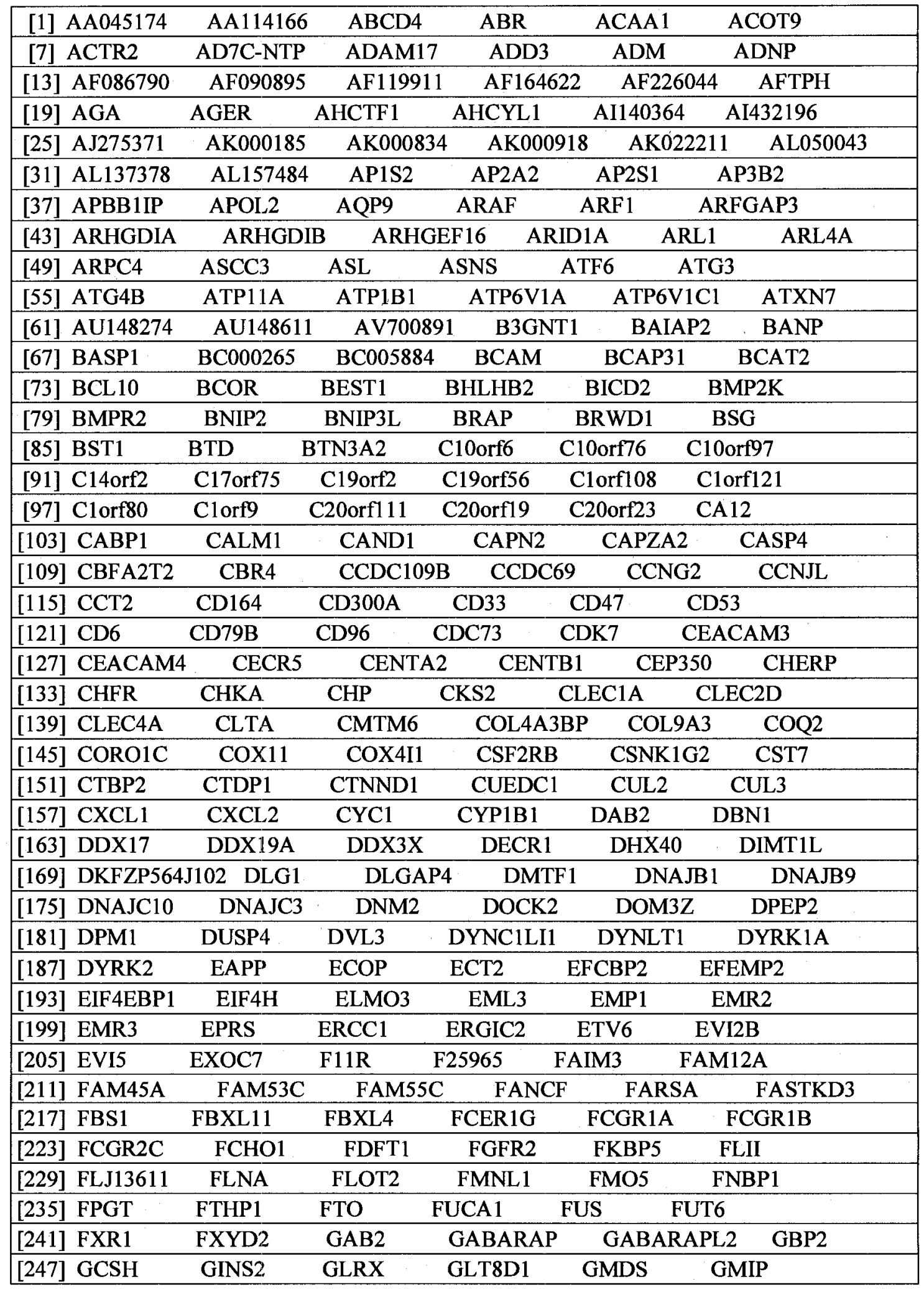




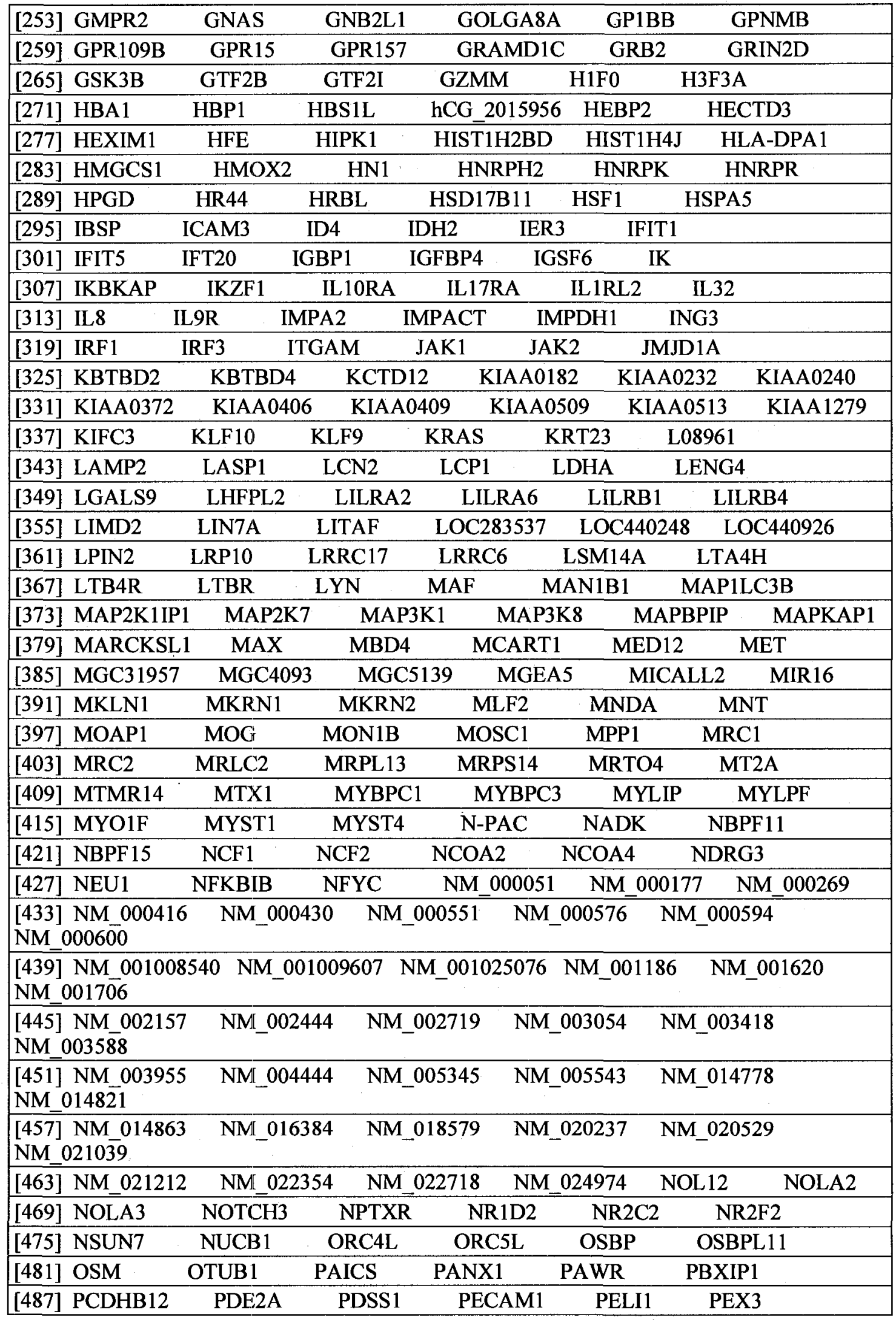




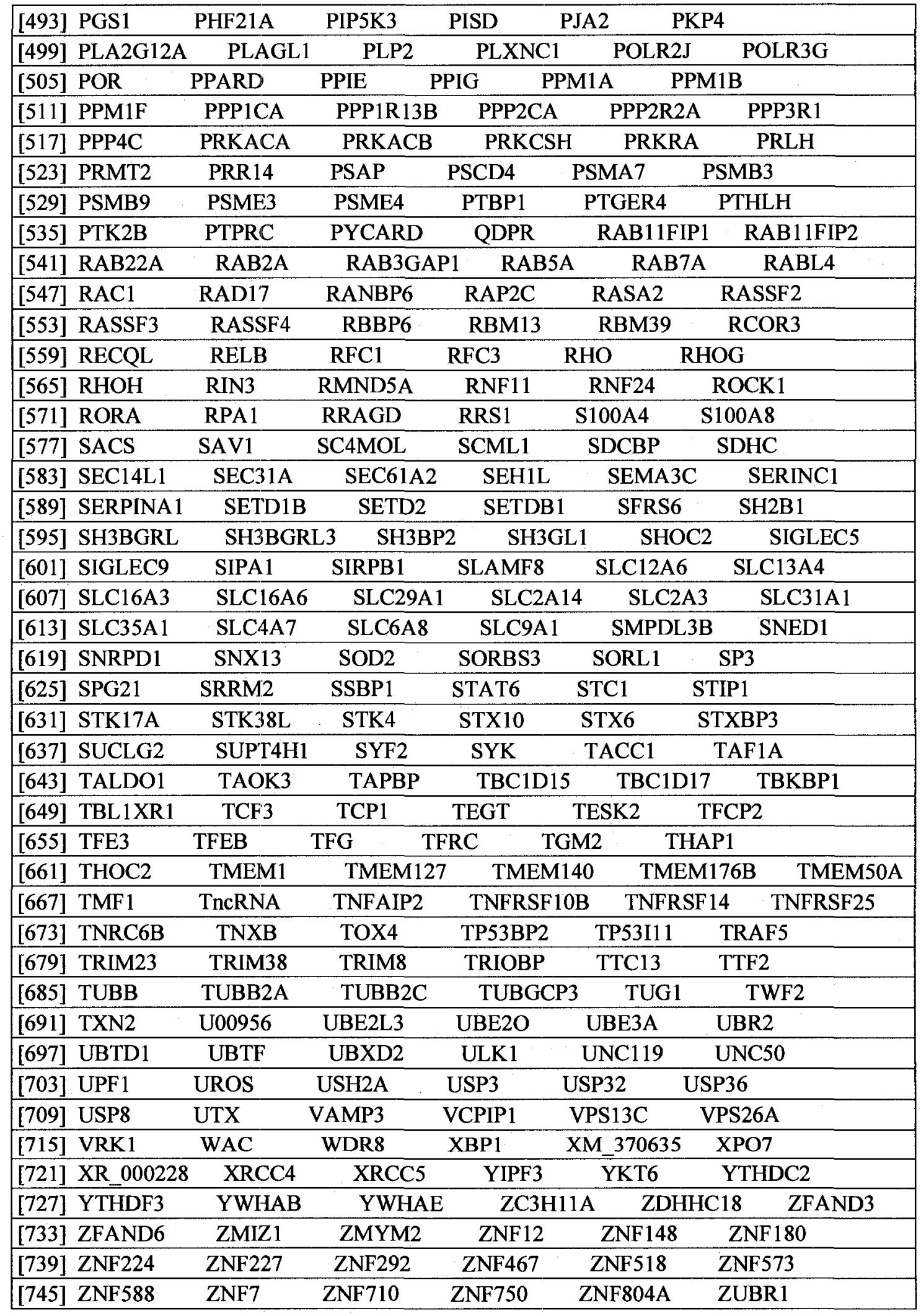


Table A.5. The response gene set only for GDS1428 treatment group. Total 1008 genes.

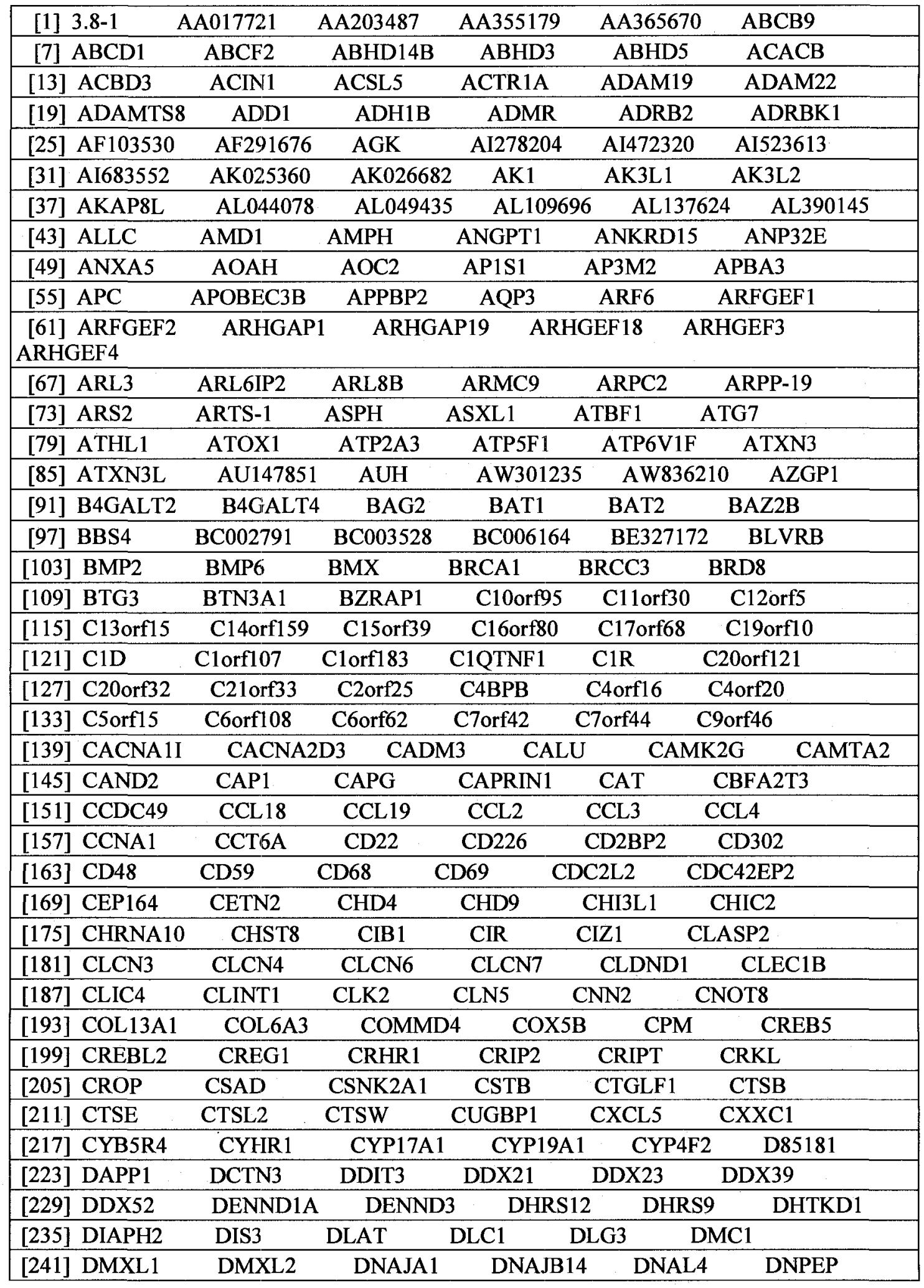




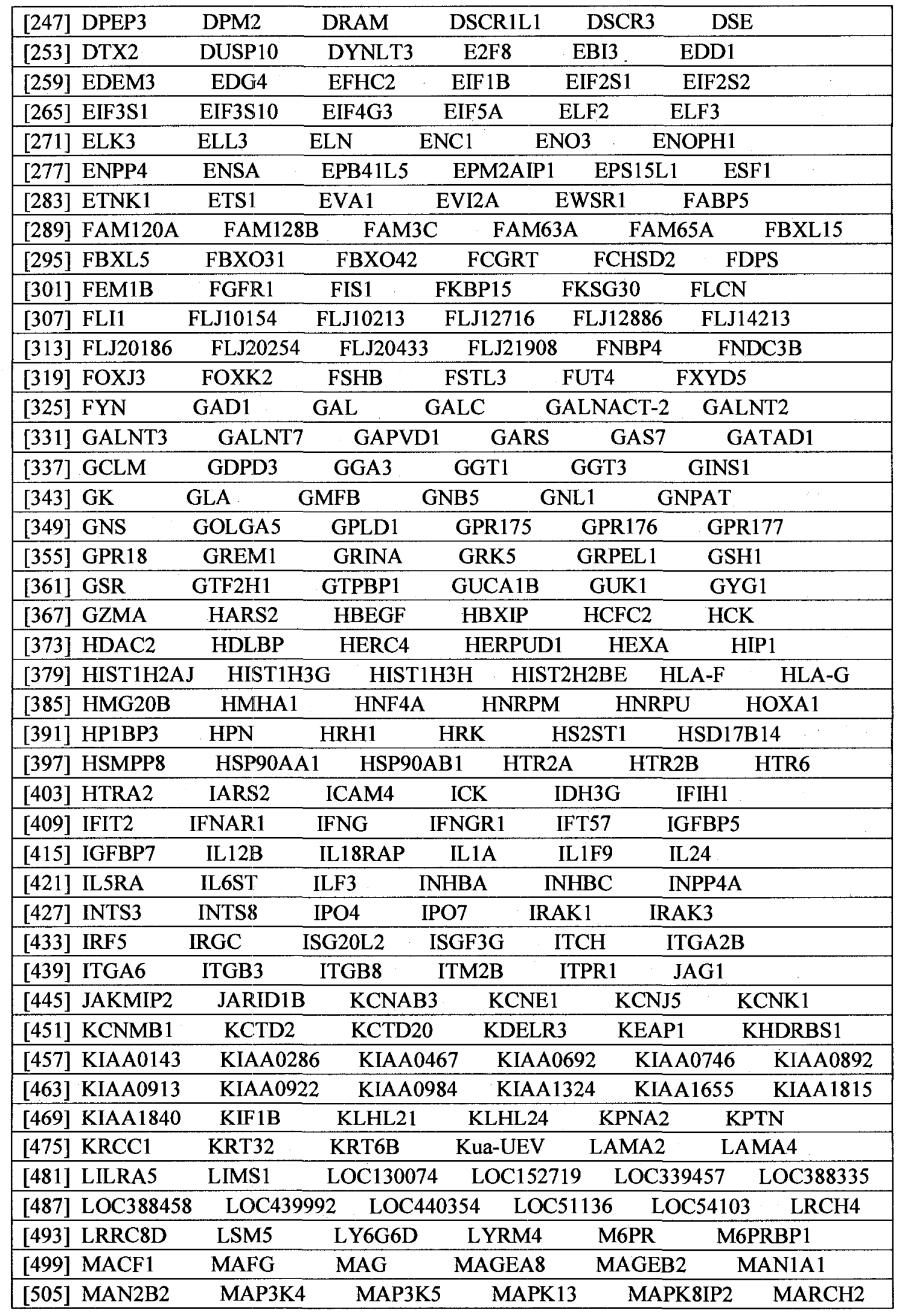




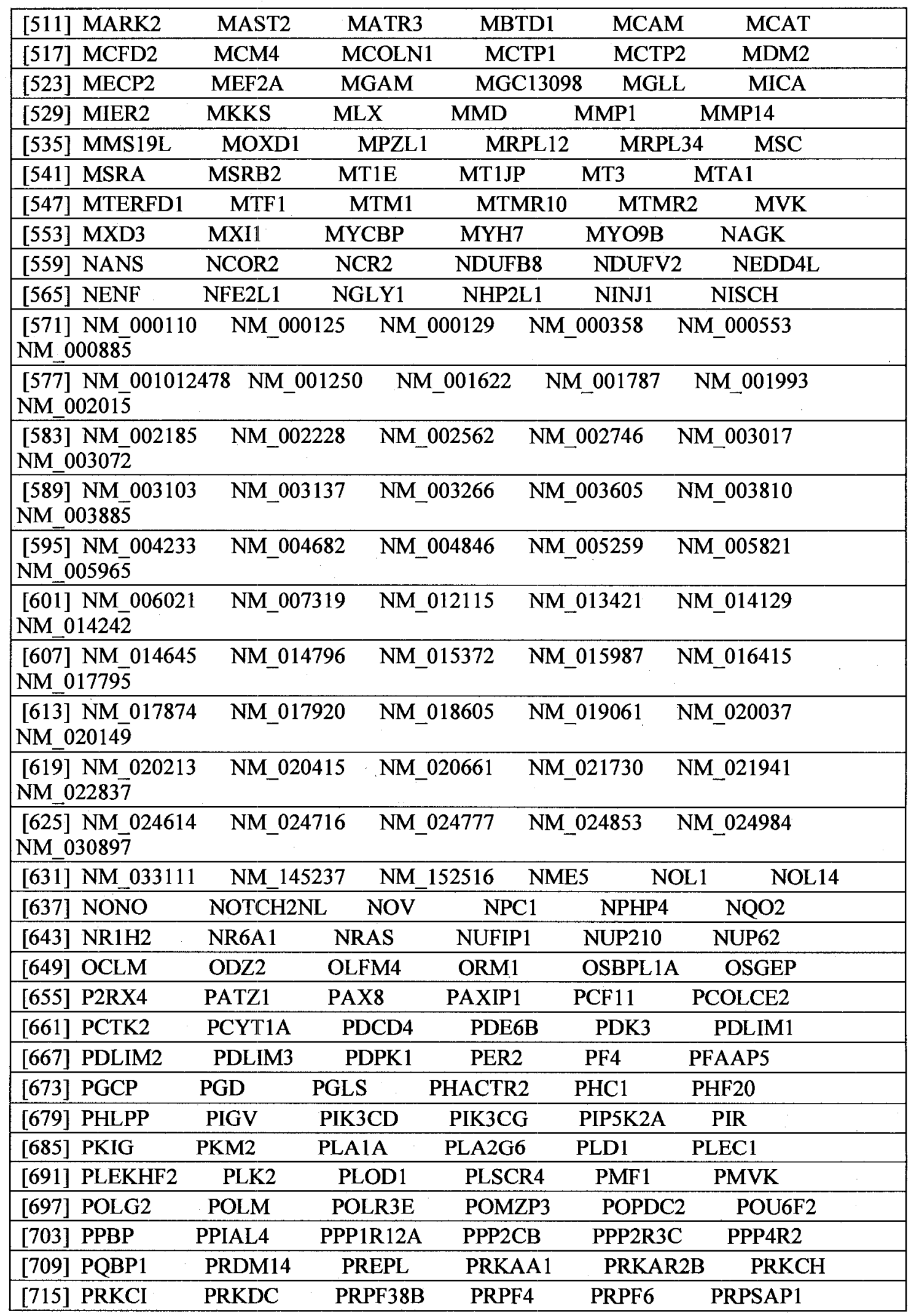




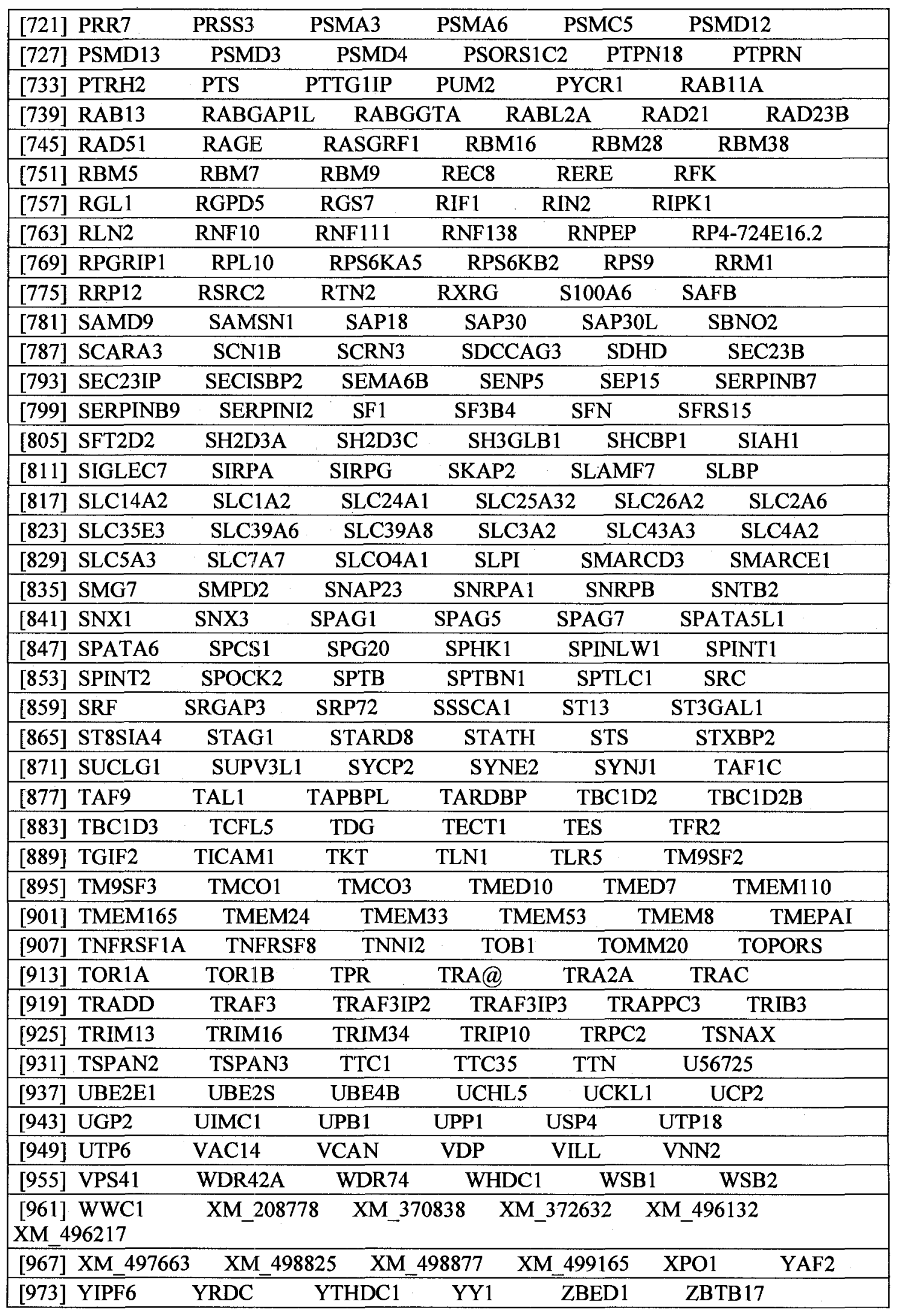




\begin{tabular}{|llccccc|}
\hline [979] & ZBTB3 & ZC3H7A & ZCCHC10 & ZCCHC14 & ZDHHC17 & ZEB2 \\
\hline [985] & ZFAND5 & ZH2C2 & ZMAT3 & ZMYND10 & ZNF124 & ZNF155 \\
\hline [991] & ZNF177 & ZNF202 & ZNF221 & ZNF225 & ZNF238 & ZNF254 \\
\hline [997] & ZNF259 & ZNF277P & ZNF331 & ZNF500 & ZNF552 & ZNF586 \\
\hline [1003] & ZNF589 & ZNF652 & ZNF675 & ZNF692 & ZNF783 & ZNF84 \\
\hline
\end{tabular}


Table A.6. The common set of differentially regulated gene set (gene set A) and the response gene set under treatment (gene set $\mathrm{B}$ ). Total 232 genes.

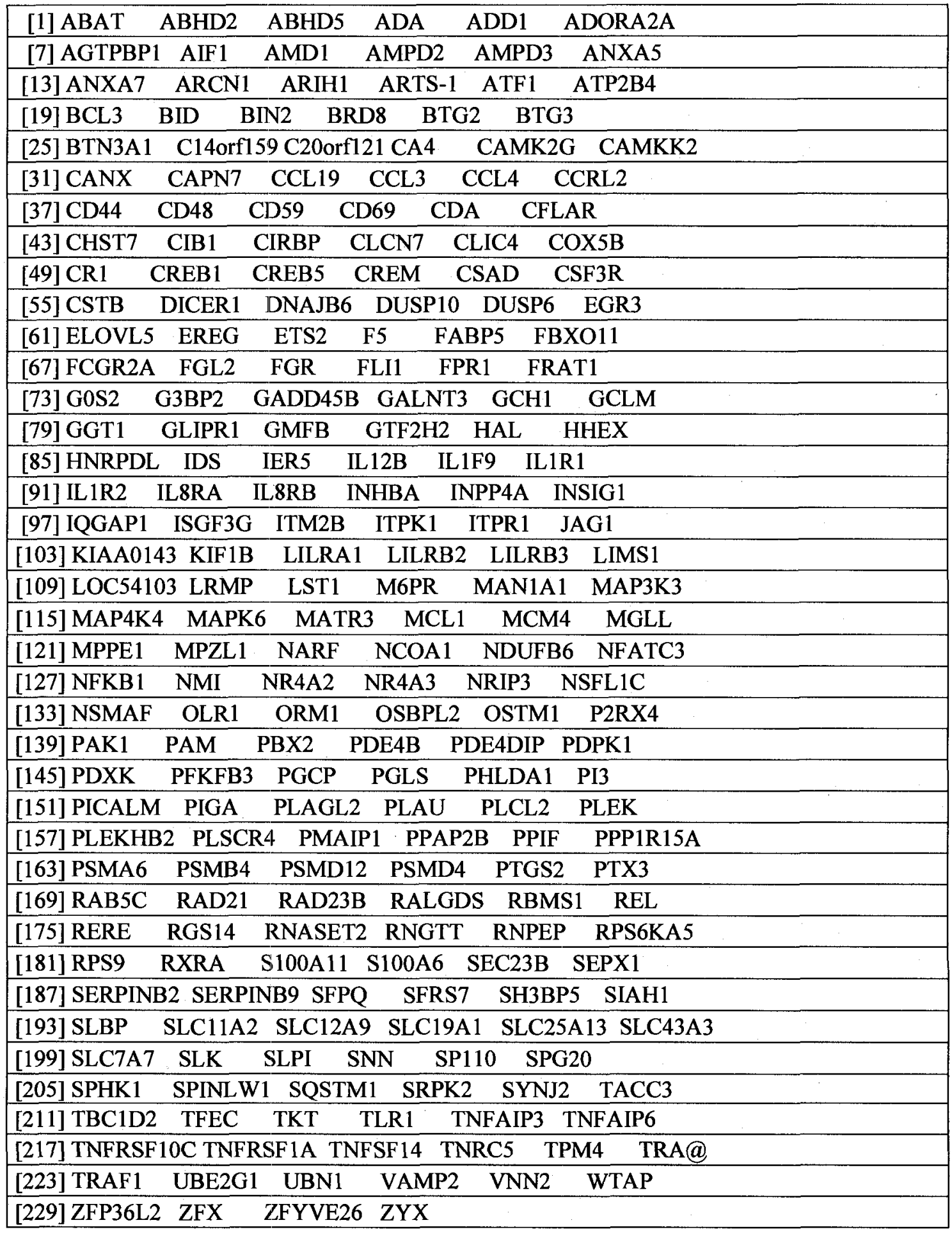


Table A.7. Those genes only in differentially regulated gene set (gene set A) but not in the response gene set under treatment (gene set $B$ ). Total 578 genes.

\begin{tabular}{|c|c|c|c|c|}
\hline [1] $101 \mathrm{~F} 6$ & 2TRPA1 & ABCA7 & ACAT2 & ACINUS \\
\hline [6] ACTN4 & ADAM17 & ADAM8 & ADCY3 & ADD3 \\
\hline [11] ADRA1A & ADSL & AHCY & $\mathrm{AK} 2$ & AK3 \\
\hline [16] AKT1 & ALAS1 & ANP32B & APG4B & AQP9 \\
\hline [21] ARFGAP3 & ARHGAI & P11A ARHC & IGAP4 & ARHGEF6 \\
\hline [26] ARHQ & ARHT1 & ARPC4 & ASK & ATF5 \\
\hline [31] ATP1B1 & ATP5J & ATP6V1A1 & ATPIF1 & AUP1 \\
\hline [36] B3GNT4 & B4GALT5 & BAGE & BCAP31 & BCL10 \\
\hline [41] BCL11A & BCOR & BFAR & BICD2 & BIGM103 \\
\hline [46] BM045 & BMP2K & BRAF & BRD1 & BTBD14A \\
\hline [51] BTF & BTG1 & BTK & TN2A1 & TN3A2 \\
\hline [56] BTN3A3 & C11orf10 & C13orf10 & C14orf109 & C14orf147 \\
\hline [61] C19orf7 & C1orf16 & C1orf 24 & C1QBP & C20orf104 \\
\hline [66] C21 orf66 & C21 orf91 & C22orf19 & $\mathrm{C} 3$ & 9 orf10 \\
\hline [71] Cab45 & CABC1 & CASC3 & CASP2 & CASP4 \\
\hline [76] CBX7 & CCL20 & CCNG2 & CCR1 & CCR3 \\
\hline [81] CCR9 & CD74 & CD9 & DC16 & $\mathrm{C} 34$ \\
\hline [86] CDC5L & CDC6 & CECR5 & CES1 & CGI-72 \\
\hline [91] CHES1 & ChGn & CHS1 & CHST2 & CHST6 \\
\hline [96] cig5 & CKLF & CLC & ECSF12 & LECSF6 \\
\hline [101] CLECSF9 & CLN2 & CMAH & COL15A1 & COL18A1 \\
\hline [106] COPEB & COPS3 & CORO1A & CPR8 & CRSP3 \\
\hline [111] CSNK1G2 & CSPG2 & CTBP1 & CTBP2 & CTNNA1 \\
\hline [116] CUGBP2 & CXCL 1 & CXCL2 & CXCL3 & DAMS \\
\hline [121] DARS & DCL-1 & DDEF1 & DGCR2 & DIAPH1 \\
\hline \multicolumn{5}{|c|}{$\begin{array}{l}\text { [126] DKFZP434C171 DKFZP566A1524 DKFZp586I1420 DKFZP586L151 } \\
\text { DKFZP586M1523 }\end{array}$} \\
\hline \multicolumn{2}{|c|}{ [131] DKFZp761P1010 DLEU } & DLGA & $\mathrm{DMN}$ & DNAH7 \\
\hline [136] DNAH9 & DNAJB99 & DNAJC8 & DOK1 & DPEP2 \\
\hline [141] DSIPI & E1B-AP5 & EEF1A1 & EGLN2 & EHD4 \\
\hline [146] EIF3S6IP & EIF4E & EIF4EL3 & EMR3 & ERAL1 \\
\hline [151] ETFA & EZH1 & EZI & 2RL1 & CL3 \\
\hline [156] FACL6 & FAD104 & FBS1 & FBXO9 & FCER1G \\
\hline [161] FCGR3A. & FDX1 & FETUB & FGF7 & FHOD1 \\
\hline [166] FLII & FLJ10055 & FLJ10707 & FLJ10726 & FLJ10858 \\
\hline [171] FLJ10996 & FLJ11036 & $6 \quad$ FLJ11088 & FLJ11142 & FLJ11259 \\
\hline [176] FLJ12150 & FLJ13॥95 & FLJ13386 & FLJ20038 & FLJ20189 \\
\hline [181] FLJ20274 & FLJ20287 & FLJ20373 & FLJ20449 & FLJ20502 \\
\hline [186] FLJ20530 & FLJ20559 & FLJ20811 & FLJ20986 & FLJ20989 \\
\hline [191] FLJ21047 & FLJ21308 & FLJ21588 & FLJ22169 & FLJ22649 \\
\hline [196] FLJ22843 & FLJ22938 & FLJ23056 & FLJ23142 & FLJ23231 \\
\hline
\end{tabular}




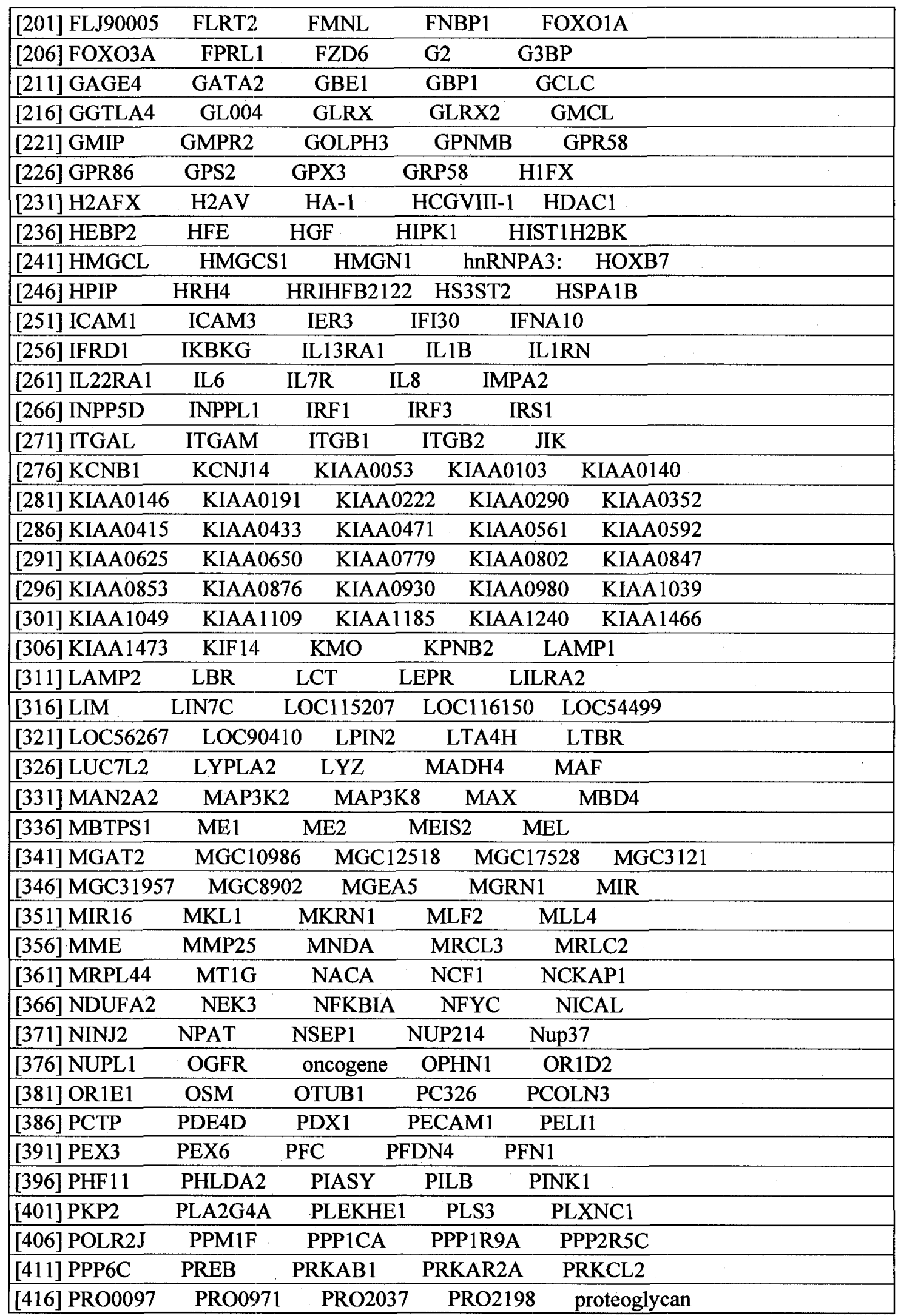




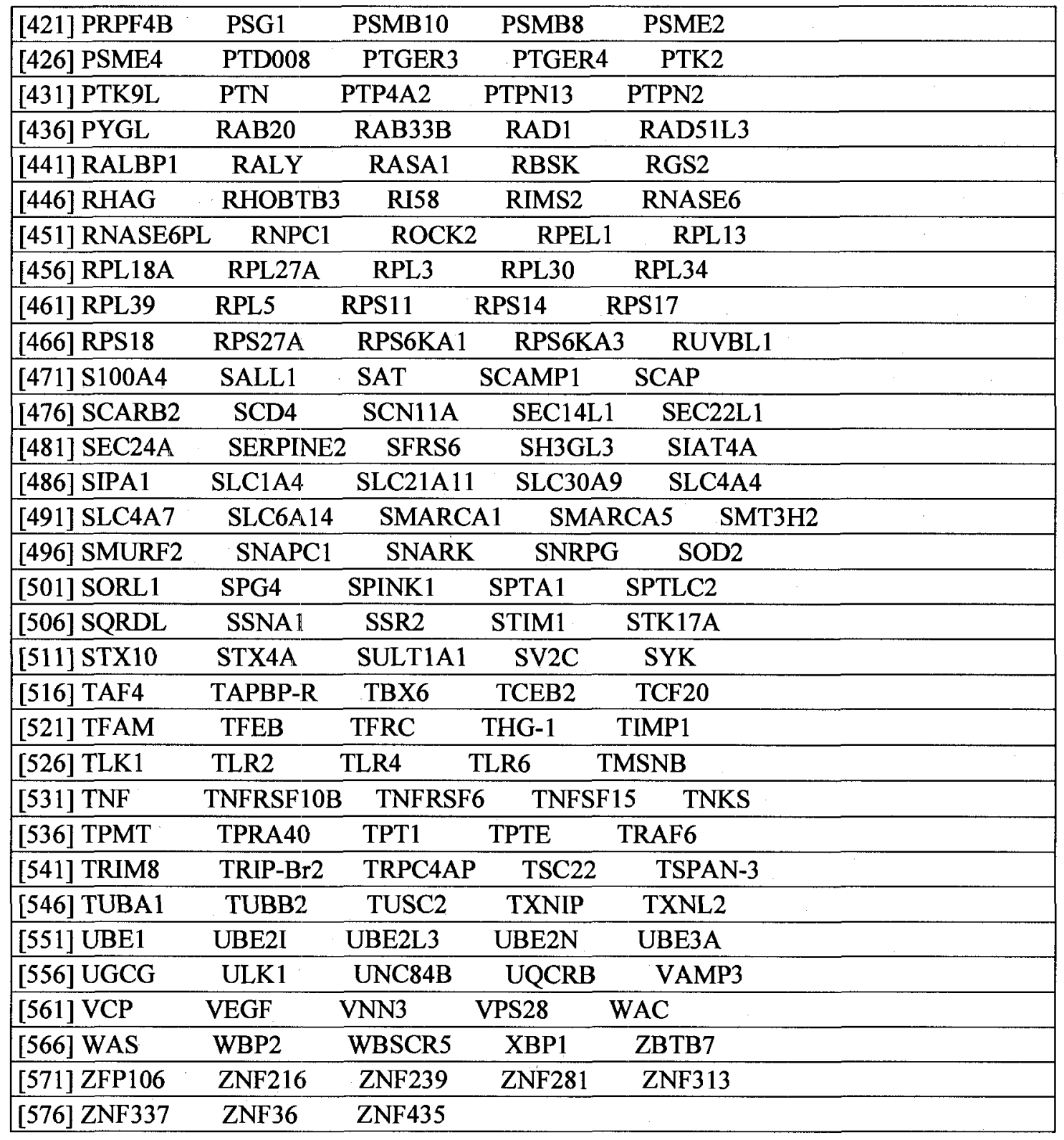


Table A.8. Those genes not in differentially regulated gene set (gene set A) but only in the response gene set under treatment (gene set $B$ ). Total 1670 genes.

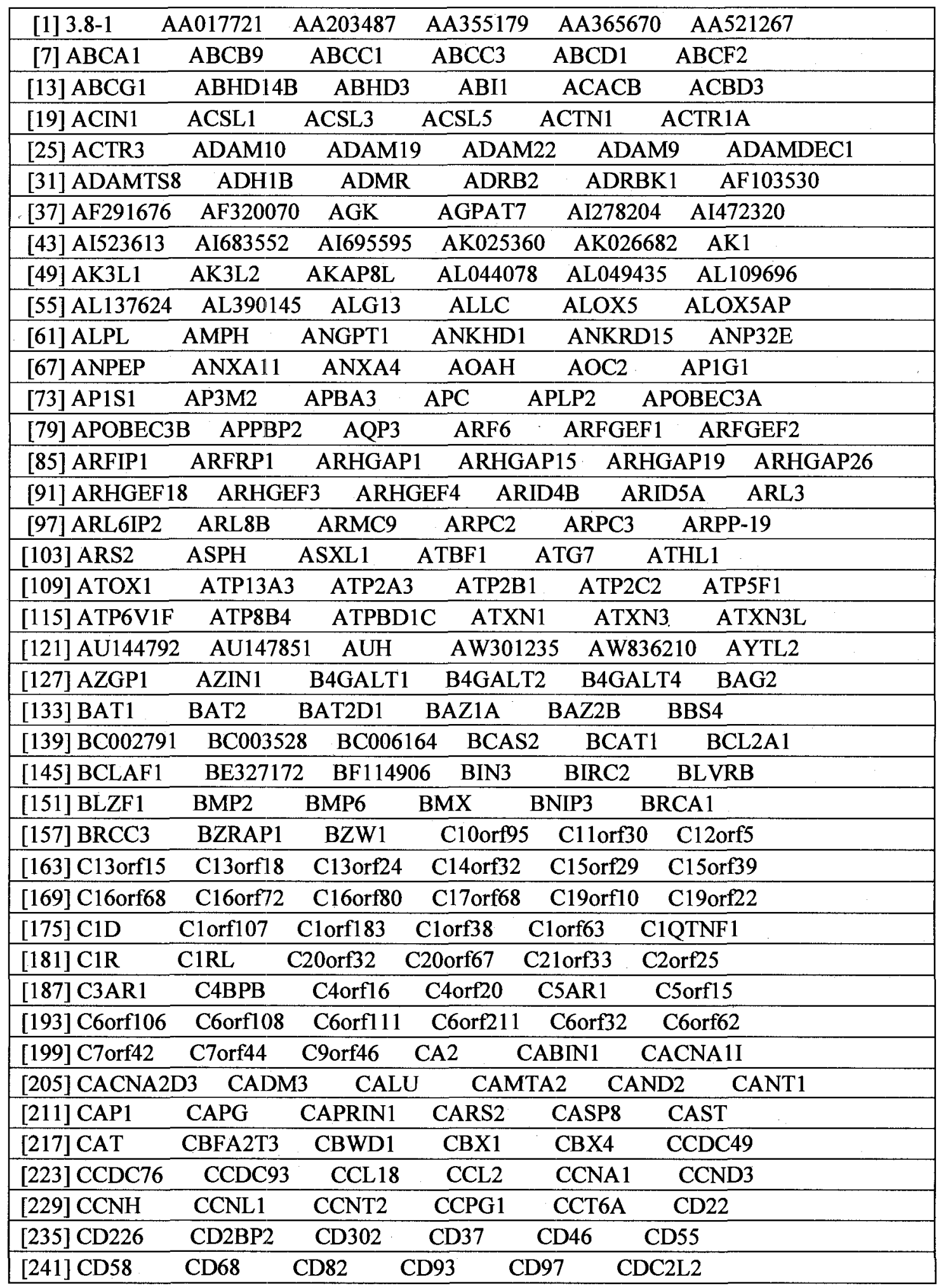




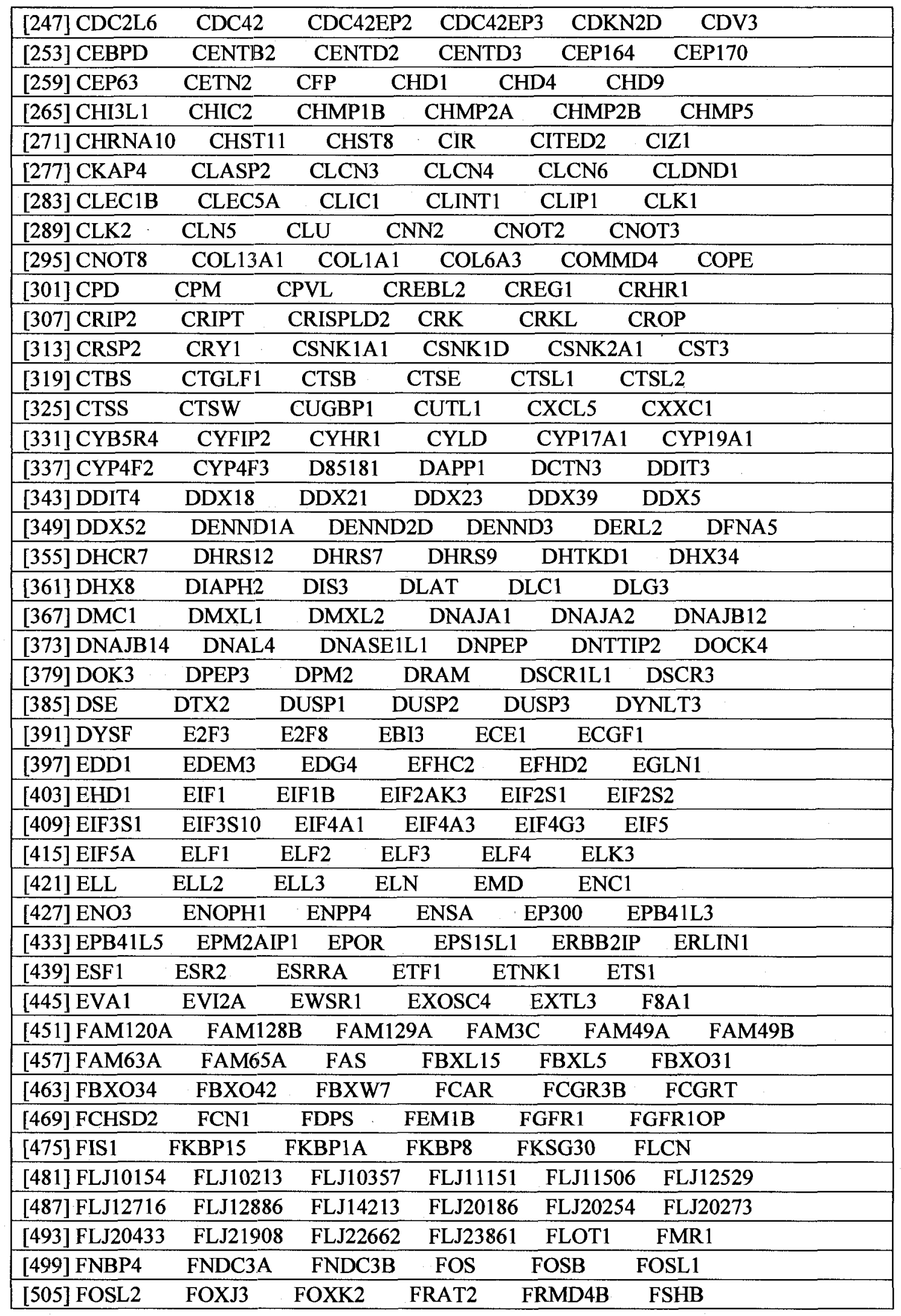




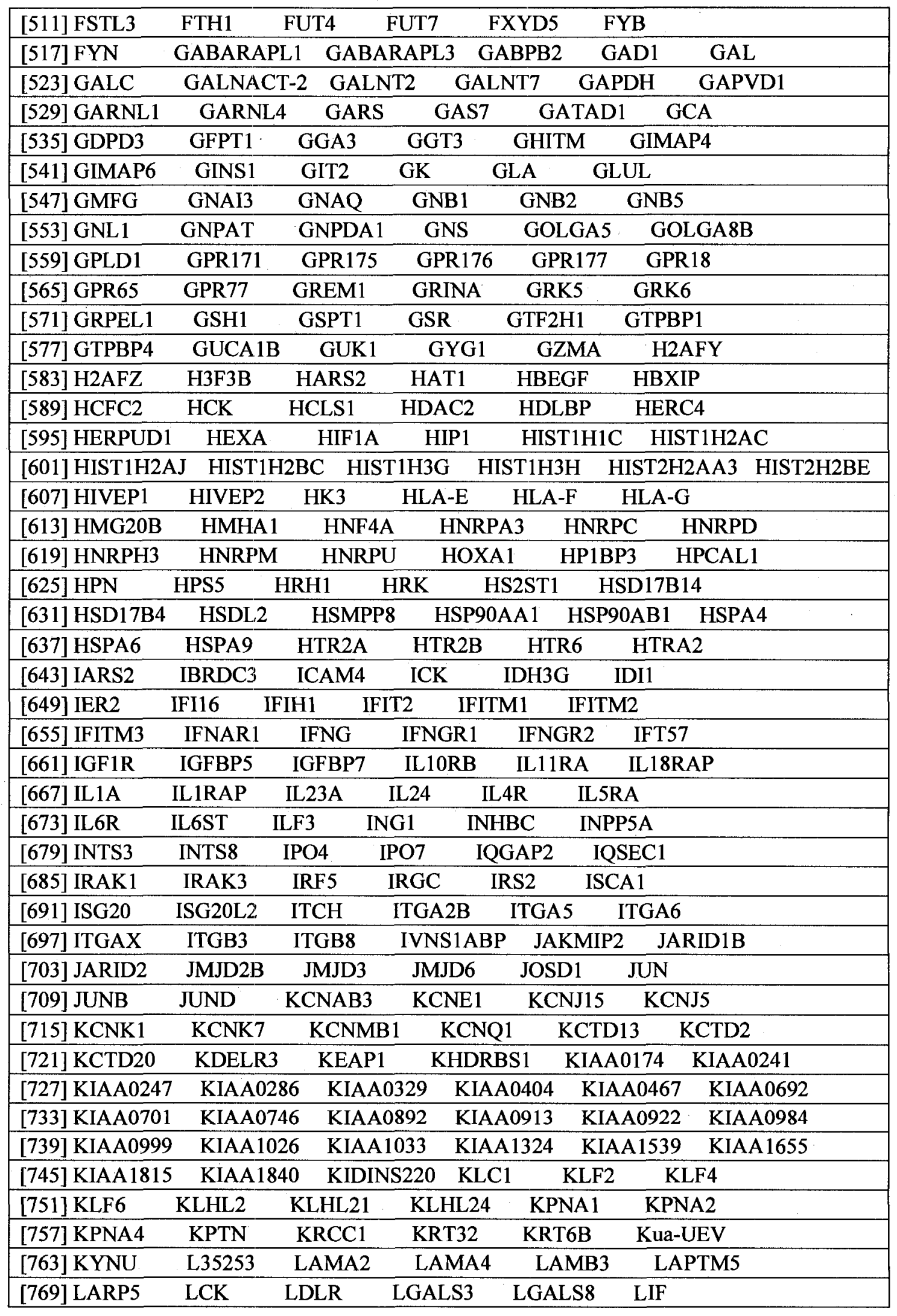




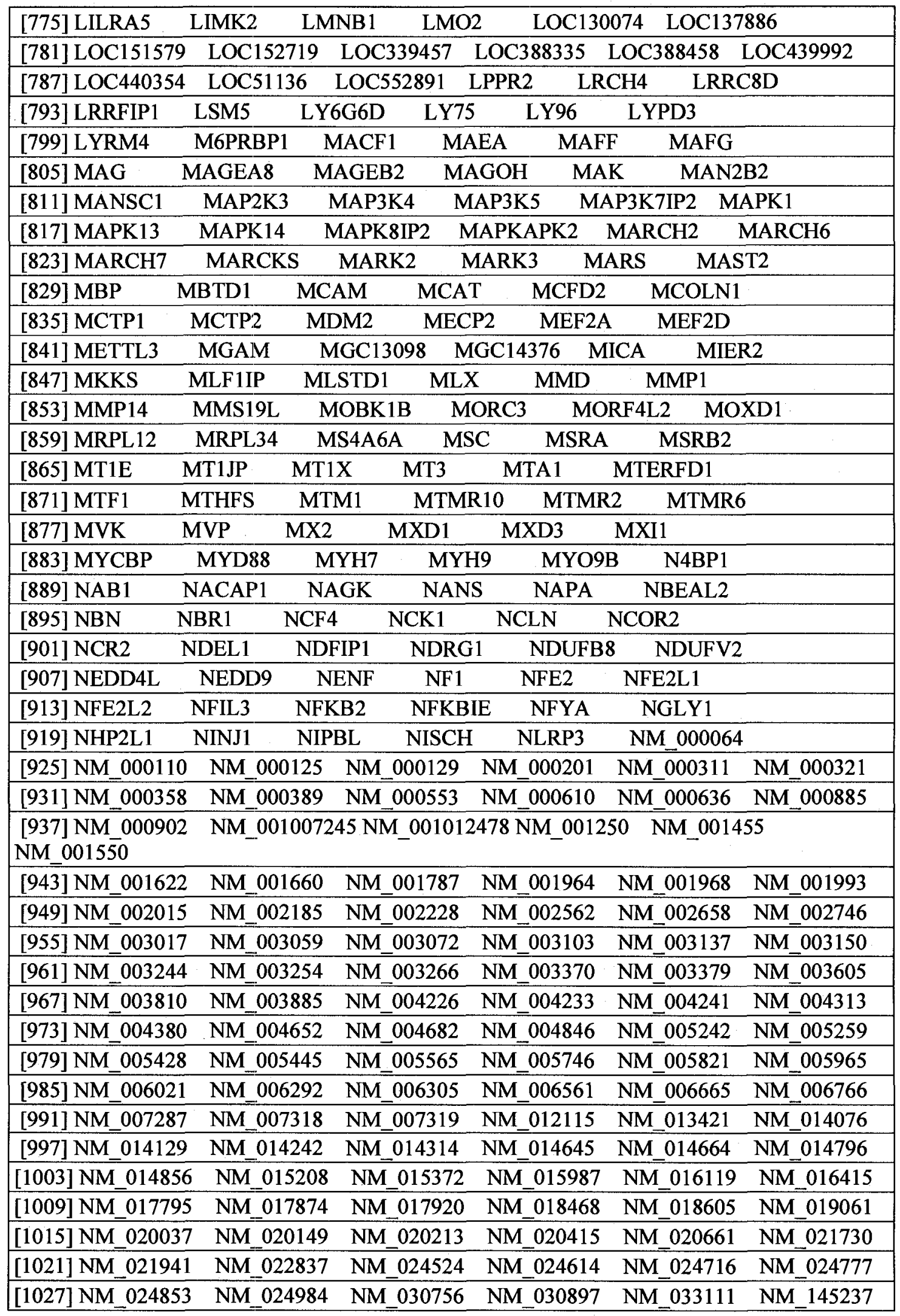




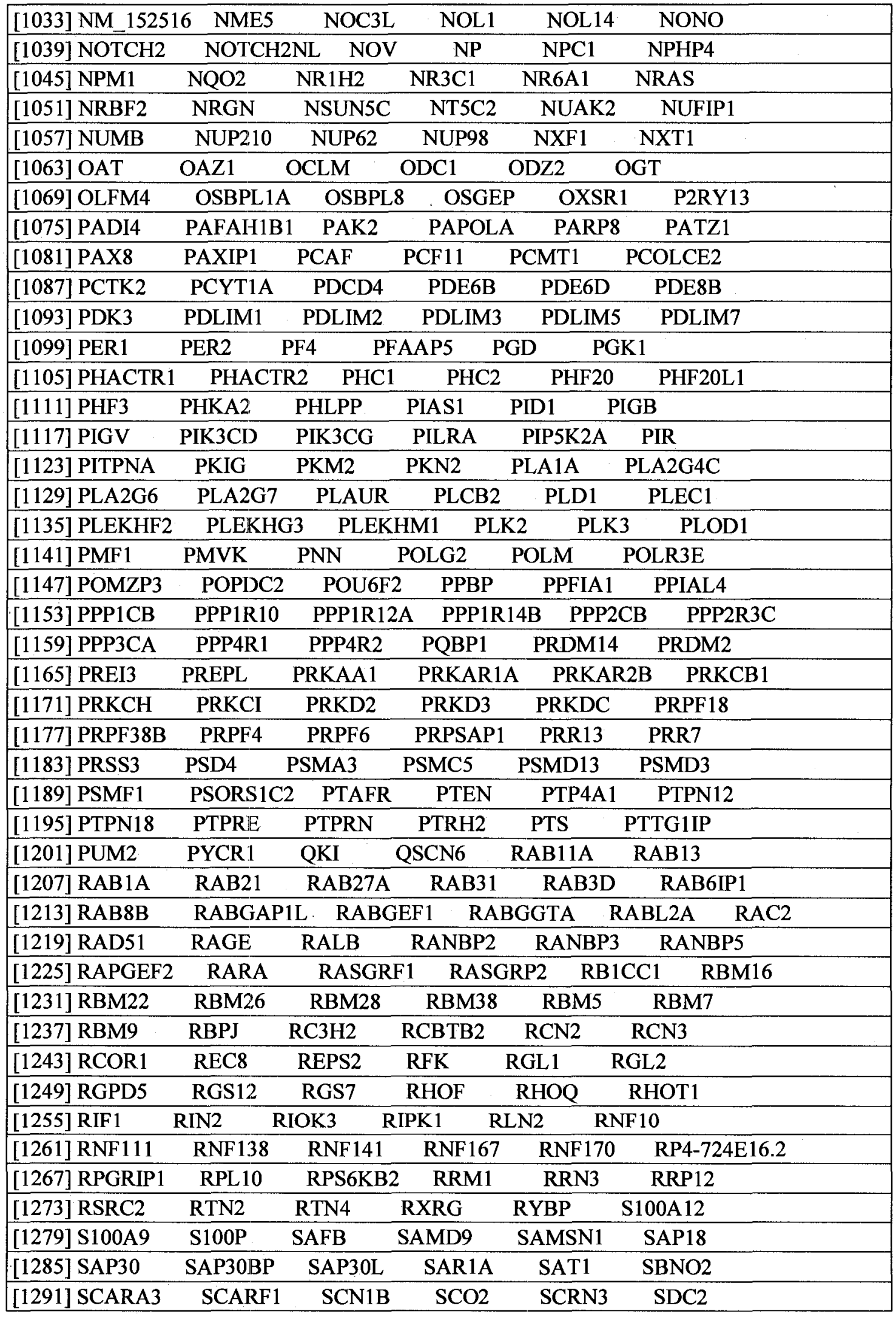




\begin{tabular}{|c|c|}
\hline [1297] SDCCAG3 & $\begin{array}{lll}\text { SEC23IP } & \text { SECISBP2 } & \text { SECTM1 }\end{array}$ \\
\hline [1303] SEL1L & SEMA6B \\
\hline [1309] SENP6 & SERPINB 1 \\
\hline [1315] SERPINB7 & $\begin{array}{llll}\text { SERPINB8 } & \text { SERPINI2 } & \text { SERTAD2 } & \text { SETX }\end{array}$ \\
\hline$[1321] \mathrm{SF} 3 \mathrm{~B} 4$ & SFRS2IP \\
\hline$[1327]$ SFRS5 & SH2D3C \\
\hline [1333] SH3GLB1 & SIGLEC7 SIRPA \\
\hline [1339] SKAP2 & SLAMF7 $\quad$ SLC11A1 \\
\hline [1345] SLC14A2 & SLC19A2 \\
\hline$[1351]$ SLC25A32 & SLC25A37 SLC25A44 $\quad$ SLC26A2 \\
\hline [1357] SLC35A2 & SLC39A8 $\quad$ SLC3A2 \\
\hline [1363] SLC5A3 & SLCO4A1 \\
\hline [1369] SLCO4C1 & SMARCE1 $\quad$ SMCHD1 \\
\hline [1375] SMPD2 & $\begin{array}{llll}\text { SNIP1 } & \text { SNRPA1 } & \text { SNRPB } & \text { SNTB2 }\end{array}$ \\
\hline [1381] SNX1 & SNX10 \\
\hline$[1387] \mathrm{SON}$ & SPAG5 \\
\hline [1393] SPAG9 & SPATA5L1 SPATA6 \\
\hline [1399] SPINT1 & $\begin{array}{lll}\text { SPOCK2 } & \text { SPTB } & \text { SPTBN1 }\end{array}$ \\
\hline [1405] SRC & SRGAP3 \\
\hline [1411] SSFA2 & ST3GAL2 \\
\hline [1417] ST8SIA4 & STARD8 \\
\hline [1423] STAT5B & STMN1 \\
\hline [1429] STX16 & $\begin{array}{ll}\text { STX7 } & \text { STXBP2 }\end{array}$ \\
\hline [1435] SUCLG1 & $\begin{array}{llll}\text { SUPT6H } & \text { SUPV3L1 } & \text { SVIL } & \text { SYCP2 }\end{array}$ \\
\hline [1441] SYNCRIP & SYNE2 \\
\hline [1447] TAGLN2 & TARDBP \\
\hline [1453] TBC1D22A & A $\quad$ TBC1D2B \\
\hline [1459] TDG & $\begin{array}{llll}\text { TES } & \text { TFDP1 } & \text { TFR2 } & \text { TGFBR3 }\end{array}$ \\
\hline$[1465]$ TGIF2 & THRAP1 \\
\hline [1471] THRAP5 & TIMM17A TIPARP \\
\hline$[1477]$ TLN1 & TM9SF3 \\
\hline$[1483] \mathrm{TMCO} 3$ & TMEM110 $\quad$ TMEM165 \\
\hline [1489] TMEM24 & TMEM41B \\
\hline [1495] TMEPAI & TNFRSF1B TNFRSF8 \\
\hline$[1501]$ TOB 1 & TOMM20 \\
\hline [1507] TOR1AIP1 & TPD52L2 \\
\hline [1513] TRAC & TRAF3IP3 TRAPPC3 \\
\hline [1519] TREM1 & TREML2 \\
\hline [1525] TRIM27 & TRIM36 \\
\hline [1531] TSC22D2 & TSPAN3 \\
\hline [1537] TTC19 & TUBA1A \\
\hline$[1543]$ TUBA $1 C$ & TXNDC13 \\
\hline [1549] U56725 & UBAP2L UBE2B \\
\hline [1555] UBE2D3 & UBE2E1 \\
\hline
\end{tabular}




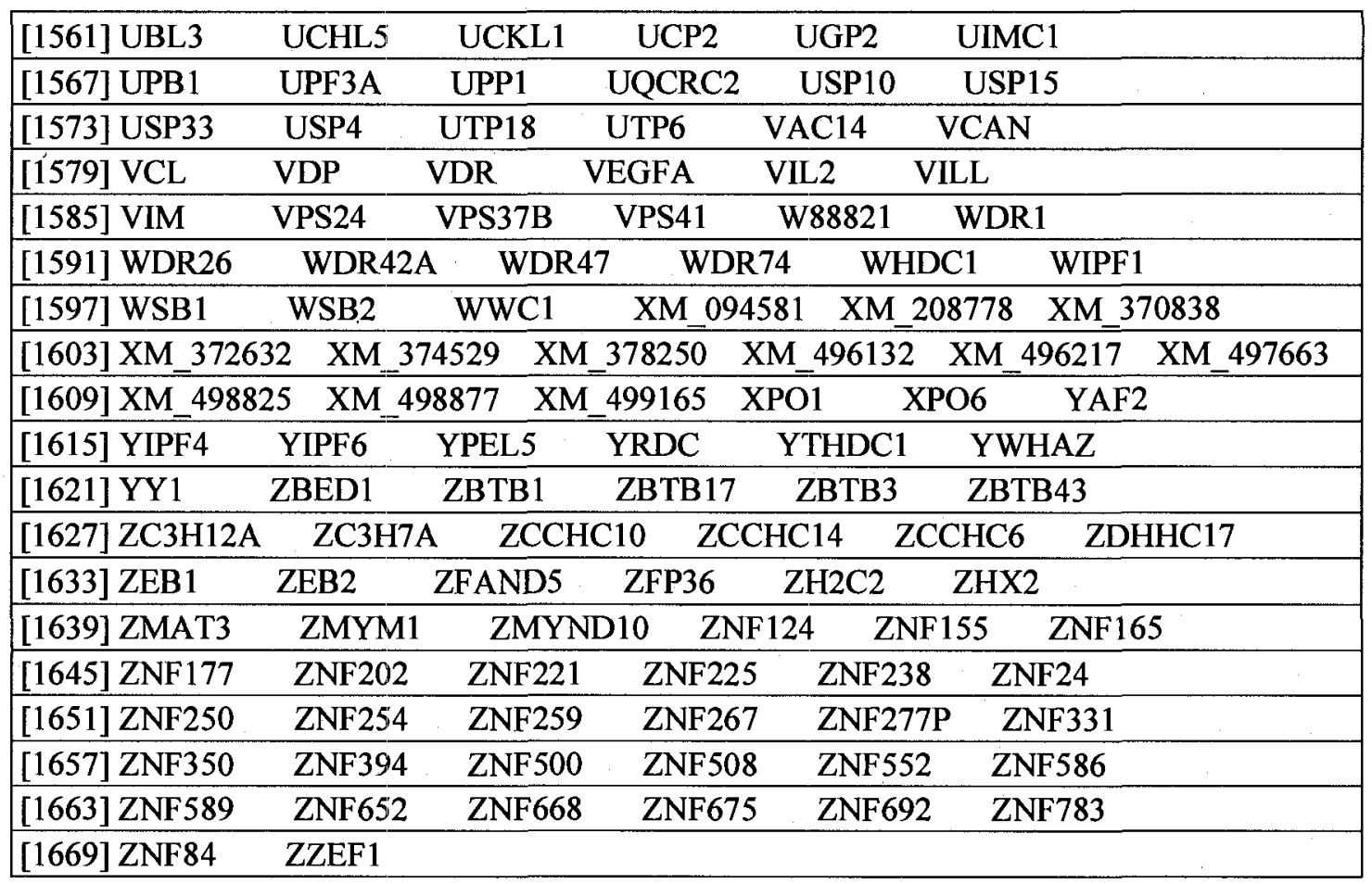


Table A.9. Those genes in both differentially regulated gene set (gene set A) and in the response gene set under treatment (gene set $\mathrm{C}$ ). Total 80 genes.

\begin{tabular}{|lccccccccc|}
\hline [1] ABHD5 & ADD1 & AMD1 & ANXA5 & ARTS-1 & BRD8 & BTG3 & BTN3A1 \\
\hline [9] C14orf159 & C20orf121 & CAMK2G & CCL19 & CCL3 & CCL4 & CD48 & CD59 \\
\hline [17] CD69 & CIB1 & CLCN7 & CLIC4 & COX5B & CREB5 & CSAD & CSTB \\
\hline [25] DUSP10 & FABP5 & FLI1 & GALNT3 & GCLM & GGT1 & GMFB & IL12B \\
\hline [33] IL1F9 & INHBA & INPP4A & ISGF3G & ITM2B & ITPR1 & JAG1 & KIAA0143 \\
\hline [41] KIF1B & LIMS1 & LOC54103 & M6PR & MAN1A1 & MATR3 & MCM4 & MGLL \\
\hline [49] MPZL1 & ORM1 & P2RX4 & PDPK1 & PGCP & PGLS & PLSCR4 & PSMA6 \\
\hline [57] PSMD12 & PSMD4 & RAD21 & RAD23B & RERE & RNPEP & RPS6KA5 & RPS9 \\
\hline [65] S100A6 & SEC23B & SERPINB9 & SIAH1 & SLBP & SLC43A3 & SLC7A7 & SLPI \\
\hline [73] SPG20 & SPHK1 & SPINLW1 & TBC1D2 & TKT & TNFRSF1A & TRA@ & VNN2 \\
\hline
\end{tabular}


Table A.10. Those genes only in differentially regulated gene set (gene set A) but not in the response gene set under treatment (gene set $\mathrm{C}$ ). Total 730 genes.

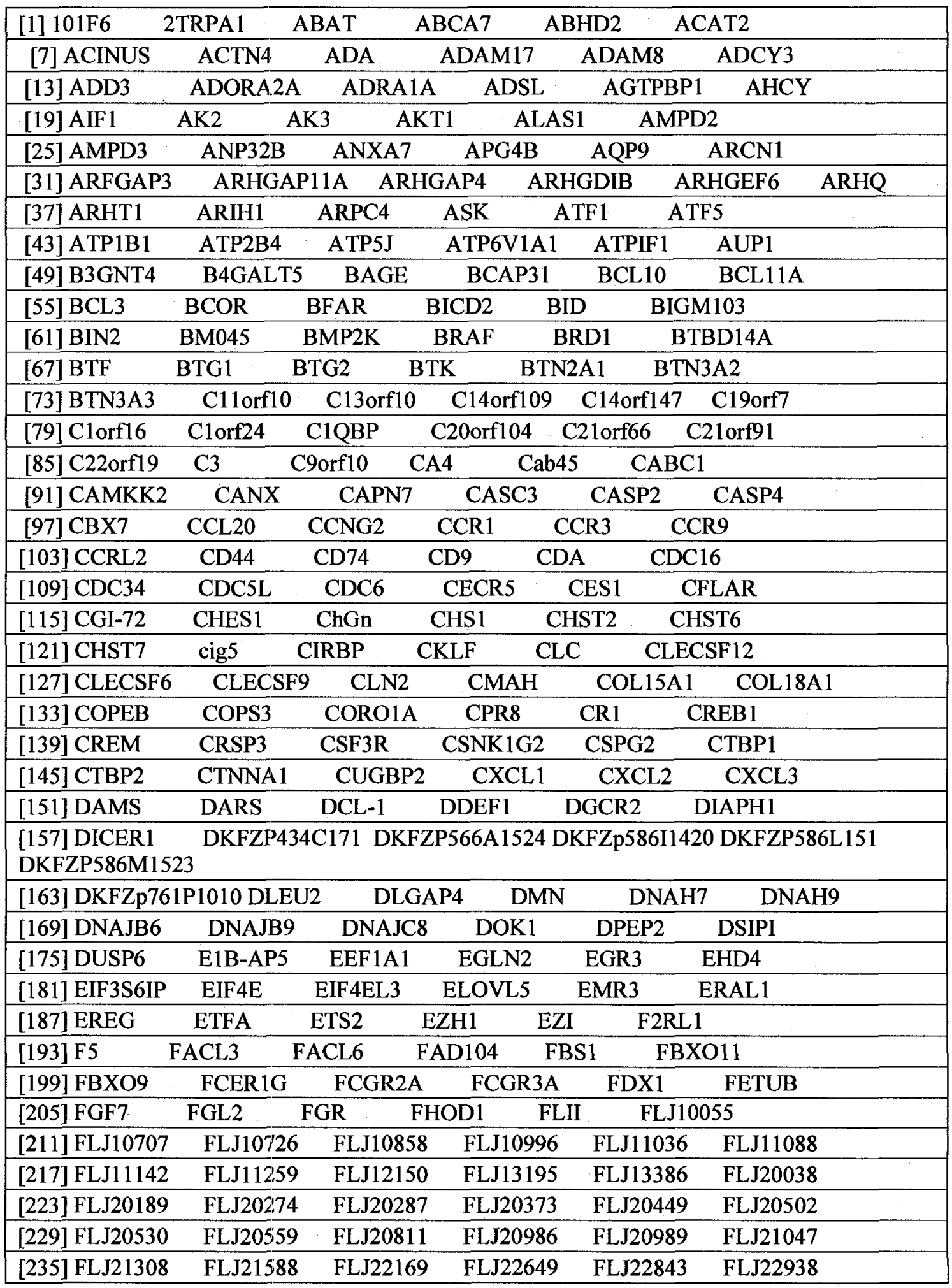




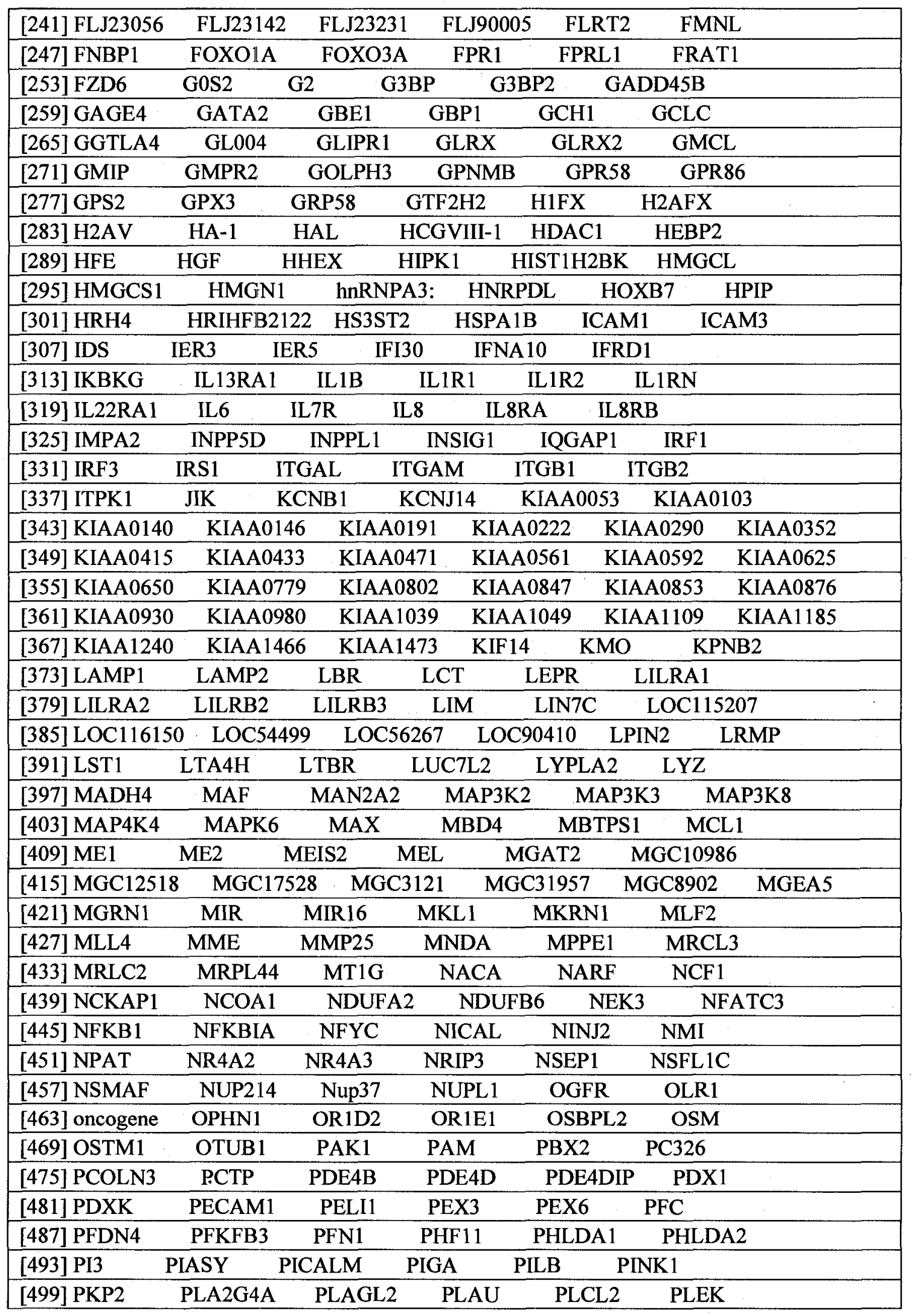




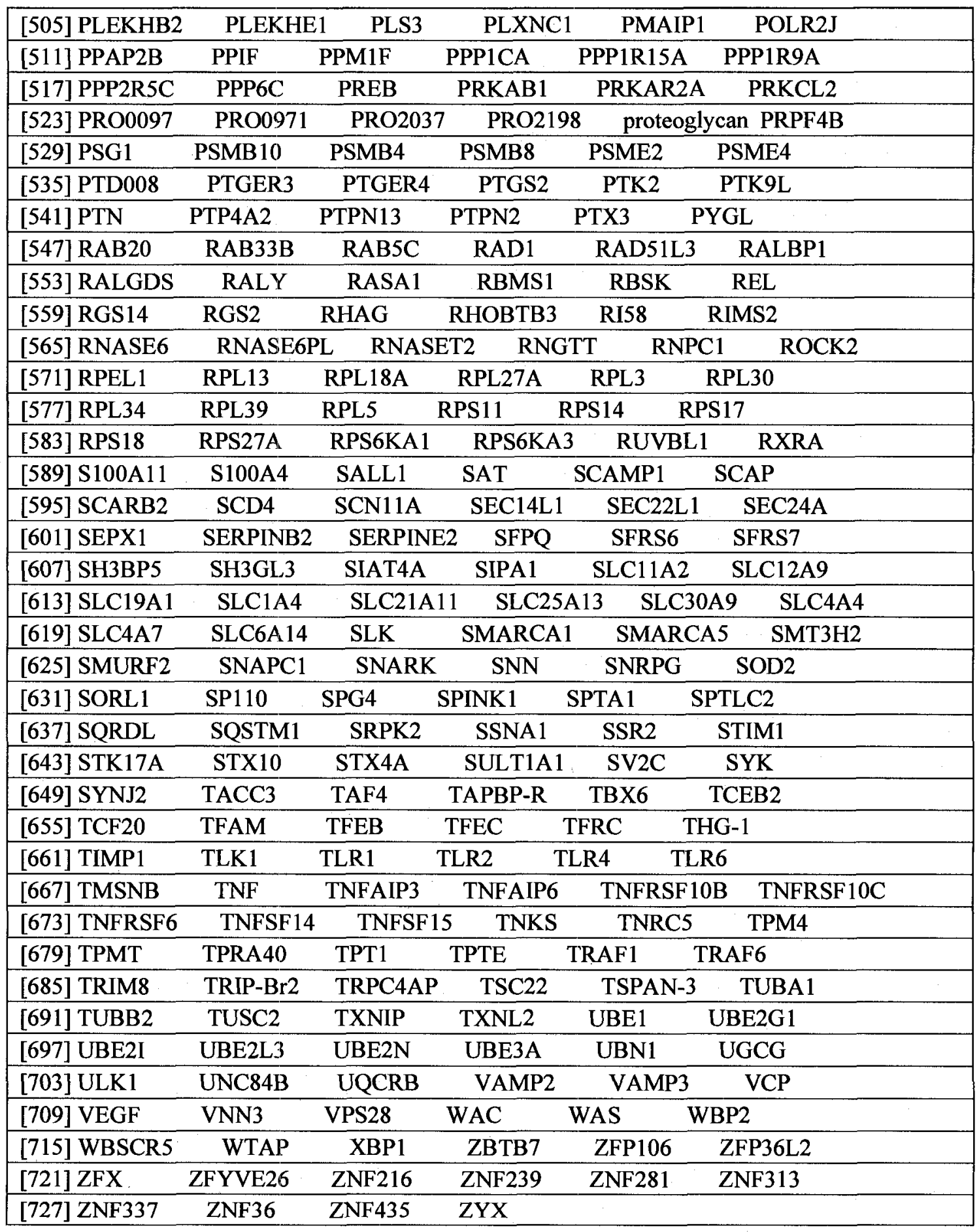


Table A.11. Those genes not in differentially regulated gene set (gene set A) but in the response gene set under treatment (gene set $C$ ). Total 928 genes.

\begin{tabular}{|c|c|}
\hline [1] 3.8-1 & AA355179 AA365670 \\
\hline [7] $\mathrm{ABCD} 1$ & ABHD14B \\
\hline [13] ACIN1 & ADAMTS8 \\
\hline [19] ADH1B & AF291676 \\
\hline [25] AGK & AI683552 AK025360 \\
\hline [31] AK026682 & AL044078 \\
\hline [37] AL049435 & AL109696 AL137624 AL390145 ALLC \\
\hline [43] ANGPT1 & ANKRD15 \\
\hline [49] AP3M2 & APOBEC3B APPBP2 \\
\hline [55] ARF6 & ARHGAP19 \\
\hline [61] ARHGEF3 & ARHGEF4 \\
\hline [67] ARPC2 & ARPP-19 \\
\hline [73] ATG7 & ATP6V1F \\
\hline [79] ATXN3 & AW301235 AW836210 \\
\hline [85] AZGP1 & B4GALT4 \\
\hline [91] BAZ2B & $\mathrm{BC} 002791 \quad \mathrm{BC} 003528$ \\
\hline [97] BLVRB & BRCA1 \\
\hline [103] BZRAP1 & C10orf95 C11orf30 C12orf5 \\
\hline$[109]$ C16orf80 & C17orf68 C19orf10 C1D \\
\hline [115] C1QTNF1 & C20orf32 C21orf33 \\
\hline [121] C4orf16 & C6orf108 C6orf62 \\
\hline [127] C7orf44 & CACNA1I $\quad$ CACNA2D3 CADM3 \\
\hline [133] CAMTA2 & CAPRIN1 \\
\hline [139] CBFA2T3 & CCDC49 \\
\hline [145] CD22 & CDC2L2 \\
\hline [151] CDC42EP2 & $2 \quad$ CEP164 \\
\hline [157] CHIC2 & CHRNA10 \\
\hline [163] CLCN3 & CLDND1 \\
\hline [169] CLK2 & CNOT8 COL13A1 COL6A3 \\
\hline [175] COMMD4 & CREBL2 \\
\hline [181] CRIPT & CSNK2A1 \\
\hline [187] CTSE & CUGBP1 \\
\hline [193] CYB5R4 & CYP19A1 CYP4F2 \\
\hline [199] DAPP1 & DDX23 \\
\hline [205] DDX52 & DENND1A \\
\hline [211] DIAPH2 & DMC1 \\
\hline [217] DMXL1 & DNAJB14 \\
\hline [223] DPEP3 & DSCR1L1 DSCR3 \\
\hline [229] DTX2 & DYNLT3 \\
\hline [235] EDG4 & EIF2S2 \\
\hline [241] EIF3S10 & EIF4G3 \\
\hline
\end{tabular}




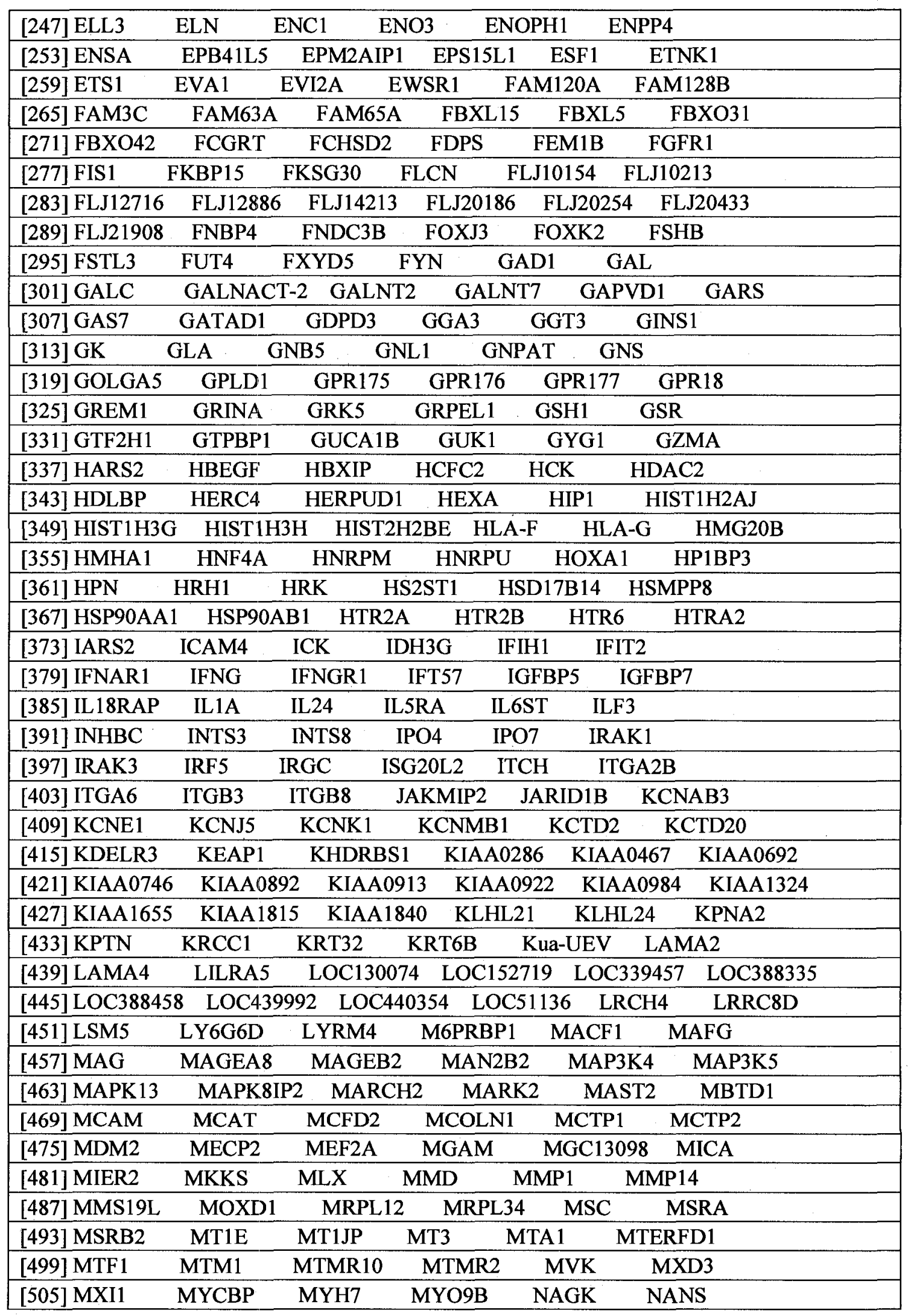




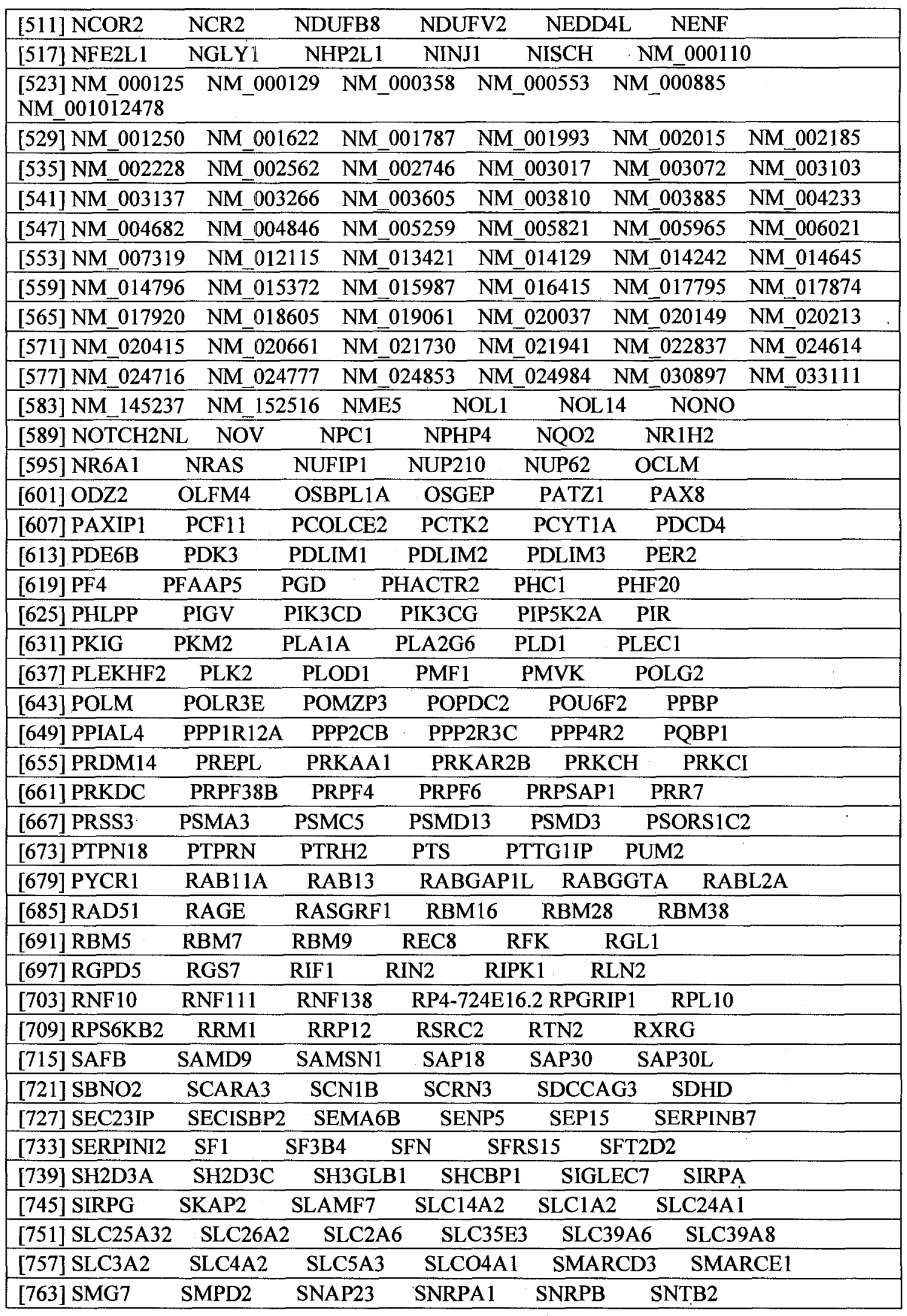




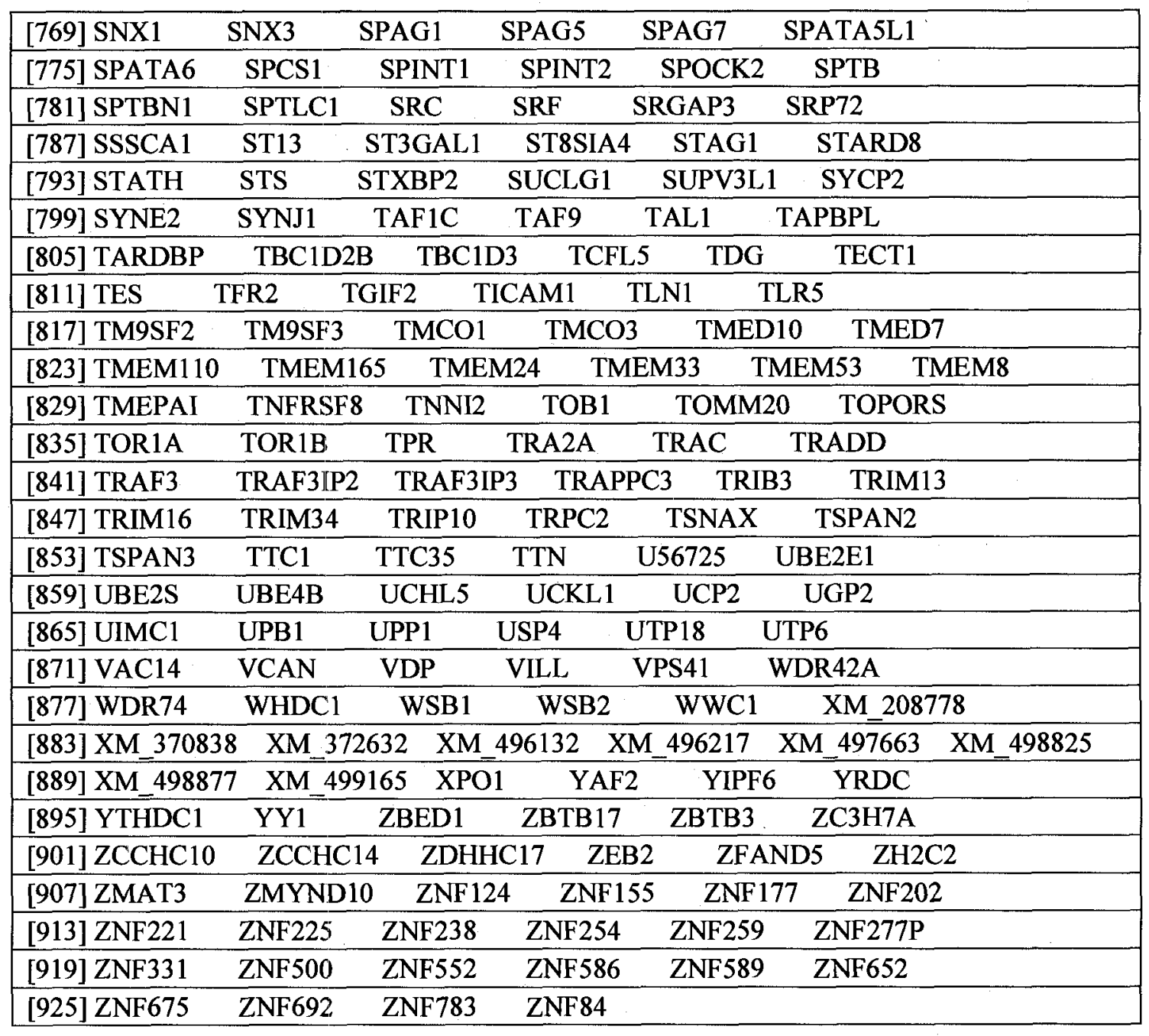




\section{Appendix B.}

\section{Computation Codes in $\mathbf{R}$ version 2.3.1 or latter:}

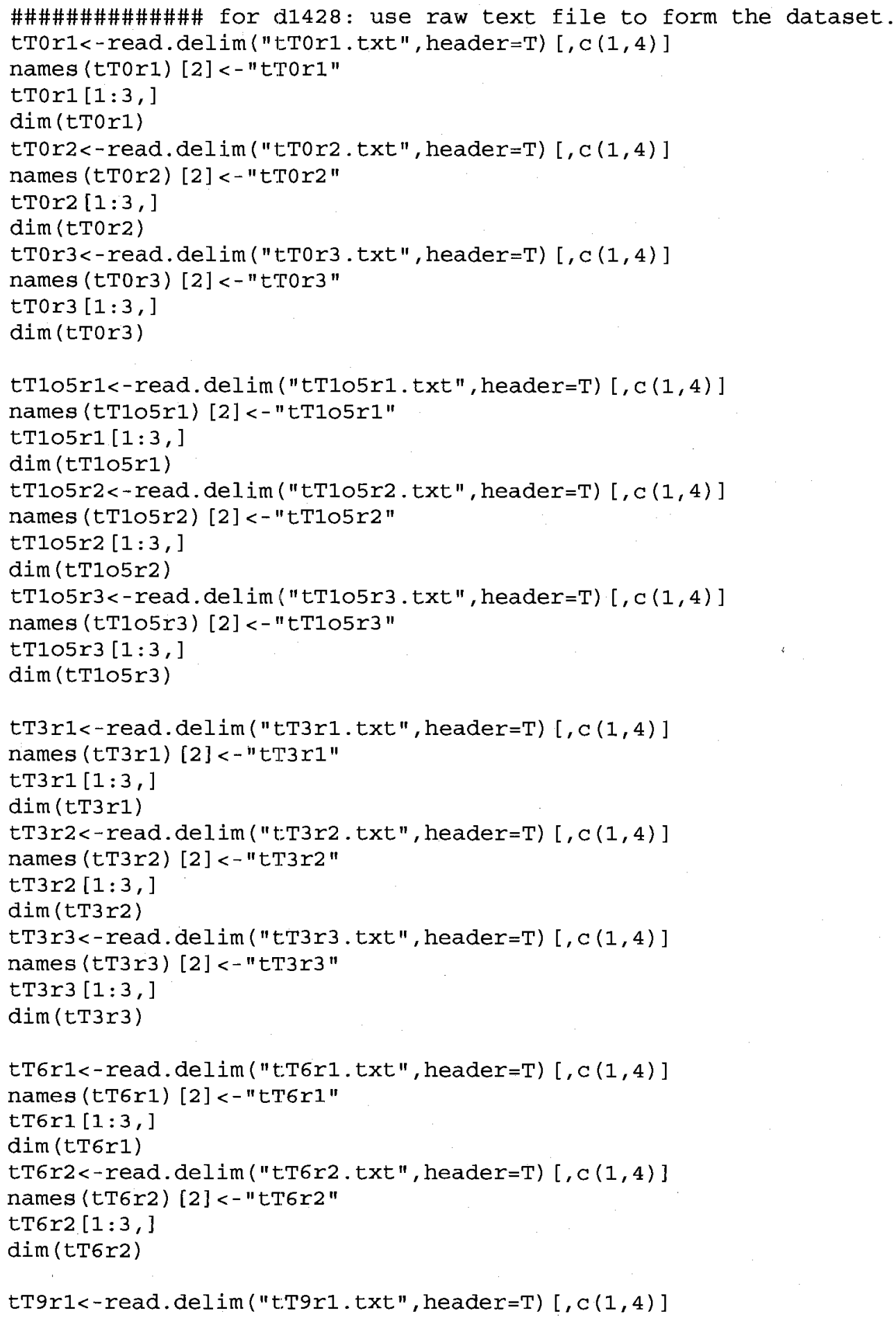




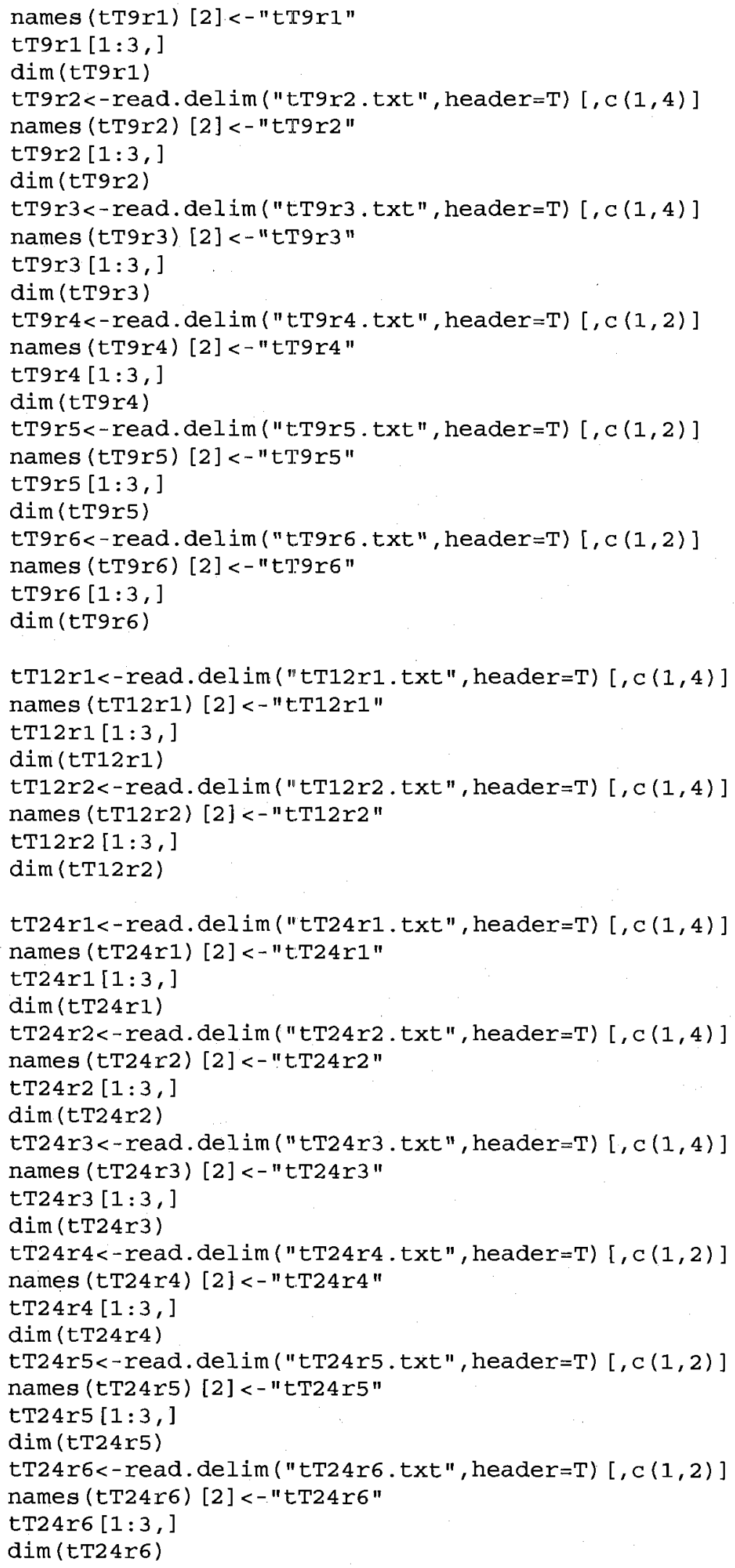




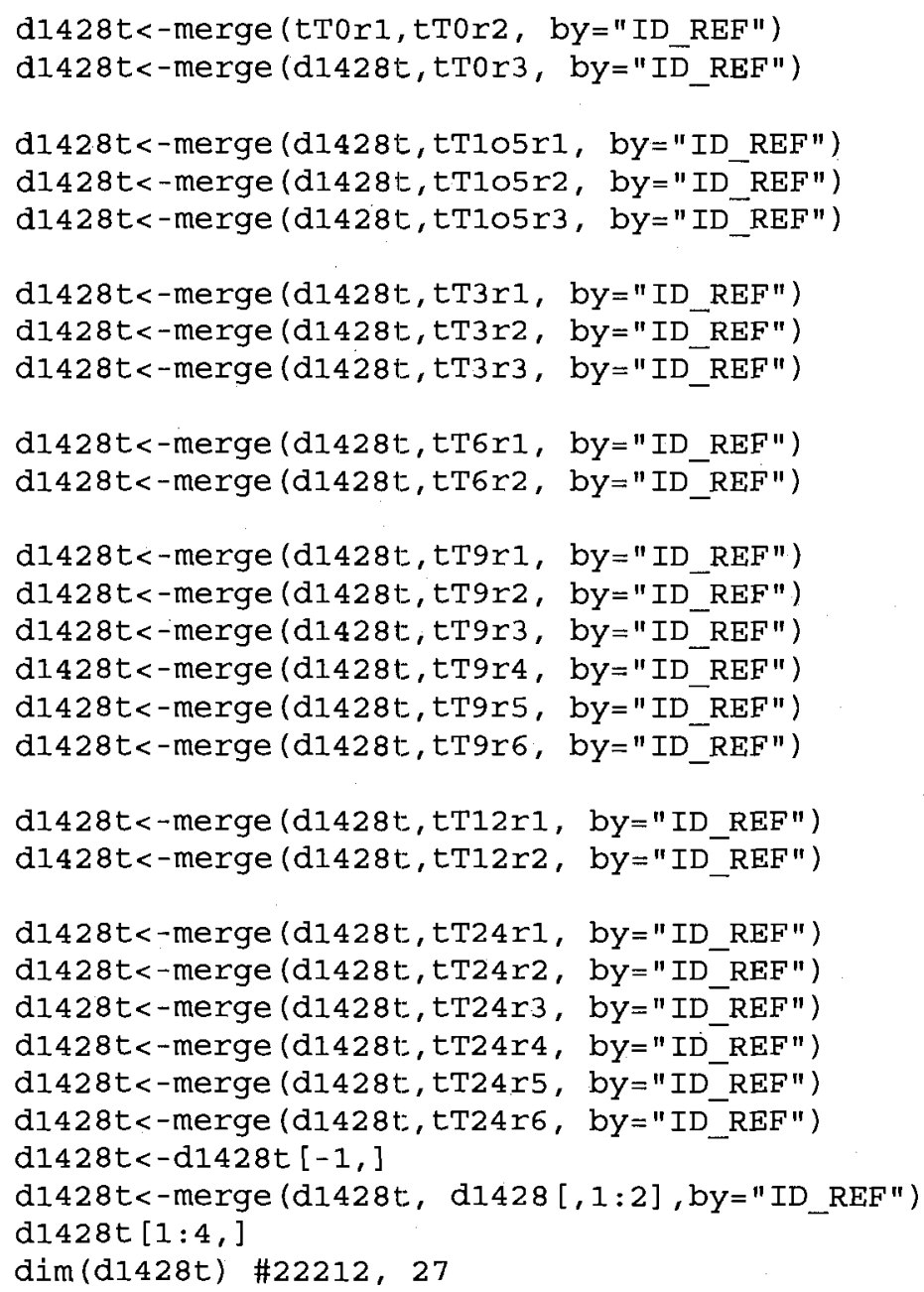

\#\#\#\#\#\#\#\#\#\#\#\#\#\#\# form d1428c dataset \#\#\#\#\#\#\#\#\#\#\#\#\# cTor $1<-r e a d . d e l i m(" c T 0 r 1 . t x t "$, header=T) $[, \mathrm{c}(1,4)]$ 


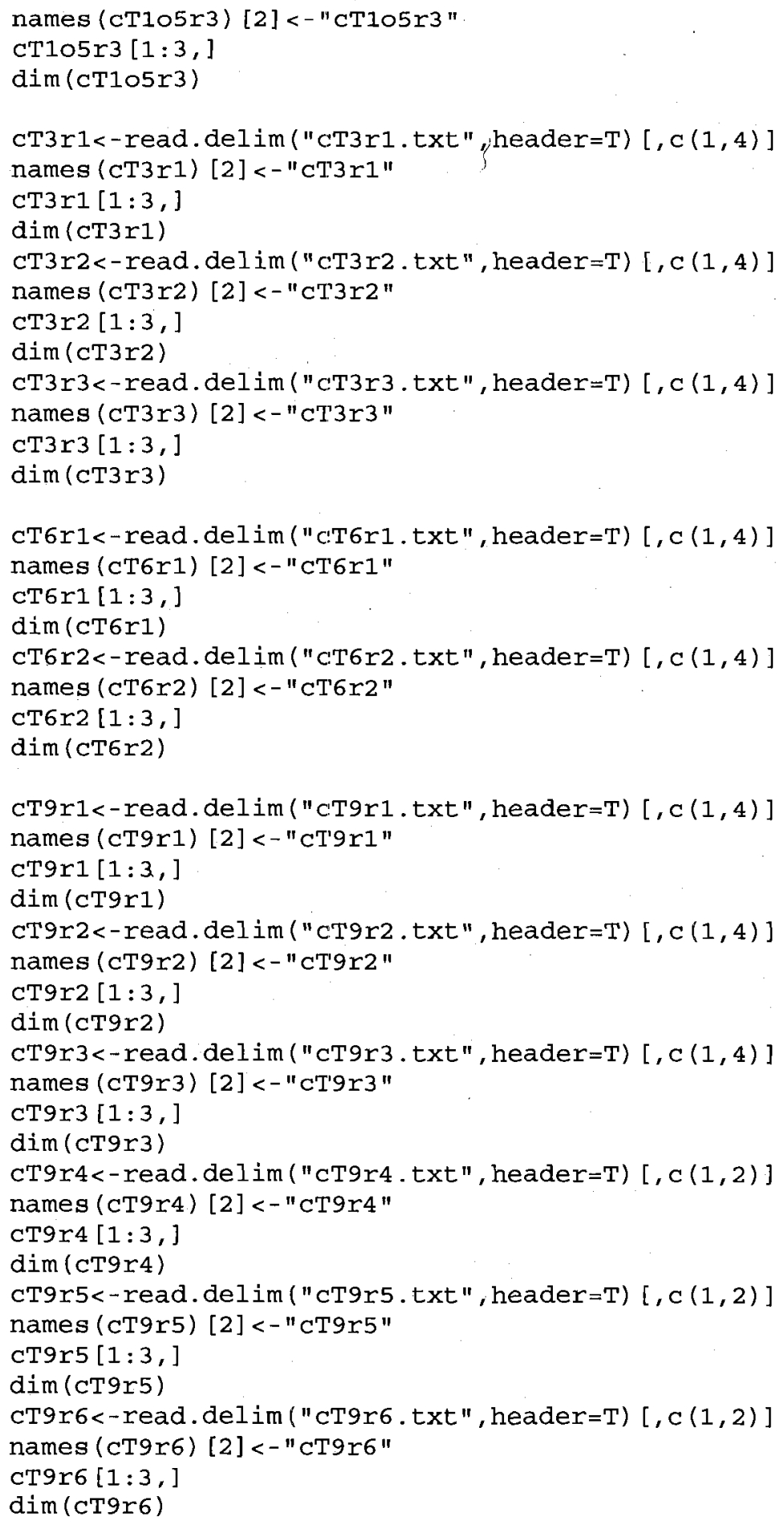




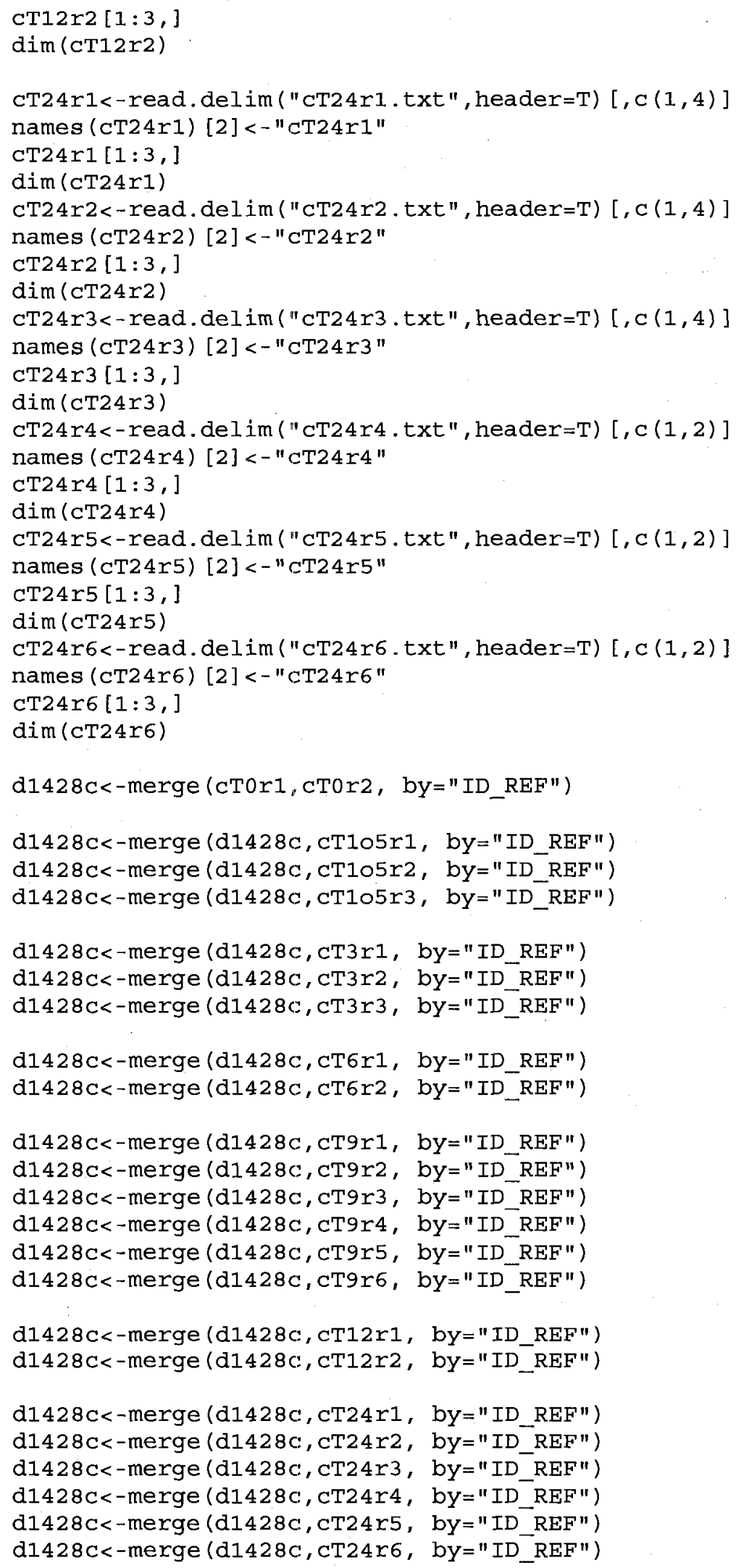


$\mathrm{d} 1428 \mathrm{c}<-\mathrm{d} 1428 \mathrm{c}[-1$,

d1428c<-merge (d1428c, d1428[, 1:2], by="ID_REF")

$\mathrm{d} 1428 \mathrm{c}[1: 4$,

$\operatorname{dim}(\mathrm{d} 1428 \mathrm{c}) \# 22214,26$

\#\#\#\# d1428c raw dataset ready \#\#\#\#\#\#\#\#\#\#\#\#\#\#\#\#\#\#\#\#\#

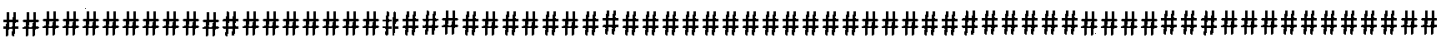
check NA value and delete

$\mathrm{x} 1<-$ numeric ()

for ( $i$ in $2: \operatorname{dim}(\mathrm{d} 1428 \mathrm{c})[2])\{$

$\mathrm{x} 1[(\mathrm{i}-1)]<-$ length (d1428C\$ID REF [is.na $(\mathrm{d} 1428 \mathrm{C}[, i])])$

\}

\#\#\#\#\#\#\#\#\#\#\#\#\#\#\#\#\#\#\#\# No NA values

\#\#\#\#\#\#\#\#\#\#\#\#\#\#\#\#\#\#\#\#\#\# Empirical Bayesian Estimation

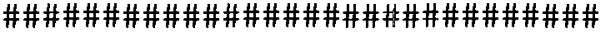

\#\#\# get mean and std for each time point

d1428c\$T0m<-apply (d1.428c [, 2:3], 1, mean)

d1428c\$T0sd<-apply (d1428c [, 2:3], 1, sd)

d1428c\$T105m<-apply (d1428c [,4:6], 1, mean)

d1428c\$T105sd<-apply (d1428c [, 4:6], 1, sd)

d1428c\$T3m<-apply (d1.428C [, 7:9], 1, mean)

d1428c\$T3sd<-apply (d1428c [, 7:9], 1, sd)

d1428c\$T6m<-apply (d1428c [, 10:11], 1, mean)

d1428c\$T6sd<-apply (d1428c [, 10:11], 1, sd)

d1428c\$T9m<-apply (d1428c [, 12:17], 1, mean)

d1428c\$T9sd<-apply (d1428c [, 12:17], 1, sd)

d1428c\$T12m<-apply (d1428c [, 18:19], 1, mean)

d1428c\$T12sd<-apply (d1428c [, 18:19], 1, sd)

d1428c\$T24m<-apply (d1428c [ , $20: 25], 1$, mean)

d1428c\$T24sd<-apply (d1428c [, 20:25],1, sd)

d1428t\$T0m<-apply (d1428t $[, 2: 4], 1$, mean)

d1428t \$T0sd<-apply (d1428t [, 2:4], 1, sd)

d1428t\$T105m<-apply (d1428t $[, 5: 7], 1$, mean)

d1428t $\$$ T105sd<-apply (d1428t $[, 5: 7], 1, \mathrm{sd})$

d1428t $\$$ T 3 m $<-\operatorname{apply~(d1428t~}[, 8: 10], 1$, mean)

d1428t\$T3sd<-apply (d1428t $[, 8: 10], 1, \mathrm{sd})$

d1428t\$T6m<-apply (d1428t $[, 11: 12], 1$, mean)

d1428t\$T6sd<-apply (d1428t $[, 11: 12], 1$, sd)

d1428t\$T9m<-apply (d1428t $[, 13: 18], 1$, mean)

d1428t\$T9sd<-apply (d1428t $[, 13: 18], 1, \mathbf{s d})$

d1428t $\$ T 12 m<-a p p l y(d 1428 t[, 19: 20], 1$, mean)

d1428t\$T12sd<-apply (d1428t $[, 19: 20], 1, \mathrm{sd})$

d1428t\$T24m<-apply (d1428t [,21:26], 1, mean)

d1428t\$T24sd<-apply (d1428t [,21:26],1, sd)

\#\#\#\#\#\# check sd equals to zero rows, make the row number the same for each experiment

$\operatorname{dim}(\mathrm{d} 1428 \mathrm{c}[\mathrm{d} 1428 \mathrm{c} \$ \mathrm{~T} 0 \mathrm{sd}==0|\mathrm{~d} 1428 \mathrm{c} \$ \mathrm{~T} 105 \mathrm{sd}==0| \mathrm{d} 1428 \mathrm{c} \$ \mathrm{~T} 3 \mathrm{sd}==0 \mid \mathrm{d} 1428 \mathrm{c} \$ \mathrm{~T} 6 \mathrm{sd}==0$ $|\mathrm{d} 1428 \mathrm{c} \$ \mathrm{~T} 9 \mathrm{sd}==0| \mathrm{d} 1428 \mathrm{c} \$ \mathrm{~T} 12 \mathrm{sd}==0 \mid$

$\mathrm{d} 1428 \mathrm{c} \$ \mathrm{~T} 24 \mathrm{sd}==0$, ] ) \# 240 rows

$\mathrm{d} 1428 \mathrm{c}[\mathrm{d} 1428 \mathrm{c} \$ \mathrm{~T} 0 \mathrm{sd}==0|\mathrm{~d} 1428 \mathrm{c} \$ \mathrm{~T} 105 \mathrm{sd}==0| \mathrm{d} 1428 \mathrm{c} \$ \mathrm{~T} 3 \mathrm{sd}==0|\mathrm{~d} 1428 \mathrm{c} \$ \mathrm{~T} 6 \mathrm{sd}==0| \mathrm{d} 14$ $28 \mathrm{c} \$ \mathrm{~T} 9 \mathrm{sd}==0|\mathrm{~d} 1428 \mathrm{c} \$ \mathrm{~T} 12 \mathrm{sd}==0|$

d1428c\$T24sd==0, ] [ , 1] 
$\operatorname{dim}(\mathrm{d} 1428 \mathrm{c}[\mathrm{d} 1428 \mathrm{c} \$ \mathrm{TO}$ sd $!=0 \& \mathrm{~d} 1428 \mathrm{c} \$ \mathrm{~T} 105 \mathrm{sd} !=0 \&$ d1428c\$T3sd! $=0 \&$ d1428c\$T6sd!=0 \& d1428c\$T9sd!=0

\& d1428c\$T12sd!=0 \& d1428c\$T24sd!=0,]) \# 21974, 40

$\operatorname{dim}(\mathrm{d} 1428 \mathrm{c})$ \# 22214,40

d1428c1<-d1428c [d1428c\$T0sd!=0 \& d1428c\$T105sd!=0 \& d1428C\$T3sd!=0 \&

d1428c\$T6sd!=0 \& d1428c\$T9sd!=0

$\& \mathrm{~d} 1428 \mathrm{C} \$ \mathrm{~T} 12 \mathrm{sd} !=0 \& \mathrm{~d} 1428 \mathrm{C} \$ \mathrm{~T} 24 \mathrm{sd} !=0$,

$\operatorname{dim}(\mathrm{d} 1428 \mathrm{t}[\mathrm{d} 1428 \mathrm{t} \$ \mathrm{TO} \mathrm{sd}==0|\mathrm{~d} 1428 \mathrm{t} \$ \mathrm{~T} 105 \mathrm{sd}==0| \mathrm{d} 1428 \mathrm{t} \$ \mathrm{~T} 3 \mathrm{sd}==0 \mid \mathrm{d} 1428 \mathrm{t} \$ \mathrm{~T} 6 \mathrm{sd}==0$

$|\mathrm{d} 1428 \mathrm{t} \$ \mathrm{~T} 9 \mathrm{sd}==0| \mathrm{d} 1428 \mathrm{t} \$ \mathrm{~T} 12 \mathrm{sd}==0 \mid$

d1428t $\$$ T24sd==0, ])

\# 203 rows

$\mathrm{d} 1428 \mathrm{t}[\mathrm{d} 1428 \mathrm{t} \$ \mathrm{TO} \mathrm{sd}==0|\mathrm{~d} 1428 \mathrm{t} \$ \mathrm{~T} 105 \mathrm{sd}==0| \mathrm{d} 1428 \mathrm{t} \$ \mathrm{~T} 3 \mathrm{sd}==0|\mathrm{~d} 1428 \mathrm{t} \$ \mathrm{~T} 6 \mathrm{sd}==0| \mathrm{d} 14$

$28 \mathrm{t} \$ \mathrm{~T} 9 \mathrm{sd}==0|\mathrm{~d} 1428 \mathrm{t} \$ \mathrm{~T} 12 \mathrm{sd}==0|$

d1428t $\$$ T2 $4 \mathrm{sd}==0],[, 1]$

$\operatorname{dim}(\mathrm{d} 1428 \mathrm{t}[\mathrm{d} 1428 \mathrm{t} \$ \mathrm{TO}$ sd $!=0 \&$ d1428t\$T105sd! $=0 \&$ d1428t\$T3sd!=0 \&

d1428t\$T6sd!=0 \& dl4:28t\$T9sd!=0

\& d1428t\$T12sd!=0 \& d1428t\$T24sd!=0,]) \#22009, 40

$\operatorname{dim}(\mathrm{d} 1428 \mathrm{t})$ \# 22212,40

d1428t1<-d1428t [d1428t\$T0sd!=0 \& d1428t\$T105sd!=0\& d1428t\$T3sd!=0\&

d1428t\$T6sd!=0\& d1428t\$T9sd!=0

\& d1428t\$T12sd!=0 \& d1428t\$T24sd!=0,]

d1428c1<-d1428c1 [d1428c1\$ID REF\%in\%ints, ]

d1428t $1<-d 1428 t 1\left[d 1428 t 1 \$ I D \_R E F \%\right.$ in $\%$ ints, ]

\#\#\#\#\#\#\#\#\#\#\#\#\#\#\#\#\# use d1428c1, d1428t1 hereafter

\#\#\#\#\#\#\#\#\#\#\#\#\#\#\#\#\#\#\#\#\#\#\#

$\#$

\# EB computation for d1428c1, d1428t1.

\#

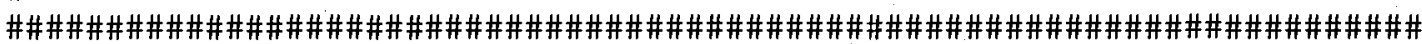

\#\#\# hyperparameter estimation $f i$ is the shape parameter in gamma dist. also call it alfa,

\#\#\# it is the squared stability measure.

fic28<-c (d1428c1\$TOm^2/d1428C1\$T0sd^2, d1428c1\$T105m^2/d1428c1\$T105sd^2, d1428c1\$T $3 \mathrm{~m}^{\wedge} 2 / \mathrm{d} 1428 \mathrm{c} 1 \$ \mathrm{~T} 3 \mathrm{sd}^{\wedge} 2, \mathrm{~d} 1428 \mathrm{c} 1 \$ \mathrm{~T} 6 \mathrm{~m}^{\wedge} 2 / \mathrm{d} 1428 \mathrm{c} 1 \$ \mathrm{~T} 6 \mathrm{sd}^{\wedge} 2$, $\mathrm{d} 1428 \mathrm{c} 1 \$ \mathrm{~T} 9 \mathrm{~m}^{\wedge} 2 / \mathrm{d} 1428 \mathrm{c} 1 \$ \mathrm{~T} 9 \mathrm{sd}^{\wedge} 2, \mathrm{~d} 1428 \mathrm{c} 1 \$ \mathrm{~T} 12 \mathrm{~m}^{\wedge} 2 / \mathrm{d} 1428 \mathrm{c} 1 \$ \mathrm{~T} 12 \mathrm{sd} \mathrm{d}^{\wedge}$, $\left.\mathrm{d} 1428 \mathrm{c} 1 \$ \mathrm{~T} 24 \mathrm{~m}^{\wedge} 2 / \mathrm{d} 1428 \mathrm{c} 1 \$ \mathrm{~T} 24 \mathrm{sd}^{\wedge} 2\right)$

fit28<-c (d1428t1\$T0m^2/d1428t1\$T0sd^2, d1428t1\$T105m^2/d1428t1\$T105sd^2, d1428t 1 \$T $3 \mathrm{~m}^{\wedge} 2 / \mathrm{d} 1428 \mathrm{t} 1 \$ \mathrm{~T} 3 \mathrm{sd}^{\wedge} 2, \mathrm{~d} 1428 \mathrm{t} 1 \$ \mathrm{~T} 6 \mathrm{~m}^{\wedge} 2 / \mathrm{d} 1428 \mathrm{t} 1 \$ \mathrm{~T} 6 \mathrm{sd}^{\wedge} 2$,

d1428t $1 \$ T 9 m^{\wedge} 2 / d 1428 \operatorname{ti} 1$ T $9 \mathrm{sd}^{\wedge} 2, \mathrm{~d} 1428 \mathrm{t} 1 \$ \mathrm{~T} 12 \mathrm{~m}^{\wedge} 2 / \mathrm{d} 1428 \mathrm{t} 1 \$ \mathrm{~T} 12 \mathrm{sd} 2$,

$\mathrm{d} 1428 \mathrm{t} 1 \$ \mathrm{~T} 24 \mathrm{~m}^{\wedge} 2 / \mathrm{d} 1428 \mathrm{t} 1 \$ \mathrm{~T} 24 \mathrm{sd}^{\wedge} 2$ )

\# plot: histogram of emperical estimates of shape parameter in log

\# scale:

hist $(\log (\mathrm{fic} 28), \mathrm{xlim}=\mathrm{c}(-5,10), \mathrm{n}=50)$

hist $(\log (f i t 28), x \lim =c(-5,10), n=60)$

hist $(\log (f i c 28)$, main="Histogram of Log Transformed empirical estimates of shape parameter",

$\mathrm{xlab}=$ "shape parameter in log scale")

alfac<-mean $(\log (f i c 28))$

betac<-sd $(\log (f i c 28))$

alfat<-mean $(\log ($ fit28) $)$ 


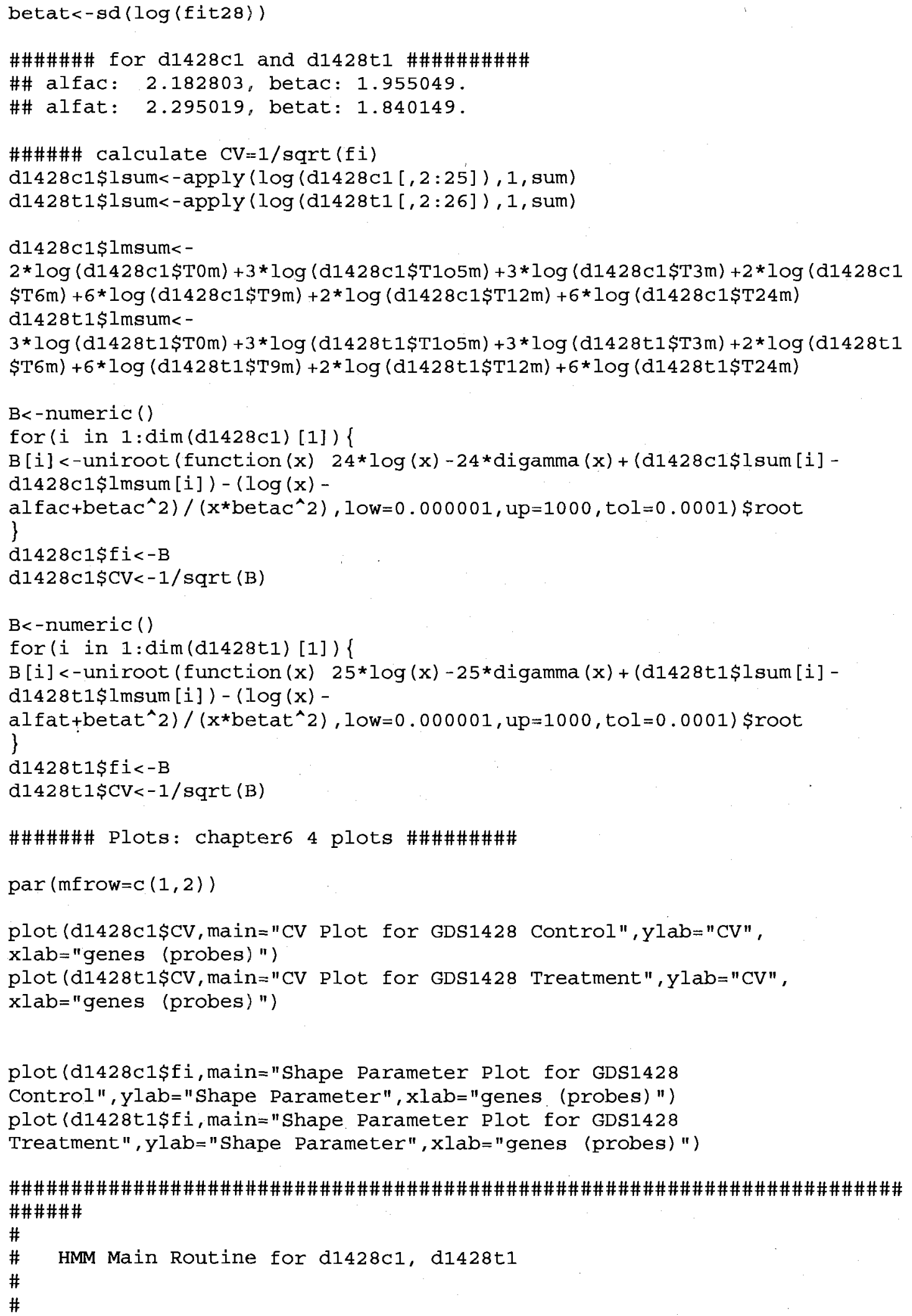




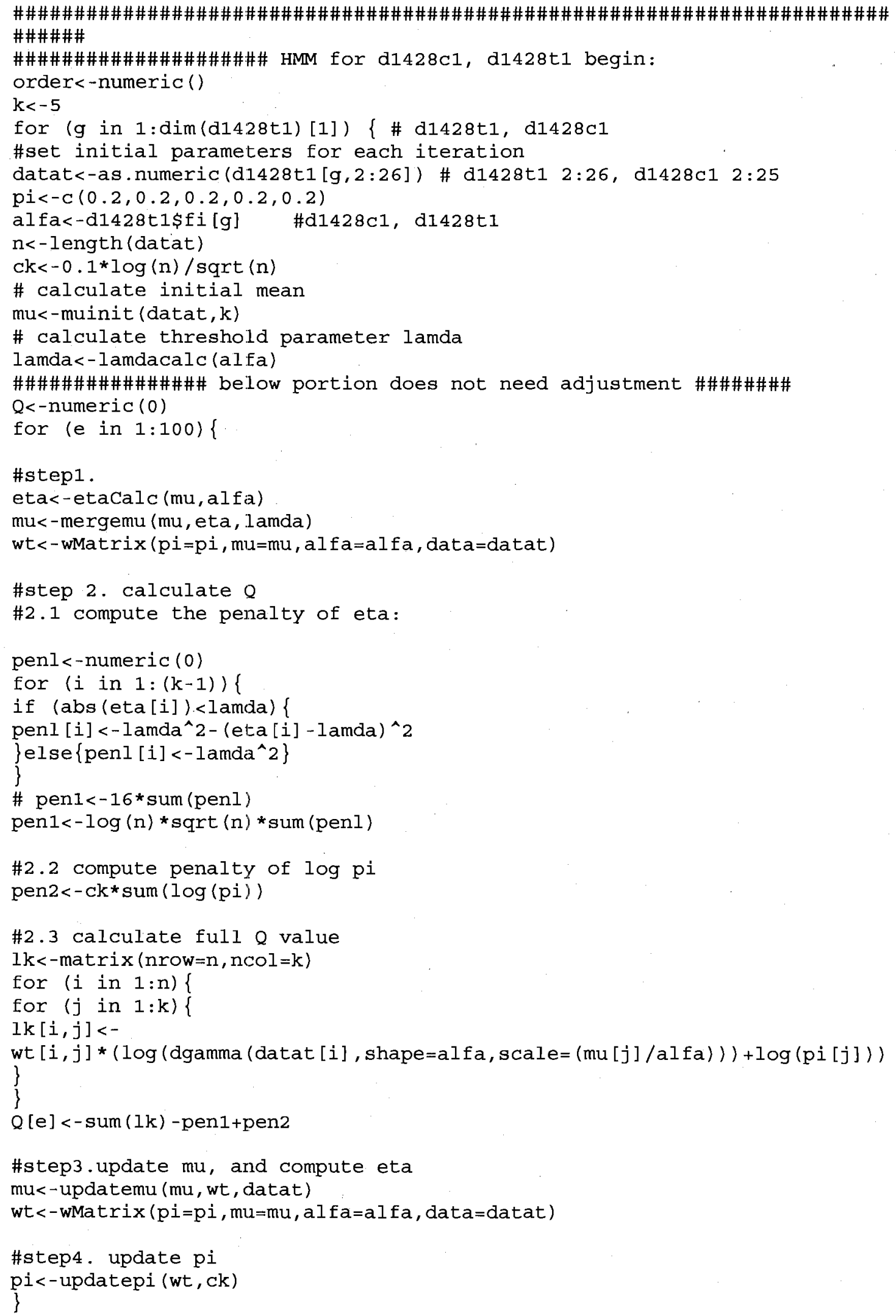




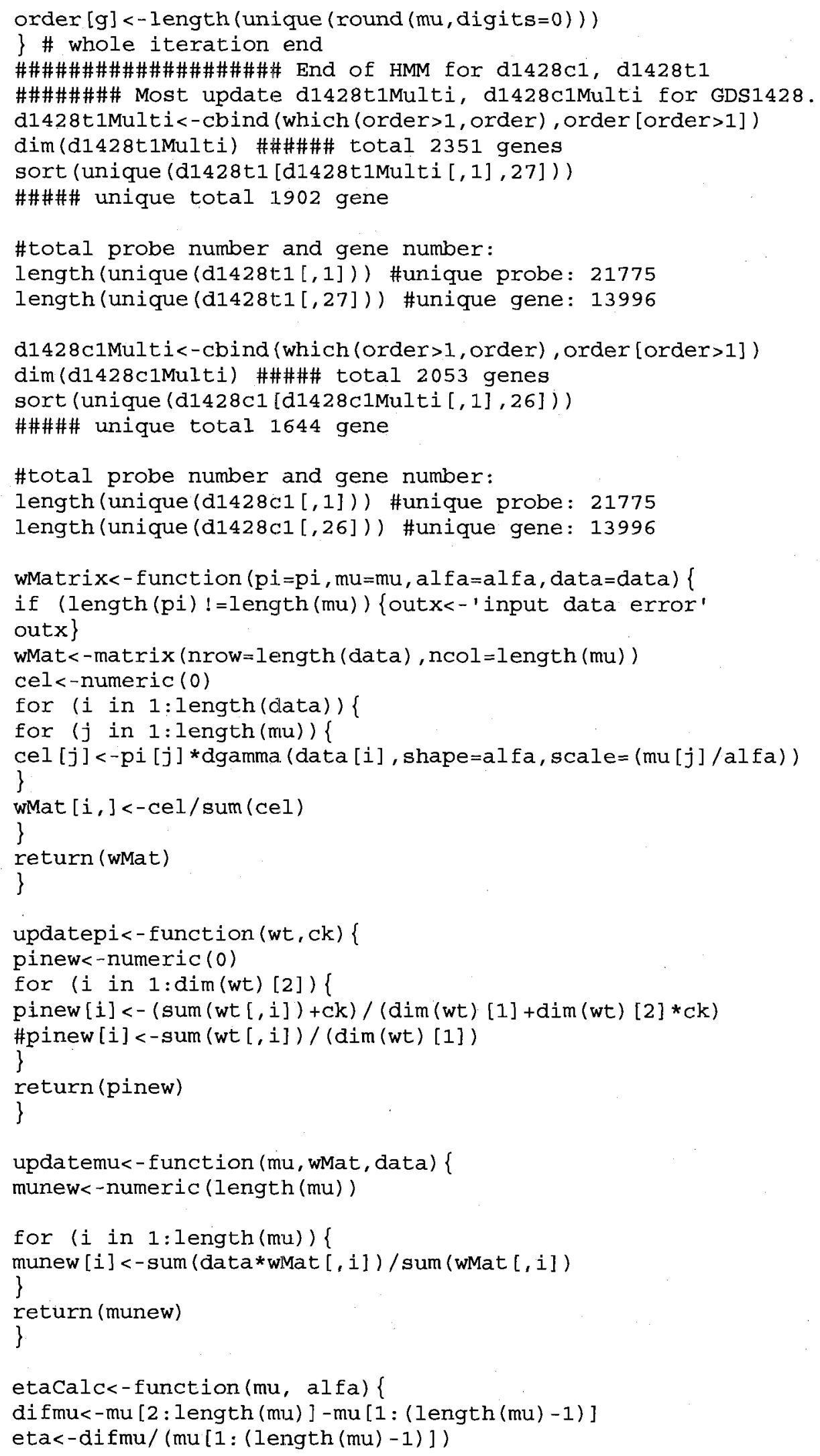




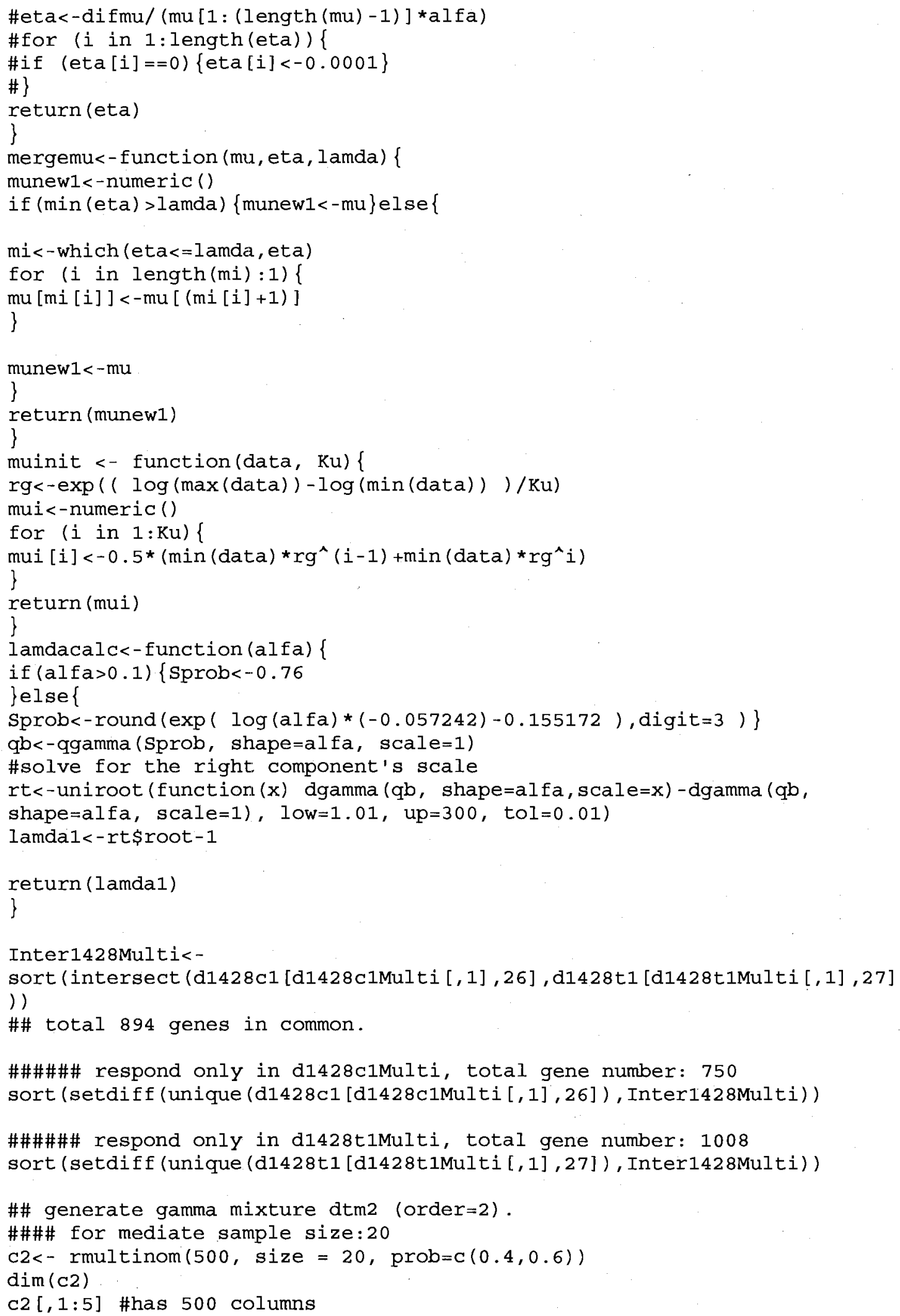




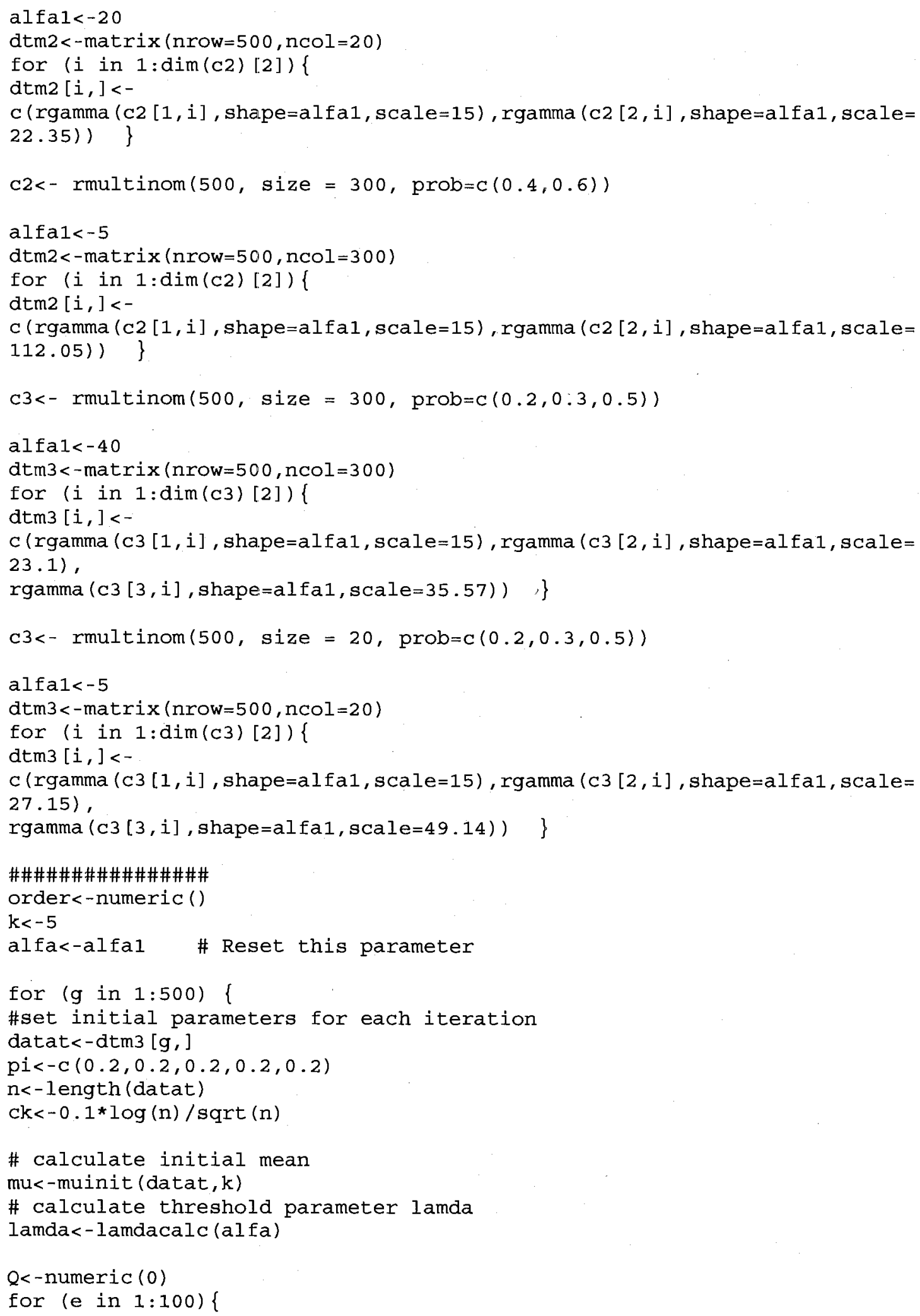




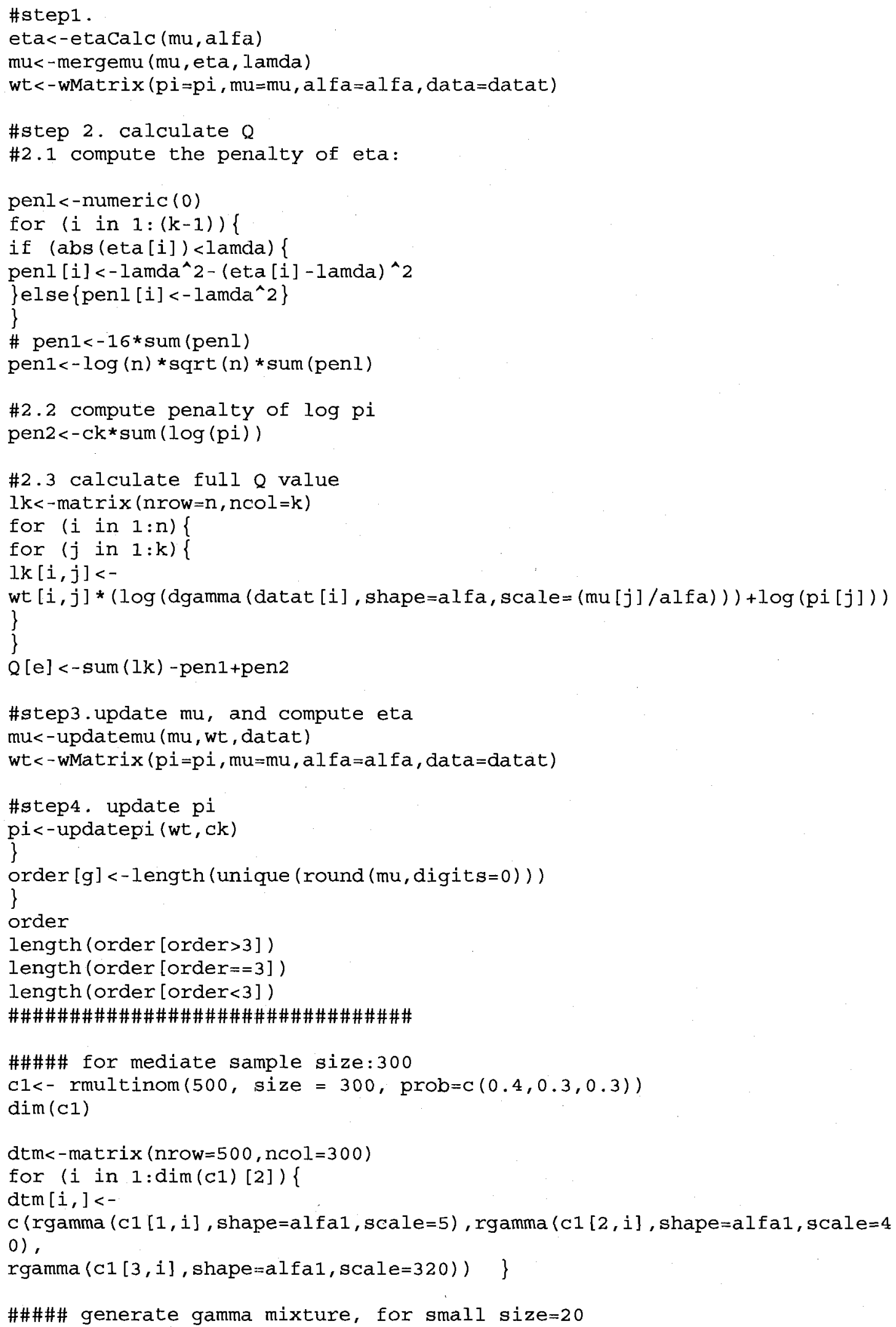




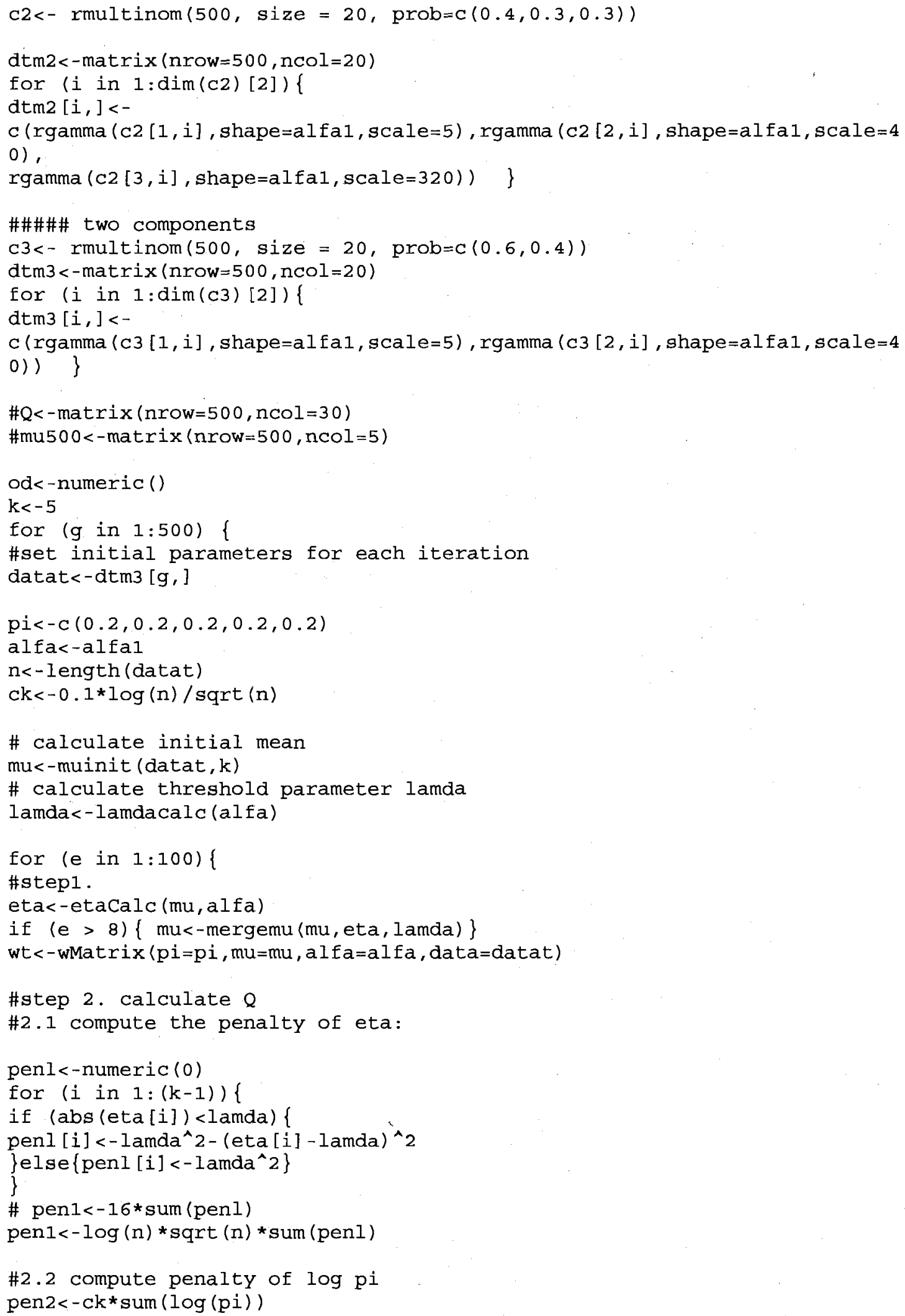




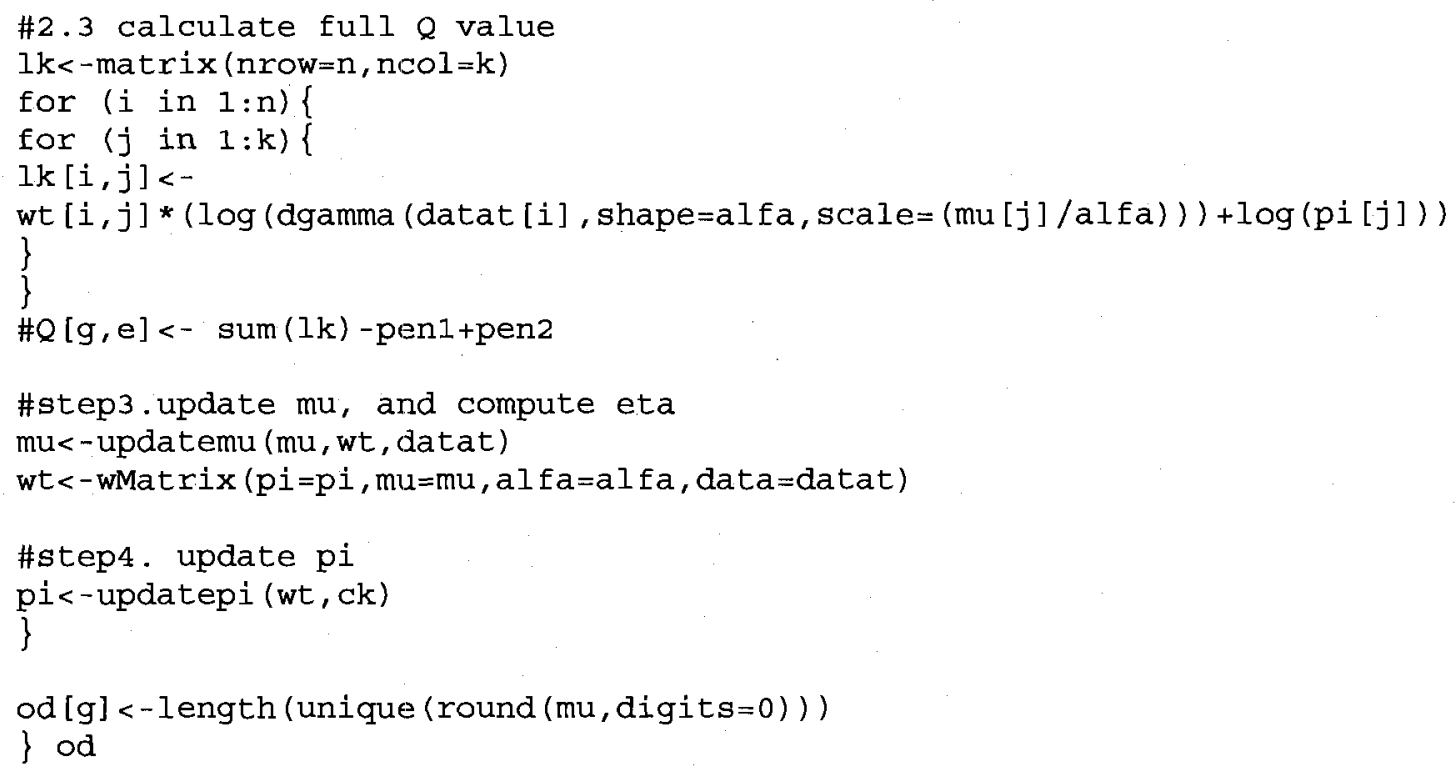

